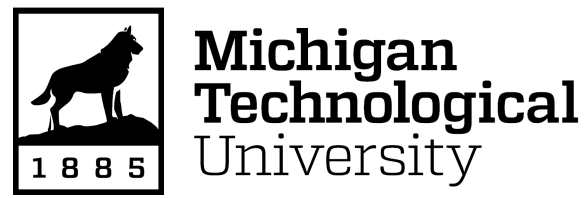

Michigan Technological University Digital Commons @ Michigan Tech

Dissertations, Master's Theses and Master's Reports

2017

\title{
SURFACE ENABLED LAB-ON-A-CHIP (LOC) DEVICE FOR PROTEIN DETECTION AND SEPARATION
}

Zhichao Wang

Michigan Technological University, zhichaow@mtu.edu

Copyright 2017 Zhichao Wang

\section{Recommended Citation}

Wang, Zhichao, "SURFACE ENABLED LAB-ON-A-CHIP (LOC) DEVICE FOR PROTEIN DETECTION AND SEPARATION", Open Access Dissertation, Michigan Technological University, 2017.

https://doi.org/10.37099/mtu.dc.etdr/472

Follow this and additional works at: https://digitalcommons.mtu.edu/etdr

Part of the Biochemical and Biomolecular Engineering Commons, Biomedical Devices and Instrumentation Commons, and the Complex Fluids Commons 


\title{
SURFACE ENABLED LAB-ON-A-CHIP (LOC) DEVICE FOR PROTEIN DETECTION
} AND SEPARATION

\author{
By
}

Zhichao Wang

\begin{abstract}
A DISSERTATION
Submitted in partial fulfillment of the requirements for the degree of DOCTOR OF PHILOSOPHY

In Chemical Engineering
\end{abstract}

MICHIGAN TECHNOLOGICAL UNIVERSITY

2017

(C2017 Zhichao Wang 
This dissertation has been approved in partial fulfillment of the requirements for the Degree of DOCTOR OF PHILOSOPHY in Chemical Engineering.

Department of Chemical Engineering

Dissertation Advisor: $\quad$ Adrienne R. Minerick

Committee Member: Caryn L. Heldt

Committee Member: Lei Pan

Committee Member: Feng Zhao

Department Chair: Pradeep K. Agrawal 


\section{Table of Contents}

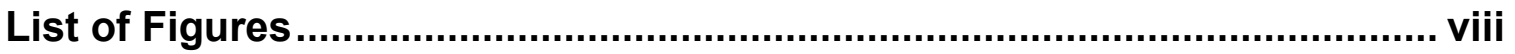

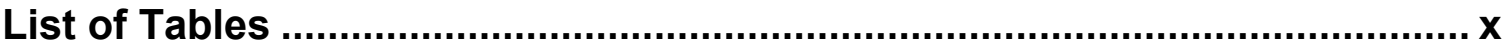

Preface

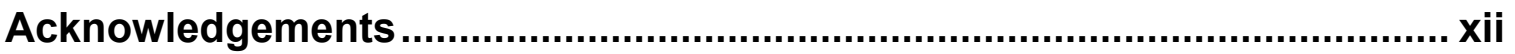

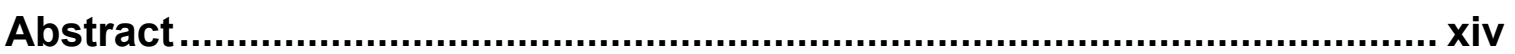

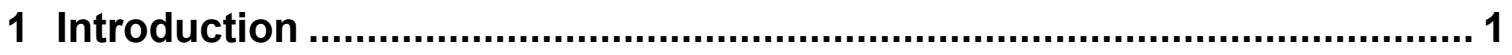

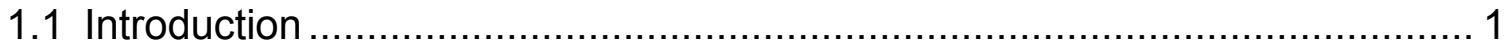

1.2 Current Widespread Protein Analysis Techniques..................................... 2

1.2.1 From DNA to Proteins ....................................................... 2

1.2.2 Protein Purification ................................................................... 5

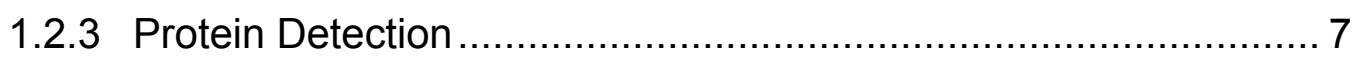

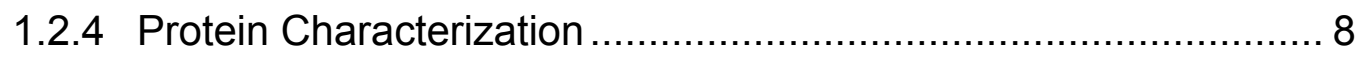

1.2.5 Opportunities in Protein Analysis ........................................... 10

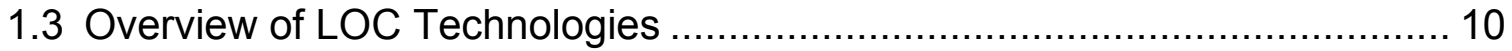

1.4 Lab-on-a-chip Applications on Protein Analysis ......................................... 11

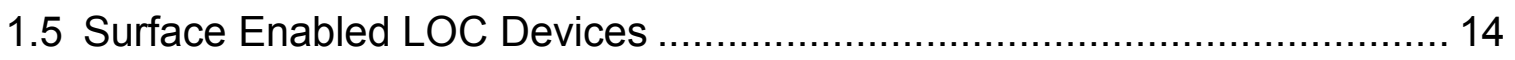

1.5.1 Electrowetting-on-Dielectrics Technique ................................ 15

1.5.2 Surface Enabled Fabrication ................................................ 16

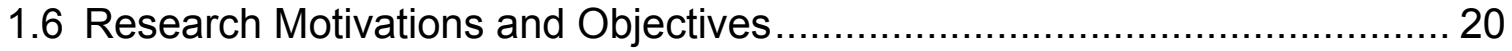

2 A Review of Isoelectric Focusing Techniques ......................................... 23

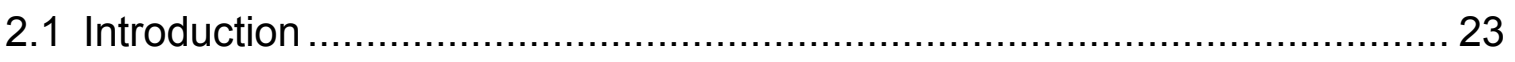

2.2 Theories and Mechanisms of Conventional IEF ...................................... 24

2.3 Theory and Quantification of IEF Efficiency .......................................... 25

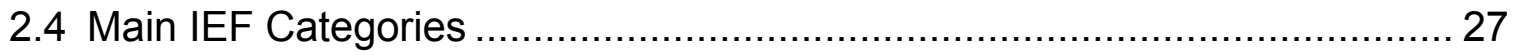

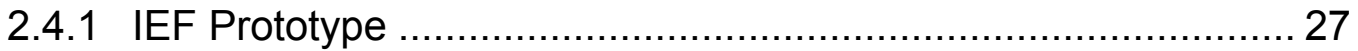

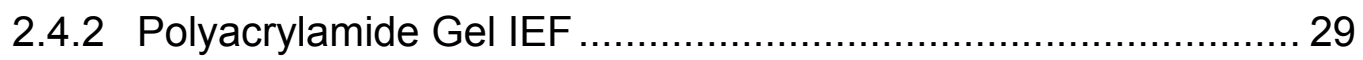

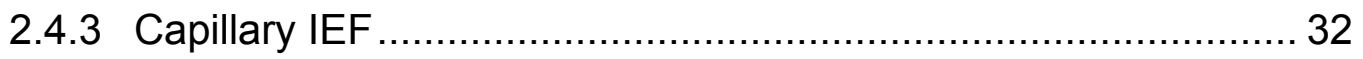

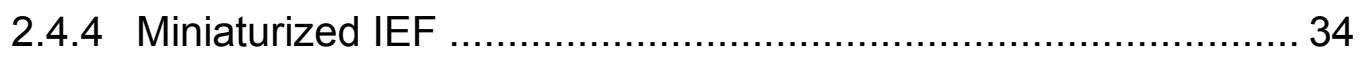

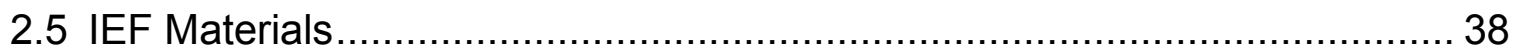


2.5.1 Materials for $\mathrm{pH}$ Gradient Generation...................................... 38

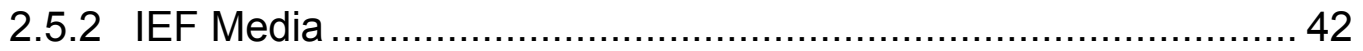

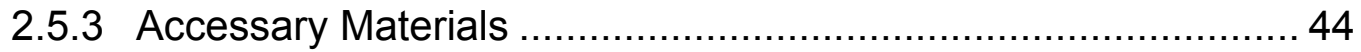

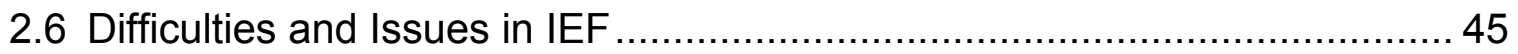

2.6.1 Focusing Time and Applied Voltage ...................................... 45

2.6.2 Unstable pH Gradient ......................................................... 46

2.7 Current Trends and Future Directions of IEF Technology.......................... 48

2.7.1 Current Trends and Future Directions .................................... 48

2.7.2 Surface IEF and Its Application for Protein Separation .............. 48

2.7.3 Quick Outlook of Future IEF ............................................ 51

3 Surface Printing Technique for LOC-IEF Device Fabrication ................... 52

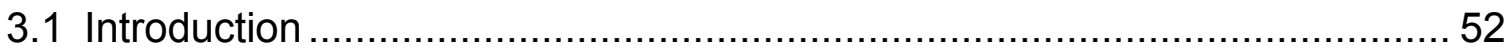

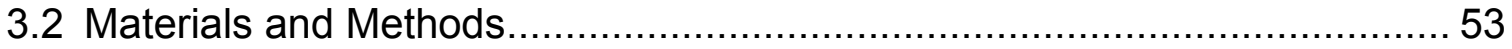

3.2.1 Materials and Instrument .................................................... 53

3.2.2 sIEF Electrodes Microfabrication ............................................ 54

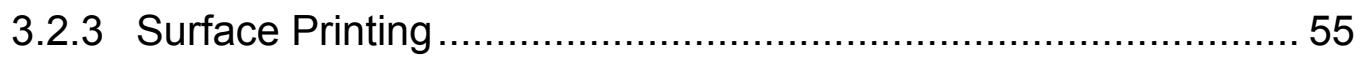

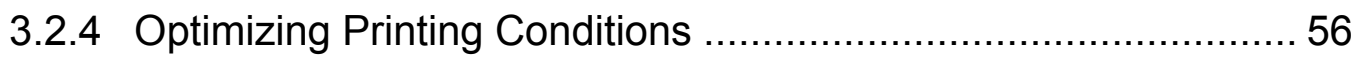

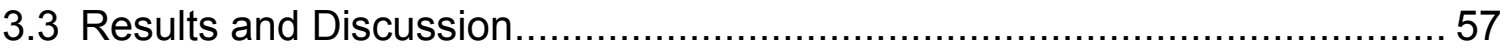

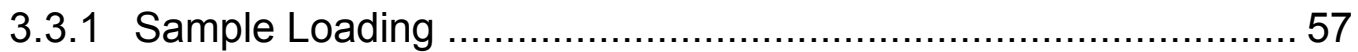

3.3.2 Hydrophilicity/Hydrophobicity of the Surface ........................... 59

3.3.3 Relative Humidity in the Printing Environment.......................... 61

3.3.4 Catalyst Concentration and Related Acrylamide Gelation Time. 63

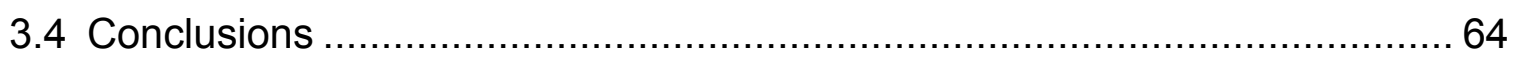

4 Isoelectric Focusing (sIEF) with Carrier Ampholyte pH Gradient ............ 65

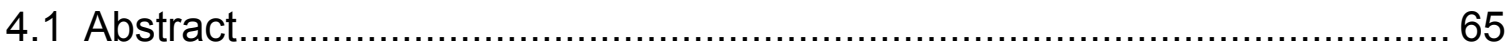

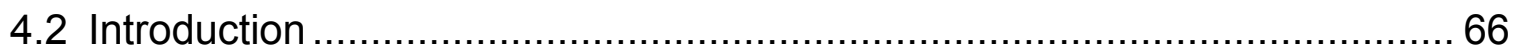

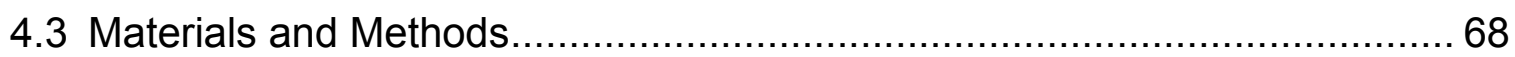

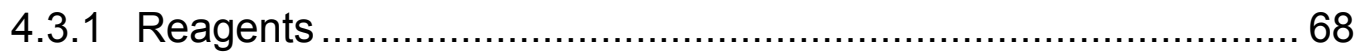

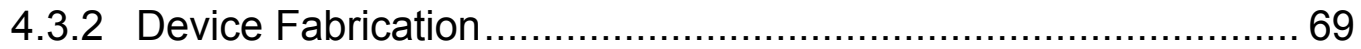

4.3.3 pH Fluorescence Calibration and $\mathrm{pH}$ Gradient Quantification .... 71

4.3.4 Isoelectric Focusing of Protein Mixture and Image Analysis....... 72

4.3.5 sIEF Dependencies and Optimization ................................... 74 
4.3.6 Quantitative sIEF Resolution Power Estimations ...................... 75

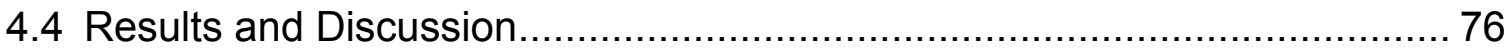

4.4.1 pH Calibration and pH Gradient Imaging ................................ 76

4.4.2 Demonstration of Protein Focusing in SIEF............................ 78

4.4.3 Electric Field Strength Optimization ...................................... 80

4.4.4 Carrier Ampholyte Concentration Optimization ......................... 83

4.4.5 Additional Improvements for SIEF ....................................... 84

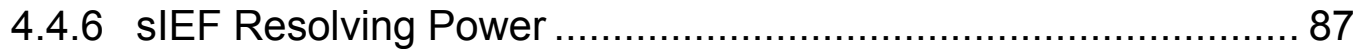

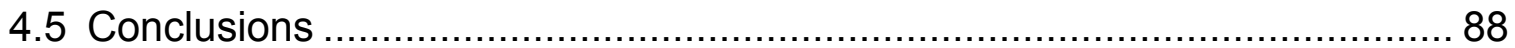

5 Surface Isoelectric Focusing (sIEF) Optimization and Application for Hemoglobin Variants Separation with Narrow Range pH Gradient ............. 90

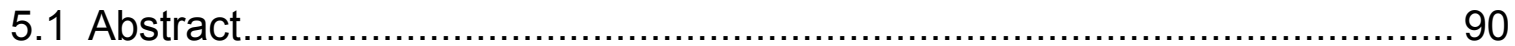

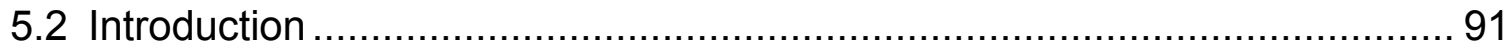

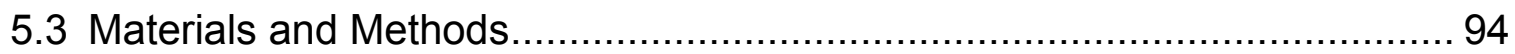

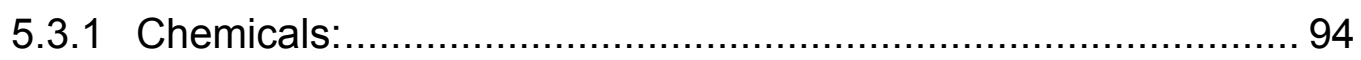

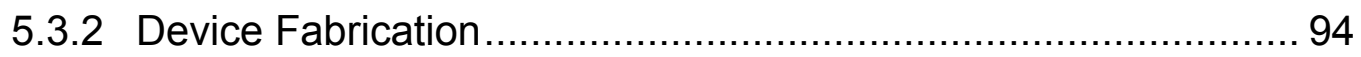

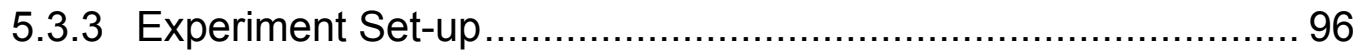

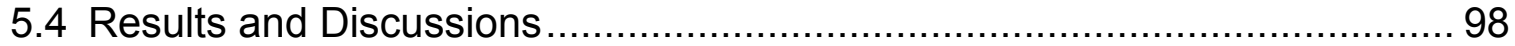

5.4.1 pH Gradient Establishment and Calibration.............................. 98

5.4.2 SIEF Modifications to Improve the PH Gradient: Electrode

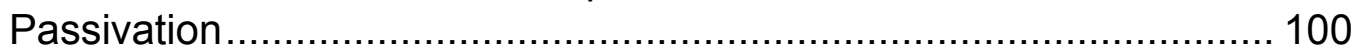

5.4.3 SIEF Modifications: Chemical Additives ................................ 103

5.4.4 Hemoglobin Separation with Narrow Range pH sIEF.............. 104

5.4.5 Pharmalyte ${ }^{\mathrm{TM}}$ and Surfactant Concentration .......................... 107

5.4.6 Peak Capacity Comparison ................................................ 109

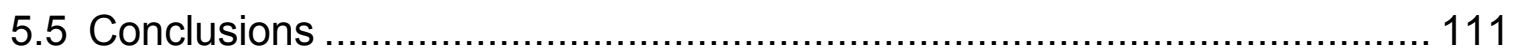

6 Surface Isoelectric Focusing (sIEF) as Auxiliary Tool for Rapid

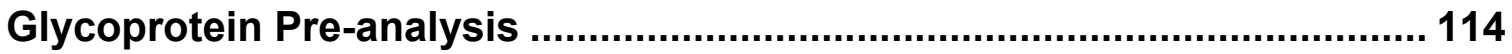

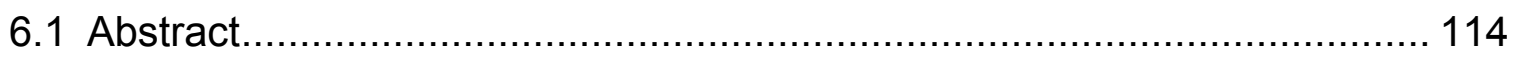

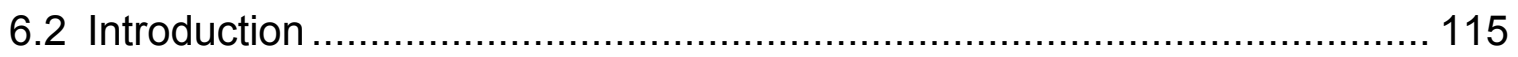

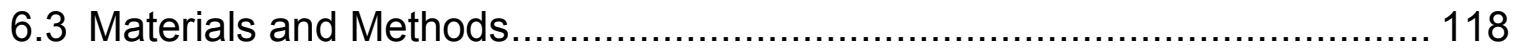

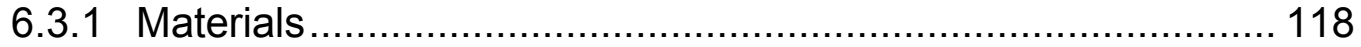




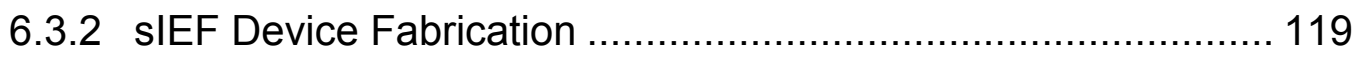

6.3.3 Sample Preparation.......................................................... 120

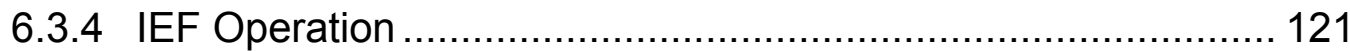

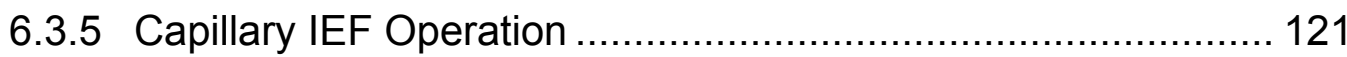

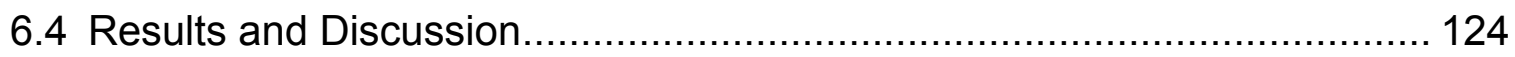

6.4.1 Mouse IgG Separation via SIEF ...................................... 124

6.4.2 Discern Protein Denaturation and Reduction Environment ...... 126

6.4.3 Protein Isoelectric Focusing under Mixed Denaturing and Reducing Conditions ................................................................ 130

6.4.4 sIEF Focusing Verification with CIEF .................................. 131

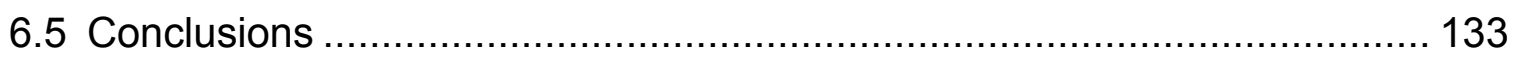

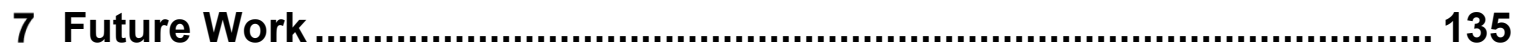

7.1 Quantifying Electroosmotic Flow (EOF) on Hafnium Oxide Surfaces for

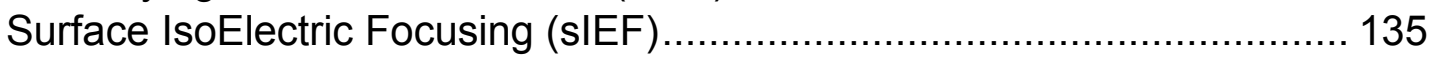

7.2 Further Exploration of the Device Design. ............................................. 136

7.3 Image Detection for Band Identification ............................................. 137

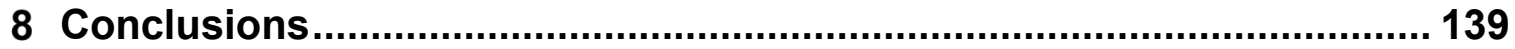

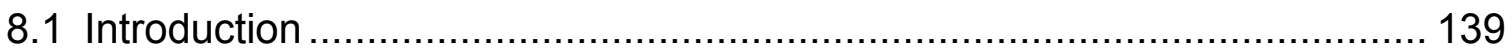

8.2 SIEF Device Design and Fabrication ................................................. 140

8.3 SIEF with Broad Range pH Gradient and Protein Resolving Test ............ 140

8.4 SIEF with Narrow Range pH Gradient and Protein Resolving Test .......... 141

8.5 Glycoprotein Modification Monitoring by sIEF with Narrow Range pH ....... 143

Appendix A: Microfabrication Demonstration and Standard Operating

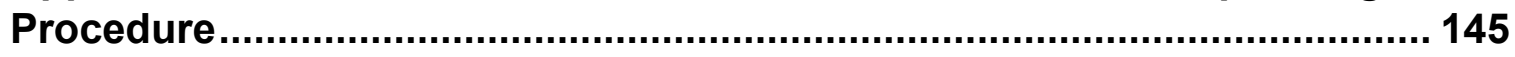

A. Photolithography Masking and Feature Development............................... 148

B. RIE/ICP Etch System for Slide Pre-Deposit Cleaning ................................ 150

C. Electrode Metal Layer Deposition and Liftoff.......................................... 150

D. Passivation Layer: $50 \mathrm{~nm}$ of Sputter Deposited $\mathrm{HfO}_{2}$ Film with 8" Hafnium

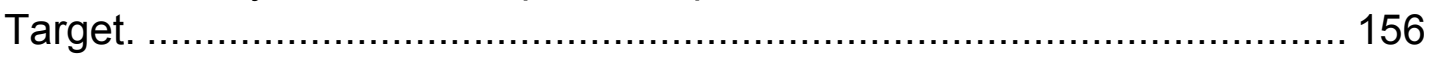

Appendix B: Permission Letters of Reprinted Figures ........................... 159

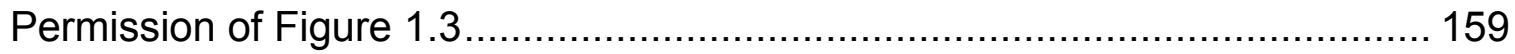

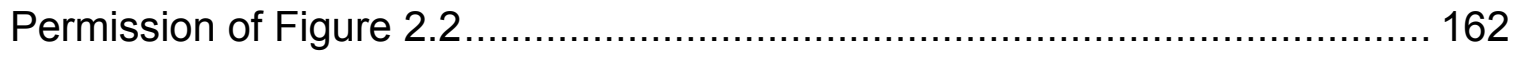

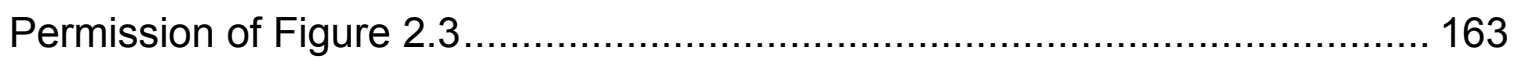




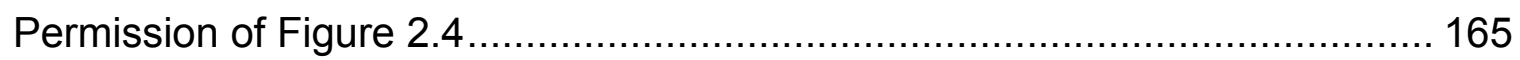

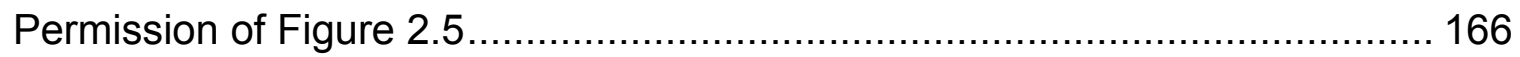

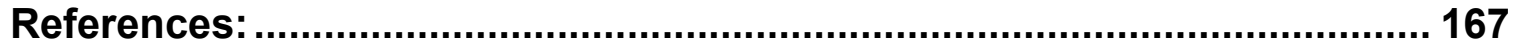




\section{List of Figures}

Figure 1.1 $\mathrm{A}$ formation chain from genetic code to functional proteins ............... 4

Figure 1.2 Protein study flow chart: from single protein species extraction to protein structural characterization on a molecular level.

Figure 1.3 Typical EWOD electrodes set-up............................................... 15

Figure 1.4 BioForce Nano eNabler ${ }^{\mathrm{TM}}$ demonstration ..................................... 18

Figure 2.1 A demonstration of the ampholyte type $\mathrm{pH}$ gradient formatting

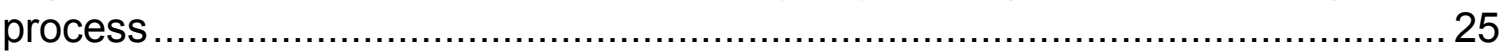

Figure 2.2 Device demonstration of the earliest IEF prototype …..................... 30

Figure 2.3 Demonstration of slab acrylamide gel IEF ................................... 31

Figure 2.4 First $\mu \mathrm{IEF}$ chip structure and the experiment sequence set-up......... 35

Figure 2.5 Device photo and geography dimension of PDMS channel $\mu \mathrm{EF}$

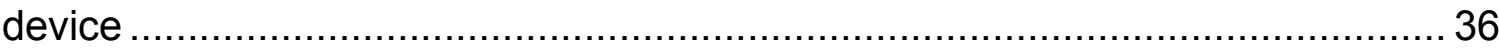

Figure 3.1 The SPT structure (left) and microscope image of SPT tip (right) ....54

Figure 3.2 AutoCAD design of sIEF drive electrode pattern ........................... 55

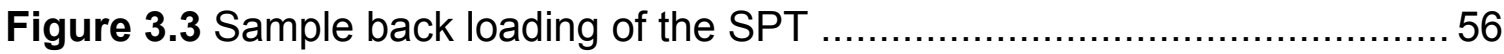

Figure 3.4 SPT tip with varying degrees of contact with the surface................. 58

Figure 3.5 Demonstration of acrylamide gel line drew via front loading of SPT and automated linear vector movement of the SPT.

Figure 3.6 Microscope images of surface printed line and surface contact angle

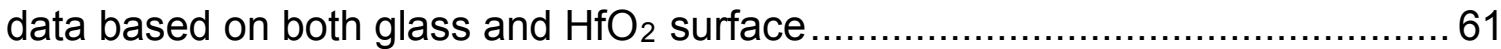

Figure 3.7 Humidity control during printing process...................................... 63

Figure 3.8 Acrylamide gelation photos with different APS concentration........... 64

Figure 4.1 Schematic showing configuration and operation of the surfaceenabled IEF (sIEF) device

Figure 4.2 Images of dynamic pH gradient establishment within the printed polyacrylamide sIEF gels

Figure 4.3 Images of dynamic GFP/R-PE protein mixture focusing within the printed polyacrylamide sIEF gels 
Figure 4.4 Calibration of $\mathrm{pH}$ fluorophores within the polyacrylamide gels......... 77

Figure $4.5 \mathrm{pH}$ gradient establishment and protein focusing in SIEF ................. 79

Figure 4.6 Electric field comparisons of GFP and R-PE protein separations in sIEF gels after 10 minutes in $300 \mathrm{~V} / \mathrm{cm}, 200 \mathrm{~V} / \mathrm{cm}$, and $100 \mathrm{~V} / \mathrm{cm} \ldots \ldots \ldots \ldots \ldots \ldots . . . . . . . .82$

Figure 4.7 Pharmalyte ${ }^{\mathrm{TM}}$ concentration dependence on sIEF gel-based GFP and

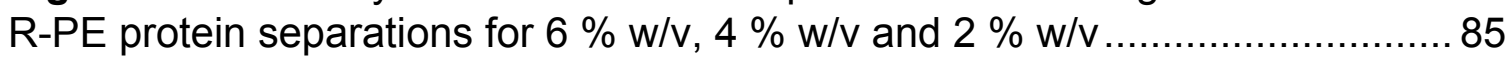

Figure 4.8 Demonstration of surfactant improved focusing efficiency ............... 86

Figure 5.1 Configuration of the electrode-passivated surface-enabled IEF (sIEF) device 96

Figure 5.2 Demonstration of image processing and analysis procedures......... 97

Figure 5.3 Demonstration of image processing and analysis procedures....... 100

Figure 5.4 Microscope images and corresponding intensity profiles of SIEF-

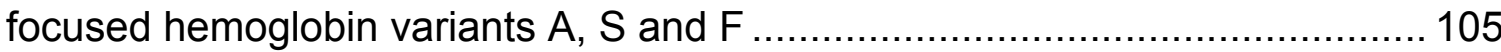

Figure 5.5 Pharmalyte ${ }^{\mathrm{TM}}$ concentration dependency on $\mathrm{HfO}_{2}$ coated device .. 107

Figure 5.6 Surfactant concentration investigate on $\mathrm{HfO}_{2}$ coated device.......... 108

Figure 6.1 Demonstration of the electrode-Hafnium oxide passivated surface-

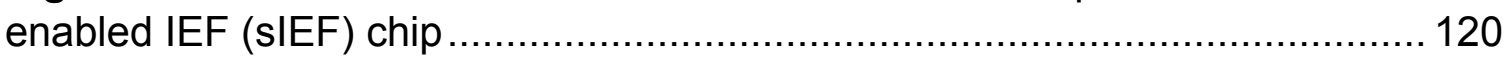

Figure 6.2 Mouse IgG sIEF separation and corresponding image intensity profile. Isoelectric focusing process was with $2 \% \mathrm{w} / \mathrm{v}$ Pharmalyte $^{\mathrm{TM}}(\mathrm{pH}$ 6.7-7.7) and 200

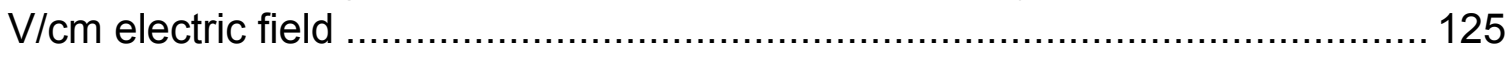

Figure $6.3 \mathrm{pH}$ gradient fits under different environment controls ................... 127

Figure 6.4 Denature and reduce environment control of mouse $\operatorname{lgG}$.............. 129

Figure 6.5 Denature condition control of mouse IgG reduction......................131

Figure 6.6 clEF run results of IgG in different conditions ............................. 133

Figure 7.1 EOF test device design with $\mathrm{HfO}_{2}$ coating................................ 136

Figure 7.2 3-D view of tapered electrode design and a series of parallel sensing electrode lines ca.10 microns across and spaced ca. 10-20 microns apart located between the two sIEF working electrodes 137

Figure 7.3 Interested area image detection demo ...................................... 138 


\section{List of Tables}

Table 1 Utilization of Nano eNabler ${ }^{\mathrm{TM}}$ system in FEMTO fabrication ................. 19

Table $2 \mu$ IEF channel structure, material and pH gradient type ....................... 38

Table 3 Compounds information of commercial CAs .................................... 41

Table 4 Comparison of experimental peak capacity and minimal pl difference from surfactant trial and non-surfactant trial ................................................ 87

Table 5 The $\mathrm{pH}$ fit, Equation 5.1, variables under different sIEF device modifications.

Table 6 Peak capacity calculations of different sIEF passivation and additive conditions

Table 7 Peak capacity comparisons between mainstream techniques

Table 8 clEF run conditions for mAb system.............................................. 123

Table 9 Focused $\lg$ G/lgG fragments locations under different denaturing and reducing conditions 


\section{Preface}

All the contents in Chapter 1, 2, 7, 8, and Appendix A were the original work by the author of this dissertation. The contents in Chapter 3-6 were reorganized based on previously published and intended-to-be-published papers. Details are as described below: Chapter 3 and 4 were based on an academic article that was accepted by Electrophoresis on 19 July, 2017 (DOI: 10.1002/elps.201600565). The author of this dissertation was responsible for collection and analysis of all experimental data. The contents was written by the author of this dissertation under direction of Dr. Adrienne Minerick and our collaborator Dr. Cornelius Ivory from Washington State University. Chapter 5 will be submitted as academic article in Biomicrofluidics. The author of this dissertation was responsible for collection and analysis of all experimental data. The contents was written by the author of this dissertation under direction of Dr. Adrienne Minerick. Chapter 6 will be submitted as academic article (to be determined). The author of this dissertation was responsible for collection and analysis of all surface isoelectric focusing data. The capillary isoelectric focusing experiments were conducted by undergraduate student Logan McMillan from Michigan Technological University and our collaborator $\mathrm{PhD}$ candidate Xavier Redmon from University of Arkansas. The contents was written by the author of this dissertation under direction of Dr. Adrienne Minerick and our collaborator Dr. Christa Hestekin from University of Arkansas. 


\section{Acknowledgements}

If I could pick random day over the course of my work, then it would be really easy for me to imagine my excitement when I got close to completing my degree. However, now that I am writing the last page of my dissertation, my feelings have become complicated. This dissertation indicates not only the work leading me up to my diploma, but also is the first real milestone in my six years living in United States, studying at Michigan Technological University, and working in the Medical micro-Device Engineering Research Laboratory (M.D.-ERL). Throughout these years I have felt at home in this strange country, encountered opportunities to learn things in ways both familiar and unfamiliar to me, and experienced the challenges of cutting edge research. Dozens of individuals have given positivity to my research and life. By writing down this short section at the beginning of my dissertation, I wish to deliver my sincerest acknowledgements to those who have mattered the most during this time.

First and foremost I would like to thank my advisor, Dr. Adrienne R. Minerick. It has been an honor being her Ph.D. student. She has taught me how vigorous a strong work ethic should be, both in research and in life. I appreciate all of her time, ideas, and funding opportunities that helped the completion of my Ph.D. study. Her enthusiasm for her own research motivated me to stay excited in mine as well, even during tough times. Also her joy for life and family provided me with an excellent of how to life and work should be balanced. I am also thankful for the support and suggestions she has made for my future military and industry career paths.

The members of the M.D.-ERL group have contributed hugely to my personal and professional life. This group has offered me the chance to collaborate on ideas and build friendships. I greatly appreciated all of the resources in fabricating the micropatterned electrodes and current measurement of my device offered to me by the Microfabrication Core Facility (MFF) team (Dr. Paul Bergstrom, Dr. Chito 
Kendrick, Mr. William Knudsen, Mr. Michael Chase Mr. Chuck Sannes and Mr. Mark Sloat). Special thanks to Dr. Jaroslaw Drelich, Dr. Craig Friedrich, Dr. Radheshyam Tewari, and Dr. Peter Moran for the resources of the surface wetting test, and surface structure probing. Special thanks to our research collaborators Dr. Cornelius Ivory and Dr. Christa Hestekin, for their time and helpful insights of the sIEF project.

I gratefully acknowledge the funding opportunities that made my Ph.D. program possible. I was given funding support through the U.S. National Science Foundation, and the Teaching Assistant Program in the Chemical Engineering Department at Michigan Technological University.

For this dissertation, I would like to thank my reviewing committee members: Dr. Caryn L. Heldt, Dr. Lei Pan, and Dr. Feng Zhao for their time, interest, insightful questions, and helpful comments.

Last, but certainly not least, I feel incredibly grateful to my family for all their love and encouragement. For my parents, who supported me in all of my career pursuits, and for my girlfriend, who has been supportive and patient during the final stages of my dissertation. I feel so lucky to have all of you! 


\section{Abstract}

Sensitive and selective chemical/biological detection/analysis for proteins is essential for applications such as disease diagnosis, species phenotype identification, product quality control, and sample examination. Lab-on-a-chip (LOC) device provides advantages of fast analysis, reduced amount of sample requirements, and low cost, to magnificently facilitate protein detection research. Isoelectric focusing (IEF) is a strong and reliable electrophoretic technique capable of discerning proteins from complex mixtures based on the isoelectric point $(p /)$ differences. It has experienced plenty of fruitful developments during previous decades which has given it the capability of performing with highly robust and reproducible analysis. This progress has made IEF devices an excellent tool for chemical/biological detection/analysis purposes. In recent years, the trends of simple instrument setting, rapid analysis, small sample requirement, and light labor intensity have inspired the LOC concept to be combined with IEF to evolve it into an "easily-handled chip with hours of analysis" from the earlier method of "working with big and heavy machines in a few days."

Although IEF is already a mature technique being applied, further LOC-IEF developments are still experiencing challenges related to its limitations such as miniaturizing the device scale without harming the resolving/discerning ability. With the facilitation of newly technologically advanced/improved fabrication tools, it is completely possible to address challenges and approach new limits of LOC-IEF. In this dissertation, a surface enabled printing technique, which can transfer liquid to a surface with prescribed patterns, was firstly introduced to IEF device fabrication. By employing surface enabled printing, a surface enabled IEF (sIEF) device running at a scale of 100 times smaller than those previously reported was designed and fabricated. Commercial carrier ampholytes (Pharmalyte ${ }^{\mathrm{TM}}$ ) with different $\mathrm{pH}$ range were engaged to generate a continuous $\mathrm{pH}$ gradient on sIEF device. Device design and optimized fabrication conditions were practically investigated; establishment of $\mathrm{pH}$ gradient was verified by fluorescent dyes; 
dependencies of electric field strength and carrier ampholytes concentration were systematically examined. To further optimize the sIEF system, dependencies of surface treatment and additive chemicals were explored. Fluorescent proteins and peptides were tested for the separation capability of sIEF. Finally, the well optimized sIEF system was used as a tool for real protein (hemoglobin variants and monoclonal antibody isoforms) separations. Hemoglobin variants test results revealed that SIEF is capable of separating amphoteric species with $\mathrm{p} /$ difference as small as $\mathbf{0 . 2}$. Monoclonal protein tests demonstrated the capability of sIEF to be a ready-to-use tool for protein structural change monitoring. In conclusion, this new sIEF approach has lower applied voltages, smaller sample requirements, a relatively quick fabrication process, and reusability, making it more attractive as a portable, user-friendly platform for qualitative protein detection and separation. 


\section{Introduction}

\subsection{Introduction}

Minimized microfluidic device systems, also known as lab-on-a-chip (LOC) or micro total analytical systems ( $\mu \mathrm{TAS}$ ), are small chips with fabricated patterns of miniature fluidic channels and chambers, electrodes, and/or mechanical structures at a micrometer or smaller. Microfluidic devices are an attractive option because they provide advantages of low detection limits, high-resolution, low-cost, lowlabor intensity, and parallel analysis with small sample requirements. LOC has been actively studied for about the last 30 years with applications possibilities in the medical science, biology, environment science, chemical engineering, material science and analytical equipment design fields [6]. Many applications require well designed and delicate LOC devices, whose development is influenced by fabrication techniques and materials properties: rapid prototyping allows for patterns with micro features to be created via computer aided design (CAD) [7, 8], soft lithography offers an opportunity to transfer a micro pattern onto different materials (silicon, glass, metal, polymer, etc), sealing methods can enclose fluid channels making the microdevice dismountable and reusable [9], and improvement of polymer materials can streamline the device fabrication process and decrease the LOC cost [10]. Thus, LOC will continue to evolve alongside emerging microfabrication techniques, which will enable new applications in biological sensing, disease diagnostic testing and other biology related areas.

In this dissertation, research into miniaturized LOC-IEF (surface enabled IEF, sIEF), which is motivated by the requirements of rapid response, robotic device fabrication and small labor expenses is being presented. Initially, the design of the device was initiated based on the physical mechanism of conventional IEF, and then device fabrication was conducted and optimized with multiple considerations, including materials, instrument operation, and environmental conditions. The second part of this dissertation is based on the concluded device fabrication in part one of the sIEF research. Continuous carrier ampholytes type $\mathrm{pH}$ gradient was 
established in the device, and then examined by $\mathrm{pH}$ sensitive fluorescent dye. Fluorescent proteins and peptides were then used as samples for the sIEF device characterization, and possible dependencies based on traditional IEF were studied in this part. The third part of this dissertation involved methodically studying surface modification and the introduction of addictive chemicals, to optimize the whole system for improved resolving ability. Eventually, real complex protein mixtures (hemoglobin variants, monoclonal antibody isoforms) were applied to evaluate the sIEF system.

Experimental results revealed that the sIEF system was capable of handling smaller volumes of proteins, carrier ampholytes, and gels-enabled separations with equivalent resolution to slab gel IEF; therefore, the results of this research is the first time bring IEF techniques down to the nanoliter scale for sample volumes, which is an essential addition for the development of IEF. Also, the small sample requirement and rapid analysis properties of SIEF may contribute to applications in diagnostic/treatment management of proteomic analyses and proteopathies diseases.

In the following contents of this chapter, an introduction of LOC device and IEF technology general concepts, development of LOC-IEF devices, and surface enabled fabrication will be discussed. The motivation behind this research will also be explained as well. This chapter will serve as the storyline for the entire dissertation.

\subsection{Current Widespread Protein Analysis Techniques}

\subsubsection{From DNA to Proteins}

Protein is one type of the essential biomolecules with an important role in both functional and structural processes of all living things. Structurally, proteins are

complex macromolecules composed of different sequences of amino acids and covalent amide linkages, which are coded from deoxyribonucleic acid (DNA) sequences. To better understand gene sequences that code for proteins, current DNA profiling techniques require $\mathrm{mL}$ to $\mu \mathrm{L}$ scale samples and generate accurate 
and reproducible sequence results that can discern sequence anomalies by comparing them to pre-collected DNA databases [11, 12]. While functional proteins are the direct result of the correct translation of the nucleic acid sequence into the amino acid sequence, the discordance between nucleic acid bases and the 20 amino acids can significantly change protein functions. Also most proteins include strategic folding of polypeptide chains (primary structure) to yield a 3-D structure and compacted folded domains. Thus, it is hard to make a direct prediction from the amino acid sequence to a 3-D structure $[13,14]$.

Between DNA's section of relevant genetic information and the protein's amino acid sequence, there is the intermediate ribonucleic acid (RNA) molecule. The gene's sequence from DNA is encoded into an RNA sequence first, and then the RNA message is translated into an amino acid sequence. Like DNA, RNA is also a 2-D sequence. The formation of the final functional protein follows the order of an amino acid sequence initially assembled with the 2-D sequence as a) the primary structure, b) different regions of 2-D sequence form into secondary structures ( $\alpha$-helices, $\beta$-strands, etc.), c) structural elements of primary and secondary build the compact 3-D tertiary structures, and/or d) the primary and secondary structures further arranged into complicated 3-D quaternary units (see Figure 1.1). The dynamics of these folding patterns are still not fully understood and remain a challenge [15]. Therefore, to interpret an accurate determination of 
protein structures and sequence protein information from DNA results is not insufficient for current research demands [16].

Because proteins are the functional building blocks and assemblers of cell components, thorough and systematic information on protein physical properties is critically important, and tools to directly deduce these properties are essential.

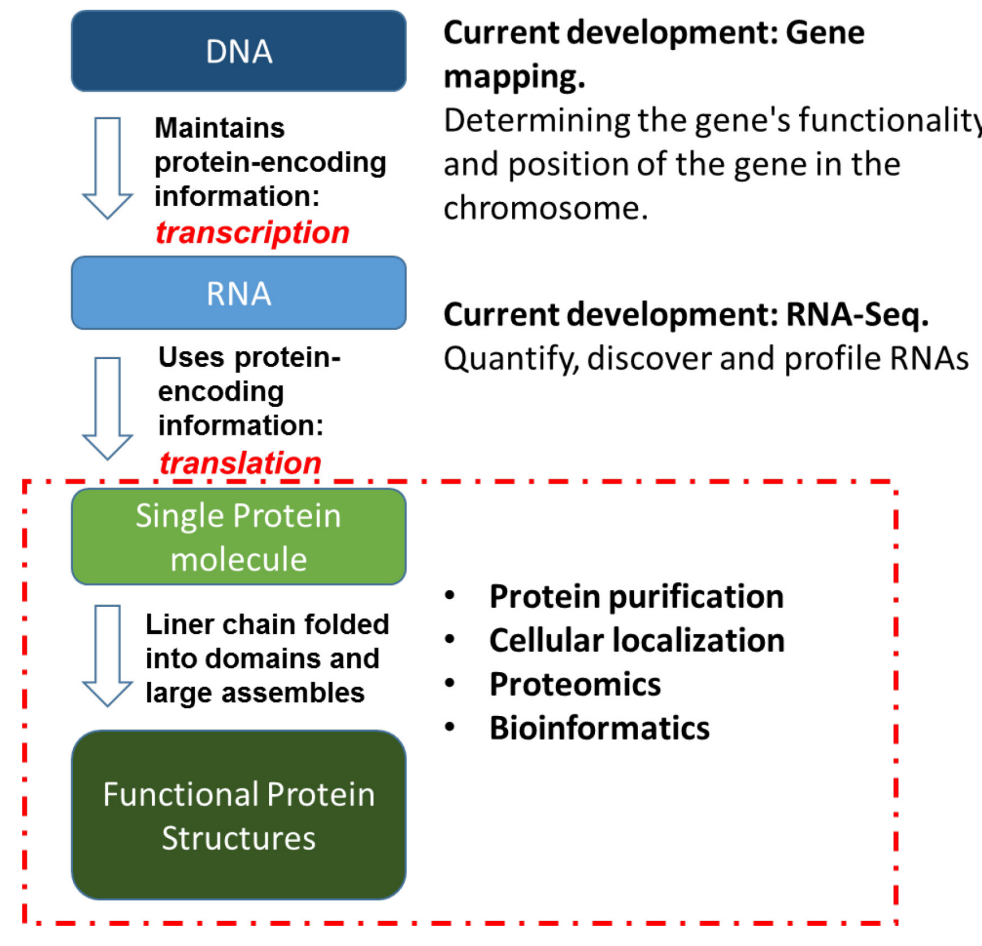

Figure 1.1 A formation chain from genetic code to functional proteins

Researchers also need to directly measure characteristics of dynamic protein upregulation and downregulation in cellular physiology to fully understand healthy and diseased states. This knowledge can lead to improvements in diverse biomedical areas from biosynthesis to pharmaceutical developments to macronutrient physiology studies. 
To systematically investigate protein structure and the mechanisms of action, there is no single methodology capable of running the complete analysis sequence. A reasonable solution is to break protein analysis into several stages and choose different tools for each stage. The whole protein analysis sequence includes protein purification, detection and characterization, as shown in Figure 1.2. For any protein study, purification is necessary to isolate the desired proteins from ca. 10,000 different proteins in a cell. To know the presence of the protein species of interest, tools to detect the molecule are needed. To finally understand protein structures from amino acid composition and sequences, it requires characterization techniques to selectively cleavage and disassemble protein molecules [14, 15]. For each stage, tools such as centrifugation, 2-D electrophoresis, mass spectrometry, etc. are mapped out in Figure 1.2 and will be introduced in the following paragraph.

Methods of Protein Study: Examine

Activities and Structures of Proteins

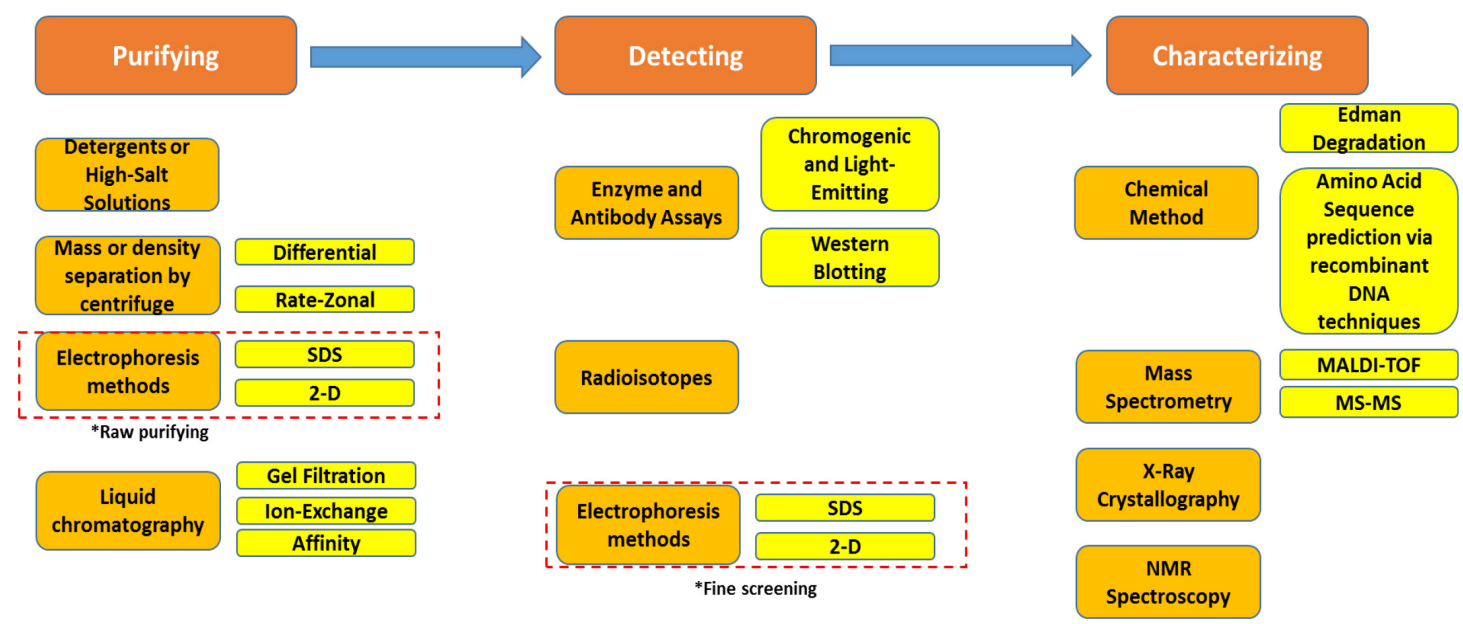

Figure 1.2 Protein study flow chart: from single protein species extraction to protein structural characterization on a molecular level.

\subsubsection{Protein Purification}

Protein studies begin with protein purification, which separates specific proteins from other molecules according to differences in physical properties. In the early 
stage, purification is based on a protein's surface properties (water-solubility, charge), mass, or density. To separate proteins from membranes, surfactants are utilized to disrupt membrane molecular structure by intercalating into phospholipid bilayers and solubilizing lipids and proteins. To separate proteins with different masses or densities, centrifugation with verified rates are used to force sedimentation in prescribed times. These type of protein purification methods can only do rough protein separations with distinctive physical differences [17]. With new instrument developments, novel methods are being used for protein purification to provide superior results; one example is electrophoresis. Electrophoresis is a technique for resolving molecules in a mixture under the influence of an applied electric field. Dissolved molecules in an electric field migrate at a speed determined by their charge to mass ratio. In protein applications, SDS-polyacrylamide gel electrophoresis (PAGE) is the most common and widely used method. Gels are cast between a pair of glass plates by polymerizing acrylamide monomer solutions into polyacrylamide chains and simultaneously cross-linking the chains into a matrix. The pore size of a gel can be varied by adjusting the concentrations of acrylamide and the polymerization catalyst. When protein mixtures are added to a gel and an electric field is energized, different proteins migrate through the gel pores at different rates. Meanwhile, proteins interact with the ionic detergent SDS (sodium dodecylsulfate) before and during gel electrophoresis; as a result, proteins are denatured and forced into extended conformations with similar charge to mass ratios so that mass is the sole determinant of electrophoretic migration [15]. The resolving power of SDS-PAGE is optimal for molecular weights between 1,000 and 300,000 . The variation of gel pore size [18], and the final resolving power can be optimized by adjusting other conditions such as environment $\mathrm{pH}$, buffer solutions, and gel additives [19]. Currently, with the development of gel casting techniques and broader selections of commercial buffer solutions, SDS-PAGE remains one of the most powerful tools for protein purification. 
In addition to SDS-PAGE, liquid chromatography (LC) is also used in protein purification. This method is based on the principle that protein species dissolved in a solution (mobile phase) will bind and dissociate with a solid surface (e.g. packed LC column) with different affinities, which is reflected into different migration rates through the LC packed column. The separation mechanisms vary among LC column types (for example, ion-exchange column for charge based separations [15]). Due to the cost and throughput limits, the use of LC for protein purification is not as common as electrophoresis or physical (detergent wash, centrifugation) methods.

\subsubsection{Protein Detection}

To further analyze the structure and function of proteins, a step for detecting, or assaying specific proteins is important. The earliest way to achieve individual protein detection was the use of specific enzymes and antibody assays. A typical assay capitalizes on a molecule's highly distinctive ability to bind a particular ligand, catalyze a particular reaction, or to be recognized by a specific antibody. To make the results visualized, optical methods such as chromogenic substrates, which change color during the course of the reaction, and fluorescent detection are involved in enzyme and antibody assays. These chromogenic substrates also facilitate the quantitative measurement of enzyme presence. However, due to the properties of selective binding/reaction, every enzymatic/antibody method is constrained by single types of proteins, which prevents enzyme and antibody assays to be a well-adapted general method in protein detections [15, 17].

As an alternative, electrophoresis based methods can also be applied for protein detections. Because some proteins differ in size and shape, yet have nearly identical charge to mass ratios, single dimensional electrophoresis results in little or no separation of molecules of different lengths. In this case, two-dimensional gel electrophoresis (2-DE) is introduced. This most common 2-D combination employs both the charge based (isoelectric point, $\mathrm{p} /$ ) and mass based (molecular weight) separation mechanisms to sort different protein species from complex mixtures. 2-DE is capable of compiling separation information into a protein map 
so that the changes in sample structural expression and post translational modification can be directly tracked. The early stage 2-DE used carrier ampholyte to achieve charge based separation. At that time, due to the immature carrier ampholyte manufacture level, 2-DE had low resolution and reproducibility and did not work well with analytes of very high/low p/ values [20]. With the development of carrier ampholytes, a greater $\mathrm{pH}$ range was possible and the resolving power of 2-DE was improved [21]. The most recent 2-DE applies immobilized $\mathrm{pH}$ gradients (IPG) for protein separations, such that IPG stripes range between $\mathrm{pH} 2.5-12$, thus covering the $\mathrm{p} /$ values of most protein species. Also with the development of computer assisted analyses and 2-D protein databases, the resolving power of 2$\mathrm{DE}$ can reach $\Delta \mathrm{p} /$ of 0.001 , detect less than $1 \mathrm{ng}$ of protein per sample, and up to 5,000 protein species [22]. Another common 2-D combination is gel electrophoresis and antibodies/enzyme assays (Western blotting or immunoblotting). After gel electrophoresis, the pre-separated proteins are transferred or blotted onto a second matrix, then enzymes/antibodies are added to produce a detectable signal. This three-step method (electrotransfer, antibody detection and development) is commonly used to separate proteins and then identify a specific protein of interest [23].

Even faced with competition from other detection tools such as radioisotope labeling [15], 2-DE is still the most common and facile protein detection method due to the advantages of high resolution, commercially standardized operation flow, and simultaneously parallel analysis. However, the well-trained labor requirement, long operation, and analysis times (the whole scenario takes up to a day) makes the tool cost prohibitive and the largest challenge for 2-DE.

\subsubsection{Protein Characterization}

As mentioned earlier, a functional protein is a $3 \mathrm{D}$ complex from amino acid sequence. Types of amino acids, their abundance, their sequence, and their structure are critically important to protein identification. Therefore, characterization tools which can resolve molecular level differences in proteins are in demand. The earliest method to determine the amino acid sequence of a protein 
or smaller polypeptide was Edman degradation. In this procedure the amino group at the $\mathrm{N}$-terminus of a polypeptide was labeled and its amino acid is cleaved from the polypeptide, which left the polypeptide one residue shorter, with a new amino acid at the $\mathrm{N}$-terminus. By repeating this cycle, the polypeptide was systematically shortened, until all the residues were identified [15]. However, this process was slow and the throughput was low. Thus, Edman degradation method was slowly replaced by other more powerful tools.

Mass spectrometry (MS) is now the dominant technique in protein characterization research. The mechanism of MS for proteomics is to ionize protein species and to align the ions based on their mass-to-charge ratio. By recording and analyzing ionized protein molecule pieces, the micro structural information such as covalent structures can be obtained. At the beginning stage, the development of MS on protein analysis was mediocre due to instrument limitations. However, MS rapidly developed in the 1980s, which can be attributed to ionization and mass analyzer techniques. New ionization techniques such as electrospray ionization (ESI) and matrix assisted laser desorption/ionization (MALDI) [24] allowed MS to softly ionize samples to maintain the intact biomolecule pieces during sampling. This enabled MS to access smaller scale fragments at the polypeptide level. Also, new mass analyzers such as time-of-flight (TOF) and charge traps enabled better control of ionized fragments to be delivered to detector, which increased MS resolving power [25]. In the mid-1990s, MS became the mainstream analysis technique for determination of polypeptide molecular mass. Another development of MS for proteomic study was the use of multistage MS instruments. The combinations such as MALDI-MS (or Edman sequencing), TOF-TOF and MS-MS applied one stage to weigh molecules and another stage to analyze information of the selected fragments from previous stage. Through multistage MS, detailed peptides structural features could be inferred from masses analysis of the resulting fragments. Therefore multistage MS instruments were most commonly used to support a range of research strategies in proteomic studies. Recent MS instruments remain the most powerful tools capable of protein identification, 
quantification, and detection of molecular modification, with high throughput and high resolution (up to 12,000 protein/peptides species) [17]. Due to the high purification sample requirements, high cost, long analysis time, and destructive analysis, most applications of proteomic MS in laboratories utilize post-separated samples and do not involve time sensitive protein complex analysis.

\subsubsection{Opportunities in Protein Analysis}

In summary, 2-DE and MS are the mainstream techniques for pre and post analysis of proteins, respectively. However, these tools encounter issues such as long analysis times, complicated sample processing, and larger sample consumption. For these reasons, there exists space for further development of proteomic tools and techniques. Development of a device/instrument able to maintain high resolution and reliability, while simultaneously featuring rapid separation, detection, and identification with smaller samples, would be an impactful advancement for protein analysis.

\subsection{Overview of LOC Technologies}

As described in the introduction, the design and fabrication of an LOC device requires advanced tools and techniques, such as lithography, thin metal layer deposition, and etching. Many of these processes were established with the growth in the semiconductor area $[26,27]$. With the help of microfabrication tools, the scale of LOC can be miniaturized with improved complexity and integrity. LOC's versatility enables a broad range of applications. A common utilization of microdevices in biological/chemical engineering is analyte manipulations; microdevices are employed to mimic laboratory processes in micro/nanoliter volumes by using the microchannels and microchambers fabricated onto the chips [28]. In 1979, Terry and his co-workers miniaturized gas chromatography [29]; sample injection, a capillary column, and a thermal conductivity detector (TCD) were integrated on a 5-cm-diameter silicon wafer with etched gas fluid channel features. Inorganic gas (nitrogen) and several organic gases (hexane, n-pentane, etc.) were successfully detected with retention times reduced from minutes at the macroscale to seconds at the microscale. This research was the earliest 
demonstrated LOC, and it opened the gate to the detection and analysis of macromolecules. Over the last 30 years, LOC devices for cell manipulation [30, 31], blood counting [32], vivo drug delivery [33], protein sensing [34], and even DNA array tests [35], have been developed.

The main direction and motivation for further development of LOC for analyte manipulations include 1) miniaturization of current techniques to make them more versatile and compact (e.g. mini scale gas chromatography [29] and capillary electrophoresis [36-38]); 2) Device portability which could be carried easily and fitted well into the scenario immediately in need where very small sample amounts are available, rapid sample preparation and analysis in seconds or minutes can occur, and online analysis results are required; 3 ) modified chemical/biosensor or detector with enhanced resolution and sensitivity. Eventually, future LOC viability will be determined by user friendliness, test reproducibility, and device robustness.

\subsection{Lab-on-a-chip Applications on Protein Analysis}

Proteins are essential components of living organisms that play vital roles in physiological metabolic pathways. Miscoded or misfolded proteins are the root cause of many diseases including neurodegenerative diseases like Alzheimer's or Parkinson's [39, 40]. Selective protein detection and separation can aid in measuring qualitative and quantitative changes in biological samples for disease diagnosis and management [41]. To reach this goal, techniques that are capable of accurate control and manipulation, rapid analysis, and handling small sample sizes are essential. LOC features practical movement of micro or even nanoscale samples, also it is feasible to be integrated with other analysis tools, such as chromatography, time-of-flight mass spectrometry (TOF-MS) and UV-visible spectroscopy for post sample analysis. These characteristics provide LOC with great potential toward advanced and complicated protein analysis. The earliest prototype of LOC for protein analysis appeared in the early 1990s. Karlsson and his colleagues attached a small microfluidic unit onto a surface plasmon resonance (SPR) sensor to study the kinetics of monoclonal antibody-antigen reactions [42]. The microfluidic unit was used for controllable immobilization of antibody and 
antigen, which led to the observation and calculation of varied affinity and reaction rates. Later on, a micromachining technology was employed in the field of bioinstrumentation. A micron-sized electrical field-flow fractionation ( $\mu$-EFFF) system was introduced by Gale group [43]. Vertical aligned electrodes were patterned by Au and Ti depositing onto a silicon surface, a micro channel (4-6 cm length, 20-30 $\mu \mathrm{m}$ depth and 0.4-8 $\mathrm{mm}$ width) was formed by polyimide and a glass cover. Compared to conventional EFFF, the miniaturized $\mu$-EFFF system has made significant improvements in reducing sample size and separation time. LOC can also be used in the microchip immunoassays field. The relationship between non-specific adsorption of $\lg G$ and the electroosmotic flow ability was investigated by Locascio and his co-workers [44]. A microfluidic channel was formed by imprinting chrome wire onto materials like acrylic, polystyrene and polyester. Non-specific protein `adsorption can be alleviated by reducing electroosmosis (EOF) mobility, which can be achieved by manipulating channel materials. Moreover, LOC techniques can be involved in areas such as isoelectric focusing (IEF) [45], protein biochemical reaction [46], and protein molecule library screening [47].

Generally, LOC protein analysis techniques include physical and chemical methods [41]. Physical methods rely upon spectroscopy-like mechanisms (physical absorption, mass fraction detection, etc); minimized chromatography devices and surface adsorption based protein sensors are common examples [29, 48].The latter example is one of the most popular fields for LOC-protein analysis combination. The motivation of LOC-protein analysis is the small sample analysis capability of proteomics studies. Conventional proteomic analysis was limited in massive information collection, which narrows the access to small molecules. Meanwhile, the automation, cost reduction, and high throughput characteristics were proven to be achieved in chip based devices, which makes LOC a good candidate technologies for the further development of proteomics [49]. There are two main categories among the different LOC systems that have been applied to the proteomic process: microfluidics systems and micro sample arrays. LOC microfluidic devices have evolved for protein analysis where channels less than 1 
$\mathrm{mm}$ guide fluids from sample reservoirs to facilitate analyte separations or guide fluid to waste. Samples include protein or antibody suspensions and buffers [28, 50]. Interesting property changes or phenomenon can be obtained directly from a LOC device (e.g. liquid components separation, fluidic diffusion, $\mathrm{pH}$, velocity, and viscosity). These changes will either be visualized or be correlated to certain parameters (concentration, etc.) of protein species to achieve a detection/analysis purpose. It can be used in protein purification/separation and sample identification in chromatography. For purification/separation, the old school method of IEF for protein separation was successfully integrated onto microfluidic chips. The basic device design includes a microfluidic channel, wire/plane electrodes, and sample injection port/reservoirs [51-54]. Simple sample analysis were demonstrated using these kinds of devices. Furthermore, due to the integrality feature of microfluidic chip, IEF in a microfluidic device was incorporated with chromatography technique such as MS. In Wen's research, inlets for sheath liquid and gas were designed in the microchannel IEF chip. Samples were pre-separated by IEF and then went into the electrospray interface emitter for later MS analysis. This concept unwrapped the path for highly integrated LOC-protein analysis [55]. The second type of micro array based device is a solution for integrated analysis for complicated samples. The basic idea is to create a multiple analytes array, which is easily accessed simultaneously. This array setting will reduce possibilities of sample degrade, nonspecific binding and loss of activity [49]. Techniques included in developing a protein array includes local chemical activation, electrospray deposition, micropads delivered activation, hydrogel stamper and spot synthesis [49, 56-58]. In conclusion, the protein array technique is still undergoing development, and can be facilitated with new techniques, such as electrowetting-on-dielectrics and surface printing $[1,47,59-62]$. There is still a room for the growth of protein array construction.

As previously illustrated, chemical based method is another main technique of LOC protein analysis. Common examples of this method includes dye/stain labeling (organic/metal based, fluorescence/luminescence based, radioactive 
based, etc) and amperometric detection based analysis. A typical use of dye/stain labeling is fluorescent labeled capillary isoelectric focusing (cIEF) for protein separations [63]. In the instrument setting, the capillary usually has an observation window or an optical detector. Compared to conventional cIEF, fluorescent labeled CIEF will keep the spectrum results for analysis, as well as enable the visualization of the focusing process [63, 64]. Amperometric detection based analysis is approached with a three electrodes system which is able to screen the change of generated current that comes from the redox reaction in analytes. The detection will only happen with electroactive analytes, and the detection limit can reach to the femtomolar scale [65]. In LOC-protein analysis applications, potential drop, which is caused by charge accumulation on the electrode surface, will be measured during testing. This mechanism was proven to work for both in vivo and in vitro analysis [66].

In brief, LOC techniques offer a platform that brings protein analysis down to the microscale. In the micro world, deliberate manipulation and accurate analysis are possible to achieve. Also, protein analysis provides LOC an opportunity for real applications that will continuously push its limitations in biomolecule analysis.

\subsection{Surface Enabled LOC Devices}

Traditional LOCs were designed to optimize fluid manipulation inside of device microchannels, which are generated either by hard surface etching of silicon/glass chips or reverse molding of polymer materials from a replicated mask. These types of LOC fabrication methods will certainly have some advantages such like low cost, good reproducibility and controllable device patterning; however, they also face three main challenges: 1) limited adaptability to submicrometer dimensions, which is confined by the resolution of conventional microfabrication techniques, 2) longer analysis times, and 3) operating qualifications of well-trained users. In order to approach the persnickety demands of complex biological and medical applications in modern society, the design and manufacturing of LOC devices, need to make breakthroughs. Opportunely, the rise of novel microfabrication/manufacturing 
techniques have become the driving force that has sparked this LOC device revolution.

\subsubsection{Electrowetting-on-Dielectrics Technique}

One of the best candidates to free LOC devices from their confined microchannels is electrowetting technology. Electrowetting can manipulate tiny fluid droplets on a surface by using externally applied electrical signals under the surface, and thereby control fluid shape and movement [67]. Based on this concept, electrowetting-on-dielectrics (EWOD), which is operated on dielectric/dielectriccoated surfaces, is showing significant potential on LOC fabrication and applications. Figure 1.1 is a typical EWOD device set-up [68]. A droplet positioned between parallel top electrode and bottom electrode arrays can be controlled with alternatingly applied electrical fields, which distort the droplet contact angle and thus their shape. Droplet shape can be restored if the electric field is turned off, but

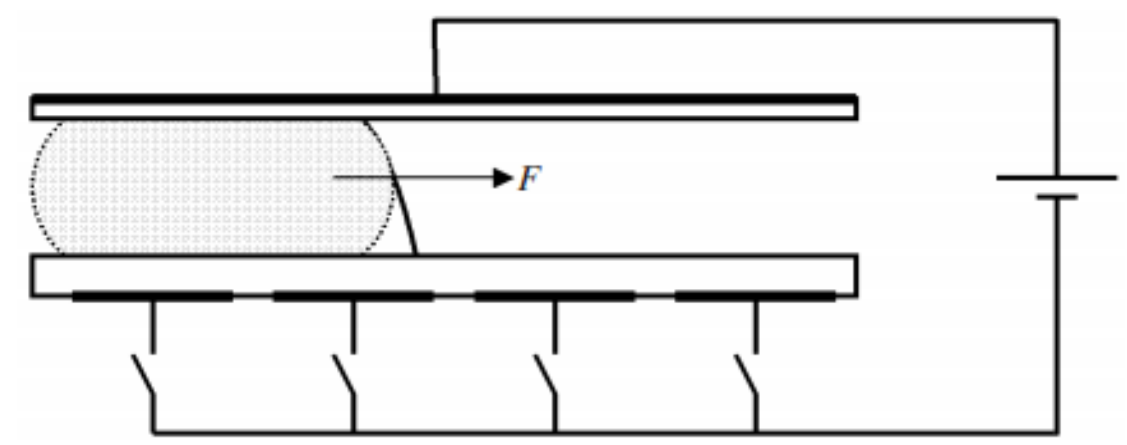

Figure 1.3 Typical EWOD electrodes set-up. Diagram of a typical LOCEWOD system: droplet edges must overlap with at least two adjacent electrodes to get reliable droplet actuation; substrate separations are ca. $100-500 \mu \mathrm{m}$. The order of electrode sizes is of $1 \mathrm{~mm}$, typical droplet volumes are 0.1-1 $\mu \mathrm{L}$ [1]. @ IOP Publishing. Reproduced with permission (see Appendix B). All rights reserved

the position of the drop will depend on the new droplet's edge. This process is capable of moving droplets across a surface.

Characteristics of EWOD are valuable for LOC device operation, especially for the operations that moving, merging, mixing, and splitting droplets at the microliter 
scale. Srinivasan investigated a droplet-based LOC device for clinical diagnostics on human physiological fluids [69]. The EWOD device was composed of a photolithographically patterned metal electrode array on a glass substrate and a continuous ground parallel plane. Fluid was sealed between the upper and lower electrodes and insulated by Parylene $C$ and Teflon 1600 coating. The switching frequency, which is the rate that a droplet can be moved across two adjacent electrodes, was dependent on applied voltage and human body liquid droplets, had different frequency responses. Huh et al. [1] introduced a LOC device with airliquid two phase channels. Two electrodes were underneath the channel, and by applying an electric field, the surface energy of air-liquid surface would change. This led to a flow pattern change used to manipulate flow direction in the channel.

EWOD is an actively developing and exciting technology, which still has some pressing issues effecting LOC device performance [68]: 1) electrowetting requires two electrical contacts to the droplet, which is typically accomplished with sandwiched electrodes, 2) to allow for reliable droplet actuation, droplet edges must overlap with at least two adjacent electrodes such that 100-500 $\mu \mathrm{m}$ electrodes distance requires ca. 0.1-1 $\mu \mathrm{L}$ droplet volumes, and 3) the vapor pressure of water is rather high at room temperature; therefore, evaporation from the small droplets can be a big issue. In summary, EWOD devices successfully bring macro-liquid-manipulation out of confined channels to surfaces and are the first step in new directions being taken for LOC devices.

\subsubsection{Surface Enabled Fabrication}

Surface enabled fabrication is a promising technique first introduced by our group in this document based on fluidics enhanced molecular transfer operations (FEMTO), which utilizes capillary forces with Bioforce Nano eNabler ${ }^{\mathrm{TM}}$ surface patterning tools (SPTs) to transfer a software-programmed liquid pattern onto a surface. This is an attractive technique because it requires fewer reagents via an easy and quick printing process used to create miniaturized or even ultraminiaturized patterns onto arbitrary surfaces. Innovated instruments like BioForce Nano eNabler ${ }^{\mathrm{TM}}$, NanoArrayer ${ }^{\mathrm{TM}}$, etc. have reduced difficulties loading 
nanopipettes or blockage in closed channels, such that it is possible to deliver attoliter to femtoliter volumes of certain solutions/ink to precise locations with welldefined patterns. In addition, surface enabled fabrication will allow a variety of liquid proteins, nucleic acids, lipids colloids, quantum dots, UV-curable adhesives, etchants and catalysts [70] materials to be printed into patterns. In the last 10 years, surface enabled fabrication/FEMTO methods have utilized a quill-type cantileverbased SPT and constructed it for biological molecular patterning [71]. By experimentally generating a Cy3-streptavidin pattern onto a dithiobis-succinimidyl undecanoate (DSU) coated gold surfaces, the reservoir SPT with fluidic transport microchannel and split gap was able to generate biological arrays with 2-3 $\mu \mathrm{m}$ spot size. Later, the same research group [72] reported a construction of multiplexed biomolecular arrays using a modified multiple-cantilever SPT technique to allow for different sample inks to be printed onto a substrate simultaneously.

Numerous liquids have been transferred to arbitrary surfaces including the deposition and patterning of quantum dots [62]. Commercial Streptavidinconjugated quantum dots (QD-SA) were mixed with buffer solution; $200 \mathrm{nl}$ enabled 1-hour of writing. The QD-SA could be written to a gold-coated silicon chip at a fast translation rate of 15-20 $\mathrm{m} \mathrm{s}^{-1}$. Metal nanoparticles can also be patterned by SPT. Onoue and his colleagues [73] successfully fabricated silver nanoparticles metalmask onto glass or silicon oxide wafers. Fountain-pen nanolithography (FPN) of silver ink with the Nano eNabler ${ }^{\mathrm{TM}}$ could be adjusted by optimizing ink and surface conditions. Furthermore, a photomask successfully transferred the pattern to a photoresist film. SPT was also able to pattern $600-n m$ polystyrene (PS) spheres on glass substrates. Subsequent solution evaporation induced self-assembly (EISA) led the PS spheres to form a close-packed hexagonal pattern [61]. The PS sphere diameter could be further reduced by reactive ion etching (RIE), and the 
resulting close packed PS sphere templated sub wavelength periodic structures for surface Plasmon enhanced optical transmission.

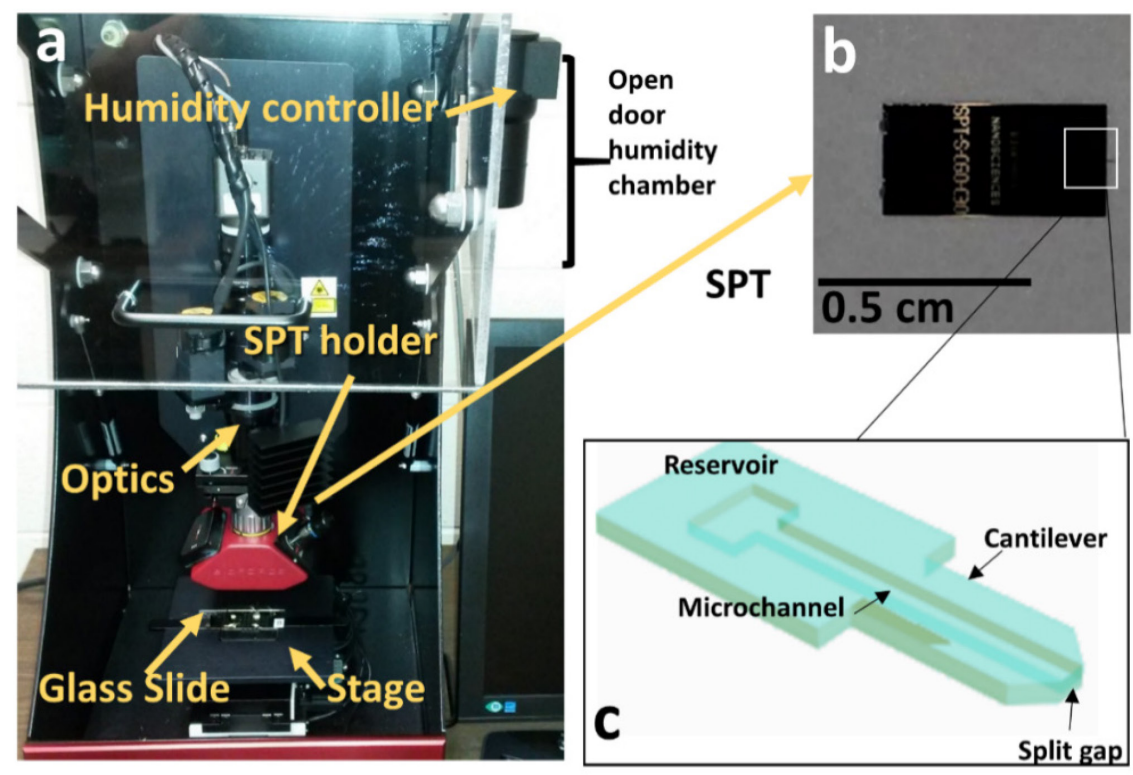

Figure 1.4 BioForce Nano eNabler ${ }^{\mathrm{TM}}$ demonstration. a) The full instrument view. The main parts include the moving stage, optic microscope, surface patterning tool (SPT), SPT holder, and a chamber with controllable humidity. b) Image of SPT. c) SPT structure demonstration

Similar to EWOD, surface enabled fabrication is also compatible with macroscale liquid manipulation. Additional advantages include: 1) sample droplet/fluid patterns being particularly flexible, without an additional pre-design being necessary, 2) it is possible to replace the 3-D sandwiched electrode-sample configuration with a 2-D planar set-up, which could further simplify the whole device fabrication process, and 3) Commercial instruments (BioForce Nano eNabler ${ }^{\mathrm{TM}}$, NanoArrayer ${ }^{\mathrm{TM}}$, can be seen in Figure 1.2) are available. Thus, we predict that fabrication stability and reproducibility can be improved beyond EWOD devices. Surface enabled fabrication is an emerging technique, signified by the few papers (list shown in Table 1) published in this area. We view this as a great opportunity to introduce novel, minimized LOC surface devices. 
Table 1 Utilization of Nano eNabler ${ }^{\mathrm{TM}}$ system in FEMTO fabrication

\begin{tabular}{|c|c|c|}
\hline Author & Ink components & Substrate \\
\hline Baba [74] & $\begin{array}{l}\text { SWNTs, organic } \\
\text { semiconductor }\end{array}$ & Silicon wafer \\
\hline Cady [75] & DNA & $\mathrm{HfO}_{2}$ \\
\hline Fahrenkopf [76] & DNA & $\begin{array}{l}\mathrm{ZrO}_{2}, \mathrm{AlGaN}, \mathrm{GaN}, \text { and } \\
\mathrm{HfO}_{2}\end{array}$ \\
\hline Féréol [77] & Protein & Poly-L-lysine \\
\hline Huang $[77,78]$ & $\begin{array}{l}\text { Extracellular matrix (ECM), } \\
\text { dye }\end{array}$ & Epoxide-modified glass \\
\hline Islam [79] & C-reactive protein (CRP) & $\begin{array}{l}\text { 3-mercaptopropyl } \\
\text { trimethoxysilane-coated }\end{array}$ \\
\hline Korostynska [80] & Polymers & Silicon wafer \\
\hline Luo [61] & Polystyrene nanospheres (PS) & Glass \\
\hline Lynch [81] & Protein & $\begin{array}{l}\text { Gold-coated silicon } \\
\text { wafers }\end{array}$ \\
\hline Mei [82] & ECM protein, dyes & $\begin{array}{l}\text { Octyltrichlorosilane } \\
\text { (OTS) treated glass }\end{array}$ \\
\hline Neto [83] & Biomaterials/proteins & $\begin{array}{l}\text { Superhydrophobic } \\
\text { polystyrene (PS) surfaces }\end{array}$ \\
\hline Onoue [84] & $\begin{array}{l}\text { 3-Aminopropyltriethoxy silane } \\
\text { (APS), 2-(4- } \\
\text { Pyridylethil)triethoxysilane }\end{array}$ & $\begin{array}{l}\text { Silicon and thermal oxide } \\
\text { silicon } \\
\text { wafer }\end{array}$ \\
\hline Onoue [73] & Silver nano particle & $\begin{array}{l}\text { glasses and silicon and } \\
\text { oxide wafers }\end{array}$ \\
\hline Vengasandra [62] & $\begin{array}{l}\text { QDs } \\
\text { conjugated to streptavidin }\end{array}$ & Gold coated silicon chip \\
\hline $\mathrm{Xu}[71,72]$ & Cy3-streptavidin & DSU coated gold \\
\hline
\end{tabular}




\subsection{Research Motivations and Objectives}

Protein detection and separation via a LOC device is a popular research area with rapid growth. One of the most attractive facts about LOC is the capability of it being a miniaturized platform capable of carrying out conventional protein tests such as chromatography, electrophoresis, and sample sensing [85]. For protein detection and separation, isoelectric focusing (IEF) is one of the most effective methods capable of separating and grouping different protein species from a mixture according to their isoelectric points ( $p /$ value) differences. The combination of LOC and IEF is really motivating because it has great potential as a commercialized product that features both high resolution, good reliability inherited from conventional IEF, rapid detection, and less sample requirement descend from LOC [86-89].

The current LOC-IEF devices are at the millimeter scale, it has been reported that IEF pH gradient stability and separation time would be improved with decreasing separation length was decreasinng [90-92]. However, subjected to the resolution limits of conventional fabrication techniques, it is hard to further decrease the scale of IEF. In this dissertation, a novel surface enabled LOC-IEF device concept is presented, with the help of surface printing techniques. This project is aiming to develop an IEF device at the scale of $10^{2} \mu \mathrm{m}$, which is 2 magnitudes smaller than previously existing LOC-IEF devices. This device has characteristics including simple fabrication, low cost, fast operation, and less sample requirements. In the meantime, the resolution of the LOC-IEF device is equivalent to the conventional IEF apparatus. The main story of this work follows the outline below:

1) Establishment of surface enabled isoelectric focusing (sIEF). Our work aims to advance IEF via the surface enabled fabrication to achieve IEF at magnitudes of 100 times smaller than those previously reported [4, 93]. Polyacrylamide gels containing carrier ampholytes (CA) will be used to generate the $\mathrm{pH}$ gradients environment within which proteins can be focused to their $\mathrm{pl}$. Surface enabled fabrication will be utilized to print IEF gels across parallel gold electrodes on a glass chip. The operating 
parameters include gel composition and polymerization time, $\mathrm{pH}$ gradient range, surface treatment, microprinting conditions, and electrode potentials, which will be examined to determine optimized experimental conditions. The finalized device fabrication and operation will be utilized in all IEF working of this dissertation.

2) Examination of $\mathrm{pH}$ gradient in the SIEF device. Performed to qualitatively and quantitatively study the $\mathrm{pH}$ gradient generation along the printed gel lines. Two $\mathrm{pH}$ inspecting systems are applied in this work: $\mathrm{pH}$ sensitive fluorescent dyes and fluorescent peptides $\mathrm{p} /$ markers with known $\mathrm{p} /$ value. The established $\mathrm{pH}$ gradient is monitored under a microscope, and the corresponding image intensity profiles are analyzed. At last, calibration curves showing the $\mathrm{pH}$-location correlation are fitted based on experimental data.

3) IEF performance test using fluorescent proteins. With the proven device concept and $\mathrm{pH}$ gradient, protein mixture IEF experiments are conducted in the sIEF device to test its detection/separation capability. A two-protein system which has fluorescent protein species with large $p /$ value difference is used as sample for tests. Similar to the $\mathrm{pH}$ gradient test, microscope images are recorded during the IEF process and the corresponding image intensity profiles are drawn. Two IEF characteristic parameters: peak capacity and minimum resolvable $\mathrm{p} /$ differences are calculated and compared by species based on the IEF peaks in the intensity spectrum.

4) Device optimization via surface modification and additive chemicals. Similar to conventional IEF, instable $\mathrm{pH}$ gradient phenomenon also observed in SIEF system. To suppress $\mathrm{pH}$ instability and improve the SIEF performance, electrode surface passivation and adding additive chemicals into the gel system are investigated. By passivating electrode surface, Faradaic reaction which can generate extra anions and cations could be controlled; by introducing additive chemicals, $\mathrm{pH}$ mobilizing force- 
electroosmosis flow (EOF) could be reduced, as does the protein-protein reaction and protein-surface reactions.

5) Real proteins test in the optimized sIEF device: IEF of more complicated proteins systems are eventually evaluated in this part of the work. Three hemoglobin variants with less than $0.2 \mathrm{p} /$ difference and glycosylated monoclonal antibody isoforms are separated via sIEF. This series of IEF tests is targeting the separation limits of sIEF platform, as well as exploring the potential in complex biological and medical sample detection and analysis. 


\section{A Review of Isoelectric Focusing Techniques}

\subsection{Introduction}

Isoelectric focusing (IEF) is a powerful electromigration technique for identifying, , separating and analyzing amphoteric molecules such as proteins and peptides, which makes IEF fundamental to medical diagnostics of proteopathy diseases involving protein abnormalities and pharmaceutical screening of therapeutic proteins. Traditionally, IEF is performed using large scale slab gels in clinical labs. The operation is resource, labor, and time intensive, which limits the rapid analysis in clinical/commercial applications. Beginning in the 1990s, more researches have been focused on scaling down IEF, in the aim of short analysis time, easy operation, and high integration, with comparable or even better resolution compared with conventional IEF. The main stream of IEF development is the replacement of large scale IEF by small volume, genomic and affinity approaches. Efforts to miniaturize and improve IEF have included channel-based microdevices with ion-exchange membranes, reservoirs, and reagent-release capillary arrays [88, 94-98], and capillary IEF (cIEF) [68, 99, 100]. Unfortunately, sample recovery from the channels remains cumbersome, and thus limit applications and accessibility to lay users. This demonstrates the demand in scientific and industrial sectors for even smaller-scale IEF technologies, while concurrently showing the need for simpler preparation, simpler operational requirements, versatility, and ease of focused spot recovery for subsequent protein characterizations [101]. As described previously, this dissertation focuses on the minimization method of IEF so the current IEF technique could be further explored. The inspirations and comparisons from microscale IEF will be the key point of this chapter.

In the beginning of this review, however, conventional IEF techniques will still be involved. Theories and mechanisms of IEF will be defined at the beginning, including the working mechanism, dominated physical phenomenon, dependencies, criteria of IEF performance (peak capacity, resolution, etc.). After that, a history of IEF will be introduced by category, and will cover IEF from its very early prototype to recently developed techniques. To establish an IEF system, 
materials will be summarized in the following part to provide a guide of experiment options. The last part will be an overview of future trends, and our surface isoelectric focusing (sIEF) concept will be introduced eventually, which is theoretically reasonable and applicable.

\subsection{Theories and Mechanisms of Conventional IEF}

During the first time that Kolin introduced the IEF prototype in the 1960s, ampholytes were being used for $\mathrm{pH}$ gradient generation [102, 103]. Ampholytebased IEF is the oldest among the electrophoretic equilibrium gradient methods [2, 30-32]. As a mechanism, IEF is conducted with the $\mathrm{pH}$ distribution of carrier ampholyte (CA) molecules. (Commercial available CA including Pharmalyte ${ }^{\mathrm{TM}}$, Bio-Lyte, Ampholine, etc.). When being subjected to an electric field, the negatively and positively charged CAs migrate electrophoretically and cease to the place where the location $\mathrm{pH}$ is equal to their own isoelectric points. Due to the amphoteric feature, each $\mathrm{CA}$ is able to protect the environment $\mathrm{pH}$ from enormous change; therefore, the $\mathrm{pH}$ distribution in IEF separation space is composed of several fragments with $\mathrm{pH}$ equal to the $\mathrm{p} /$ values of local CAs. By adding different $\mathrm{CAs}$ with gradually changed $\mathrm{p} /$ values, a smooth, linear $\mathrm{pH}$ gradient can be established within the IEF separation space (shown in Fig 2.1) [2, 19, 33]. In order to make the established $\mathrm{pH}$ gradient as smooth as possible, commercial CAs are composed of more than 300 compounds with more than 1000 isoforms [21]. Similar to CAs, amphoteric analytes (proteins, peptides, etc) charges according to the surrounding $\mathrm{pH}$ during IEF separation processing: the analyte located in the place that $\mathrm{pH}$ is below its isoelectric point $(p /)$ will be positively charged and migrate toward the cathode; if the environment $\mathrm{pH}$ is above the analytes' $\mathrm{pl}$, then the analyte will be negatively charged and migrate towards the anode [34]. At an analytes' isoelectric focal point, it has zero charge and zero electrophoretic mobility and migration ceases. In the meantime, diffusive or convective migration and electrophoretic force driven flow are the counteracted factors for IEF, which bring unwanted phenomena such as band broadening [32]. However large amphoteric molecules, 
like proteins, have small diffusivities; therefore, they are able to stay focused as tight bands for a certain amount of time before broadening happens.

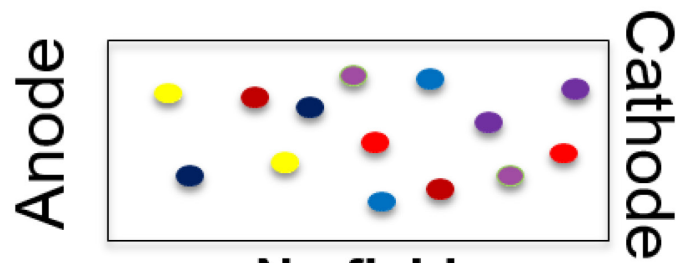

No field

\section{Ampholytic species p/ order} $>0>00>0>0>0$

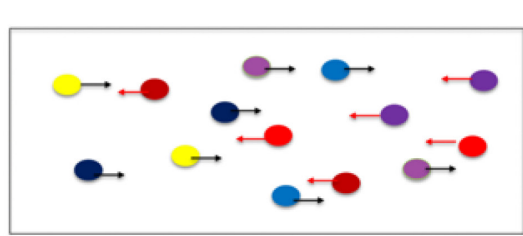

Field Applied

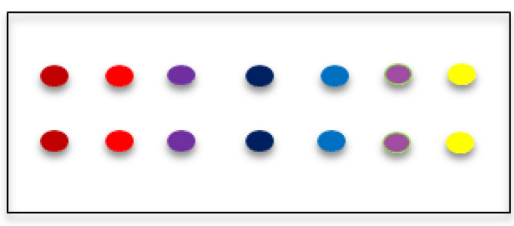

Steady state

Figure 2.1 A demonstration of the ampholyte type $\mathrm{pH}$ gradient formatting process

\subsection{Theory and Quantification of IEF Efficiency}

To evaluate the performance of IEF, some key concepts need to be introduced. The first one is resolving power, which is defined as the minimum $\mathrm{p} /$ difference that can be resolved by IEF, as shown in Equation 2.1 [38].

$$
\Delta \mathrm{p} I_{\min }=3 \sqrt{\frac{\mathrm{D} / \mathrm{E}}{\left(\frac{\mathrm{dpH}}{\mathrm{dx}}\right) /\left(-\frac{\mathrm{d \mu}}{\mathrm{dpH}}\right)}}
$$

Where in Equation 2.1, D is the diffusion coefficient of the anlaytes, $E$ is the applied electric field strength, $\mathrm{dpH} / \mathrm{dx}$ is the $\mathrm{pH}$ gradient, and $\mathrm{d} \mu / \mathrm{dpH}$ is the change of analyte mobility against $\mathrm{pH}$. For certain analyte species, the diffusion coefficient and mobility change are fixed constants; therefore, electric strength and $\mathrm{pH}$ gradient are the main contributors to the resolution of an IEF separation. The higher electric field that is applied, the less steep the $\mathrm{pH}$ gradient will be, and the better resolving power will be obtained. Electric field strength is dependent on the external power voltage and also the separation dimensions; $\mathrm{pH}$ gradient is 
dependent on the carrier ampholyte that is being used. In general, the more amphoteric species with closer $\mathrm{p} /$ values in a carrier ampholyte, the smoother $\mathrm{pH}$ gradient would be formed along the applied electric field.

Another key criterion in IEF is separation efficiency. A traditional terminology to evaluate a separation technique is theoretical plate number, which is defined by the discrete plate numbers required to achieve a designed separation efficiency at the equilibrium stage. This concept was originally used in packed distillation column evaluation before being adopted to chromatography based separations due to having a similar mechanism [104]. The estimation of theoretical plate numbers $(\mathrm{N})$ can be described by Equation 2.2:

$$
\mathrm{N}=16 \frac{\mathrm{T}_{r}^{2}}{\mathrm{w}^{2}}
$$

Where $T_{r}$ is the retention time of the peak and $w$ is the peak width at the base [105].

However in modern IEF, theoretical plate number was replaced by another terminology - peak capacity. It was defined for the first time by Giddings and Dahlgren in 1971, as the maximum resolvable components number [38]. The principle was similar to theoretical plate numbers, but the retention time was replaced by separation length, which is a characteristic factor of IEF device. A theoretical peak capacity $\left(n_{t}\right)$ can be calculated by Equation 2.3, where $F$ is Faraday's constant, $R$ is the gas constant, $T$ is the temperature, and $L$ is the separation length. Similar to the minimum resolved $\mathrm{pl}$, peak capacity is proportional to the square root of electric field strength, separation length, and $\mathrm{pH}$ increment.

$$
n_{t}=\sqrt{\frac{-F E\left[\frac{d q}{d(p H)}\right]\left[\frac{d(p H)}{d x}\right] L^{2}}{16 R T}}
$$

Peak capacity can also be calculated using another method. In some types of IEF, the peak intensity profile is available to obtain, and the peak capacity can be easily estimated by Equation 2.4 [106-108]. This number is based on the experimental 
results; therefore, we use $\mathrm{n}_{\mathrm{e}}$ to distinguish it from theoretical peak capacity in Equation 2.3.

$$
n_{e}=1+\frac{L}{w}
$$

Where $w$ is the peak width taken at $4 \sigma, \sigma$ is the standard deviation of the intensity peak. This $4 \sigma$ is consistent with Equation 2.4, which is expressed as the square root of 16. From both Equation 2.3 and 2.4, separation length is the dominant variable of peak capacity. Generally, longer separation distances among the IEF anode and cathode will offer more space for different types of analytes. However, this length cannot be infinitely increased, instrument capability and cost should always be considered in an IEF design.

Focusing/separation time is another key factor that should be considered in an IEF process. Fundamentally, the entire process is dependent on the transport phenomena of analyte species in the system. The governing equation is shown as Equation 2.5 [88].

$$
\frac{\partial C}{\partial t}=\frac{\partial}{\partial x}\left(D \frac{\partial C}{\partial x}-E \frac{z D F}{R T} C\right)
$$

Where $C$ is analyte concentration, $D$ is the diffusion coefficient of the analyte, $z$ is the net charge of the analyte, $E$ the electric field strength, $F$ is Faraday's constant, $\mathrm{T}$ is temperature, $\mathrm{x}$ is the distance along the $\mathrm{pH}$ gradient, and $\mathrm{t}$ is process time.

For fixed analyte species and temperature, separation time is proportional to distance along the $\mathrm{pH}$ gradient. It is one of the main motivations for IEF device miniaturization, which will be discussed in detail in a later section. Besides, increasing electric field strength can also shorten separation time.

\subsection{Main IEF Categories}

\subsubsection{IEF Prototype}

As mentioned in Chapter 2.2, the earliest IEF prototype was reported by Kolin in Chicago during 1954. The device set-up can be seen in Figure 2.2, a U-shape tube 
with square section was used for separation. In the two end parts connected to the U-shape section, the device was filled by a pH 9.6 buffer; in the U-shape section, the tube was filled with $\mathrm{pH} 2.6$ buffer. Sucrose was used as an accessory chemical to increase the acid buffer density so it could stay at the bottom of the U-shape section. Protein samples were introduced in the intermediate zone ( $\mathrm{M}$ in Figure 2.2). After the electric field was applied, the entire device would serve as both a concentration function in the left half and a dilution function in the right half, depending on the direction of movement of the acid and base buffers. This research introduced the "concentration effect" of amphoteric analytes sandwiched by an acid buffer and a base buffer for the first time. Differing from conventional mobility difference based electrophoretic separation, analytes were sorted by their intrinsic character--isoelectric $\mathrm{pH}$ value, in the concentration half of the device [2]. Later on the same device was used for protein mixtures including hemoglobin, cytochrome $\mathrm{C}$, catalase and collagen, with the use of buffer range from $\mathrm{pH}$ 3.3-7.7 [109]. The establishment of natural $\mathrm{pH}$ gradient was numerically examined by Svensson in the 1960s, which showed the confidence of IEF to be used for amphoteric molecules separation [102]. In later work, Nguyen demonstrated the use of standard amphoteric buffer mixture in $\mathrm{pH}$ gradient formation: an Ampholine mixture, which contains 50-500 amphoteric constituents was oriented by an electric field and then formed a linear $\mathrm{pH}$ gradient. Strong acid and basic solutions ( $\mathrm{KOH}$ and $\mathrm{H}_{2} \mathrm{SO}_{4}$ in cathode and anode, respectively) were applied as electrode solutions to stabilize the $\mathrm{pH}$ gradient during the analytes focusing process [110]. This type of $\mathrm{pH}$ gradient were getting finer after more amphoteric species were included as Ampholine components; therefore, analytes with smaller $\mathrm{p} /$ difference were able to be resolved. Rosa group applied modified narrow range Ampholine ( $\mathrm{pH}$ 6.5-7.5) to hemoglobin variants separation, up to 70 variants were successfully resolved over a $30 \mathrm{~mm}$ separating distribution [111]. Since then, a prototype of amphoteric molecules moving toward their isoelectric point and being concentrated within a $\mathrm{pH}$ gradient and electric field was roughly established as an isoelectric focusing concept. This concept of concentrating molecules was surely 
able to be applied for amphoteric molecules sorting or separating purposes. Millions of works had been conducted based on this concept by then. And the development of IEF started to follow either device modification or $\mathrm{pH}$ gradient modification routes.

\subsubsection{Polyacrylamide Gel IEF}

In IEF prototypes, pH gradient was successfully established and the amphoteric analytes had proven separation and concentration based on their $\mathrm{p} /$ values; however, with the presence of counter IEF phenomena such as diffusion and electrophoretic migration, a method to keep analytes focus longer is required. Among those counter IEF factors, diffusion plays an important role in IEF resolving power and focused band width. From Equation 2, the diffusion coefficient (D) is proportional to the minimum $\mathrm{p} /$ difference $(\Delta \mathrm{p} / \mathrm{min})$; in other words, IEF resolving power can be increased by reducing $D$. With a fixed IEF device, increasing medium viscosity is the only way to lower down $D$ and therefore is the first investigatory priority after the birth of IEF prototypes. Polyacrylamide gel as a universally applied IEF medium was first introduced to IEF by Leaback and Rutter in 1968 [5]. The experimental apparatus used is described in Figure 2.3: unpolymerized acrylamide monomer solution was poured into an $18 \times 8 \times 0.2 \mathrm{~cm}^{3}$ well to form a gel slab after polymerization. Electrode solutions were placed at the two edges of the well and connected with carbon or platinum electrodes. To run the IEF process, polymerized gel was soaked with carrier ampholytes and the sample was loaded into the middle of the gel. A lid was applied on top to prevent evaporations. In this work, the porous gel material structure served as a sieve, to make analytes travel longer distances during separation, and thereby increase the analytes diffusing time. Results demonstrated that protein focused zones had sharp band shapes. After this pioneer work, Righetti et al. published a work using narrow $\mathrm{pH}$ range carrier ampholytes with acrylamide gel for human hemoglobin variants separation [112]. Acrylamide gel was cast into a column, and the post-focused gel could be cut into pieces by different proteins focused locations for post sample analysis. Isoelectric points of focused hemoglobin variants were determined by measuring 
the $\mathrm{pH}$ of gel eluates. Rowley et al. adapted Righetti's method to investigate hemoglobin variants separation in polyacrylamide filled, $10 \times 0.4 \mathrm{~cm}$ I.D. glass tubes. For post-focusing analysis, gel with focused species was scanned by
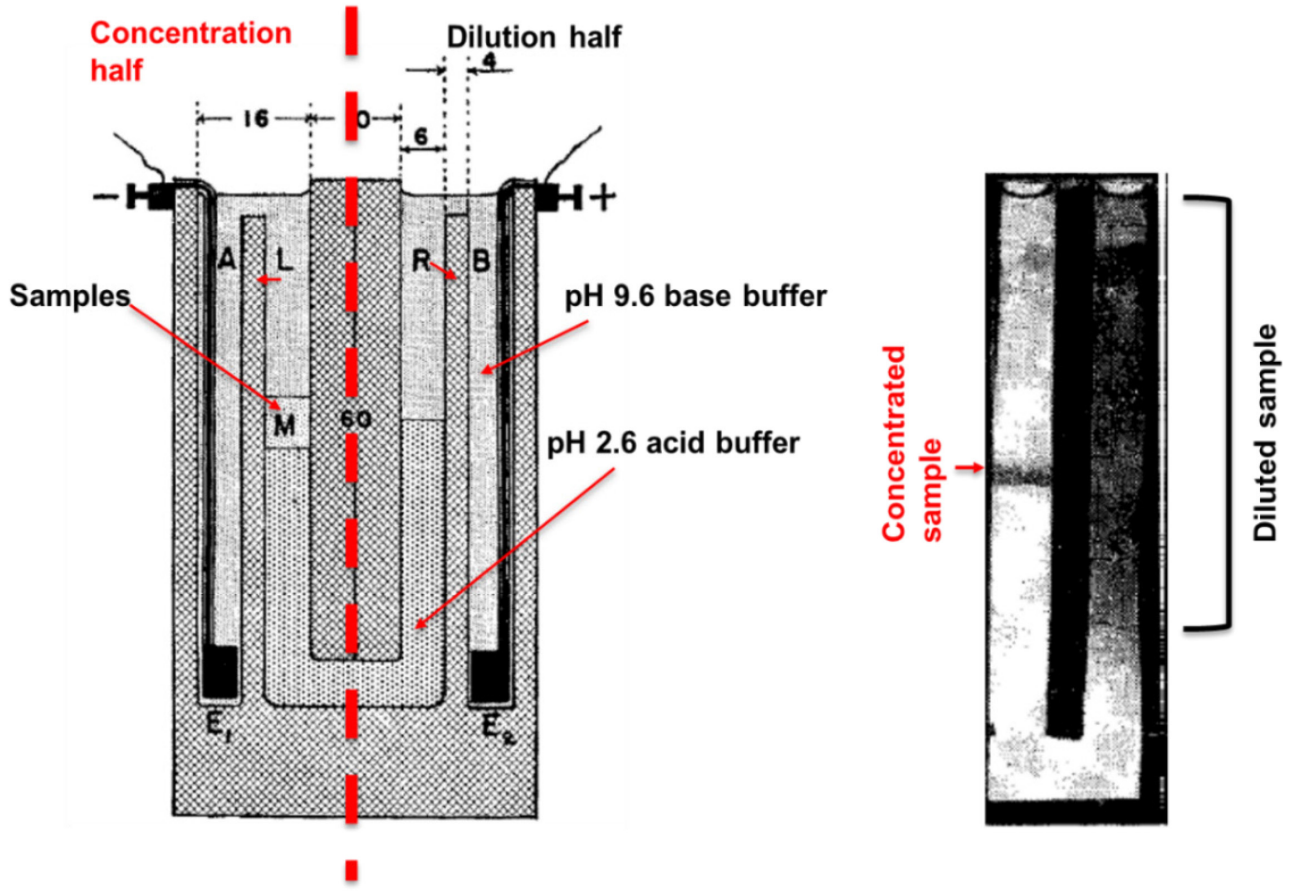

Figure 2.2 Device demonstration of the earliest IEF prototype Reprinted from Kolin, A., Separation and Concentration of Proteins in a pH Field Combined with an Electric Field. The Journal of Chemical Physics, 1954. 22(9): p. 1628-1629. [2], with the permission of AIP Publishing. (See Appendix B)

spectrophotometer and each focused zone was shown as a peak in the scan profiles [113].

The introduction of gel medium greatly improved analytes band shape and brought highly reproducible focusing protocols to IEF. Also in those IEF works, only the gel part was disposable, whereas all the other components of the device were fixed. This feature pushed the slab gel IEF to the commercial level. In general, a gel IEF instrument has three main components including the gel chamber, electrodes, and electrode solution reservoirs that are directly connected to the electrodes. The chamber shapes can be either cylindrical or rectangular, and gel can be either premade or polymerized inside the chamber [114-119]. These days, there is plenty of 
commercial slab gel IEF instruments (such as Bio-Rad Ready Gel ® System and Agilent 2100 Bioanalyzer) and pre-made ready-to-use gel (such as Life-Science Criterion ${ }^{\mathrm{TM}}$ and Ready $\mathrm{Gel}^{\circledR}$, Thermo Fisher Novex) are commercially available, which makes gel-IEF techniques still being used for the same bulk scale commercial protein separation work.

Couples of post-IEF detection techniques can be utilized for polyacrylamide IEF. In the earliest IEF analysis, focused sample zones in gel were cut into pieces and then placed into solvents for sample concentration measurement. To achieve better visualization, the gel could be stained with different types of dyes, including organic dyes, silver stains, negative stains, and fluorescent stains [57]. This staincut-analysis has certain standard operation procedures to follow and thus provides good reproducibility. However, in some procedures (e.g. gel cut, wash, and regeneration) "endless" time to run the sequence is requires. Advanced post-IEF analysis methods including UV-vis detection and fluorescent can make the conventional labor intensive procedures replaced by computer controlled detectors, with the assistants of analysis software, post-IEF work can be more efficient. Overall, the gel stain-cut-analysis method is quite adaptive for some bulk, less time

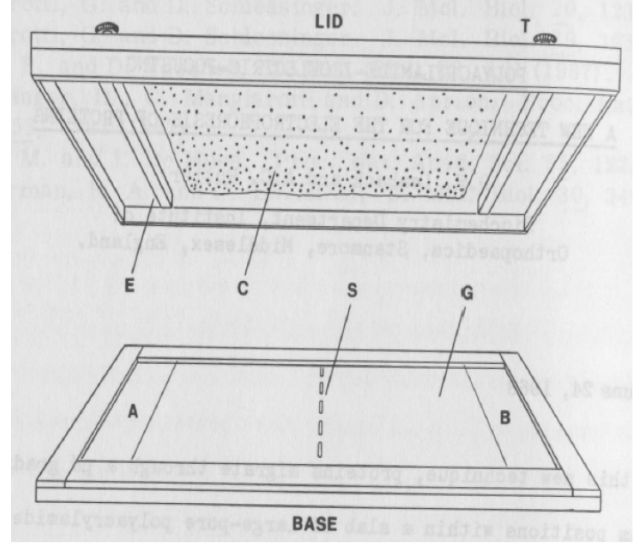

Figure 2.3 Demonstration of slab acrylamide gel IEF. Reprinted from Leaback, D. Polyacrylamide-isoelectric-focusing a new technique for the electrophoresis of proteins. Biochemical and biophysical research communications, 1968. 32(3): p. 447-453 [5]. Copyright (1968), with permission from Elsevier (See Appendix B). 
sensitive proteomic cases. And the automatic detection method shows privilege in clinical and laboratory cases which require more information from samples.

Since the time that polyacrylamide gel IEF was first demonstrated, it has revealed great potential as a reproducible and robust IEF technique. However, polyacrylamide gel IEF requires a long operation time due to the slow migration of analytes inside of gel pores. Also, the gel preparation steps are labor intensive, and the general apparatus scale requires a large amount of analytes to be used in the operation. Therefore, this technique is currently only practical for bulk separation and analysis.

\subsubsection{Capillary IEF}

As another device modification route of IEF prototype, capillary IEF (CIEF) began development in the early 1980 s as the result of pursuing high IEF resolving powers. The first CIEF work was the extension of gel IEF. Baker et al. modified the experimental set-up of horizontal polyacrylamide gel IEF, gel components were filled into a glass capillary instead of rectangular slab gel chamber. About 15 proteinases were detected within a $\mathrm{pH}$ gradient spanning from $\mathrm{pH} 3$ to 10 [120]. A much advanced clEF work was reported by Hjerten in 1985. The highlight of this work was the introduction of a commercial high performance electrophoresis (HPE) instrument to cIEF. Using the same glass capillary, a carrier ampholyte mixture was injected to establish the $\mathrm{pH}$ gradient. After each IEF run, the focused samples were mobilized to a UV-vis HPLC detector to be analyzed. The mobilization operation could either be done chemically, which introduced acid/base species at the anode/cathode end of the capillary to push the sample electrophoretically within the $\mathrm{pH}$ gradient to the detector end; or done physically, which employed conventional HPLC pump to pressurize the focused zone toward the detector. The focusing time could be shortened by replacing the polyacrylamide gel with free

solution inside of the capillary. This would, however, possibly cause the band to be broadened [121]. 
Similar to gel IEF, capillary IEF also has electrodes and electrolyte reservoir components; however, capillary is the main IEF element in cIEF. Due to small capillary volumes (down to micrometer I.D.), both samples and solutions required to fill the separation space can be considerably reduced; therefore, CIEF is more economically efficient. Other than that, the maximum resolvable species, in terms of peak capacity, can be significantly improved by magnitude due to the raised separation length. Typically, a cIEF instrument can have a peak capacity of up to $10^{3}$, which makes cIEF a good candidate for complex protein mixtures analysis [64, 122].

Regarding post-IEF detection methods for CIEF, UV-vis and fluorescent are the mainstream techniques being employed. In the early stages of cIEF, either UV-vis or fluorescent detector was operated without visualization. Generation of $\mathrm{pH}$ gradient and concentration of analytes happened in the closed capillary column, and post-focused analytes were pushed through the detector with original incapillary distributions kept. Detectors output analytical results as electrograms while transferring absorption responses of analyte species, concentrations or other optical properties into intensities. In this case of scenario, UV-vis and fluorescent are working on very similar bias with the only difference being the optical absorption mechanism. However, in newly developed cIEF techniques, whole column imaging detection (WCID) was introduced to help simplify the IEF process [123-125]. This technique employed a short, optical transparent column instead of using conventional capillary coils. A charge coupled device (CCD) camera was mounted under capillary to replace conventional detectors which mounted at the end of column. In this case, fluorescent detection will offer better quality results with enhanced contrast and signal-to-contrast ratio, due to the intrinsic optical properties of fluorescein.

With present-day highly integrated commercialization and automation level, the CIEF technique is being widely used as an IEF separation benchmark for complex and standardized sample separations in clinical and laboratory areas. Technically, CIEF is able to offer the best resolving power among all of the IEF techniques. The 
shortcomings of cIEF including high cost, and highly skilled labor requirements in operation and data analysis, prevent cIEF from becoming a universal IEF tool. As mentioned previously, the main adaptive areas for cIEF are fine clinical analysis and laboratory, which are not economy and time sensitive

\subsubsection{Miniaturized IEF}

Even though gel IEF and CIEF are well established techniques with robust SOP and sufficient separation resolving power, the unsolved issues including long focusing time, large sample/chemical cost, and highly skilled labor requirements, make these two types of IEF somewhat cumbersome in certain applications. In the meantime of making gel IEF and CIEF more optimized, research focuses started to move toward the development of new IEF techniques. Miniaturized IEF ( $\mu \mathrm{IEF})$ was born in this new IEF era, and aimed to aid issues relating to the instrument size. The first miniaturized IEF prototype was based on a modification of a conventional clEF work in 1999 [3]. To reduce the IEF scale, the original capillary was replaced by a $40 \mathrm{~mm}$ long, $100 \mu \mathrm{m}$ wide and $10 \mu \mathrm{m}$ height quartz chip. The detailed structure and dimension of the chip can be seen in Fig 2.4. The microchannel on the chip was created by photolithography and chemical etch. The chip was mounted under a linear CCD array and the UV light transmission through the channel was collected. Separation was tested by low molecular mass $\mathrm{p} /$ markers and myoglobin as model samples. Results reflected a resolving power of $0.03 \mathrm{pH}$ unit. The main contribution of this work was the demonstration of miniaturization possibilities in IEF. With adequate resolving power, IEF separation length could be reduced to $4 \sim 5 \mathrm{~cm}$ from the conventional 12 60 cm capillary. Besides, microfabrication technique such as photolithography were introduced to ensure the accuracy of the IEF fine feature design. After this work, $\mu \mathrm{IEF}$ experienced rapid developments due to the fast detection and low sample requirements scenario. 
A
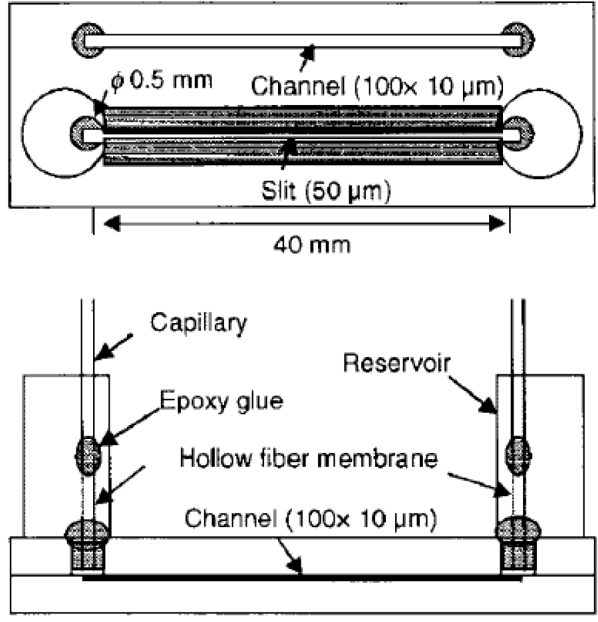

B

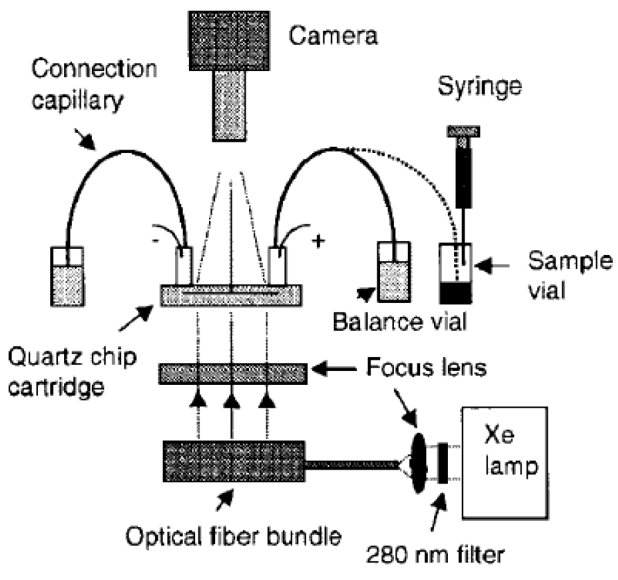

Figure 2.4 First $\mu$ IEF chip structure and the experiment sequence set-up. Reproduced from Mao, Q. and J. Pawliszyn, Demonstration of isoelectric focusing on an etched quartz chip with UV absorption imaging detection. Analyst, 1999. 124(5): p. 637-641[3]. http://dx.doi.org/10.1039/A809756I. With permission of The Royal Society of Chemistry (See Appendix B).

The main change of $\mu$ IEF from conventional gel IEF and clEF is the entire device size. Due to the development of microfabrication techniques, small features such as patterned electrodes and deliberate channels can be fabricated down to the nanometer level, which facilitates the miniaturization of IEF devices. Mostly, the major component of $\mu \mathrm{IEF}$, separation spaces, are created following the photolithography masking-mold feature developing-replica molding route. To build up the separation channel, Poly (dimethylsiloxane) (PDMS) is one of the most popular materials due to its inexpensive cost, optical transparency, and ready-togo casting recipe. A typical microfabrication-PDMS based $\mu \mathrm{IEF}$ device can be obtained by the following: 1) a channel pattern is created via soft lithography onto a glass slide/silica wafer (photolithography masking), 2) after developing the photoresist layer, a positive channel feature can be created onto the slide/wafer (mold feature developing), 3) To form the PDMS layer with replica channel feature, polymerized liquid PDMS elastomer and curing agent mixture are poured onto the 
featured slide/wafer. After the liquid fully polymerizes, the PDMS layer can be peeled off with the replica channel feature (replica molding). The obtained PDMS layer with features can be fully sealed later onto a glass/silica surface via plasma adhesion. In Cui et al.'s work, this described route was typically demonstrated. The obtained PDMS layer can be seen in Fig. 2.5. Channel structure was in dimensions of $2 \mathrm{~cm}$-long, $300 \mu \mathrm{m}$-wide and $5 \mu \mathrm{m}$-deep. The inlet and outlet of the channel were created using a bio-punch, and the $\mathrm{pH}$ gradient was generated using Pharmalyte $^{\mathrm{TM}}$ with up to $200 \mathrm{~V}$ DC applied. IEF performance was tested using green fluorescent protein (GFP) and r-phycoerythrin (PE). Due to the optic transparency of PDMS, the protein focusing behaviors could be observed directly under a fluorescent microscope, which made a huge improvement in terms of

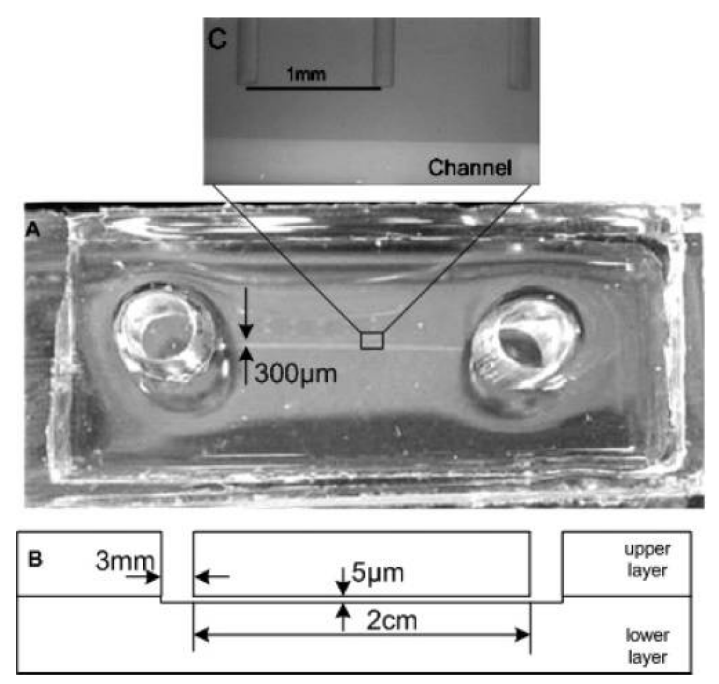

Figure 2.5 Device photo and geography dimension of PDMS channel $\mu \mathrm{IEF}$ device. Reprinted with permission from Cui, H., et al., Isoelectric Focusing in a Poly(dimethylsiloxane) Microfluidic Chip. Analytical Chemistry, 2005. 77(5): p. 1303-1309.[4] Copyright (2005) American Chemical Society (See Appendix B).

visualization.

With the assistance of microfabrication techniques, $\mu$ IEF channel structure and material, device design, and scale can easily be manipulated by adjusting the fabrication parameters during manufacture, which give $\mu \mathrm{EF}$ much better diversity 
than conventional gel IEF and cIEF. A short summary is shown in Table 2 regarding the channel structure, material, and $\mathrm{pH}$ gradient type. In fact, each of those properties can be combined freely for assigned applications making this customization capability the unbeatable advantage of $\mu \mathrm{IEF}$. 
Table $2 \mu$ IEF channel structure, material and $\mathrm{pH}$ gradient type

\begin{tabular}{|l|l|}
\hline Channel structure & Straight [3, 4, 91, 126-129]; \\
& Triangular [130] \\
& House shoe [131] \\
& T-shape [132, 133] \\
& Parallel [134, 135]; \\
& Droplet [136] \\
& Multidimensional [67, 69, 127, 131, \\
& 134, 137, 138] \\
\hline Channel material & Quartz [3, 91] \\
& Glass [67, 69, 92, 137, 139, 140] \\
& PDMS [4, 130, 133] \\
& PMMA [127, 134, 138] \\
& SU-8 photoresist [93] \\
& Polyester (Mylar) [126] \\
& Nonwoven fabric [129] \\
\hline pH gradient type & CA type [4, 91, 131, 133, 137] \\
& Immobilized pH [130, 134, 140] \\
& Natural pH without CA [126] \\
\hline
\end{tabular}

\subsection{IEF Materials}

\subsubsection{Materials for pH Gradient Generation}

Establishment of the $\mathrm{pH}$ gradient is the key process for the entire IEF work. Only with the $\mathrm{pH}$ gradient along the separation space are amphoteric analytes possible to be focused spatially. In the very first IEF prototype and its early follow-up work, $\mathrm{pH}$ was artificially manipulated by diffusion of non-amphoteric buffer with different $\mathrm{pH}$ value $[2,109,141]$. This deliberately formed $\mathrm{pH}$ gradient was confined by slow diffusion speed under the electric field and buffer solution type to secure $\mathrm{pH}$ stability; therefore, it could only be applied to simple amphoteric analytes with known $\mathrm{p} /$ values. To achieve better separation resolving power and use IEF for 
unknown samples, a finer and smoother $\mathrm{pH}$ gradient is desperately in need. Svensson first introduced a "natural pH gradient" concept in 1961[102]. In this work, a principle of building up a favorable $\mathrm{pH}$ gradient system was defined as "pairs of buffers containing the same ion species". To approach this principle, a pH gradient was attempting to generate by adding weak acid and base ampholytes to the separation instead of a strong acid and base buffer. In the anode and cathode ends, proton and hydroxide ions were consistently generated by electrolysis, which maintained the lowest and highest $\mathrm{pH}$ at $\mathrm{pH}$ gradient ends. Between electrodes, weak acid/base ampholytes were positively or negatively charged by protons or hydroxides from the electrode and therefore moved toward the position where the ion concentration could reach to equilibrium state again. Once the movement of ampholytes stopped, the final $\mathrm{pH}$ gradient would be dictated by ampholytes' $\mathrm{p} /$ values. The significant contribution of this work is the demonstration of the direction for $\mathrm{pH}$ gradient stabilizing. In general, the more acidic and basic the components presented into the system are, the more stabilized the $\mathrm{pH}$ gradient will be that is formed. The natural $\mathrm{pH}$ gradient work enlightened a route of pursuing finer and smoother $\mathrm{pH}$ gradient for maximizing IEF resolving power. Later, many weak acid/base amphoteric species pairs were found and explored for $\mathrm{pH}$ gradient establishment. Eventually in 1964, a mixture of amphoteric species was commercialized as carrier ampholytes (CAs) solutions in accordance with a Sweden patent, and was granted the US Patent no. 3485736 in 1969. With highly standardized commercial control, CAs could easily be used to generate designated $\mathrm{pH}$ gradient with decent stability. In modern IEF, commercial CAs are still the most reliable solution for linear, smooth $\mathrm{pH}$ gradient usage.

The most common 4 types of commercial CAs are Pharmalyte and Ampholine (product of LKB-produkter AB, purchased by Pharmacia in 1986), Bio-Lyte (product of BioRad), and Servalyt (product of Serva). All of these CAs are composed of amphoteric molecues and their corresponding isoforms (e.g. oligoamino, oligo-sulfonic acid (for low $\mathrm{pH}$ ), and succinylmethyl group (for high $\mathrm{pH})$ ). As mentioned previously, the more amphoteric species in CAs, the smoother 
the $\mathrm{pH}$ gradient that can be generated. Normally, commercial CAs contain hundreds of chemical entities and more than one thousand isoforms. Righetti reviewed all four types of CAs with different $\mathrm{pH}$ range in the market, and the information data is summarized in Table 3 [21]. From the compounds and isoform numbers, Servalyt CAs have the most varieties; therefore, they are supposed to be the CAs with the best performance. Servalyt also offers an extremely basic $\mathrm{pH}$ range from 9-11, which is not covered by other CAs. However, Servalyt CAs are not the most commonly used in IEF work. The reason for this could be an economic issue. Comparing with Servalyt, Pharmalyte has comparable compounds and isoforms numbers, and the cost is considerable lower. These benefits make Pharmalyte the leading CAs in IEF applications. It should be pointed out that compounds and isoforms numbers in different $\mathrm{CAs}$ bands vary in different $\mathrm{pH}$ ranges, for example, Pharmalyte contains more compounds but less isoforms than Servalyt in $\mathrm{pH}$ range of 4-6. Researchers should select CAs in order to achieve the best CAs performance for the specific IEF applications. 
Table 3 Compounds information of commercial CAs

\begin{tabular}{|c|c|c|c|}
\hline Brand & $\mathrm{pH}$ range & $\begin{array}{c}\text { Compounds } \\
\text { numbers }\end{array}$ & Isoforms numbers \\
\hline \multirow{3}{*}{ Ampholine } & $2-4$ & 105 & 446 \\
\cline { 2 - 4 } & $4-6$ & 80 & 325 \\
\cline { 2 - 4 } & $6-8$ & 80 & 326 \\
\cline { 2 - 4 } Bio-Lyte & $8-10$ & 29 & 85 \\
\cline { 2 - 4 } & $2-4$ & 84 & 383 \\
\cline { 2 - 4 } & $4-6$ & 66 & 436 \\
\cline { 2 - 4 } & $6-8$ & 32 & 237 \\
\hline \multirow{3}{*}{ Pharmalyte } & $8-10$ & 43 & 136 \\
\cline { 2 - 4 } & $2-4$ & 245 & 821 \\
\cline { 2 - 4 } & $4-6$ & 217 & 812 \\
\cline { 2 - 4 } Servalyt & $6-8$ & 123 & 476 \\
\cline { 2 - 4 } & $8-10$ & 58 & 102 \\
\cline { 2 - 4 } & $2-4$ & 227 & 1201 \\
\cline { 2 - 4 } & $4-6$ & 199 & 703 \\
\hline \multirow{3}{*}{} & $6-8$ & 126 & 306 \\
\hline
\end{tabular}

${ }^{*}$ data referred from [21]

In addition to CAs, which are prepared for linear type $\mathrm{pH}$ gradients, there is another way to establish $\mathrm{pH}$ gradient. This method is based on amphoteric molecules covalently linked to an anti-convection medium to maintain the local $\mathrm{pH}$ and then cast medium with different $\mathrm{pH}$ into a gradient. Due to the unchanged local $\mathrm{pH}$ in each medium section, the casted $\mathrm{pH}$ gradient was named "immobilized $\mathrm{pH}$ gradient". The first immobilized pH gradient for IEF application was reported by Bjellqvist et al. in 1982, a set of acryloyl monomers called Immobiline were introduced in that research [142]. Each species of monomer contains only two weak acid/base to generate a $\mathrm{pH}$ gradient in a very narrow range (less than 0.2 ) within an electric field. After co-polymerization with acrylamide/bis monomer, the narrow pH gradient would be immobilized. By linearly casting different Immobiline species, any $\mathrm{pH}$ gradient between $\mathrm{pH}$ 3-10 could be built up. Compared with $\mathrm{pH}$ gradient generated from CAs, immobilized $\mathrm{pH}$ gradient was staircase type. However, by casting many stair-steps $\mathrm{pH}$ segments together, the final gradient could be pseudo liner. The most remarkable advantage of immobilized $\mathrm{pH}$ gradient 
was the stability, and since free-flow solution was kept away from the IEF system, the established $\mathrm{pH}$ gradient would not decay by any transport driven force; therefore, it could last longer time than the CAs type gradient. Like the development of CAs, immobilized $\mathrm{pH}$ monomer was commercialized soon after. To make the product more user-friendly, the latest immobilized $\mathrm{pH}$ monomers were made into polyacrylamide gel strips, which can be cut into any size and then be casted orderly to obtain a designated $\mathrm{pH}$ gradient.

It should be noted that even though immobilized pH gradient gel (IPG) is highly commercialized, and fairly easy to use with great stability, the size limit is still an issue hindering immobilized $\mathrm{pH}$ gradient from IEF miniaturization. In today's world IPG is staying active only in bulk IEF separation applications.

\subsubsection{IEF Media}

In earlier IEF work, steps of $\mathrm{pH}$ gradient establishment, sample loading, and IEF separation were processed simultaneously in the separation space of the IEF device/instrument. This type of IEF operation was defined as "free-flow" IEF because all of the reagents including sample could move freely in the IEF separation space with an applied electric field. The mix-everything-together method did shorten the IEF prepared steps and made the operation less complicated, however, transport driven forces such as convention and elecroosmosis would noticeably disturb the IEF once it reached to the equilibrium stage. Accordingly, issues including $\mathrm{pH}$ gradient drift and focused band broadening could happen. To solve this intrinsic problem of free-flow systems, efforts such as increasing solution viscosity, and adding a coating layer onto the surface of the IEF path had been investigated $[4,67,128,143,144]$. These detailed solutions will be explained in the next chapter.

On the other hand, gel IEF as an alternative IEF technique, uses gel as an IEF media. The monolith gel with millions of opening pore structures could allow big analyte molecules (e.g. protein molecules) to pass through. In the meantime, due to the twists and turns in gel pores, the travel time of analytes or other molecules 
take longer than with the free-flow system. This character would secure focused sample from severe diffusion and therefore offered enough time for the postanalysis of the focused samples. As mentioned in Chapter 2.3.2, polyacrylamide is the mostly used gel material in IEF and the physical characteristics have been thoroughly explored. In a polyacrylamide system, two key variables, T and C, are widely adopted to determine the pore structure of polymerized gel. T, which is defined as the total percentage concentration of monomer in the gel, can be estimated using Equation 2.6:

$$
\mathrm{T}=\frac{100 \text { (Total acrylamide and bis) }}{\text { Total gel volume }} \%
$$

And $\mathrm{C}$, which is defined as the crosslinker (bis) percentage of both acrylamide and the crosslinker, can be described in Equation 2.7:

$$
\mathrm{C}=\frac{100(\text { bis })}{\text { Total acrylamide+bis volume }} \%
$$

In a commercial acrylamide/bis monomer regent, common $\mathrm{T}$ values vary from $2 \%$ - 40\%, and $C$ values are usually in 2.5\% (acrylamide/bis $=37.5: 1$ ), $3.3 \%$ (acrylamide/bis=29:1), and 5\% (acrylamide/bis=19:1). By changing $T$ and $C$ numbers, the pore size of a polyacrylamide gel can be manipulated. $T$ is inversely proportional to the pore size, the higher percentage gels (higher $\mathrm{T}$ ), the smaller pores. Equation 2.8 can be used to estimate gel pore radius $\left(r_{p}\right)$ with known $T$ value. The relationship of $C$ to pore size is complex. Generally, the minimum pore size occurs when $C$ is about $5 \%$ (a 19:1 gel). Decreasing $C$ results in a more open pore structure because there are fewer crosslinker molecules. The $T$ and $C$ numbers should be chosen per the analytes size. Small molecules such as DNA can use high $T$ and $C$ values, while big molecules such as proteins require small $T$ and $C$ to be used in gel.

$$
r_{p}=231 \times(100 T)^{-0.51}
$$




\subsubsection{Accessary Materials}

Except $\mathrm{pH}$ gradient generating agents and medium, there are a couple of accessary materials for an IEF system. These materials include electrode solutions, additive chemicals, and mobilizer (only for cIEF). Electrode solutions are employed at the anode/cathode end of the IEF separation space to fix the lowest/highest $\mathrm{pH}$ value along the established $\mathrm{pH}$ gradient. Usually strong acid/base solutions (e.g. phosphoric acid and sodium hydroxide) are selected at two ends of the $\mathrm{pH}$ gradient. The role of different electrode solutions were investigated by Naydenov in 1992 [145]. In his research, ten and six electrodes solutions were used in anode and cathode, respectively. The anode electrode solutions included strong acids $(\mathrm{HCl}$, $\mathrm{H}_{3} \mathrm{PO}_{4}, \mathrm{HClO}_{4}$, and $\mathrm{H}_{3} \mathrm{PO}_{4}$ in ethylene-glycole), weak acids (glycine, triethylenetetramine-ampholytes, En-ampholyte with $\mathrm{pH} 6.30$ and $\mathrm{CH}_{3} \mathrm{COOH}$ ) and a neutral $\mathrm{H}_{2} \mathrm{O}$ solution; the electrode solution at the cathode side included a strong base $\left(\mathrm{NaOH}, \mathrm{NaOH}\right.$ in ethylene-glycole), a weak base $\left(\mathrm{NH}_{3}\right.$, triethylenetetramineampholytes with $\mathrm{pH} 8.8$ and $\mathrm{PEHA}$ ) and a neutral $\mathrm{H}_{2} \mathrm{O}$ solution. Results demonstrated that using a strong acid/base for the electrode solutions could maximally maintain the developed $\mathrm{pH}$ gradient, while other types of electrode solutions made $\mathrm{pH}$ gradient narrower than it was supposed to be. The reason used to explain this was the proton/cation concentration did not change severely compared with bulk strong acid/base volumes at the two $\mathrm{pH}$ gradient ends. It should be mentioned that this is an expectation of electrode solutions in modern IEF. When working with miniaturized gel, electrode solutions and electrode strips may not be required due to thickness of the gel layer [146].

Additive chemicals are the regents which can facilitate IEF separation, maintain $\mathrm{pH}$ gradient, and improve separation efficiency. To facilitate IEF separation, a widely applied chemical type used is surfactant. The introduction of surfactant into IEF helps to release protein-protein or protein/surface interactions, and thereby allowing the protein precipitate phenomenon happening during the focusing process to possibly be alleviated. In the case of gel IEF, surfactant can also facilitate proteins bursting out from gel pores [147]. To maintain the $\mathrm{pH}$ gradient, 
surface coating is a working method. A chemical coating onto the IEF separation space surface can manipulate surface properties such as zeta potential, hydrophilicity, and surface resist, to thereby alleviate external counter-IEF forces generated onto the IEF medium-surface boundary [148-152]. In the case of CIEF, chemical mobilizer can help the focused zone to move electrophoretically toward detector direction, and make the analytes better analyzed by detector $[63,121$, $125,143,144]$.

There are also a few other accessary chemicals being used in IEF work. For example, in bulk gel IEF, it would be beneficial to add a mesh sealing to the separation space, to prevent gel dehydration [5]. In free flow IEF, Nafion ${ }^{\circledR}$ is applied in the two electrode sides to separate direct contact between electrode and electrode solutions [153]. Due to the selectively ion primitive feature of Nafion $\circledast$, the hydrogen ion could be transported through the film while electron conduction was prevented. This modification lessened the ion strength by 40 fold during IEF $\mathrm{pH}$ gradient establishing process and therefore introduced less disruption. Thus, a more stable, longer-lasting $\mathrm{pH}$ gradient could be built up. In summary, applying accessory materials is optional and method dependent to an IEF work. Introducing accessory materials may grow the IEF device complexity in the manufacturing aspect, but if applied properly, accessory materials can sufficiently increase separation efficiency.

\subsection{Difficulties and Issues in IEF}

\subsubsection{Focusing Time and Applied Voltage}

Equation 5 in Chapter 2.2 demonstrated the relationship between IEF separation time and separation space along a pH gradient. Conventional IEF usually has separation space at the scale of $10 \mathrm{~cm}$ or higher; therefore, a typical separation time is in the hour timescale $[112,113,120,150,154,155]$. To shorten focusing time, reducing IEF separation space is one of the effective ways. That is also one of the motivations of IEF device miniaturization and has been proven by many previous $\mu \mathrm{IEF}$ researchers. With a typical separation space in $1 \mathrm{~cm}$ or less size, 
focusing time was promptly reduced down to 20 min or less [4, 91, 93, 132]. It will be valuable to further investigate the lower limit of IEF devices in the separation space dimension aspect; however, the exploration of the IEF scale limit has reached a bottleneck due to fabrication techniques. The conventional optical lithography molding technique has a feature resolution at the $\mu \mathrm{m}$ scale [156-158], considering the other components such as sample inlet/outlet, electrode solution reservoirs and sampler, the final separation space will be much larger than the microfabrication limits, and this issue makes the currently smallest $\mu \mathrm{EFF}$ in $1 \mathrm{~mm}$ size [159]. To further investigate the IEF size limit, newer techniques having better resolution than optical lithography arenecessary, and a manipulating machine with finer operation capability is required.

Another concern in conventional IEF is the applied voltage. The electric field is the main external driving force for IEF to achieve a full sample separation. In IEF operation, DC is directly applied along the separation space through the anode and cathode. To complete a separation and make the sample species focused into sharp bands, at least $10^{2} \mathrm{~cm} / \mathrm{V}$ electric field strength is required [68, 87, 160]. The electric field strength is proportional to the space dimension that is along the field. Depending on the separation space size, DC power can to reach up to $10^{3} \mathrm{~V}$ for a typical CIEF, and even for $\mu$ IEF $10^{2} \mathrm{~V}$ will be necessary. The high DC power will not only make high energy consumption, but will also generate heat to the separation space, and lead to unexpected phenomena such as band distortion and broadening [115, 161-164]. Besides, extremely high DC power applied in an IEF operation is always a safety concern. To lower down the external DC, IEF miniaturization is again essential. The current $\mu \mathrm{IEF}$ are operated at the $\mathrm{DC}$ range of 50 500 V [88], comparatively safer than ClEF, but the further decrease of DC voltage to a safe level (e.g battery driven IEF) will always be a motivation for miniaturized IEF work.

\subsubsection{Unstable $\mathrm{pH}$ Gradient}

Since the establishment of IEF in the 1950s, the capability of IEF in real applications have been widely investigated and sample separation trials on 
different proteins and peptides have been done. Along with the promising properties of IEF, issues regarding the $\mathrm{pH}$ gradient were being exposure. The CA type $\mathrm{pH}$ gradient was noticed to be unstable as a function of time and voltage due to the immigration of free ions, and as the result, focused amphoteric sample zones could decay or become dislocated towards the electrode [38, 165]. Nguyen et al. artificially made instable $\mathrm{pH}$ gradient scenarios and summarized the possible reasons [155]. In their work, all the observations including $\mathrm{pH}$ gradient drift, CA, and focused zones drift were following the direction toward the cathode side. These artificial phenomenon occurred in the case of 1) CA replaced by buffer, and 2) electrode solutions replaced by buffer. During their experiment, other drift phenomenon were also observed when the electric field was just applied and the temperature changed. By summarizing the experimental results, they concluded that $\mathrm{pH}$ gradient would be stabilized by reversing the mass transports in IEF system, including suppressing EOF, selecting proper electrode solutions, and shortening the diffusion time. In Mosher's work, a series of simulations were performed regarding the effect of electrode solution concentrations to the $\mathrm{pH}$ gradient. Results verified that the progressive loss of $\mathrm{pH}$ gradient end components was the reason for gradient drift, and by changing the electrode solution concentrations, drift phenomenon could be manipulated. As possible solutions, the electrode solution reservoirs should be much larger than separation space, and the ratio of electrode solutions concentration and current density should be maximized. Also, a physical barrier (ion exchange membrane) would help to cease the ampholyte migration.

In general, unstable $\mathrm{pH}$ gradient is still a main concern for the development of IEF techniques. In any IEF design, a balance of spacing, the $\mathrm{pH}$ gradient chosen, and unwanted mass transport prevention should be seriously considered. 


\subsection{Current Trends and Future Directions of IEF Technology}

\subsubsection{Current Trends and Future Directions}

The IEF development timeline has been fully illustrated in the previous chapter. In short, the entire IEF history has been through a large chamber, long tube, slab gel, capillary tube, and microchannel stage. This shows the general trend of IEF development: from large and complicated instruments to small and easy-to-use devices; from long time and labor intensity operations to programmed and automatic runs; from huge sample requirements to small sample loadings; from single detection to orthodox analysis/multiple functions integration. The most important attributes are lower detection limits and better resolutions.

The work from Zubarev's group can be used as a good example for future generation IEF. A MJ-cIEF (multi-junction capillary isoelectric focusing) technique featured a multi-junction sampler and an immobilized staircase $\mathrm{pH}$ gradient [58, $66,166]$. The device setting is based on capillary IEF, for sampling, a 6-way valve is employed to decrease sample requirement (typical $2 \mu \mathrm{L}$ needed), also different samples can be processed with multiple injections. For $\mathrm{pH}$ gradient set-up, vials with fixed $\mathrm{pH}$ are put along the capillary flow direction. Nafion film is used for materials exchange between vials and capillary. This technique can reduce sample requirements as well as analysis time (since no time is necessary for $\mathrm{pH}$ gradient establishment). For applications, both proteins and peptides are proven to be preseparated. With an integrated desalting cartridge in their most advanced version, even plasma can be directly analyzed as sample for proteins prefractionation.

\subsubsection{Surface IEF and Its Application for Protein Separation}

As a chemical analysis method, isoelectric focusing (IEF) based electrophoresis and related blotting techniques are a mainstream protein analysis technique. In a certain $\mathrm{pH}$ gradient within an externally applied electric field, positive or negative charged amphoteric protein species will migrate toward different $\mathrm{pH}$ values, and then cease moving at the place where $\mathrm{pH}$ is equal to their isoelectric points. Since proteins have unique isoelectric points, protein mixtures can be separated into 
focused bands. To establish a $\mathrm{pH}$ gradient within an externally applied electric field, carrier ampholytes (concept envisioned by Kolin [109]) and immobilized pH gels (IPG) (concept introduced by Reghetti [142]) are the two main routes. The final option is via natural $\mathrm{pH}$ gradients generated through water electrolysis at the anode and cathode [102]. Conventional IEF is conducted in bulk commercial instruments or in customized bulk gel slabs, and is not qualified to rapid analyses with small sample volumes. LOC development offered an opportunity to solve the issue. For instance, capillary isoelectric focusing (CIEF) is a combination of conventional IEF and capillary electrophoresis instrumentation [160]. Compared to large-scale IEF set-ups, clEF can be conducted in centimeter scale chips and is considered portable. cIEF also has good resolution, comparable to conventional bulk IEF, and provides narrow focusing zones. Fused silica capillaries or channels are utilized with cIEF to contain the carrier ampholytes and allow for free solution IEF. During focusing, the capillaries do limit axial diffusion of proteins. In a typical clEF operation, proteins can either be focused during transportation towards the detection point, or be focused and then moved past the detection point along with the focused zones. However, these capillaries prevent the easy recovery of IEF focused bands; the focused bands must be mobilized via electrophoresis or pressure, and the bands broaden with dispersion prior to recovery. CIEF is considered as an effective, high-resolution method to separate closely related proteins with minor structural differences, such as Hemoglobin variants [167], transferrin, and peptides [160]. However, the stability and recoverability of proteins during CIEF indicates space for improvement in low concentration, sensitive protein analysis applications requiring subsequent MS-MS, TOF, or similar analysis.

Channel IEF is a closely related technique, which has further reduced sample volumes and labor. Channel IEF utilizes microchannels from either hard etching or reverse mask replicating; the channels are filled with IEF gel media or carrier ampholyte solution, and IEF can be achieved upon applying an external electric field. Hofmann first minimized clEF onto a small glass slide in 1999 [168] by utilizing wet etching on a glass chip to create capillary tube-like channels $7 \mathrm{~cm}$ in 
length. Pharmalye ${ }^{\circledR}$ (GE brand of carrier ampholyte) with $1 \mathrm{kV} / \mathrm{cm}$ electric field focused Cy-5 peptides, which were detected with laser-induced fluorescence (LIF). The final separation was a combination of chemical (IEF) and hydrodynamic electroosmotic flow (EOF) mobilization, which later proved to have a negative resolving factor for IEF [169]. Yager et al. reported an on-chip "channel" IEF application for protein separation in 2001 [126, 170]. In their experiment, a 40-mmlong $\times 1.27$-mm-wide $\times 0.354-\mathrm{mm}$-deep channel was fabricated by laser ablation micromachining of poly (Mylar). Gold/Palladium foil was electrodeposited on channel ends and functioned as electrodes for IEF. A natural $\mathrm{pH}$ gradient was generated by water electrolysis. This simple operated design was susceptible to the natural $\mathrm{pH}$ gradient being disturbed by perturbations to the applied voltage (high applied voltages cause electrolysis bubble generation). Cui et al. investigated carrier ampholyte (broad range 3-10 Pharmalyte ${ }^{\circledR}$ ) based IEF in PDMS channels for fluorescent green fluorescent protein (GFP, p/ 6.0) and phycoerythrin (PE, p/ 4.4). Methylcellulose (MC) was added to the solution to suppress EOF. GFP and PE were fully separated in the $2 \mathrm{~mm}$ channel and focused bands were held stationary in up to $50 \mathrm{~V} / \mathrm{cm}$ applied electric fields [4]. In the last few years, channel IEF chips have been optimized to be highly-integrated and tailored for certain separation purposes - continuous separation [67, 93, 137], and batch sample sorting $[69,138,171]$, etc.

However, all existing IEF devices require either large volumes of carrier ampholytes and electrode buffering solutions or require complicated device microfabrication. In my $\mathrm{PhD}$ research, a novel surface enabled isoelectric focusing (SIEF) concept will be introduced and interrogated. A microscale surface IEF chip was developed using 1 step photolithographic fabrication technologies combined with a surface-patterning instrument. Preliminary data illustrates the viability of sIEF process in a 2-D gel line 300 microns in length. Commercial Pharmalyte ${ }^{T M}$ carrier ampholyte solution was used for $\mathrm{pH}$ gradient generation, and the generated $\mathrm{pH}$ gradient was characterized by $\mathrm{pH}$ sensitive fluorescent dye FITC Isomer I, $\mathrm{pH}$ insensitive TRITC (reference), and fluorescent $p /$ markers with different $p /$ values. 
SIEF is attractive because it requires nanoliter reagent volumes and relies upon a highly reproducible, easy, and quick fabrication process. This unique and creative micro-scale surface approach has the potential to enable more efficient specialty protein separations from ultra-small sample volumes. The platform is reusable and simple to operate, and potentially able to replace current commercial and classic instrumentation.

\subsubsection{Quick Outlook of Future IEF}

In the future, bulk IEF and micro IEF will still co-exist due to different application requirements. For bulk IEF, the improvement of commercial instrumentation should be the main focus. Good existing examples are Bio-Rad Rotofor ${ }^{\circledR}$ and Mini Rotofor Cells, which have dimensions of $16.5 \times 45.7 \times 22.8 \mathrm{~cm}$ and are capable of discerning 20 species with up to $35 \mathrm{~mL}$ sample loading. Compared with conventional gel IEF instrument, Rotofor ${ }^{\circledR}$ and Mini Rotofor have equivalent throughput with much reduced sized and simplified operation. For micro IEF, the miniaturization will stay a mainstream trend, and it will be significant to further explore the miniaturizing limit for IEF. In the meantime, micro IEF will play as one of the dimension in a type of integrated mutil-dimensional separation chip. Also, new fabrication techniques should be involved in micro IEF design, to decrease the cost and manufacture complexity. 


\section{Surface Printing Technique for LOC-IEF Device Fabrication ${ }^{1}$}

\subsection{Introduction}

In the past two decades, materials were able to be exploited and manipulated under micro or sub-micro scale due to the rapid development of nanotechnologies, research areas such as medical, biomaterial and bioanalysis which requires miniaturized patterning and tests were very much benefited. Atomic force microcsopy (AFM) as one of commonly used imaging technology, was found to be able to carry and pattern micro scale organic materials via its micro cantilever probe in the late $20^{\text {th }}$ century [49], this discovery opened a window of customized surface patterning: various of materials including organic chemicals and metals were investigated to be patterned on arbitrary surfaces such as metal, quartz, glass and silicon [47, 51, 52]. Nevertheless, as a tool explicitly designed for imaging process, AFM does not have designed features that facilitate micropatterning. Patterning shape, size and working duration were restricted by AFM cantilever. To make the surface patterning more operable and controllable, a technique called fluidics enhanced molecular transfer operations (FEMTO) was developed in the early $21^{\text {st }}$ century. This type of instruments usually composed of liquid reservoirs to ensure working duration and fluidic channels to deliver sample to the surface. Plus, the instrument is easily to be customarily designed. At the beginning stage, FEMTO instruments were designed for liquid sample dots/dot matrix patterning. Later on the utilization of this technology was extended to variety of materials: from organic sample proteins $[77,79,81]$, DNA $[75,76]$, polystyrene [61], quantum dots (QD) [62] to inorganic sample silver nanoparticle [73], carbon nanotubes [74].

\footnotetext{
${ }^{1}$ The material contained in this chapter has been accepted for publication in Electrophoresis.

DOI:10.1002/elps.201600565
} 
As illustrated in Chapter 1, new tools and techniques can offer plenty of opportunities for new platform designs of IEF devices. FEMTO tool has already been proved to feasibly print liquid materials into more complicated shapes than simple dots/dot matrix under sub-micro level. In this chapter, we are continuing to investigate the capability of line-shape patterning using FEMTO tool, which will further enable the possibility of fabricating an IEF gel in micro scale.

\subsection{Materials and Methods}

\subsubsection{Materials and Instrument}

A commercial FEMTO tool Nano eNabler ${ }^{T M}$ (Bio science) is employed to investigate surface pattern. This tool enables precise pattern-printing of diverse fluids on arbitrary surfaces [24-30]. It includes a controllable sample stage (XY travel: $50 \mathrm{~mm}$; $X Y$ resolution: $20 \mathrm{~nm}$; $Z$ travel: $50 \mathrm{~mm}$; $Z$ Resolution: 100nm), surface patterning tool (SPT) holder, microscope with CCD camera and chamber with humidity control. The overlook of instrument can be seen as Fig 1.2 in Chapter 2 , and the structure of SPT, as well as microscope images can be seen in Fig 3.1

A $40 \% \mathrm{w} / \mathrm{v}$ stock solution for acrylamide monomer was made from 29:1 ratio acrylamide and bis-acrylamide powder (Sigma-Aldrich, St. Louis, MO, USA), tetramethylethylenediamine (TEMED) and glycerin were obtained from PlusOne (New York, NY, USA). E-pure water with $18.2 \Omega \cdot \mathrm{cm}$ resistivity was purified with an EMD Millipore Simplicity Ultrapure 185 water system (Billerica, MA, USA). Ammonium persulfate, $\left(\mathrm{NH}_{4}\right)_{2} \mathrm{~S}_{2} \mathrm{O}_{8}$, (APS, PlusOne, New York, NY, USA) at varies of concentrations water solution $(10 \%, 15 \%$ and $20 \% \mathrm{w} / \mathrm{v})$ was used as a polymerization catalyst for acrylamide solution. This mixture is referred to as the acrylamide/bis-acrylamide/APS solution below.

For surface pre-cleaning a UV-ozone generator (BioForce UV/OZONE PROCLEANER ${ }^{\mathrm{TM}}$ system) was applied. This instrument removes from the SPT and device surface any organic molecules contamination such as sample residues, grease and finger prints. It secondarily influences the surface hydrophobicity. To quantify this impact on hydrophobicity, contact angle tests of acrylamide/bis- 
acrylamide/APS solutions on the IEF device substrate were performance via Kruss G-10 (Hamburg, Germany) drop shape analyzer with five repeats under three different UVO treatment times of the device substrate. The data was processed via Drop Shape Analysis 1.0 software.

E-pure water was loaded as sample for printing trial run, in order to inspect the drawing capability. After the water trial a printing was practiced with the delivery of acrylamide/bis-acrylamide/APS mixture solution.

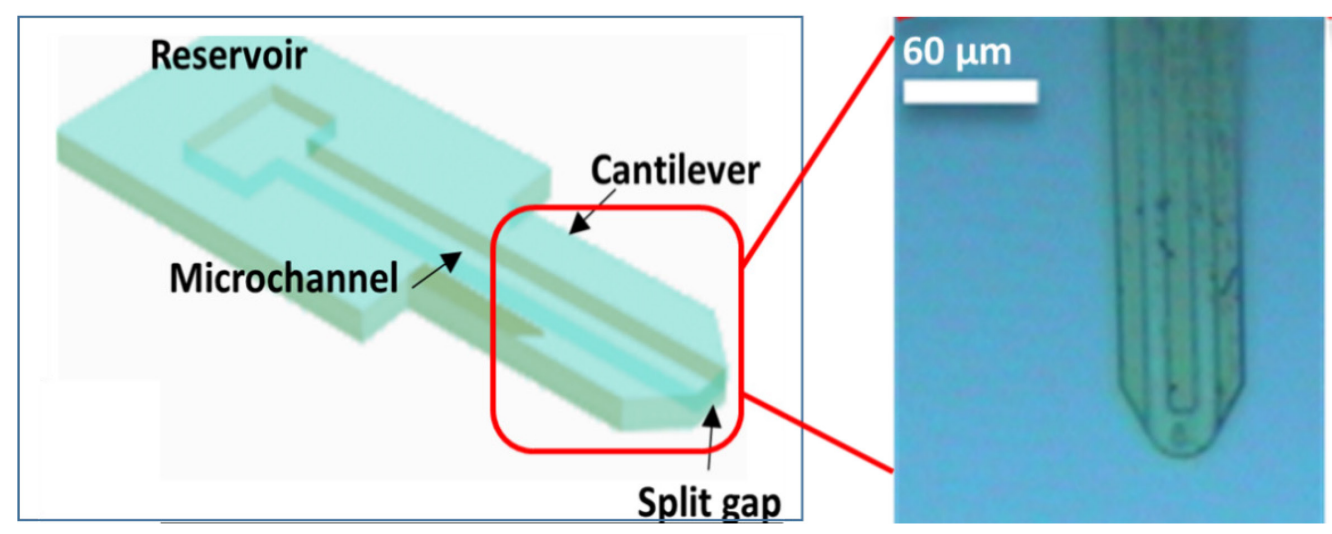

Figure 3.1 The SPT structure (left) and microscope image of SPT tip (right) illustrating the microchannel for fluids to flow from the reservoir to the printing tip.

\subsection{2 sIEF Electrodes Microfabrication}

Electrodes are in direct contact with the IEF gels as the conduit for the electric field application. In sIEF, planar electrodes were designed onto a $3 \times 7 \mathrm{~cm}^{2}$ glass chip. The mask design includes a pair of L-shaped electrodes with $300 \mu \mathrm{m}$ gap between the parallel electrode region, and a pair of circle contact pads with $5 \mathrm{~mm}$ diameter. The contact pads were used to connect to wire leads from the power supply. These micro-patterned gold electrodes (100 $\mu \mathrm{m}$-wide spaced, $300 \mu \mathrm{m}$ apart) were prepared using soft photolithography followed by electro-vapor deposition (Ebeam). The photolithography process includes substrate cleaning, preparation, photoresist application, exposure and developing steps. A $3 \times 7 \mathrm{~cm}^{2}$ microscope glass slide was pre-cleaned three times in acetone, isopropanol, and distillated water, before spin-coating with PR-1000A photoresist at 1500 rpm for 40s. After 
spin coating, the glass substrate was prebaked at $120{ }^{\circ} \mathrm{C}$ for 90 seconds. An electrode pattern was transferred from a computer designed, hollow caved mask onto the photoresist layer via UV exposure (see Figure 3.2). The glass substrate was directly covered by the mask followed by UV exposure at doses of $210 \mathrm{~J} / \mathrm{cm}^{2}$. After exposure, the substrate was post baked again at $120^{\circ} \mathrm{C}$ for 3 minutes. Finally the patterned photoresist was developed using RD-6 developer and the exposed photoresist was washed away. To obtain the patterned metal electrodes, E-beam was used to deposit metal on the exposed glass surface. Titanium was used as adhesion layer and gold was deposited on the top of Ti layer, with $0.500 \mathrm{kA}^{\circ}$ (500 $\mathrm{nm}$ ) deposition rate. The obtained metal layers contain $5 \mathrm{~nm}$ Ti followed by 150 $\mathrm{nm}$ Au (SOP can be found in Appendix A). Photoresist and excess metal were removed via a lift-off process by acetone sonication at medium power for 10 minutes.

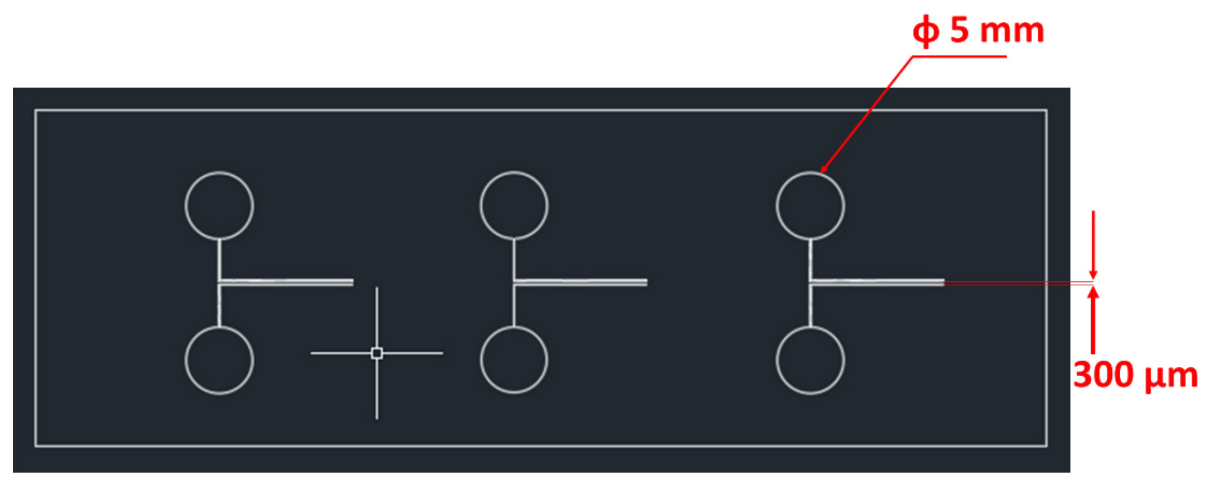

Figure 3.2 AutoCAD design of sIEF drive electrode pattern

\subsubsection{Surface Printing}

From previously reported research, Nano eNabler ${ }^{\mathrm{TM}}$ was mostly used to generate dots or dot matrix. In our research we want to explore the limitation of the instrument to generate lines. Before printing, liquid sample was loaded via two different ways: front loading and back loading based on the loading position of surface pattering SPT. The operation of front loading is to dip the SPT tip into a sample droplet and let sample attached on the tip by capillary force; the other operation, back loading, is to fill the reservoir in the back of SPT with liquid sample, 
so it can be delivered to drawing surface though microchannel in SPT. The drawing was also tested via two different ways: one the method is to make a dot column with dense dots arrangement, and then let the dots merged by surface tension, the second method is similar like brush printing or ink-pen writing directly on the surface with sample as paint or ink. The Z-direction control allows SPT tip to reach to the patterning surface, and Y-direction vertical movement of sample stage enables the line formation.

\subsubsection{Optimizing Printing Conditions}

Series of optimization factors were conducted including 1) surface hydrophobicity, 2) humidity of printing environment, 3) sample loading way in SPT and 4) polymerization time. For surface hydrophobicity modification, UV-ozone cleaner was applied as mentioned previously, degree of surface modification was investigated by varying treatment time. Printing humidity was controlled by humidifier that directly connected to the chamber in Nano eNabler ${ }^{\mathrm{TM}}$, chamber was closed during printing process. Both front loading and back loading were examined

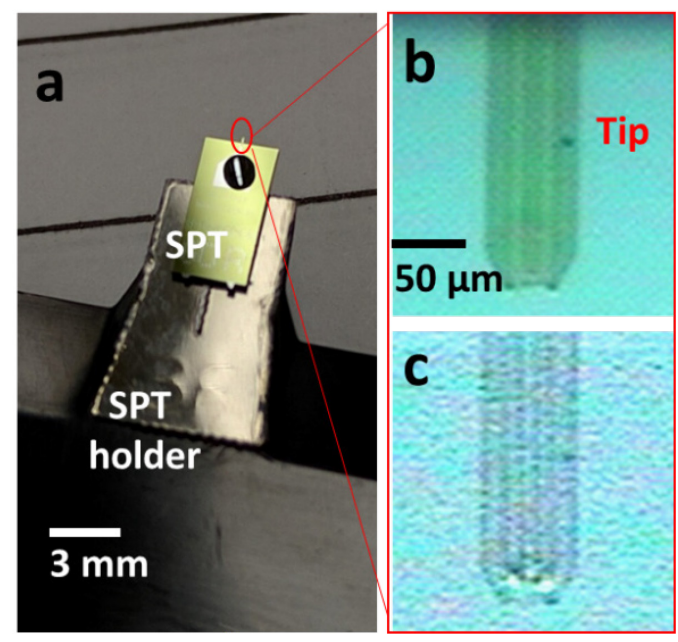

Figure 3.3 Sample back loading of the SPT. a) Magnified image of loaded SPT with the sample appearing as a dark droplet in the reservoir near the tip. b) Microscope images of an unloaded SPT tip demonstrating the channel that connect the reservoir to the tip and c) Microscope image of a loaded tip with fluid within the channel ready to be printed. 
for line printing as sample loading investigation. The gelation degree of printed line was investigated by applying different polymerization time.

\subsection{Results and Discussion}

\subsubsection{Sample Loading}

The Nano eNabler ${ }^{\mathrm{TM}}$ instrument achieves surface printing by utilizing attributes of the surface patterning tool (SPT), which is comprised of a larger flat square of silicon substrate with a reservoir etched onto the back and a $150 \mu \mathrm{m}$-long cantilever that extends below the surface on the front side. The cantilever has with a tapered end; the reservoir and the cantilever are connected with a channel. During instrument operation, there are two techniques for sample loading for printing/patterning: front and back loading. Back loading sample printing is more controllable because the printing/patterning solutions are stored in the back reservoir of the surface patterning tool (SPT) then flow down the channel to SPT tip for printing onto the surface. Back printing works similar to a modern ink pen. Preliminary experiments showed this loading method worked well with low to mid viscosity liquid samples such as water (Figure 3.2). However, when printing high viscosity materials, such as acrylamide, the in-situ gelation properties prevented flow from the reservoir along the SPT channel to the tip. Thus, front sample loading was utilized similar to a dip quill pen. A small acrylamide droplet $(0.1 \mu \mathrm{L})$ was pipetted on the glass slide adjacent to the experiment working area. The SPT was maneuvered to the acrylamide droplet, the tip immersed, then lifted and positioned for subsequent printing.

Precision and reproducibility of sample printing was ensured via NanoWare ${ }^{\mathrm{TM}}$ software that automated control of the cantilever tip movements. The software allowed total range of motion of $50 \mathrm{~mm}$ of $X Y$ (with controllable 2 200 $\mu \mathrm{m} / \mathrm{s}$ speed) travel and $45 \mathrm{~mm}$ of total $Z$ travel. Step resolution in $X Y$ and $Z$ directions is $20 \mathrm{~nm}$. An optical zoom camera allowed real-time monitoring of the tip and surface; the field of view can be magnified between $1.72 \mathrm{~mm} \times 1.72 \mathrm{~mm}$ and $0.26 \mathrm{~mm} \times 0.26$ $\mathrm{mm}$. To aid with reproducibility, relative $X Y Z$ stage position can be saved and reloaded as needed. 
To protect the fragile SPT cantilever tip as it approached the glass surface, the contact pressure was monitored via NanoWare ${ }^{\mathrm{TM}}$ and adjusted as necessary to optimize tip-surface position. Figure 3.3 shows the different pressure levels applied to the SPT tip, which can be visualized by the extent of tip bending. Without
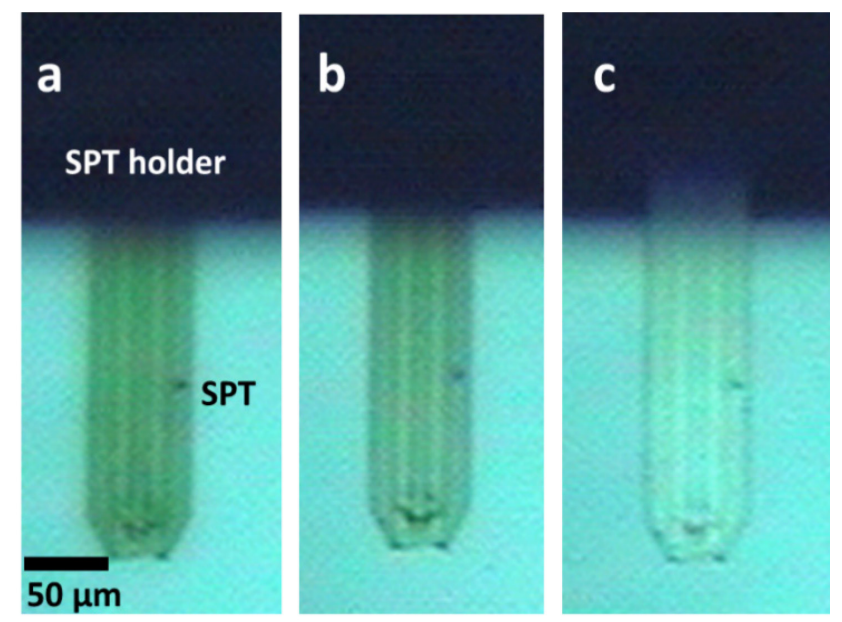

Figure 3.4 SPT tip with varying degrees of contact with the surface. a) No contact between the tip and surface. b) Tip gently resting on the surface and c) Excess pressure on the tip such that it experienced bending.

tip/surface contact, printing is not successful. However, if the tip is bent, the tip tends to break during vector movements. Figure 3.3a shows no tip/surface contact, 3.3b shows optimal tip/surface contact, and 3.3c illustrates tip bending. These visual cues were used to assess optimal tip contact to ensure smooth, reproducible printing as well as extend the SPT lifetime.

Acrylamide gel printing was achieved via front loading of the tip and strategic vector motions to produce printed lines across the parallel microfabricated electrodes on the glass chip. A $0.2 \mu \mathrm{L}$ volume drop of acrylamide was manually pipetted onto the glass slide away from the micropatterned electrode. The SPT cantilever tip was positioned in $\mathrm{XYZ}$, then dipped into the acrylamide drop. The liquid-loaded SPT was then repositioned in XYZ at one electrode edge then was moved linearly (by programming in a start/end position and rate in the software) to deposit a line spanning across the parallel electrodes, as shown in Figure 3.3. 


\subsubsection{Hydrophilicity/Hydrophobicity of the Surface}

Surface hydrophobicity was found to be critical to reproducibly print samples. The extent of surface hydrophobicity can be tailored via UV-ozone (UVO) treatments. The microscope glass slide is primarily comprised of $\mathrm{SiO}_{2}$, making the surface slightly hydrophilic due to the weak bond between $\mathrm{O}$ atoms of $\mathrm{SiO}_{2}$ and the $\mathrm{H}$ atoms of $\mathrm{H}_{2} \mathrm{O}$. However, the adsorption of hydrophobic organic molecules from the environment onto the glass surface can slowly increases glass hydrophobicity over time [53]. UVO treatments are a convenient method to remove organics and thus to recover surface hydrophilicity. In a typical UVO cleaner, there are two wavelengths of UV light generated; $184.9 \mathrm{~nm}$ can be used for ozone generation,

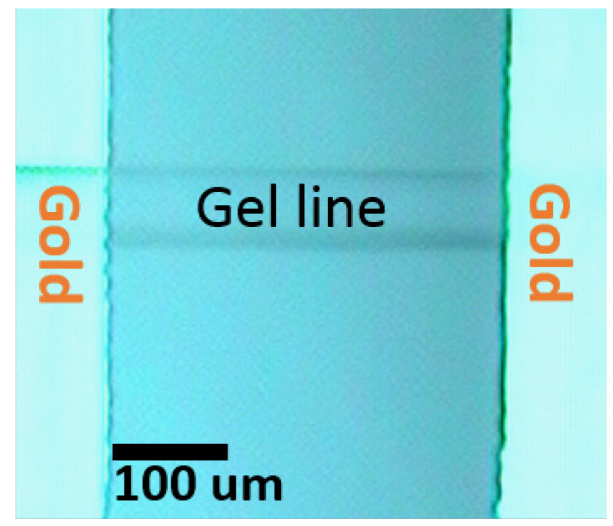

Figure 3.5 Demonstration of acrylamide gel line drew via front loading of SPT and automated linear vector movement of the SPT.

and $253.7 \mathrm{~nm}$ can be used for ozone destruction. As the ozone generationdestruction intermediate product, atomic oxygen, serves as a strong oxidant to react with UV excited organic contaminants on the surfaces to converts them into volatile molecules [55]. This UVO cleaning process enables electrode micropatterned glass-slides to be reused $\sim 50$ times for sIEF tests before failure. In our printing optimization, UVO treatment time dependency was investigated to compare the hydrophobicity/hydrophilicity effects on acrylamide line printing quality. UVO exposure times of $5 \mathrm{~min}, 10 \mathrm{~min}$, and $20 \mathrm{~min}$ were examined and the printed gel line shape was observed under an optical microscope. With 5 min UVO exposure, the glass slide remained hydrophobic such that the liquid-glass surface 
tension broke the printed line into several fragments and the line integrity was broken. As treatment time increased to $10 \mathrm{~min}$, line integrities remained good with uniform width. This result illustrates sample/glass interfacial tension decreased with decreasing surface hydrophobicity such that the printed line shape was not distorted by surface tension. This smooth printed line shape was desirable for subsequent line printing. An extended 20 min treatment time further increased surface hydrophilicity, which resulted a low contact angle phenomenon whereby an irregular, flat printed line was observed. Microscope images of the printed line with $5 \mathrm{~min}, 10 \mathrm{~min}$ and $15 \mathrm{~min}$ UVO treatment can be seen in Figure 3.5. By comparing line quality, surface UVO cleaning times of 10 minutes yielded the most uniform and consistent acrylamide gel line printing.

To verify these microscope observation based UVO treatment time dependency, the contact angles between acrylamide solution and glass surface under different UVO treatment time were tested. Figure 3.5 showed the UVO time-contact angle dependency: as surface treating time increased, the contact angle became smaller, which implicated more hydrophilic glass surface. This result is consistent with the microscope observation. In the meantime, as UVO time increased, the standard deviations of each repeat sets under different test conditions became smaller. This variation of standard deviations are due to the variation of surface purity of glass. As commercial product, the untreated glass slide has plenty of organic impurities such as grease randomly attached on the surface. The short time UVO treatment cannot thoroughly remove those impurities, therefore the contact angle test repeats that performed in different location varies a lot. With longer time UVO cleaning, more impurities on glass surface were removed and therefore the test results on different locations were more consistent with each other. For the reference of experiments in further chapters, another surface, dielectric hafnium oxide $\left(\mathrm{HfO}_{2}\right)$, was also been tested through the same conditions. The surface contact angle results are shown in Figure 3.5. Again, the UVO time-contact angle dependency was similar to the result of glass, longer surface cleaning can maximally regenerate the hydrophilicity of $\mathrm{HfO}_{2}$. However, the standard deviations 
on $\mathrm{HfO}_{2}$ showed better consistency compared with glass due to the much lower contaminations on the cleanroom produced $\mathrm{HfO}_{2}$ layer. It needs to be mentioned that the overall contact angle of $\mathrm{HfO}_{2}$ is little higher than glass, which make lower quality yield of surface printing on $\mathrm{HfO}_{2}$.

\subsubsection{Relative Humidity in the Printing Environment}

Unlike surface property effects, humidity did not substantially influent surface printing quality indicators like width and integrity. However, longer-term stabilization of the 3D gel shape was observed to correlate to printing chamber humidity. Ideal gel line characteristics for sIEF should include 1) straight line with smooth uniform width and 2) shape retention for $\sim 3$ hours to allow time for gelation and subsequent sIEF run. The Nano eNabler ${ }^{\mathrm{TM}}$ instrument can manipulate and monitor relative humidity $(\mathrm{RH})$ in the printing chamber throughout the printing process. To determine optimized humidity conditions, $\mathrm{RH}$ between ambient and

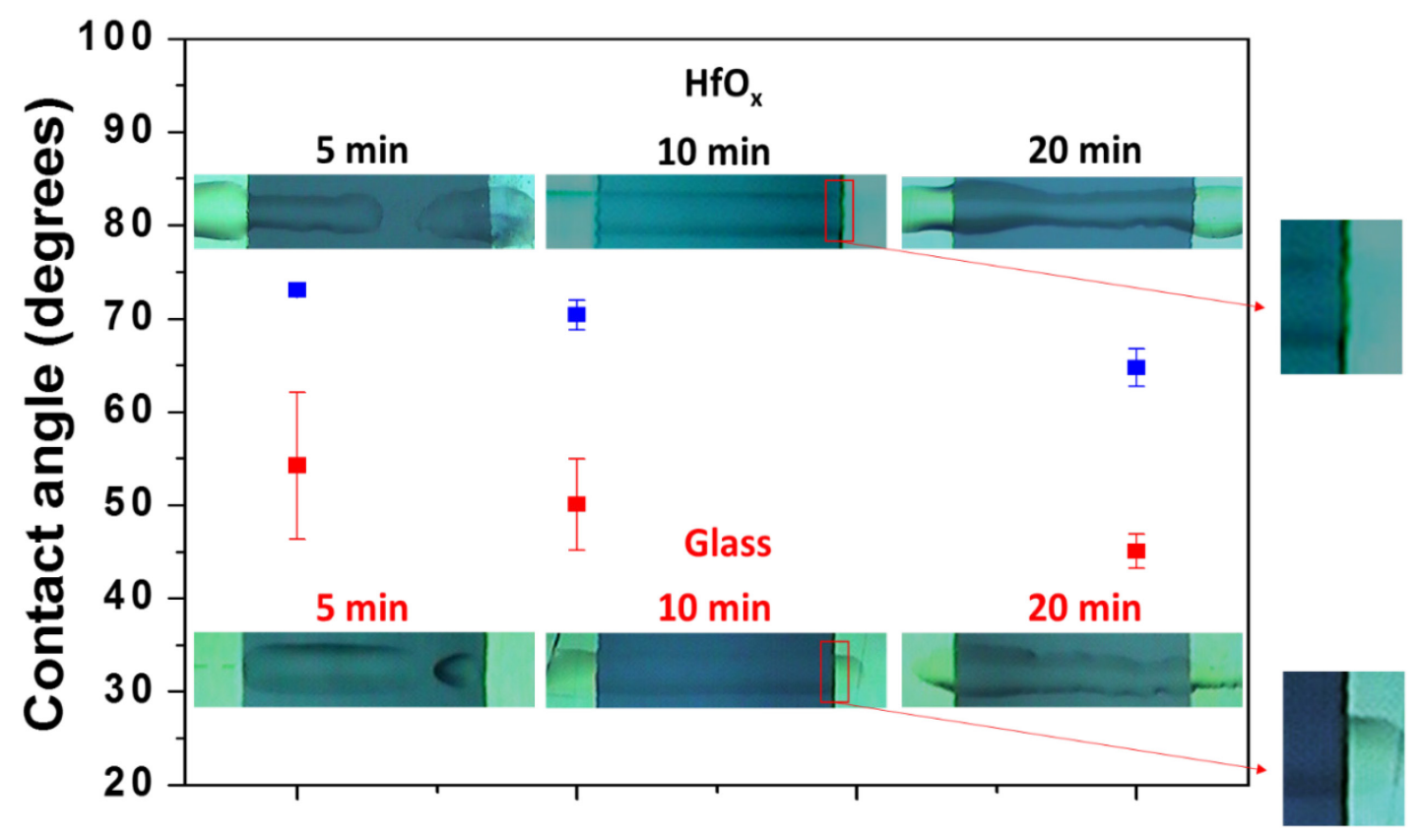

Figure 3.6 Microscope images of surface printed line and surface contact angle data based on both glass and $\mathrm{HfO}_{2}$ surface. Both of the printing and contact angle measurement were based on UVO surface treatment time of $5 \mathrm{~min}, 10 \mathrm{~min}$ and $15 \mathrm{~min}$. For both surface, optimized UVO treatment time are all in $10 \mathrm{~min}$, which is able to maintain the line shape without over-wetting or discontinued. 
$50 \%$ were explored. The $40 \% \mathrm{w} / \mathrm{v}$ acrylamide/bis $(29: 1 \mathrm{v} / \mathrm{v})$ solution was fixed in all the experiments. Figure 3.6 compares gel status in ambient (open chamber enclosure), $15 \%, 30 \%$, and $50 \% \mathrm{RH}$ settings on the module, which were maintained both during printing and for 3 hours after printing. All printings experiments were completed on the same day to ensure consistent room humidity. In the open chamber, ambient conditions control at around $10 \% \mathrm{RH}$, printing was successfully performed, but the gel line experiences rapid evaporation (due to the high air circulation caused by chamber opening) achieving a dried out state in less than 30 minutes. These conditions would not allow sufficient time for acrylamide gelation. Thus, we increased the chamber humidity controller to $15 \% \mathrm{RH}$ with successful printing. Further, the gel line shape remained optimal for the 4 hours observed. Printing remained successful with $30 \% \mathrm{RH}$ setting, however, the gel line began swelling after 1 hour to compromise final gel line quality. To fully explore humidity saturation, a gel line was printed at an extremely high humidity setting of $50 \% \mathrm{RH}$. Condensation was observed such that the whole glass chip flooded within an hour. Thus, $15 \% \mathrm{RH}$ control was utilized for all sIEF line printings. 


\subsubsection{Catalyst Concentration and Related Acrylamide Gelation Time}

Active printing requires the sample to be aqueous, while sIEF run requires a gel state. We engineered around these constraints by mixing the monomeric acrylamide solution with the gelation catalyst (APS, Ammonium polysulfide) just prior to SPT tip loading and printing, then allowed ample time for complete gelation. Previous IEF research utilizes $20 \% \mathrm{w} / \mathrm{v}$ APS aqueous solution added to 29:1 acrylamide:bis-acrylamide solution to achieve gelation ( $0.4 \% \mathrm{w} / \mathrm{v}$ in entire gel) in about half an hour time [172]. Adaption of these same conditions achieved full gelation in less than a minute, leaving insufficient time to print the solution.

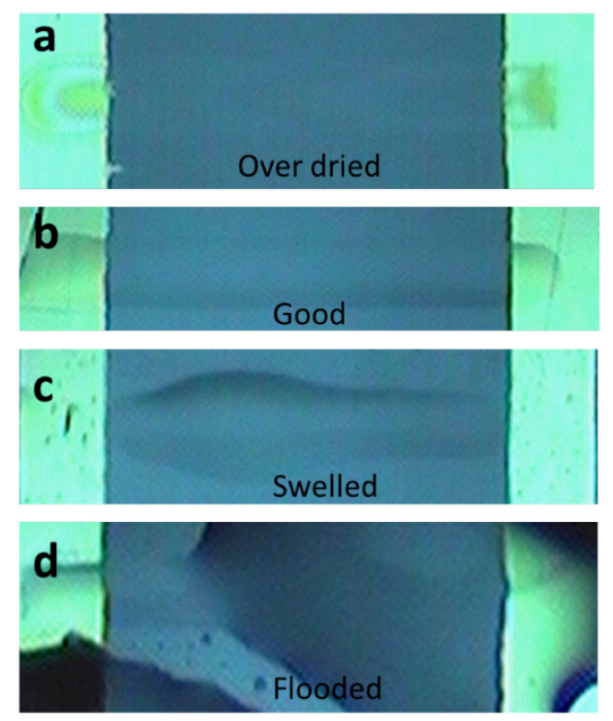

Figure 3.7 Humidity control during printing process. Different chamber humidity were applied include a) No humidity control, gel line get dried out. b) $15 \%$ humidity, line stayed well. c) $30 \%$ humidity, line became swelling with environment humidity increased. And d) $50 \%$ humidity, flood phenomenon observed in glass chip.

To prolong gelation times, we performed line printing with APS concentrations with $10 \% \mathrm{w} / \mathrm{v}$ and $15 \% \mathrm{w} / \mathrm{v}$ APS (0.2 - $0.3 \% \mathrm{w} / \mathrm{v}$ in entire gel). Gel polymerization results are shown in Figure 3.7. With $10 \%$ w/v APS, even though the printing was good, the acrylamide solution did not polymerize within 4 hours. Using an APS concentration of $15 \% \mathrm{w} / \mathrm{v}$, gelation was observed within $2.5 \sim 3$ hours. This enabled ample time to print multiple gel lines. And as mentioned previously, APS 
concentrations at $20 \% \mathrm{w} / \mathrm{v}$ polymerized rapidly and prevented printing. Thus, $15 \%$ $\mathrm{w} / \mathrm{v}$ became the standard solution conditions for all subsequent sIEF experiments.

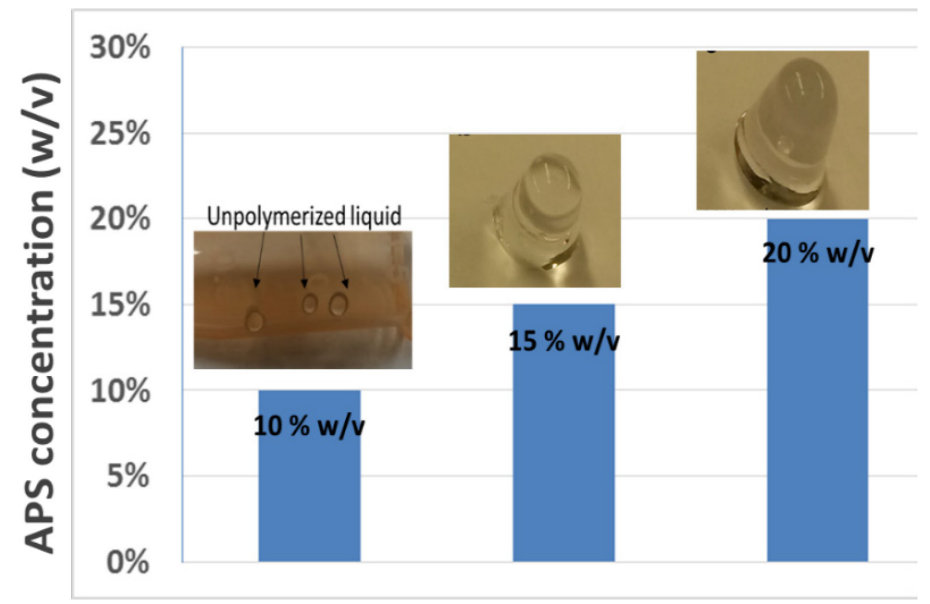

Figure 3.8 Acrylamide gelation photos with different APS concentration. a) $10 \% \mathrm{w} / \mathrm{v}$, b) $15 \% \mathrm{w} / \mathrm{v}$ and c) $20 \% \mathrm{w} / \mathrm{v}$. With $10 \% \mathrm{w} / \mathrm{v}$ APS acrylamide solution is not able to polymerize. Both 15 and $20 \% \mathrm{w} / \mathrm{v}$ will allow acrylamide turn into gel, but only $15 \% \mathrm{w} / \mathrm{v}$ will offer enough polymerization time to finish line printing.

\subsection{Conclusions}

The fabrication of SIEF device, including electrodes fabrication and separating gel printing, was completed by photo lithography, E-beam deposition and nanoprinting techniques. For electrode fabrication, a uniform $\mathrm{Au}-\mathrm{Ti}$ layer with controllable deposition rate was achieved by E-beam depositor. For nano-printing operations, Nano eNabler ${ }^{T M}$ instrument was first time adapted for IEF purposes. Printing conditions of sample loading methods, surface hydrophobic/hydrophilic property, printing humidity and acrylamide gelation were investigated to reinforce gel line shape with good reproducibility. The final recipe was determined to be SPT front loading, 10-minute UV-ozone recovery of surface hydrophilicity, 10\% 20\% relative printing humidity, and the use of $15 \%$ w/v APS with 3 hours of polymerization. As the result, a sIEF device contains two parallel drive electrodes and a straight acrylamide/bis gel line contacted by electrodes was prepared for later sIEF operation. 


\section{Isoelectric Focusing (sIEF) with Carrier Ampholyte pH Gradient $^{2}$}

\subsection{Abstract}

Isoelectric focusing (IEF) is a powerful tool for amphoteric species separations because of high sensitivity, bio-compatibility, and reduced complexity compared to other separation techniques. IEF miniaturization is attractive because it enables rapid analysis, easier adaptation to point of care applications, and smaller sample demands. However, existing small-scale IEF tools have not yet been able to analyze single protein spots from array libraries, which are ubiquitous in many pharmaceutical discovery and screening protocols. Thus, we introduce an in situ, novel, miniaturization approach we have termed surface isoelectric focusing (sIEF). Low volume printed sIEF gels can be run at length scales of $\sim 300 \mu \mathrm{m}$, utilize $\sim 0.9$ $\mathrm{ng}$ of protein with voltages below $10 \mathrm{~V}$. Further, the sIEF device platorm is so simple that it can be integrated with protein library arrays to reduce cost; devices demonstrate reusability above 50 uses. An acrylamide monomer solution containing broad-range carrier ampholytes was microprinted with a Nano eNabler $^{\mathrm{TM}}$ between micropatterned gold electrodes spaced $300 \mu \mathrm{m}$ apart on a glass slide. The acrylamide gel was polymerized in situ followed by protein loading via printed diffusional exchange. A pH gradient formed via carrier ampholyte stacking when electrodes were energized; the gradient was verified using ratiometric $\mathrm{pH}$-sensitive FITC/TRITC dyes Green fluorescent protein (GFP) and Rphycoerythrin (R-PE) were utilized both as $\mathrm{p} /$ markers and to test sIEF performance as a function of electric field strength and ampholyte concentration. Factors hampering SIEF included cathodic drift and $\mathrm{pH}$ gradient compression, but were reduced by co-printing non-ionic Synperonic ${ }^{\circledR}$ F-108 surfactant to reduce protein-gel interactions. sIEF gels achieve protein separations in $<10$ minutes

2 The material contained in this chapter has been accepted for publication in Electrophoresis.

DOI:10.1002/elps.201600565 
yielding bands $<50 \mu \mathrm{m}$ wide with peak capacities of $\sim 8$ and minimum $\mathrm{p} /$ differences from 0.12 to 0.14 . This new sIEF technique demonstrated comparable focusing at $\sim 100$ times smaller dimensions than any previous IEF. Further, sample volumes reduced four orders of magnitude from $20 \mu \mathrm{L}$ for slab gel IEF to $0.002 \mu \mathrm{L}$ for sIEF. In summary, sIEF advantages include smaller volumes, reduced power consumption, and surface accessibility to the gel all with equivalent separation resolutions to prior IEF tools. These attributes position this new technology for rapid, in-situ protein library analysis in clinical and pharmaceutical settings.

\subsection{Introduction}

Isoelectric focusing (IEF) separates amphoteric molecules based on differences in their isoelectric points $(p / s)$. Applied DC electric fields order amphoteric species between the cathode and anode to achieve stable $\mathrm{pH}$ gradients within which molecules focus to their individual p/ [109]. Initially IEF hemoglobin and cytochrome protein separations suffered from poor resolution and $\mathrm{pH}$ gradient instabilities [141]. Carrier ampholyte (CA) mixtures, such as Bio-Lyte, Servalyt and Pharmalyte $^{\mathrm{TM}}$ [21], enabled slab gel IEF [112], capillary IEF [37], and most microfluidic chip-based IEF ( $\mu \mathrm{IEF})[4,159]$ to achieve linear, stable $\mathrm{pH}$ gradients capable of resolving proteins $\mathrm{p} /$ differences as small as $10^{-1}-10^{-2}$. With advances in microscale protein synthesis and drug conjugation in spot arrays, IEF is being displaced by more costly Edman sequencing or matrix-assisted laser desorption/ionization mass spectrometry (MALDI-MS) for peptide mass fingerprinting and post-source decay (PSD) analysis because these tools only require $\mathrm{nL}$ volumes [173].

Current slab gel IEF and cIEF require large sample volumes $(\sim 20 \mu \mathrm{L})$, high potentials ( kilovolts) and long analysis times $(\sim$ hours for slab IEF, $\sim$ tens of minutes for clEF) $[36,38]$ which are consequences of the $10-\mathrm{cm}$ and longer separation lengths. For both techniques, sample and gel preparation procedures are time and labor intensive [38]. Microfluidic chip-based IEF ( $\mu$ IEF) has enabled portability [88] while improving sample, power and time demands [4, 38, 159]; $\mu \mathrm{IEF}$ is normally conducted across $1 \mathrm{~cm}$ to $1 \mathrm{~mm}$ free solution with $\sim 0.5-1 \mu \mathrm{L}$ sample 
volumes [135, 137], $\sim 10$ s volts $[4,138,170]$, and analysis times $\sim 20$ min [126, 140]. Multiple $\mu$ IEF platforms have been demonstrated with polymer, graphene, and hydrogels channel packing or materials and microchannels, zig-zap slip chips, membrane, and $\mathrm{pH}$ layered device designs all of which have enhanced $\mu \mathrm{EFF}$ separations [174-176]. Disadvantages of free solution $\mu \mathrm{IEF}$ include discontinuous $\mathrm{pH}$ gradients caused by local potential drops and convective flow issues [88]. Thus, hybrids of slab gel IEF and free solution $\mu$ IEF were combined into polymer film coated $\mu \mathrm{IEF}$ devices $[67,148]$, microchips with thin-cut immobilized $\mathrm{pH}$ gradient (IPG) strips [127] [127], microfluidic devices with polyacrylamide gels cast inside microchannels [128, 177], followed by microchannel gels with photo-immobilized $\mathrm{pH}$ gradients $[178,179]$. These hybrid IEF techniques demonstrated stabilized $\mathrm{pH}$ gradients, sharp sample peak signals and that $\mathrm{CA}$ pH gradients effectively scale down, while separation time and reproducibility improved as size decreased [90].

Thus, we hypothesized that further miniaturization of IEF from centimeter to microscale separation lengths would offer opportunities for enhanced performance of extremely small sample volumes for rapid analysis. Since power requirements scale with separation distance, shorter gel lines require smaller applied voltages to achieve the same electric field strength. While $\mu \mathrm{IEF}$ demonstrated a 1000 -fold improvement in material consumption over slab gels. Since power requirements scale with separation distance, shorter gel lines would require smaller applied voltages to achieve comparable electric field strengths for IEF separations.

In this chapter, we demonstrates a novel surface isoelectric focusing (sIEF) technique in which ultraminiaturized gel lines of polyacrylamide monomer were printed between planar gold electrodes on a glass surface, allowed to polymerize, and then were loaded with proteins. Upon electric field application, CA alignment established the $\mathrm{pH}$ gradient, which was fluorescently imaged, and the proteins were focused to their pl points. This work further presents a parametric study of CA concentration and operating voltage to determine optimal operating conditions. Fluorescent protein samples, green fluorescent protein (GFP) and R-phycoerythrin (R-PE), were utilized to quantify and compare sIEF protein separation and focusing 67 
capabilities to literature $\mu \mathrm{IEF}$. Lastly, this work demonstrates that a non-ionic surfactant can reduce cathodic drift and $\mathrm{pH}$ gradient compression within sIEF gels. This work provides the first demonstration of surface accessible IEF, with resolutions equivalent to large-scale methods, nanogram sample volumes, voltages of $\leq 9 \mathrm{~V}$, and run times $<10$ minutes.

\subsection{Materials and Methods}

\subsubsection{Reagents}

Broad range carrier ampholytes (Pharmalyte ${ }^{\mathrm{TM}} \mathrm{pH} 3-10$ ) were purchased from GE Healthcare (Pittsburgh, PA, USA). A $40 \% \mathrm{w} / \mathrm{v}$ stock solution for acrylamide monomer was made from 29:1 ratio acrylamide and bis-acrylamide powder obtained from Sigma-Aldrich (St. Louis, MO, USA). Ammonium persulfate (APS, gel polymerization catalyst), tetramethylethylenediamine (TEMED, co-catalyst with APS for gel polymerization), and glycerol (to impede gel drying) were obtained from PlusOne (New York, NY, USA). APS at $15 \% \mathrm{w} / \mathrm{v}$ solution was used as a polymerization catalyst for the acrylamide solution. E-pure water with $18.2 \Omega \cdot \mathrm{cm}$ resistivity was made by EMD Millipore Simplicity Ultrapure 185 water system (Billerica, MA, USA). APS at $15 \% \mathrm{w} / \mathrm{v}$ solution was used as a polymerization catalyst for the acrylamide solution in E-pure water $(18.2 \Omega \cdot \mathrm{cm}$, EMD Millipore Simplicity Ultrapure 185, Billerica, MA, USA). pH sensitive Fluorescein-5isothicyanate (FITC Isomer I, Invitrogen Eugene, OR, USA), and pH insensitive TRITC (Sigma-Aldrich, St. Louis, MO, USA) dyes were pre-mixed to a 1:2 v/v ratio (50 mM FITC and $100 \mathrm{mM}$ TRITC) [180]. Green fluorescent protein (GFP, $1 \mathrm{mg} / \mathrm{mL}$, $\mathrm{p} / 6.0$, diffusion coefficient $5 \times 10^{-7} \mathrm{~cm}^{2} / \mathrm{s}$, EMD Millipore, Billerica, MA, USA) came suspended in $1 \mathrm{mM}$ phosphate buffer saline (PBS) solution. R-phycoerythrin (R$P E, 20 \mathrm{mg} / \mathrm{mL}$, p/ 4.4, diffusion coefficient $3.94 \pm 0.13 \times 10^{-7} \mathrm{~cm}^{2} / \mathrm{s}$, AnaSpec, Fremont, CA, USA) was first dialyzed using a Slide-A-Lyzer MINI Dialysis kit (10K MWCO, Sigma-Aldrich, St. Louis, MO, USA) against a 1 mM PBS (made from premixed pellets, EMD Millipore, Billerica, MA, USA) to remove extra salts, and then 
diluted to $1 \mathrm{mg} / \mathrm{mL}$ using $1 \mathrm{mM}$ PBS. Synperonic ${ }^{\circledR} \mathrm{F}-108$ surfactant (Sigma-Aldrich, St. Louis, MO, USA) was used at $1 \% \mathrm{w} / \mathrm{v}$.

\subsubsection{Device Fabrication}
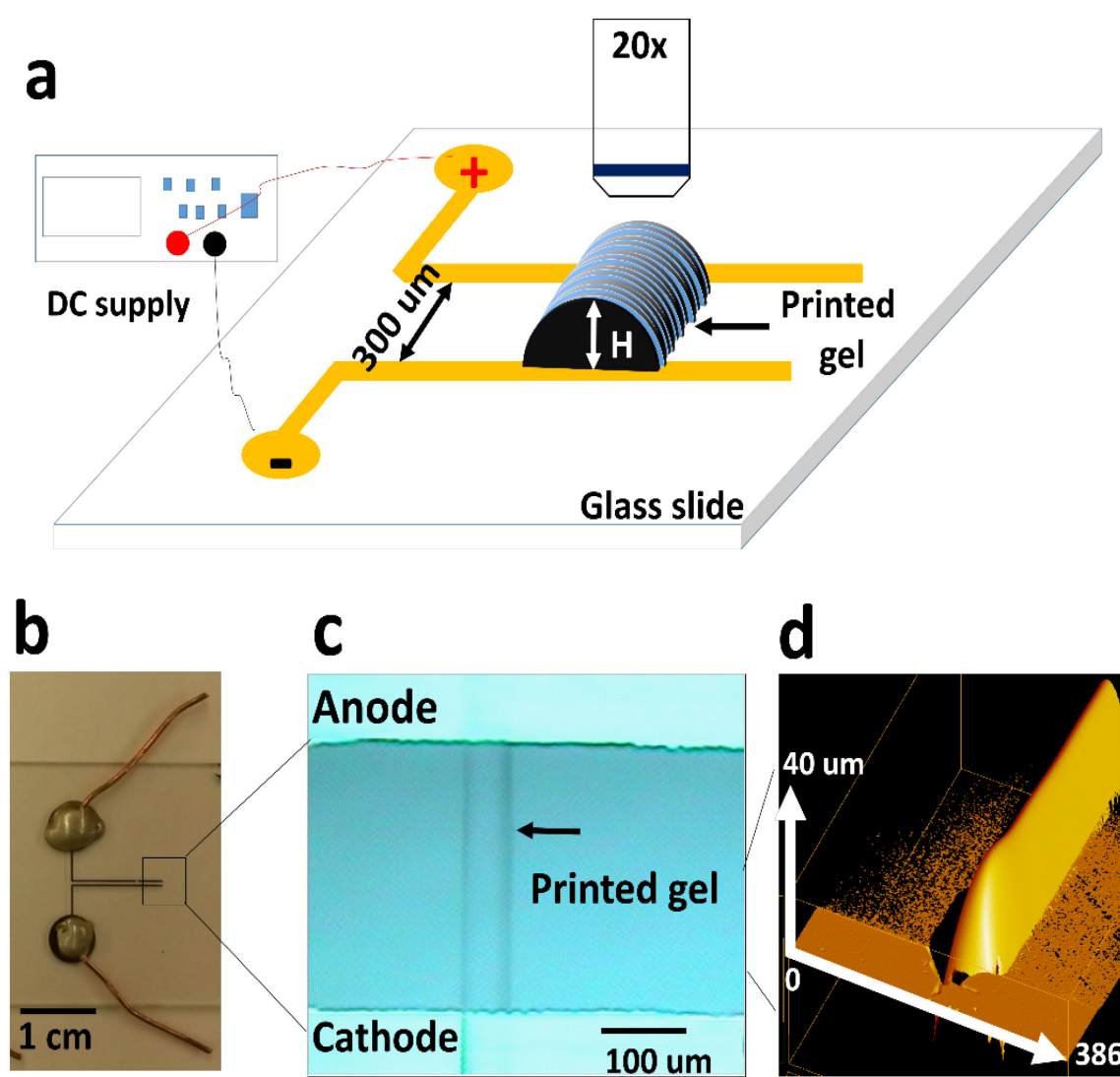

\section{c}

\section{Anode}
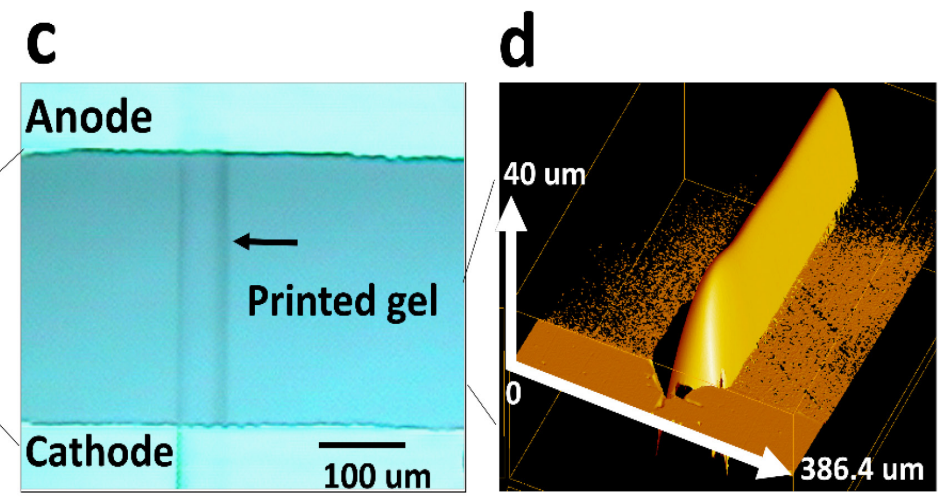

Figure 4.1 Schematic showing configuration and operation of the surfaceenabled IEF (sIEF) device. a) Schematic of sIEF device illustrating carrier ampholyte alignment into a $\mathrm{pH}$ gradient. Broad range (3-10) PharmalyteTM was co-printed with polyacrylamide between gold electrode pairs spaced $300 \mu \mathrm{m}$ apart. The gel forms in-situ; a pH gradient forms within the gel upon electric field application. The obtained gel line has half-cylindrical shape with $35 \pm 5 \mu \mathrm{m}$ height $(\mathrm{H})$. b) Photograph of sIEF device illustrating copper leads attached to micropatterned gold electrodes. c) A 20x fluorescence microscope was utilized to visualize the gel as well as the $\mathrm{pH}$ fluorophores and proteins within the gel.

A $7 \mathrm{~cm} \times 3 \mathrm{~cm}$ microscope slide was micropatterned with three sets of $100 \mu \mathrm{m}$ wide gold electrodes spaced parallel $300 \mu \mathrm{m}$ apart (Figure 4.1) prepared via soft photolithography and electro-vapor deposition. As previously demonstrated [181], 
a glass slide was spin-coated with negative photoresist (PR1-1000A, Futurrex, Franklin, NJ, USA), masked, then UV exposed (EVG620, EV-Group, Austria). Design features were developed with RD-6 developer (Futurrex, Franklin, NJ, USA), he slide was cleaned of undeveloped photoresist, then sputter-coated with $50 \mathrm{~nm}$ titanium adhesion layer followed by $150 \mathrm{~nm}$ gold (Randex sputtering system 2400, Perkin-Elmer, USA). Photoresist and excess metal were removed via acetone sonication. Copper wires were attached to the gold electrode contact pads using silver epoxy (MG chemical 8331, $0.007 \Omega \cdot \mathrm{cm}$ electrical resistivity and 0.90 $\mathrm{W} / \mathrm{m} / \mathrm{K}$ thermal conductivity).

For sIEF, a single gel line was printed via a software-programmed vector motion to the micropatterned microscope slide surface using a fluidics-enhanced molecular transfer operations (FEMTO) instrument, Bioforce Nano eNabler ${ }^{\mathrm{TM}}$ (Bioforce, USA). This tool enables precise pattern-printing of diverse fluids on arbitrary surfaces $[47,61,71-74,182]$. The instrument includes a controllable sample stage, surface patterning tool (SPT) holder, microscope with CCD camera and chamber with humidity control. The Pharmalyte ${ }^{\mathrm{TM}}$, acrylamide, and APS solutions were pre-mixed and loaded into the $60 \mu \mathrm{m}$ rounded surface patterning tool (SPT-S-C60R) mounted on the SPT holder. Acrylamide/APS solution was printed at room temperature via $100 \mathrm{~nm}$ z-dimension SPT tip resolution to the patterned glass slide on the computer controlled $x$, y sample stage $(20 \mathrm{~nm}$ resolution). A $60-\mu \mathrm{m}$-wide, 300- $\mu \mathrm{m}$-long line was printed via stage vector movements in the $x$-direction. The printed gel line overlapped the $200 \mathrm{~nm}$ thick electrodes by ca. $20 \mu \mathrm{m}$ at each end to ensure good electrical contact between the gel and the electrodes. Printing was monitored in real time and recorded at 20X magnification. Printing and polymerization parameters were optimized to achieve complete gelation (3 hours), surface properties of glass slide (10 min UV-ozone treatment to control surface hydrophobicity) and printing chamber humidity (15\% relative humidity at $25^{\circ} \mathrm{C}$ ). The $3 \mathrm{D}$ profile of the printed gel was examined and modeled via a custom-built optical profilometer. The gel surface was scanned via a laser and beam reflection was analyzed via shape modeling software. The gel 
line had a half-cylindrical shape with a height of $35+/-5 \mu \mathrm{m}$ and width (also verified via traditional optical microscope of $60 \mu \mathrm{m}$. Spent gel lines were completely removed via ethanol; the SIEF slide were cleaned via UV-ozone treatment and reused. In addition, the surface configuration enabled printing of multiple gel lines to conduct multiple IEF experiments simultaneously.

\subsection{3 pH Fluorescence Calibration and pH Gradient Quantification}

To directly image the $\mathrm{pH}$ gradient development, FITC:TRITC fluorescent dyes at $1: 2 \mathrm{v} / \mathrm{v}$ were printed over the polymerized gel lines on the sIEF slides, then allowed to stand for 5 minutes to allow fluorophore diffusion into the gel. All experiments were conducted in a darkroom and fluorescent readings were shuttered to minimize photobleaching effects. The slide was removed from the Nano eNabler ${ }^{\mathrm{TM}}$ stage and mounted on an inverted fluorescence microscope (Axiovert $200 \mathrm{M}$, Carl Zeiss, Thornwood, NY, USA) for video imaging with a 20X Neoplan objective. FITC was excited from an $\mathrm{Hg}$ light source filtered through a $485 \pm 25 \mathrm{~nm}$ band-pass filter, and emissions filtered through $515 \mathrm{~nm}$ long-pass and $535 \pm 40 \mathrm{~nm}$ band-pass filters. TRITC was excited with a $546 \pm 25 \mathrm{~nm}$ band-pass filter, and emissions filtered by a $640 \pm 25 \mathrm{~nm}$ band-pass filter. Dye/pH calibrations were performed within printed gels by co-printing FITC/TRITC with pre-made standard $\mathrm{pH} 4,5,6,7,8$, 9, and 10 solutions onto the premade gels. FITC and TRITC images were acquired every 1 minute for 10 minutes; >3 independent repeats were conducted. Fluorescent FITC and TRITC emission intensities were averaged over the gel area between electrodes with Image $\mathrm{J}$ software (NIH, USA), then normalized via equation 4.1:

$$
\mathrm{R}=\frac{\Sigma l_{i, j}^{F}}{\Sigma l_{i, j}^{T}}
$$

$R$ is the normalized intensity ratio, $I^{F}$ and $I^{T}$ are pixel intensities of FITC and TRITC, respectively, $i$ is the horizontal pixel location and $j$ is the vertical pixel location.

To image $\mathrm{pH}$ gradient formation during sIEF runs, gels were similarly prepared with FITC: TRITC and broad range Pharmalyte ${ }^{T M}$. A baseline (time 0 ) was imaged and then a $9 \mathrm{~V}$ potential was applied and recorded for 10 minutes. The 
normalized intensity ratio was translated into $\mathrm{pH}$ with the calibration curve to monitor $\mathrm{pH}$ gradient formation during sIEF runs.

\subsubsection{Isoelectric Focusing of Protein Mixture and Image Analysis}

Dialyzed GFP and R-PE samples were pre-mixed at a $1: 1 \mathrm{v} / \mathrm{v}$ ratio to achieve a final concentration of $0.5 \mu \mathrm{g} / \mu \mathrm{L}$, and then printed on top of the sIEF gel line via a SPT. Total protein loading into the micro gels were consistently $0.9 \pm 0.04 \mathrm{ng}$. This sIEF slide was mounted onto the fluorescent microscope allowing 5 minutes for protein diffusion into the gels. The uniform presence of GFP and R-PE (single channel, excitation $485 \pm 25 \mathrm{~nm}$, emission $535 \pm 40$ ) was verified at time 0 in the gel. Then, images were acquired every minute for 15 minutes to monitor protein band focusing. Experiments were repeated 3 times for each solution condition. Focused band shapes as well as intensity profiles at the gel midline were obtained for comparisons. Demonstrations of focus recording are can be seen in Figure 4.2 and 4.3. Figure 4.2 illustrates the $\mathrm{pH}$ gradient formation at $200 \mathrm{~V} / \mathrm{cm}$ electric field for 10 minutes. The first column illustrates the raw images while the second column demonstrates the image intensity profile at the midline (shown by the dashed white line in the first image). Images are shown every 2 minutes because additional granularity does not provide significant insights. Figure 4.3 illustrates the time progression of GFP and PE focusing at $200 \mathrm{~V} / \mathrm{cm}$ field electric field with Pharmalyte ${ }^{\mathrm{TM}}$ concentration of $2 \% \mathrm{w} / \mathrm{v}$ for $10 \mathrm{~min}$. Raw images are shown in the left column and the corresponding intensity profile at the midline is demonstrated in the right column. 

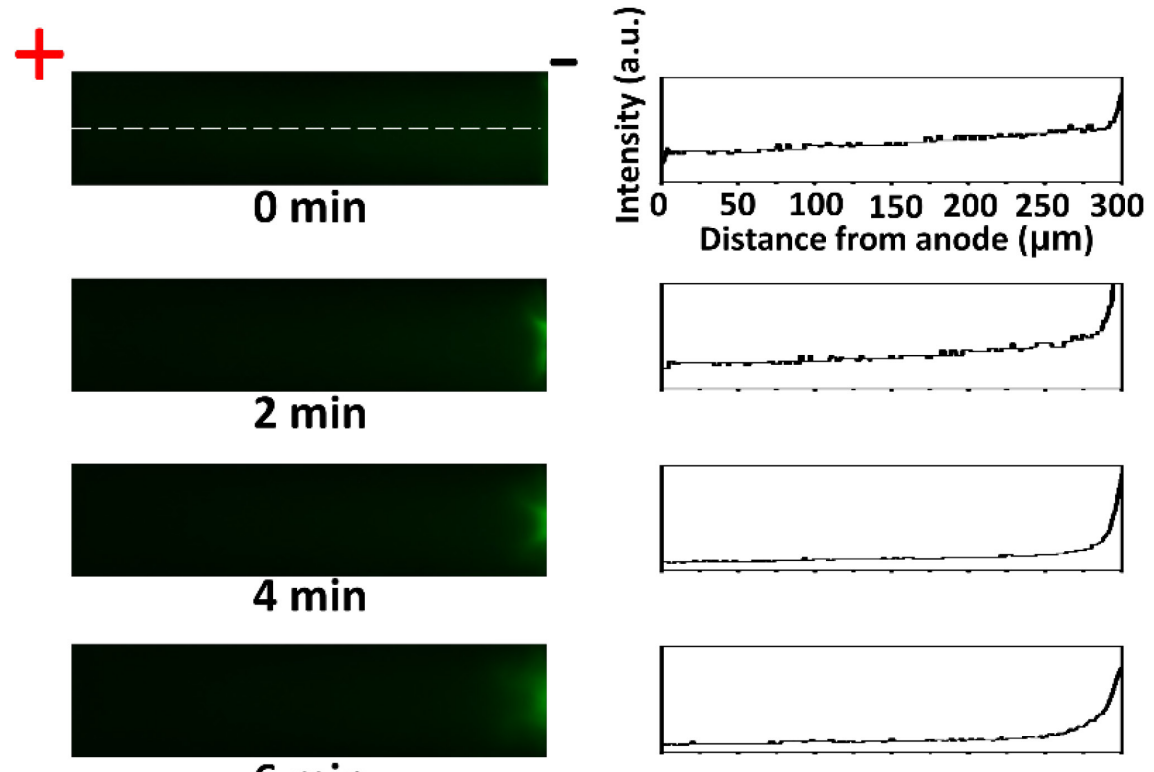

$6 \mathrm{~min}$
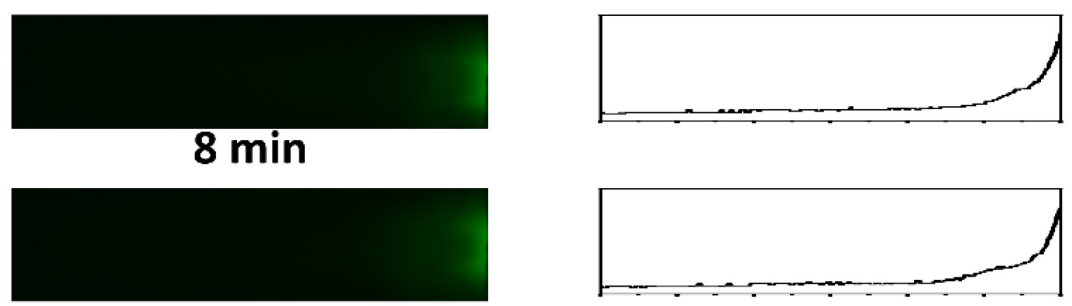

\section{$10 \mathrm{~min}$}

Figure 4.2 Images of dynamic $\mathrm{pH}$ gradient establishment within the printed polyacrylamide sIEF gels. The $50 \mu \mathrm{M}$ FITC, $100 \mu \mathrm{M}$ TRITC mixture was printed on the gels. Images shown every 2 minutes from the entire recording in the $\mathrm{pH}$ sensitive FITC channel. Intensity profile lines obtained at the midline are shown in the right column for each image. 

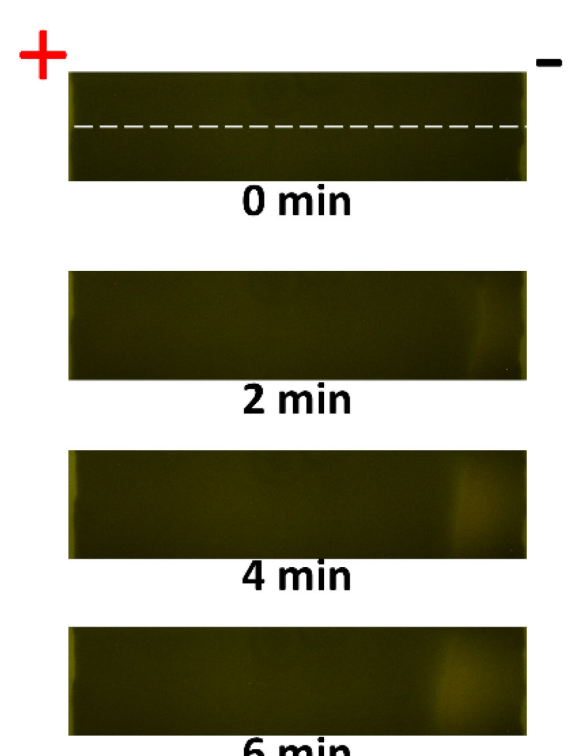

$6 \mathrm{~min}$

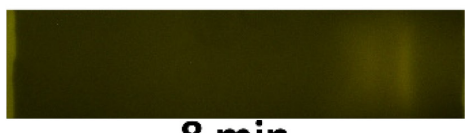

$8 \mathrm{~min}$

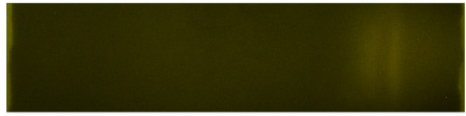

$10 \mathrm{~min}$
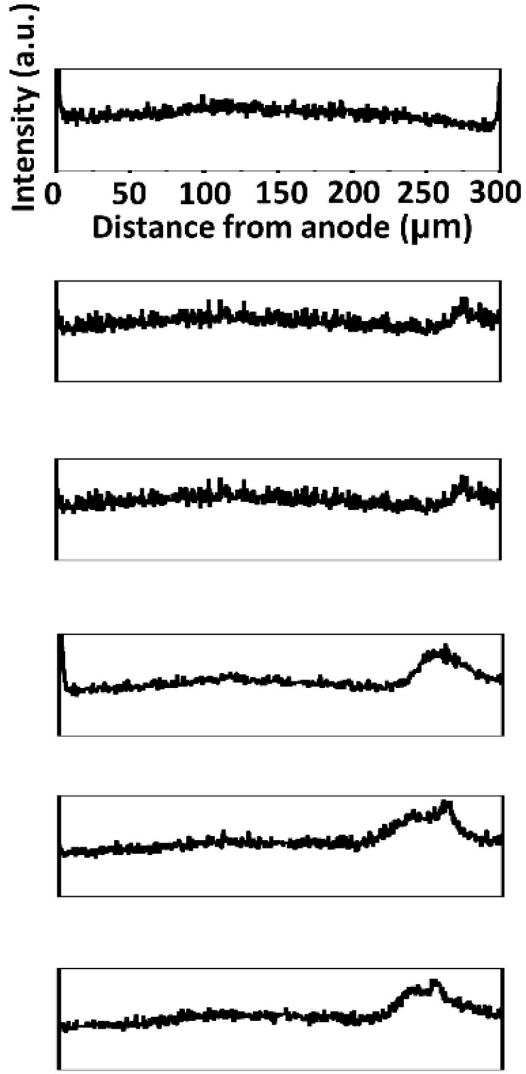

Figure 4.3 Images of dynamic GFP/R-PE protein mixture focusing within the printed polyacrylamide sIEF gels. Protein mixture contains GFP and R-PE in $1: 1 \mathrm{v} / \mathrm{v}$ ratio with $0.5 \mathrm{mg} / \mathrm{mL}$ concentration of each species. Images shown every 2 minutes for 10 minutes (images acquired every 1 minute). Intensity profile lines obtained at the midline are shown in the right column for each image.

\subsection{5 sIEF Dependencies and Optimization}

Electric field strength and carrier ampholyte concentration dependencies were investigated. DC potentials from 3 to $9 \mathrm{~V}$ were applied across the $300 \mu \mathrm{m}$ gel line to achieve electric fields from $100 \mathrm{~V} / \mathrm{cm}$ to $300 \mathrm{~V} / \mathrm{cm}$. CAs concentrations of $2 \%$, $4 \%$ and $6 \% \mathrm{w} / \mathrm{v}$ Pharmalyte ${ }^{\mathrm{TM}}$ were examined under experimental conditions described in section 2.5. To explore sIEF optimizations, $1 \% \mathrm{w} / \mathrm{v}$ Synperonic ${ }^{\circledR} \mathrm{F}-108$ surfactant, CHAPS (zwitterionic detergent, Sigma-Aldrich), or Dithiothreitol (DTT, 
Sigma-Aldrich) were added into the acrylamide/APS solution (section 2.1), with $2 \%$ w/v Pharmalyte ${ }^{\mathrm{TM}}$ and 6V DC was applied. Pre-mixed GFP and R-PE were used at a $1: 1 \mathrm{v} / \mathrm{v}$ ratio.

\subsubsection{Quantitative sIEF Resolution Power Estimations}

The focusing effectiveness of SIEF was determined, similar to other IEF platforms $[91,183]$, by quantifying the minimum difference between isoelectric points, $\Delta p /$, and peak capacity, $n$ (see Equation 4.2, 4.3 and 4.4). These parameters indicate resolvability of proteins by $\mathrm{p} /$ as well as the number of protein species that can be distinguished within an IEF gel, respectively $[91,183]$. For $\Delta p /$, the $\mathrm{pH}$ gradient, $\mathrm{d}(\mathrm{pH}) / \mathrm{dx}$, diffusion coefficient, $\mathrm{D}$, and $\mathrm{pH}$ gradient mobility difference, $-\mathrm{d} \mu / \mathrm{d}(\mathrm{pH})$, are assumed constant under ideal conditions [102]. Most commercially available CAs achieve reproducible and stable $\mathrm{pH}$ gradients in PAGE gels such that these assumptions are valid, at least over shorter operation times before cathodic drift and compression issues compound. Thus, $\Delta \mathrm{p} /$ is directly tunable via electric field strength, E [102]:

$$
\Delta \mathrm{p} I_{\min }=3 \sqrt{\frac{\mathrm{D} / \mathrm{E}}{\left(\frac{\mathrm{dpH}}{\mathrm{dx}}\right) /\left(-\frac{\mathrm{d \mu}}{\mathrm{dpH}}\right)}}
$$

To calculate $\Delta \mathrm{p} /$ from experiments, $\mathrm{pH}$ gradients were determined directly from the image, diffusion coefficients were specific for R-PE and GFP, and the mobility difference was approximated as 1 from Pharmalyte ${ }^{T M}$ titration curves. The theoretical expression for peak capacity, $\mathrm{n}_{\mathrm{t}}$ adds additional insights into separation performance. Under ideal conditions, the $\mathrm{pH}$ gradient and effective charge over $\mathrm{pH}, \mathrm{dq} / \mathrm{d}(\mathrm{pH})$, are considered constant and $\mathrm{n}_{\mathrm{t}}$ is directly tunable via $\mathrm{E}$ [38],

$$
\mathrm{n}_{\mathrm{t}}=\sqrt{\frac{\left.-\mathrm{FE}\left[\frac{\mathrm{dq}}{\mathrm{d}(\mathrm{pH})}\right] \mathrm{I} \frac{\mathrm{d}(\mathrm{pH})}{\mathrm{dx}}\right] \mathrm{L}^{2}}{16 \mathrm{RT}}}
$$

Same to Equation 2.3 in Chapter 2, F is Faraday's constant, $\mathrm{R}$ is the gas constant, $T$ is the temperature, and $L$ is the total separation length. To calculate peak 
capacity, $\mathrm{n}_{\mathrm{e}}$, from experiments, we adapt, as others have done, a method from capillary IEF and chromatography to the sIEF intensity profiles as shown in Equation 4.4 [106-108],

$$
n_{e}=1+\frac{L}{w}
$$

Where $w$ is the peak width taken at $4 \sigma$ where $\sigma$ is the standard deviation of the intensity peak. This $4 \sigma$ is consistent with equation 4 , which expresses this as the square root of 16 . The minimum difference between isoelectric points and peak capacities are compared across experimental conditions.

\subsection{Results and Discussion}

IEF miniaturization onto surfaces is motivated by in situ protein analysis applications due to the need to increase protein array versatility, enable rapid analysis, and reduce materials and resource costs. Given that power requirements scale with separation distance, shorter gel lines require smaller applied voltages to achieve the same electric field strength. Since $1 \mathrm{~mm} \mu \mathrm{IEF}$ demonstrated a 1000fold improvement in material consumption over 15 20 cm slab gels, a 100-1000 fold improvement in sample consumption and reduced power requirements is possible when IEF is scaled to $300 \mu \mathrm{m}$ in length as detailed herein. This section details results from this first sIEF evaluation including direct $\mathrm{pH}$ gradient imaging with the sIEF gels, electric field strength and carrier ampholyte concentration dependencies, followed by band focusing optimizations via surfactant additions.

\subsection{1 $\mathrm{pH}$ Calibration and $\mathrm{pH}$ Gradient Imaging}

Printed gel lines were half-cylindrical in shape with heights of $35 \pm 5 \mu \mathrm{m}$ and widths (also verified via traditional optical microscope) of $60 \pm 5 \mu \mathrm{m}$. To dynamically observe and characterize the formation of a $\mathrm{pH}$ gradient within the gel line, a ratiometric FITC/TRITC calibration was first performed followed by sIEF $\mathrm{pH}$ gradient imaging. For the calibration, molecularly similar and identically charged FITC, a pH-dependent fluorophore, and TRITC, a pH insensitive fluorophore, were measured ratiometrically with standard $\mathrm{pH}$ solutions in the gel lines as described 
in materials section, Equation 4.1 to yield Figure 4.2 calibration results. An exponential fit to the calibration curve is consistent with prior fluorophore results [180], as shown in the following equation and in Figure 4.2:

$$
\mathrm{pH}=8.76 \ln (\mathrm{R})+1.67
$$

Where $\mathrm{R}$ is the FITC/TRITC intensity ratio from Equation 9. Experimental data and the fitted curve match well between $\mathrm{pH} 4$ to 9 nearly covering the Pharmalyte ${ }^{\mathrm{TM}} \mathrm{pH}$ 3-10 range. For sIEF $\mathrm{pH}$ gradient imaging, $\mathrm{pH}$ was ratiometrically quantified during electric field application. The fluorescent image in Figure $3 a$ demonstrates the $\mathrm{pH}$ gradient profile with a plateau region 0-210 $\mu \mathrm{m}$ from the anode and an increasing $\mathrm{pH}$ gradient from 210 to $300 \mu \mathrm{m}$. Using the calibration curve in Figure 4.2, the corresponding $\mathrm{pH}$ intensity profile is calculated and shown in Figure 4.3b, right hand axis.

a

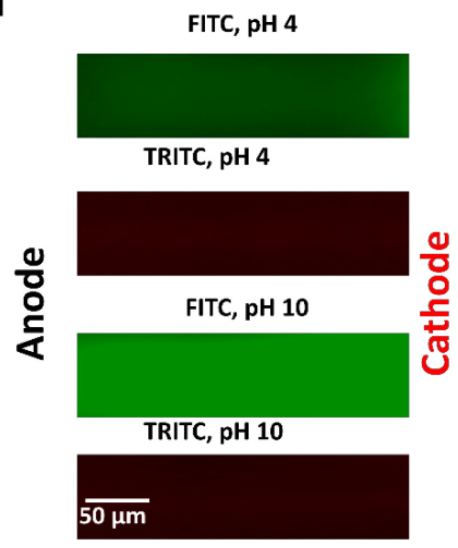

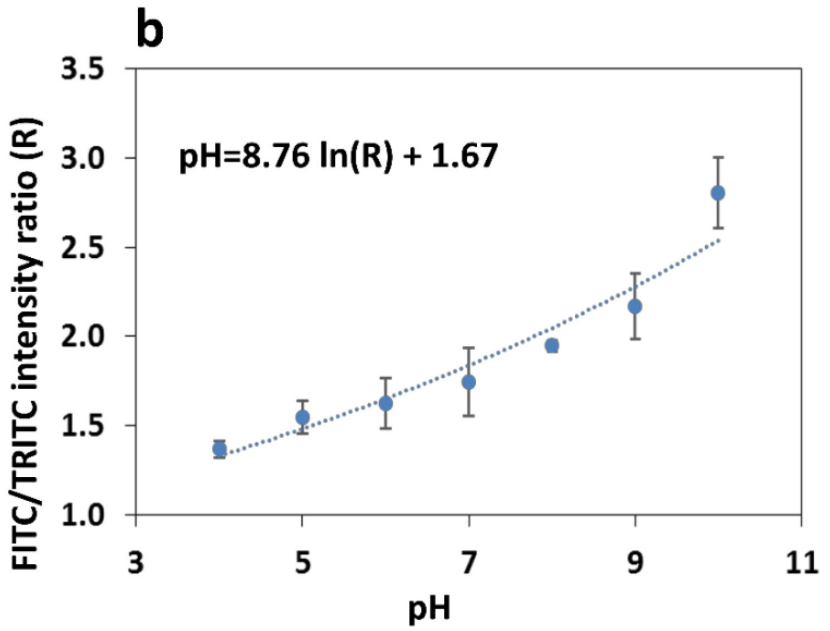

Figure 4.4 Calibration of $\mathrm{pH}$ fluorophores within the polyacrylamide gels. a) Fluorescent images of the surface printed polyacryalamide gel lines adjusted to $\mathrm{pH} 4$ and $\mathrm{pH}$ 10. The $50 \mu \mathrm{M}$ FITC, $100 \mu \mathrm{M}$ TRITC mixture at a $1: 2 \mathrm{v} / \mathrm{v}$ ratio was printed on top of the polymerized gels. Nearly simultaneous 20x microscope images were obtained with FITC $535 \pm 40 \mathrm{~nm}$ and TRITC $640 \pm 25 \mathrm{~nm}$ bandpass filters. b) Intensity data from FITC images were normalized by TRITC images, then fit to a curve with the trend line equation shown. Error bars are standard error for $n=3$ repeats. 
Ideally, commercial carrier ampholytes should generate a linear $\mathrm{pH}$ gradient that spans the anode to cathode gel length of $300 \mu \mathrm{m}$. Figure 4.3 illustrates the $\mathrm{pH} 3-$ 10 CAs were compressed near the cathode, a common phenomenon reported previously [91, 96, 159, 184-187] In a large slab gel with small reservoirs that allowed electrolytes to diffuse into the gel, the $\mathrm{pH}$ gradient was observed to compress due to differing anion and cation migration rates within the gel lines [162]. Similarly, a free solution $\mu$ IEF device with reservoirs at either end of a channel and similar electrolyte diffusion yielded compression [4]. In our sIEF configuration, the separation channel was micrometers in length and no reservoirs were utilized with the intent to minimize anion/cation disruptions. However, the compression observed in Figure 4.3 suggests $\mathrm{pH}$-disruptive ion migration effects remain, likely due to Faradaic reactions at the electrode surface [181]

\subsubsection{Demonstration of Protein Focusing in SIEF}

Despite $\mathrm{pH}$ gradient compression issues, trial protein IEF runs were conducted at $2 \% \mathrm{w} / \mathrm{V}$ Pharmalyte ${ }^{\mathrm{TM}}$ concentration and $200 \mathrm{~V} / \mathrm{cm}$ applied electric field strength. A 1:1 mixture of fluorescent GFP and R-PE proteins, were printed onto the micro gels and monitored during electric field focusing. Figure 4.3a gel images illustrate that R-PE was separated from GFP and focused into two straight bands at positions of $225 \mu \mathrm{m}$ and $260 \mu \mathrm{m}$ from the anode, respectively. Protein peak locations were compared against the $\mathrm{pH}$ gradient intensity profile in Figure $4.3 \mathrm{a}$ and $\mathrm{b}$. At the R-PE band position $225 \mu \mathrm{m}$ from the anode, the corresponding calibration-inferred $\mathrm{pH}$ value is $\sim 4$, while the $\mathrm{pH}$ reading at the GFP band position of $260 \mu \mathrm{m}$ is $\sim 6$. These two values are close to the known isoelectric points of RPE $(p /=4.4)$ and GFP $(p /=6.0)$. The consistency between protein IEF and the established $\mathrm{pH}$ gradient illustrate that protein migration and isoelectric focusing to 
bands can be successfully achieved in microprinted acrylamide gel lines at length scales almost 100 times smaller than previous $\mu$ IEF.

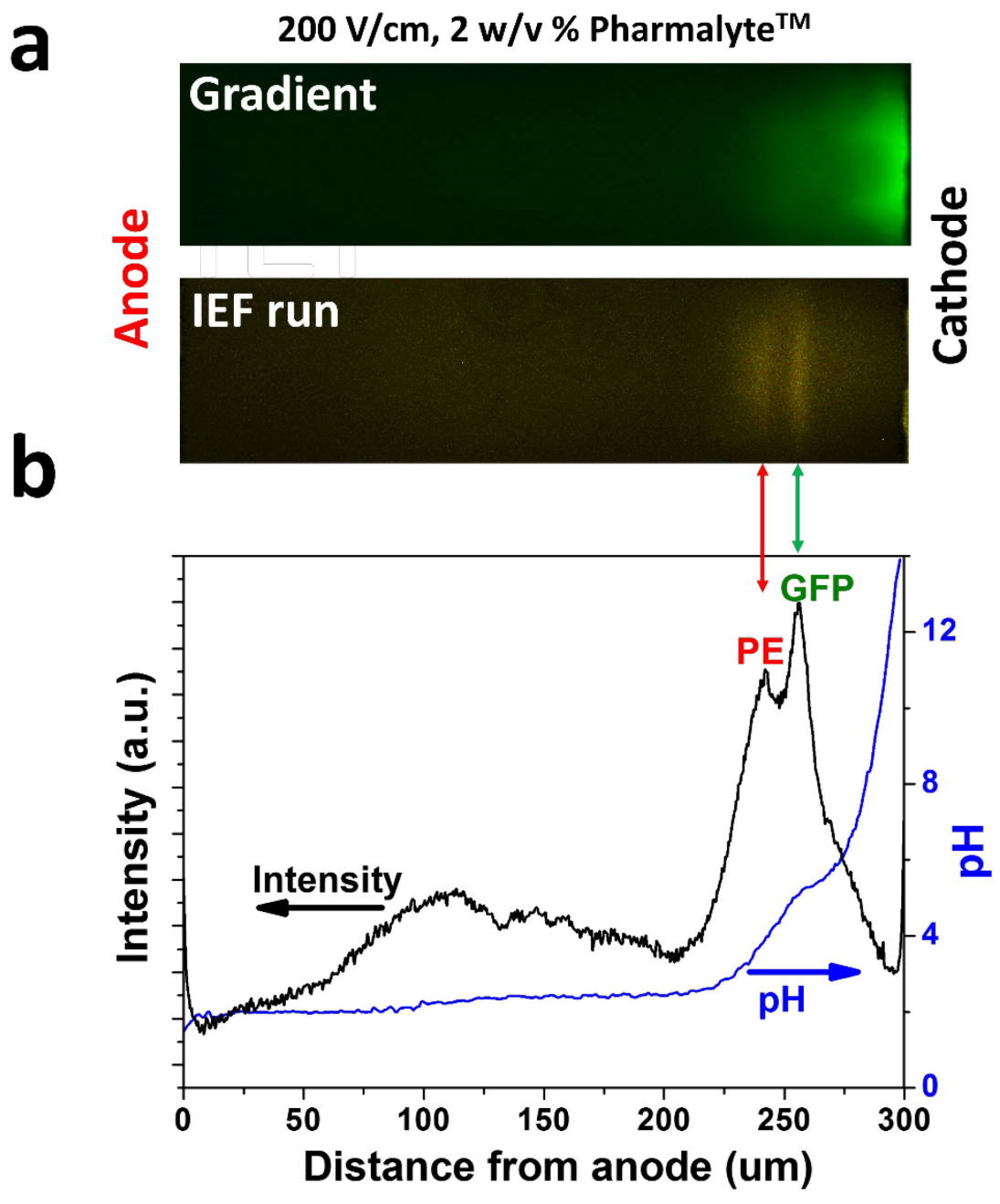

Figure $4.5 \mathrm{pH}$ gradient establishment and protein focusing in sIEF. a) Direct imaging of the $\mathrm{pH}$ gradient established within the printed polyacrylamide sIEF gels. b) Demonstration of SIEF separation of Rphycoerythrin (R-PE, $\mathrm{pl}=4.4$ ) from green fluorescent protein (GFP, $\mathrm{pl}=6.0$ ) within a $200 \mathrm{~V} / \mathrm{cm}$ field and $2 \%$ w/v Pharmalyte ${ }^{\mathrm{TM}}$ to gel. b) Corresponding intensity profiles for the $\mathrm{pH}$ gradient (blue, right hand side y-axis) and for R-PE, GFP sIEF separation (black, left side y-axis) as a function of position. 


\subsubsection{Electric Field Strength Optimization}

As suggested by Equations 4.2, the factors we examined to optimize sIEF operation included electric field strength and carrier ampholyte concentration because these directly influence peak width and resolvability as well as the $\mathrm{pH}$ gradient. The impact of electric field strength $(100,200$, and $300 \mathrm{~V} / \mathrm{cm})$ on band focusing was investigated and quantified via $\Delta \mathrm{p} /$ and $\mathrm{n}$. Figure 4.4 demonstrates typical images of the separated and focused proteins along with their corresponding intensity profiles between the anode and cathode. To compare focusing potential intensity profiles were obtained at the centerline of the gel thus neglecting gel edge effects. Figure 4.4a illustrates both protein species focused at all three electrical field strengths after 10 minutes while Figure $4.4 \mathrm{c}$ illustrates protein focusing at $3 \mathrm{~min}$ for $300 \mathrm{~V} / \mathrm{cm}, 5 \mathrm{~min}$ for $200 \mathrm{~V} / \mathrm{cm}$. Individual protein focusing efficiency, band shape, and position in the gels differ. Total protein in the gel was fixed and thus larger intensities per pixel area in Figures $4 \mathrm{~b}$ and d indicate more effective focusing. Total protein in the gel was fixed and thus larger intensities per pixel area indicate more effective focusing. As illustrated in Figure 4.4, protein focusing efficiency increases with increasing field strength. This is expected because higher electric fields impart greater electrophoretic forces to overcome any protein/gel interactions impeding protein mobility.

However, $100 \mathrm{~V} / \mathrm{cm}$ and $300 \mathrm{~V} / \mathrm{cm}$ gel bands displayed distortion at the gel's side edges, whereas $200 \mathrm{~V} / \mathrm{cm}$ produced smooth smiling bands for GFP and a weaker undistorted straight band for R-PE as shown in Figure 4.4a. Distortion in smaller electric fields, such as $100 \mathrm{~V} / \mathrm{cm}$, are more susceptible to gel non-uniformity effects because protein migration is not strong enough to overcome pore geometry differences [188]. These effects become more pronounced at walls, or in our system, the gel side edges, because the field is weaker and the interface hinders protein transport pathways. With increasing electric field strength $(200 \mathrm{~V} / \mathrm{cm})$, the IEF driving force is able to overcome gel nonuniformities and geometric limitations, thus yielding smoother bands. Further increases in field strength $(300 \mathrm{~V} / \mathrm{cm})$ increase the tendency and strength of electrokinetic and hydrodynamic effects 
[159]. For the band shape illustrated at $300 \mathrm{~V} / \mathrm{cm}$, electric double layer induced electroosmotic flows (EOF) could be occurring at both the gel/glass interface as well as the gel/air interface. The gel is fully saturated with sample and CA solution, so it is possible a thin liquid film exists as well. In summary, band shape dependencies are complex depending upon gel uniformities and competing electroosmosis and hydrodynamic effects within the narrowing edges of the gel. Thus, all future experiments were conducted at $200 \mathrm{~V} / \mathrm{cm}$ because protein transport effects were consistently more uniform across all repeats.

Band position, measured as distance from the anode, varied with electric field strength as shown in Figure 4.4a. Idealy, the $\mathrm{pH}$ gradient should be liner across the entire separation length. In this case, $\mathrm{pH}$ gradient of 4.4-6 should have length of $47 \mu \mathrm{m}$ (band position distance between PE $134 \mu \mathrm{m}$ and GFP $181 \mu \mathrm{m}$ from anode). In the real experiment, band positions directly correlate with the level of $\mathrm{pH}$ gradient compression and drift, the closer bands' distance from anode, the less cathodic drift; the larger distance between bands, the less the $\mathrm{pH}$ gradient compression experienced. At $100 \mathrm{~V} / \mathrm{cm}$ electric field, GFP and PE band are 250 $\mu \mathrm{m}$ and $260 \mu \mathrm{m}$ from anode, respectively, with bands distance of $10 \mu \mathrm{m}$. . When the electric field increased to $200 \mathrm{~V} / \mathrm{cm}$, GFP band slightly moved by $5 \mu \mathrm{m}$ closer to anode, and PE moved by $2 \mu \mathrm{m}$, the distance between two bands increased to $13 \mu \mathrm{m}$. The higher electric field improved $\mathrm{pH}$ compression from $79 \%$ to $72 \%$ and reduced cathodic drift. At $300 / \mathrm{cm}$, both R-PE and GFP shifted $10 \mu \mathrm{m}$ closer to the anode, but band spacing did not change suggesting a reduction in cathodic drift but no improvement in $\mathrm{pH}$ gradient compression. Reducing cathodic drift and $\mathrm{pH}$ gradient compression was desired, so $200 \mathrm{~V} / \mathrm{cm}$ was identified as the optimal electric field strength. 

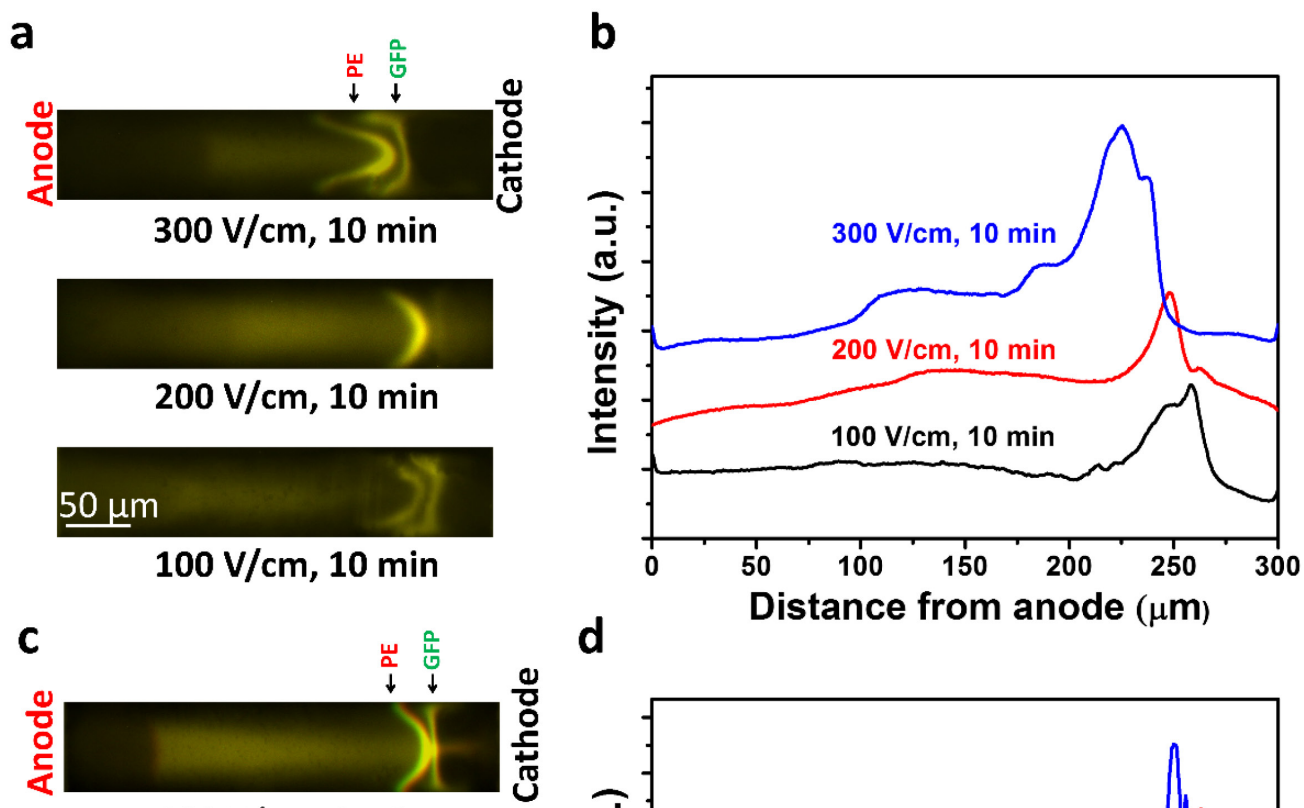

d

$300 \mathrm{~V} / \mathrm{cm}, 3 \mathrm{~min}$

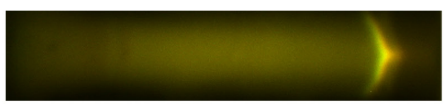

$200 \mathrm{~V} / \mathrm{cm}, 5 \mathrm{~min}$

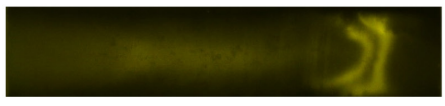

$100 \mathrm{~V} / \mathrm{cm}, 10 \mathrm{~min}$

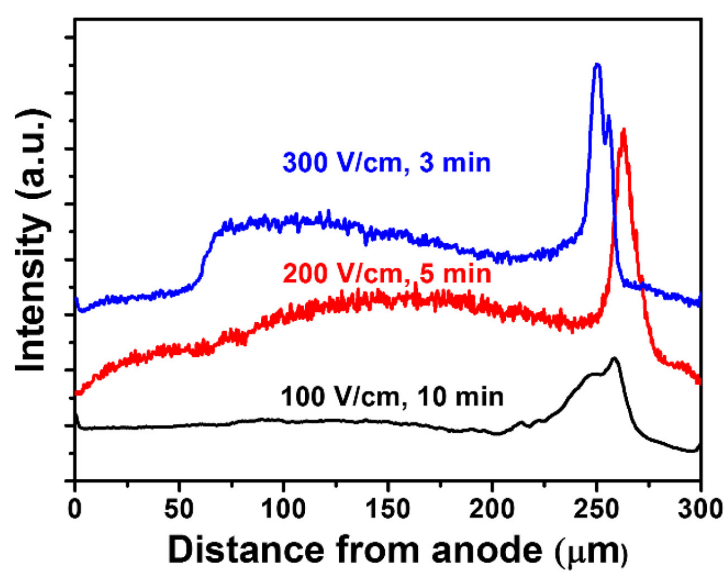

Figure 4.6 Electric field comparisons of GFP and R-PE protein separations in sIEF gels after 10 minutes in $300 \mathrm{~V} / \mathrm{cm}, 200 \mathrm{~V} / \mathrm{cm}$, and $100 \mathrm{~V} / \mathrm{cm}$. Pharmalyte was co-printed at $4 \% \mathrm{w} / \mathrm{v}$ with the polyacrylamide gel. For better visualization, images were enhanced via $+5 \%$ brightness and $+10 \%$ contrast. a) Fluorescent images of the gels with the GFP and R-PE bands illustrating shape and focusing efficiency differences. b) Corresponding protein intensity profiles illustrate band positions shift toward the cathode with decreasing voltage and protein focusing efficiencies increase with greater voltages. More uniform and preferred protein band shapes were consistently obtained at $200 \mathrm{~V} / \mathrm{cm}$. 


\subsubsection{Carrier Ampholyte Concentration Optimization}

Carrier ampholyte (CA) concentrations were also systematically varied to ascertain the most stable, uncompressed $\mathrm{pH}$ gradient between the anode and cathode. Ampholyte species are essential for stable $\mathrm{pH}$ gradient formation and also strongly influence protein behavior during IEF. Prior CA concentrations were reported in the $2-4 \% \mathrm{w} / \mathrm{v}$ range [21]. Due to the size of the gel in our sIEF device and the absence of reservoirs at either end, we expanded this range up to $6 \% \mathrm{w} / \mathrm{v}$ to determine if Pharmalyte $^{\mathrm{TM}}$ concentration may have a greater influence. Fluorescent images and corresponding intensity profiles for three CA concentrations $(2 \%, 4 \%$, and $6 \% \mathrm{w} / \mathrm{v})$ at $200 \mathrm{~V} / \mathrm{cm}$ are shown in Figure 4.5 . Images illustrate that R-PE and GFP proteins separation and focusing at all three Pharmalyte ${ }^{\mathrm{TM}}$ concentrations meaning that stable $\mathrm{pH}$ gradients formwith all $2 \%-6 \%$ w/v CA concentrations. However, protein migration behavior differed among the Pharmalyte ${ }^{\mathrm{TM}}$ concentrations: in $4 \%$ and $6 \% \mathrm{w} / \mathrm{v}$, a curved R-PE band and a straight GFP band were observed although protein focusing efficiency was greater at $4 \% \mathrm{w} / \mathrm{v}$ for R-PE and slightly greater at $6 \% \mathrm{w} / \mathrm{v}$ for GFP. In the $2 \% \mathrm{w} / \mathrm{v}$ case, RPE and GFP bands focused into straight lines, although efficiency was reduced as evidenced by lower band intensities. To track protein focusing progress currenttime plots were tracked for each run as shown in Figure $4.5 \mathrm{~b}$ inset. Current dropped then stabilized to $\sim 20 \mathrm{nA}$ as CAs and proteins approach electroneutrality upon focusing to their pl. Thus, a low stable current was an indicator of a fully developed $\mathrm{pH}$ gradient. The $2 \% \mathrm{w} / \mathrm{v}$ current trace suggests rapid alignment and focusing within ca. 2.5 minutes while both $4 \%$ and $6 \% \mathrm{w} / \mathrm{v}$ concentrations exhibit a shoulder near ca. $2.5 \mathrm{~min}$ and delayed stabilization until $5 \mathrm{~min}$ or longer. The straight bands in $2 \% \mathrm{w} / \mathrm{v}$ correlate with more rapid focusing and stabilization, whereas the curved bands and dispersed, unfocused proteins contribute to the delays in $4 \%$ and $6 \% \mathrm{w} / \mathrm{v}$ Pharmalyte ${ }^{\mathrm{TM}}$ gels. These observations are consistent with prior reports that CAs concentrations influence IEF focusing behaviors [189]. Concentrations up to $32 \% \mathrm{w} / \mathrm{v}$ revealed that higher CAs concentrations take longer to establish stable $\mathrm{pH}$ gradients and ampholyte concentrations effect protein 
behavior during IEF [190]. At low CAs concentration (2 4\% w/v), ampholyte mobility was not effected by ion strengths. However, at higher CA concentrations, free ions above $56.1 \mathrm{mM}$ change the transient states of CAs and proteins. Our sIEF gels have a total volume of $0.9 \mathrm{~nL}$, and cross sectional area of $\sim 1400 \mu \mathrm{m}^{2}$ which suggests ions present have a greater probability of interacting with the carrier ampholytes as they pack into localized regions in the gel. These interactions are less probable at lower CA concentrations, likely explaining why the $2 \% \mathrm{w} / \mathrm{v}$ results were more consistently reliable than $4 \% \mathrm{w} / \mathrm{v}$ CA. Thus, to successfully form a pH gradient and minimize ion effects, the $2 \% \mathrm{w} / \mathrm{v}$ CA concentration was selected as an optimal value for subsequent sIEF experiments.

\subsubsection{Additional Improvements for SIEF}

These preliminary results demonstrate the viability and potential for dynamically printable surface isoelectric focusing. Inspired by prior IEF knowledge, mechanisms impeding protein focusing as well as practical approaches to improve separation performance are briefly discussed. First, $\mathrm{pH}$ gradient compression has been attributed to $\mathrm{pH}$-disruptive anion and cation accumulation near the electrodes $[155,164]$ either from free ion sources or electrochemical reaction byproducts. In sIEF, Faradaic reaction byproducts can alter glass-gel interfaces and/or cause adverse protein-protein interactions. Byproducts can, in severe cases, cause gel degradation [150,152, 191]. Additional influencers of free ion concentrations include glycerol, gel components, and/or protein solution impurities. As discussed in sample preparation section, dialysis was utilized to reduce free ion concentrations and glycerol was employed to impede sample drying. Regardless of the source, higher concentrations of ions in the gel adversely affect band focusing behaviors $[138,159]$. Second, electroosmotic flows at interfaces may exacerbate band distortion [192, 193], and can also cause protein precipitation due to removal of the water hydration layer around proteins [193]. Some protein precipitation and stagnation within the gels were observed as protein residues near the anode in our sIEF experiments apparent in Figure 4.5a, 6\% w/v. Last, band distortion is sometimes attributed to Joule heating and spatial variations in 
temperature [194]. However, due to the large glass surface area under the sIEF gel lines and glass thermal conductivity, calculations show this would be less than $0.5^{\circ} \mathrm{C}$. Thus, this work explores additives to counter negative ion effects.
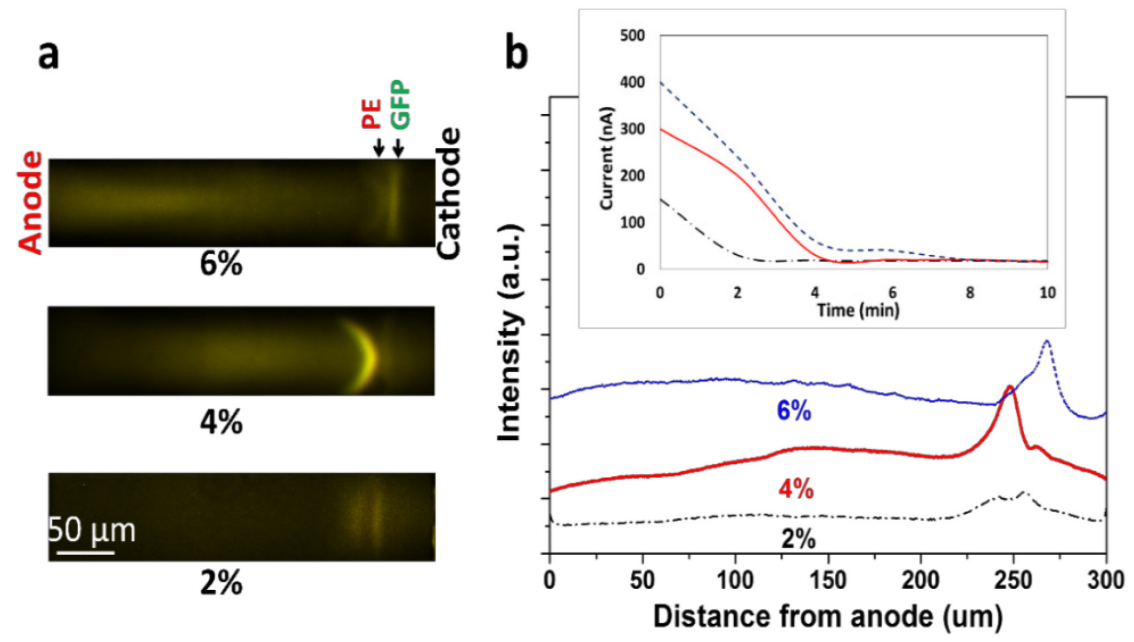

Figure 4.7 Pharmalyte ${ }^{T M}$ concentration dependence on sIEF gelbased GFP and R-PE protein separations for $6 \% \mathrm{w} / \mathrm{v}, 4 \% \mathrm{w} / \mathrm{v}$ and $2 \% \mathrm{w} / \mathrm{v}$. A $200 \mathrm{~V} / \mathrm{cm}$ electric field was applied for $10 \mathrm{~min}$. For better visualization, all the images are enhanced by $5 \%$ brightness and $10 \%$ contrast. a) Fluorescent images illustrate the shape and intensity differences between GFP and R-PE bands for the different Pharmalyte ${ }^{\mathrm{TM}}$ concentrations. b) Corresponding intensity profiles illustrate different relative protein capture efficiencies with only minor band position changes. Inset for $b$ ) is the current-time plot for each separation shown. A $2 \% \mathrm{w} / \mathrm{v}$ Pharmalyte ${ }^{\mathrm{TM}}$ concentration best facilitated protein focusing into two straight bands within the shortest time. 
To reduce protein/gel interactions as well as protein/surface interactions, a nonionic surfactant was added for the sIEF runs. In these experiments, $1 \% \mathrm{w} / \mathrm{v}$ of Synperonic $^{\circledR}$ F-108 surfactant was added to the acrylamide/APS solution (materials section), then sIEF was run exactly like previous trials. Figure 4.6 illustrates improved protein focusing efficiency and reduced $\mathrm{pH}$ compression with the $1 \%$ w/v F-108. Compared with the fluorescent image in Figure 4.3, the GFP and R-PE bands show an obvious focusing roughly halfway between the anode

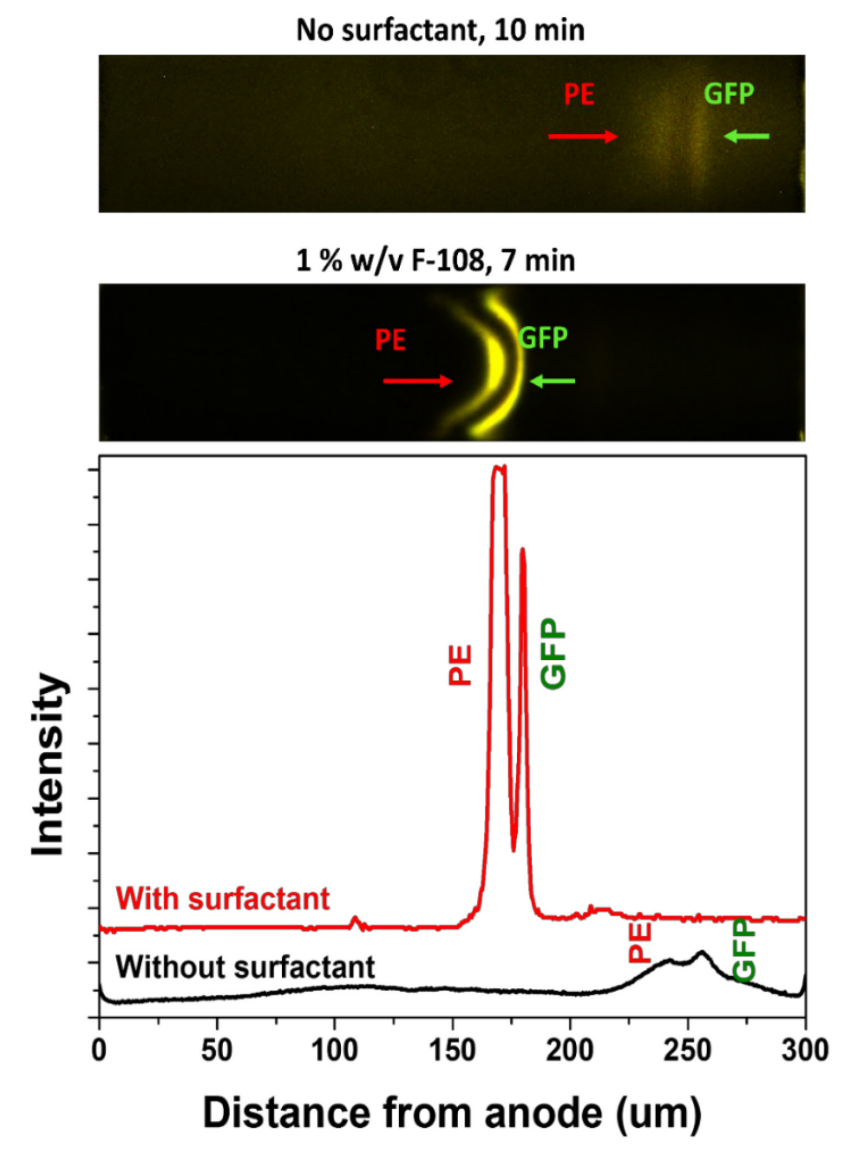

Figure 4.8 Demonstration of surfactant improved focusing efficiency. Both microscope images and corresponding intensity profiles are include in the figure. After F-108 surfactant introduced, intensified bands as well as suppressed gradient drift can be seen from microscope images, and those effect can be verified by shaped peaks and moved peak locations in intensity profiles. Also the focusing time was slightly reduced. Experiments run under the conditions of $200 \mathrm{~V} / \mathrm{cm}$ and $2 \% \mathrm{w} / \mathrm{v}$ Pharmalyte $^{\mathrm{TM}}$. 
and cathode meaning $\mathrm{pH}$ gradient compression was substantially reduced. In addition, the bands were focused with high intensity, which implies greater protein capture capability to their isoelectric point, without noticeable protein residues elsewhere in the gel. Lastly, bands exhibited the traditional crescent curvature without the severe band distortions observed in Figure 4. Using a surfactant to coat capillary surfaces, prior cIEF results showed EOF can be reduced while increasing the local viscosity near the surface $[148,149,195]$. Our results illustrate that gel co-printing with surfactant F-108 may may similarly reduce EOF and protein/gel/surface interactions.

\subsection{6 sIEF Resolving Power}

From conventional IEF theory, separation performance can be estimated using the minimum $\mathrm{p} /$ difference and peak capacity. The minimum $\mathrm{p} /$ difference, $\Delta \mathrm{p} /$, was calculated by applying Equation 1. Table 4 compares the smallest $\mathrm{p} /$ difference that can be resolved for GFP and R-PE peaks in gels with and without the nonionic surfactant. The $\Delta p /$ values of GFP and R-PE without surfactant are 0.14 and 0.12 , but with surfactant this reduces to 0.05 and 0.09 , respectively. The magnitude of these sIEF results are similar to and slightly smaller than both CIEF $\left(1 \times 10^{-1}\right)$ and free-flow/microchip IEF $\left(2\right.$ to $\left.4 \times 10^{-1}\right)$ [91, 137, 196, 197].

Table 4 Comparison of experimental peak capacity and minimal pl difference from surfactant trial and non-surfactant trial

\begin{tabular}{|l|l|l|}
\hline Peak and Condition & Peak Capacity & $\Delta \mathrm{p} /$ \\
\hline GFP, without surfactant & 8 & 0.14 \\
\hline GFP, with surfactant & 24 & 0.05 \\
\hline R-PE, without surfactant & 8 & 0.12 \\
\hline R-PE, with surfactant & 53 & 0.09 \\
\hline
\end{tabular}

Theoretical and experimental peak capacities were estimated and compared from equations (3) and (4). For both GFP and R-PE without surfactant, $n_{e}$ are $\sim 8$ while the theoretical $\mathrm{n}_{t}$ values for GFP and R-PE were both around 10. Experimental values with the nonionic surfactant were ne $=24$ and ne $=53$ for GFP and R-PE, respectively. The corresponding theoretical nt for GFP and R-PE were 24 and 59, 
respectively. Theoretical and experimental peak capacities agree well. Peak capacity numbers were also comparable to free-flow and microchip IEF which are between 23 and 48 [91, 137]. However, cIEF results analyzed with precision MS or similar, more costly analytical tools report peak capacities 1 to 2 magnitudes larger [196, 197].

Numerical comparisons of $\mathrm{p} /$ difference and peak capacity indicate that SIEF, with additional optimizations, has the potential to approach the resolving power of other IEF platforms. The three to seven fold increase in resolution observed for GFP and R-PE with F-108 nonionic surfactant is promising. Further, the presence of surfactant increased the GFP peak capacity by $\sim 3$ fold and R-PE by 1.3 times. These results are consistent with the intensified focused bands and suppressed $\mathrm{pH}$ gradient compression and cathodic drift visually observed under the microscope. Thus, further investigation of additional surfactants and additives is warranted to further optimize sIEF.

\subsection{Conclusions}

This paper is the first report of surface isoelectric focusing conducted at a scale of 100 s of microns. This 10 to 100 -fold reduction in gel length enables a 10 -fold reduction in power requirements and a 100-fold improvement in sample consumption. The sIEF technique builds upon prior protein focusing knowledge in slab gel IEF and $\mu$ IEF to engineer a more powerful, versatile, surface-accessible IEF platform. This work demonstrates protein separation and focusing at a scale two orders of magnitude smaller than previous reports with roughly equivalent resolution.

The acrylamide gel lines can be readily printed on normal microscope glass slides using surface-printing techniques. Our results demonstrate that $\mathrm{pH}$ gradients can be successfully and reproducibly generated in $300 \mu \mathrm{m}$ long, $60 \mu \mathrm{m}$ wide, and $\sim 35$ $\mu \mathrm{m}$ tall and curved gels using broad range Pharmalyte ${ }^{\mathrm{TM}}(\mathrm{pH} 3-10)$. The $\mathrm{pH}$ gradient was verified via ratiometric $\mathrm{pH}$ sensitive and $\mathrm{pH}$ insensitive fluorescent dyes. Further, $0.9 \mathrm{ng}$ of GFP and R-PE protein samples $(0.5 \mathrm{mg} / \mathrm{mL})$ were 
separated and focused to $\mathrm{pH} \sim 4$ and $\mathrm{pH} \sim 6$ consistent with their $\mathrm{p} /$ values. $\mathrm{pH}$ gradient stabilization was demonstrated in 2 minutes while complete protein focusing was demonstrated within 10 minutes and successfully imaged in real time via fluorescent video microscopy. Electric field strengths and carrier ampholyte concentrations were investigated to determine optimized experimental conditions of $2 \% \mathrm{w} / \mathrm{v}$ PharmalyteTM and $200 \mathrm{~V} / \mathrm{cm}$. Focused band results elucidated challenges with $\mathrm{pH}$ compression and band distortions within the SIEF device. Thus, trials utilizing nonionic Synperonic $®$ F-108 surfactant within the gel revealed three to seven-fold improvements in protein separation efficiencies and in the sIEF resolving capability. Further investigations are warranted to fully explore ion accumulation effects and EOF to alleviate $\mathrm{pH}$ compression, band distortions, and protein precipitation; these will ultimately improve sIEF performance.

In summary, this new sIEF approach can transform the ease and versatility of nanoscale protein analysis by rapidly generating $\mathrm{pH}$ gradients and focus proteins on versatile and easily accessible glass surfaces. To achieve comparable focusing and resolving power, sIEF technique requires 10-100 times smaller applied voltages, and only $0.002 \mu \mathrm{L}$ of sample instead of the 10-20 $\mu \mathrm{L}$ in cIEF or gel IEF. The $\sim 100$-fold reduction in gel length presented in this work enables a 10 -fold reduction in power requirements and a 100-fold improvement in sample consumption. The supporting glass slides can be easily cleaned for reusability in excess of 50 runs. In addition, the surface printing will enable rapid construction of customizable sIEF gels that can be integrated into protein array libraries for specialized and/or orthogonal separations. The surface geometry also enables easy accessibility for spot picking tools in tandem with secondary protein analysis such as MS and MALDI-TOF. These advantages make sIEF attractive for future portable, user-friendly, in situ protein separations. 


\section{Surface Isoelectric Focusing (sIEF) Optimization and Application for Hemoglobin Variants Separation with Narrow Range pH Gradient ${ }^{3}$}

\subsection{Abstract}

Isoelectric focusing (IEF) plays an important role in amphoteric biological molecule pre-fractionation. For modern pre-fractioning techniques, sample species grouping, quick processing, and easy sample accessibility are required. Recently developed surface isoelectric focusing (sIEF) has characteristics that include quick analysis, a friendly sample accessing interface, and device multi-time usage. These attributes make sIEF a platform biomolecule pre-fractionation tool. This work expands sIEF capabilities for protein variant identification and fractionation to narrow $\mathrm{pH}$ ranges. Unpolymerized acrylamide gel lines were printed between parallel electrodes, then allowed to polymerize in situ. Narrow range carrier ampholyte Pharmalyte ${ }^{\mathrm{TM}}$ was used, along with a DC electric field, to generate a $\mathrm{pH}$ gradient ranging from 6.7 to 7.7 across the printed gel line. Fluorescent isoelectric point $(p /)$ markers at $6.8,7.2$ and 7.6 were used to characterize the established $\mathrm{pH}$ gradient. Results demonstrate rapid and stable narrow range $\mathrm{pH}$ gradients on the sIEF device with good reproducibility. Hemoglobin $(\mathrm{Hb})$ variants $A, S$ and $F$ were successfully separated with peak capacities around 50 . Focused bands displayed curvature, and were attributed to electrolysis byproducts distorting the gel gradient at the edges. To impede electrochemical reactions at the electrode surfaces, the entire sIEF device was coated with a $50 \mathrm{~nm}$ dielectric $\mathrm{HfO}_{2}$ thin film. The modified sIEF device separated $\mathrm{Hb}$ variants with $50 \%$ higher band peak capacities. Thus, $\mathrm{HfO}_{2}$ modification proved to be a promising technique for sIEF protein pre-fractionation. Surface access attributes will likely enable more

3 The material contained in this chapter is in preparation for submission to Biomicrofluidics 
seamless SIEF integration with secondary-analysis tools such as orthogonal electrophoretic mobilization to MS, MALDI-TOF, etc.

\subsection{Introduction}

Rapid, highly efficient screening of complex biomolecule mixtures, such as hemoglobin screening to identify variant/abnormal protein forms, which involves the discernments of structural variation from identical heme groups in molecular level. Also difficulties including identifying highly unstable hemoglobin that may manifest clinically as hemolytic anemia or thalassemia, or small amounts of variant hemoglobin that might not be detected by diagnostic techniques used in most clinical laboratories, require multistep separation/identification runs with the integration of different technologies. For the first-step screening tool, which plays as the coarse filter in the very beginning, accurate identification and quantification of fractionates are crucially important. The majority of pharmaceutical and biomedical analysis protocols utilize chromatography, gel or capillary electrophoresis for the first step biomolecule fractionation followed by mass spectrometry as the fine analysis tool [132, 198-200]. However, as prefractionation tools, conventional electrophoresis and chromatography will encounter limitations including long processing times, large sample volumes, and labor intensive protocols $[68,88]$. Furthermore, the conventional pre-fractionation tools do not translate readily to time sensitive and space confined situations such as emergency or battlefield analysis. Thus, modifications and improvements to conventional fractionation and separation techniques for biosample pre-screening are urgently in need.

Microfluidic chip-based IEF ( $\mu$ IEF) has been investigated and developed for more than a decade. With microfabrication techniques becoming more established, the focus of $\mu \mathrm{IEF}$ has transitioned from device design to real test applications [88]. Many biological/biomedical applications have found $\mu \mathrm{IEF}$ attractive as a first dimension fractionation tool leveraging amphoteric biomolecules focusing to unique $\mathrm{pl}$. This feature allows different amphoteric samples to be grouped and concentrated from complex analyte mixtures. Sample loading can also be reduced 
from the typical 10 20 $\mu \mathrm{L}$ required from conventional electrophoresis to $\sim 0.1 \mu \mathrm{L}$ in $\mu \mathrm{IEF}$ as a result of smaller operating scales [57, 133]. Lastly, miniaturized devices reduce the $\mu \mathrm{IEF}$ operation times [88]. Additionally, $\mu \mathrm{IEF}$ 's decreased device size does not compromise protein separation resolutions; therefore, commercial carrier ampholytes in $\mu \mathrm{IEF}$ efficiently resolves proteins with $\mathrm{p} /$ differences as small as $10^{-}$ ${ }^{1}-10^{-2}[68,87,88,91]$.

Other first dimension separation tools, such as chromatography-based capillary IEF, remain competitive due to high resolution and reproducibility. As a mechanism, ampholytic samples are either mixed together with carrier ampholytes or injected into the capillary to cause simultaneous $\mathrm{pH}$ gradient generation and IEF separation, or injected into a carrier ampholytes pre-filled capillary to run the separation. The sample injecting, voltage, and pressure controls are usually automatic, and all the capillaries and carrier ampholytes are commercial products; these advantages make the tools robust, and they are still being employed as widely accepted benchmark technologies [64, 201]. However, those tools require external forces to mobilize samples down the enclosed capillaries to detectors which increase dispersion [68, 202]. Also the cost, complicated operation, and large sample volume requirements are constraints of chromatography-based tools. While in another technique micro IEF ( $\mu \mathrm{IEF})$, samples are directly loaded into separation channels via diffusion or capillary forces, namely, no elaborate sampling technique required to keep analysis results consistent [132].

Despite $\mu \mathrm{EFF}$ advantages of fast and simple biomolecule fractionation without compromising resolution, the tool has issues that impede further development including $\mathrm{pH}$ gradient instability, reaction byproducts, mass transport, and sample access post separation. With more than 600 chemical entities and at least one thousand isoforms, carrier amphoteric molecules are oriented by a DC electric field to form a continuous $\mathrm{pH}$ gradient [21]. These amphoteric molecules experience anodic/cathodic drift and gradient compression due to the prolonged focusing time and high applied DC voltage $[155,164]$. These unstable $\mathrm{pH}$ gradient phenomena reduce resolvability and resolution of the protein separations. Also, Faradaic 
reaction (sometimes observed as electrolysis) at electrode surfaces alter the ionic composition of the gel media. Mass transport via electroosmotic flow (EOF) or electric field mediated migration of ionic species other than the amphoteric proteins can also alter local conditions [46, 203, 204], thus altering the established $\mathrm{pH}$ gradient $[91,164]$. EOF can be attenuated, but remains inevitable in $\mu$ IEF [205207]. Despite $\mu \mathrm{IEF}$ 's power, the tool has remained a research novelty without mainstream adoption due in large part to difficulties involving automating sample injections and the cumbersome steps (EOF mobilization to a deliberate range to reduce focusing time without sample over dispersion; sample modifications to suppress sample/device interface reaction; multilayers/channels design to improve resolving power, etc. $[92,93,127,132,137]$.) Also $\mu \mathrm{IEF}$ needed to recover the focused samples from the short enclosed channels for secondary analysis. Therefore, the combination of $\mu$ IEF's advantages of scale with clEF's advantages of $\mathrm{pH}$ gradient stability and focused sample recovery would yield an extremely powerful high-resolution separation tool.

We recently demonstrated surface isoelectric focusing (SIEF) [208] whereby small scale separations were conducted on sample accessible surfaces. Surface isoelectric focusing was conducted in a $60 \mu \mathrm{m} \times 300 \mu \mathrm{m}$ microprinted polyacrylamide gel line spanning two thin-film electrodes on a glass slide. The present work demonstrates improved $\mathrm{pH}$ gradient stability and SIEF resolutions achieved within narrow range $\mathrm{pH}$ 6.7-7.7 Pharmalyte $^{\mathrm{TM}}$ gels to separate hemoglobin variants. First, fluorescent peptide $\mathrm{p} /$ markers were utilized to quantify the position and shape of established $\mathrm{CA}$ pH gradients. $\mathrm{Hb}$ variants $\mathrm{A}, \mathrm{S}$ and $\mathrm{F}$, which have $\mathrm{p} /$ differences between 0.2 and 0.4 , were explored and separation efficiencies quantified via peak capacity and resolution. The impact of $\mathrm{pH}$ gradient stability was further explored via control of Faradiac reaction byproducts and chemical additives. Faradaic reaction byproducts from electrode surfaces were prevented from entering the gels by passivating the entire glass/microfabricated electrode surface with a $\mathrm{HfO}_{2}$ dielectric layer [181]. Chemical additives were incorporated into the gel to explore potential improvements to $\mathrm{pH}$ gradient drift and 
compression. For all conditions explored, quality of band resolvability was quantified and compared to identify optimal conditions for sIEF protein separations in nanoliter volume gels. This work demonstrates successful separations of proteins with hard to resolve $0.2 \mathrm{p} /$ differences in off-the-shelf narrow range Pharmalyte ${ }^{\mathrm{TM}}$ CAs.

\subsection{Materials and Methods}

\subsubsection{Chemicals:}

Fluorescent IEF markers (peptides with p/'s of 6.8, 7.2 and 7.6), acrylamide/bisacrylamide (29:1, 40\% w/v stock solution), and Synperonic ${ }^{\circledR} \mathrm{F}-108$ surfactant were ordered from Sigma-Aldrich (St. Louis, MO, USA). Tetramethylethylenediamine (TEMED), glycerin and ammonium persulfate (APS, $15 \% \mathrm{w} / \mathrm{v}$ solution polymerization catalyst of acrylamide/bis-acrylamide solution) were ordered from PlusOne (New York, NY, USA). Narrow range carrier ampholytes (Pharmalyte ${ }^{\mathrm{TM}}$ pH 6.7-7.7) were purchased from GE Healthcare (Pittsburgh, PA, USA). Simplicity Ultrapure 185 water system (E-pure water generator providing an $18.2 \Omega \cdot \mathrm{cm}$ resistivity product) and phosphate buffer saline (PBS) pre-mixed pellets were purchased from EMD Millipore (Billerica, MA, USA). Hemoglobin variants ( $\mathrm{Hb} A$, $\mathrm{S}$, and $\mathrm{F}$ mixture, diluted to $1 \mathrm{mg} / \mathrm{mL}$ with $1 \mathrm{mM}$ PBS solution) were purchased from Analytical Control Systems, Inc. (Fishers, IN, USA). Negative photoresist PR1-1000A and photoresist developer RD-6 were ordered from Futurrex (Franklin, NJ, USA). Silver epoxy is a commercial product of MG chemical (MG8331, 0.007 $\Omega \cdot \mathrm{cm}$ electrical resistivity and $0.90 \mathrm{~W} \mathrm{~m}^{-1} \cdot \mathrm{K}^{-1}$ thermal conductivity).

\subsubsection{Device Fabrication}

A $3 \times 7 \mathrm{~cm}^{2}$ glass chip with micro-patterned gold electrodes (100 $\mu \mathrm{m}$-wide spaced, $300 \mu \mathrm{m}$ apart) was prepared using soft photolithography followed by electro-vapor deposition (E-beam), details are as described in Chapter 3.2.2. In short, a glass slide was pre-cleaned and then spin-coated with PR-1000A photoresist. Electrode pattern transfer onto the glass slide was achieved by UV exposure under a computer designed, hollow caved mask, followed by RD-6 developing. Patterned metal electrodes were deposited using E-beam, the obtained metal layers contain 
$5 \mathrm{~nm}$ titanium followed by $150 \mathrm{~nm}$ gold. Photoresist and excess metal were removed by acetone sonication.

$\mathrm{A} \mathrm{HfO}_{2}$ thin film was attached onto the metal patterned glass chip using sputter deposition (PE 2400 Sputter Tool-8 inch) by first pre-vacuuming the chamber to $3 \times 10^{-7}$ Torr, and then applying $700 \mathrm{~W}$ plasma with $18 \mathrm{sccm}$ Ar flow and $4 \mathrm{sccm} \mathrm{O} 2$ flow $\left(7.4 \times 10^{-3}\right.$ Torr) to obtain a $50 \mathrm{~nm} \mathrm{HfO} 2$ layer at a $7 \mathrm{~nm} / \mathrm{min}$ deposition rate. During deposition, a cover glass slide was tapped on top of the electrode contact pads to prevent passivation. The $\mathrm{HfO}_{2}$ coated glass chip was uniformly heated on top of a 6 " Si wafer on a hotplate for $30 \mathrm{~min}$ at $250^{\circ} \mathrm{C}$ (temperature increase of $50^{\circ} \mathrm{C} / \mathrm{min}$ from room temperature to $250^{\circ} \mathrm{C}$ before cooling naturally) to enhance the rigidity and remove pinholes. External copper wires were attached on the completed glass chip contact pads using silver epoxy.

The sIEF gel printing protocol is the same as described in Chapter 4.2.3. In brief, a $60-\mu \mathrm{m} \times 300-\mu \mathrm{m}$ line was printed via a software-programmed surface printing tip (SPT) vector motion monitored in real time under microscope. The glass slide with micro-patterned electrode was pretreated in UVO cleaner, and the SPT was preloaded with a mixture of acrylamide/bis solution, narrow range Pharmalyte ${ }^{\mathrm{TM}}$, and APS. The overall device fabrication flowchart is described in Figure 5.1. 


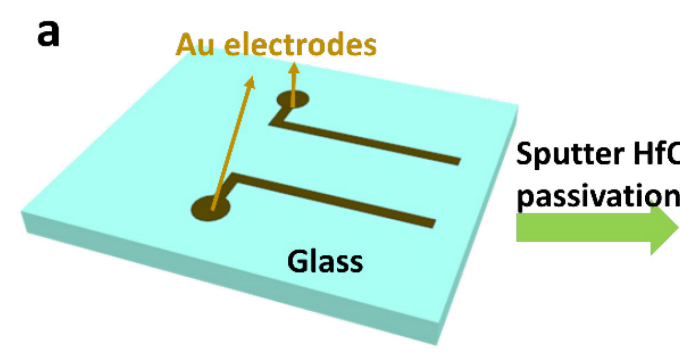

b
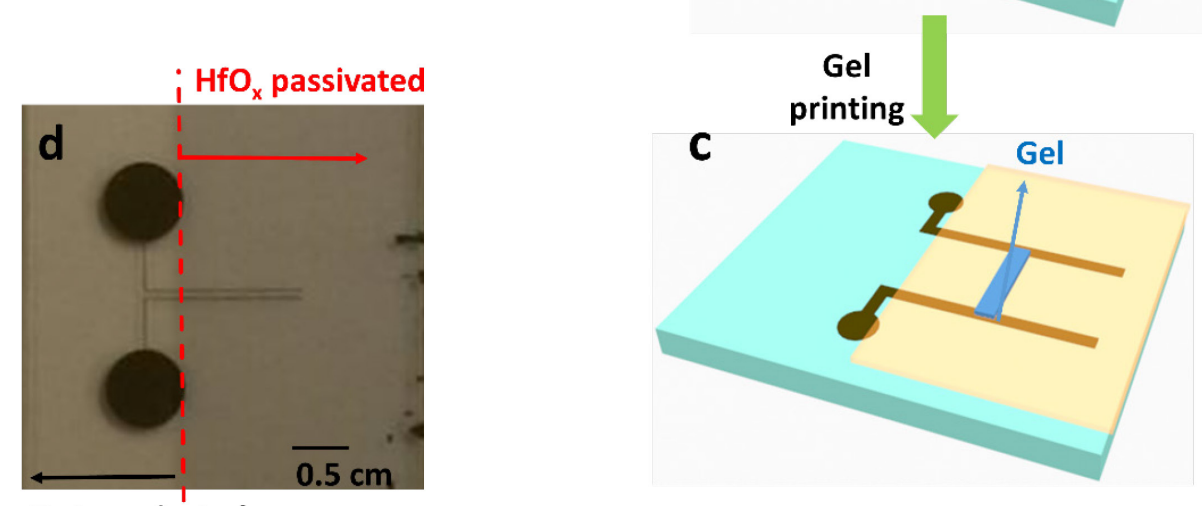

Not passivated

Figure 5.1 Configuration of the electrode-passivated surface-enabled IEF (sIEF) device. Device fabrication sequence includes a) $100 \mathrm{um}$ wide gold electrode pairs patterned onto a glass slide with circles representing contact pads, b) $\mathrm{HfO}_{2}$ passivation layer sputtered over the electrodes, c) Finished device with Pharmalyte ${ }^{\mathrm{TM}}$ co-printed with polyacrylamide gel spanning between gold electrode pairs spaced $300 \mu \mathrm{m}$ apart, and d) Image of the completed sIEF device. Gels were run with and without the $\mathrm{HfO}_{2}$ layer. Gelation occurred in-situ; a $\mathrm{pH}$ gradient formed within the gel upon electric field application.

\subsubsection{Experiment Set-up}

Fluorescent IEF markers ( $\mathrm{p} / \mathrm{s}$ of $6.8,7.2$, and 7.6) were focused using sIEF for $\mathrm{pH}$ gradient calibration. Pre-mixed IEF markers $(1: 1: 1 \mathrm{v} / \mathrm{v}$ ratio, with final concentrations of $0.33 \mathrm{mg} / \mathrm{mL}$ for each species) were printed on top of the preprinted sIEF gel line by SPT and then allowed 5 minutes for uniform sample diffusion throughout the gel. A $200 \mathrm{~V} / \mathrm{cm}$ electric field was applied through the gel while images of the IEF markers movement were recorded at 30 second intervals 
over 15 minutes to monitor activity. Intensity profiles at the gel middle zone (analysis area of $10 \times 300 \mu \mathrm{m}^{2}$ ) were obtained using Image $\mathrm{J}$.

The IEF experimental conditions for $\mathrm{Hb}$ variants were similar to that of IEF markers. $\mathrm{Hb}$ variants $\mathrm{A}, \mathrm{S}$ and $\mathrm{F}$ were premixed to final concentrations of $0.6 \mathrm{mg} / \mathrm{mL}, 0.2$ $\mathrm{mg} / \mathrm{mL}$ and $0.2 \mathrm{mg} / \mathrm{mL}$, respectively. The $\mathrm{Hb}$ mixtures were loaded onto preprinted gel with SPT. The $\mathrm{Hb}$ imaging was processed under bright field illumination to obtain well-visualized images and image intensity profiles. The raw microscope images were rendered first in negative colors and analyzed by Image $\mathrm{J}$ before being processed with contrast and brightness enhancement to visualize the focused bands as shown in Figure 5.2.
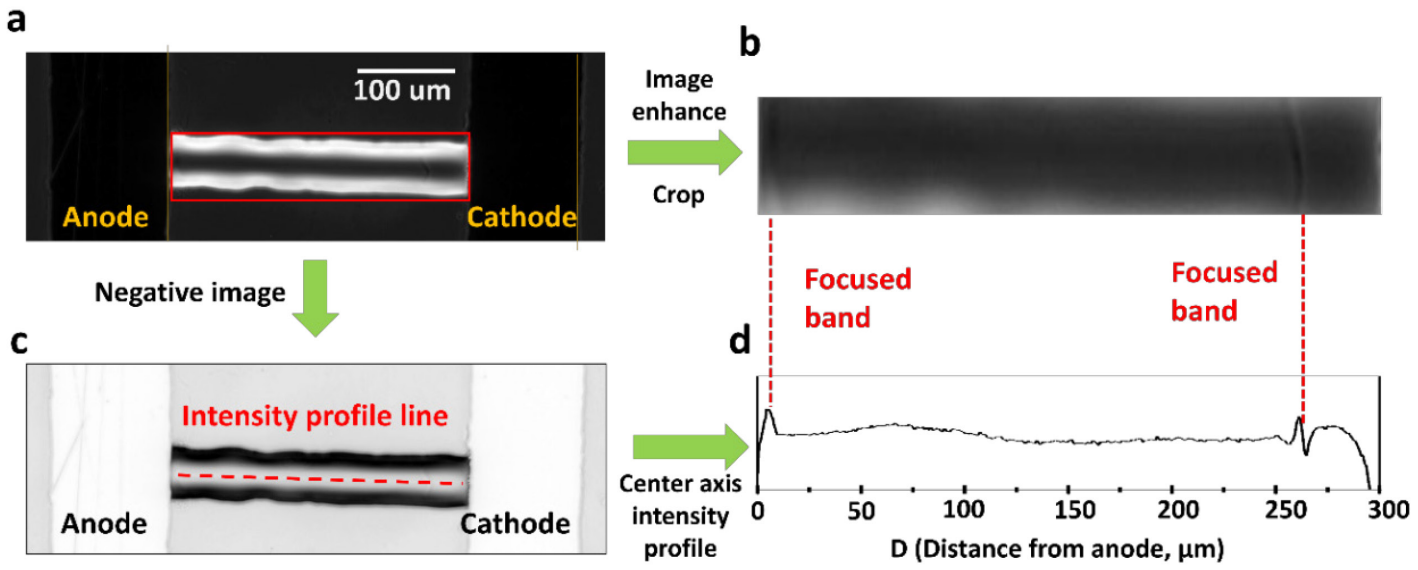

Figure 5.2 Demonstration of image processing and analysis procedures. a) The gel area spanning between the electrodes in the raw 20X microscope image was processed via brightness and contrast enhancement (b) to accentuate the focused bands. c) The image was also inverted and the negative image utilized for (d) intensity profile analysis. a) Raw microscope image, b) Enhanced image in gel area, c) Negative image for intensity analysis and d) Corresponding intensity profile. Focused proteins are shown as peaks in the profile and aligned with the enhanced image bands. 


\subsection{Results and Discussions}

\subsection{1 pH Gradient Establishment and Calibration}

In previous work, we reported the establishment and calibration of broad range $\mathrm{pH}$ gradients in a similarly constructed sIEF device. The $\mathrm{pH}$ distribution along the printed gel line was correlated to fluorescent intensity of $\mathrm{pH}$ sensitive dyes FITC and TRITC. However, FITC's working pH range ( $\mathrm{pH}$ 3.6-8.9) was too broad to be an effective indicator for the narrow $\mathrm{pH}$ range $(\mathrm{pH}$ 6.7-7.7) investigated herein [209]. Thus, we utilized an alternative $\mathrm{pH}$ calibration method adapted from conventional IEF - namely to focus samples with known isoelectric point to infer $\mathrm{pH}$ locations and thus the gradient $[64,204,210]$. Premixed fluorescent IEF markers with p/ values of 6.8, 7.2 and 7.6 were loaded onto sIEF gels and focused at $200 \mathrm{~V} / \mathrm{cm}$. The focused band positions were imaged and the position recorded as described in Figure 5.2, then correlated to $\mathrm{pH}$ as shown in Figure 5.3. Figure 5.3a demonstrates an enhanced contrast microscope image of IEF markers after 10 minutes of focusing in addition to the corresponding intensity profile. The fluorescent image illustrates three IEF markers focused at different locations; furthermore, the intensity profile more easily illustrates the focused bands reflected as peaks. In Figure 5.3b, five individual repeats were compiled and a correlation between $\mathrm{pH}$ and location was fitted using a non-linear Harrison model (Equation 5.1) where $D$ is the distance from the anode. This fitting reveals that the established

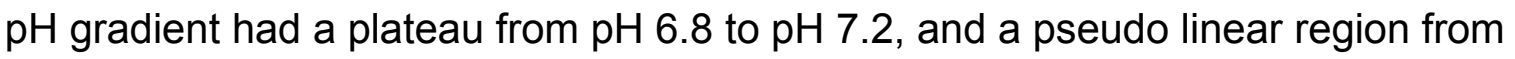
$\mathrm{pH} 7.2$ to $\mathrm{pH}$ 7.6. This pseudo linear region offers optimal separation conditions for proteins with $\mathrm{p} /$ values greater than 7 . However, separations will not be ideal for proteins with $\mathrm{p} /<7$.

$$
\mathrm{pH}=\frac{1}{\mathrm{a}+\mathrm{bD}}
$$

The narrow range $\mathrm{pH}$ 6.7-7.7 gradient followed a power law dependence in equation 1 while the broad range $\mathrm{pH}$ 3-10 gradient previously described followed a slightly different power law distribution $\mathrm{pH}=\left(2.57-\left(9.58 \times 10^{-6}\right) \mathrm{D}^{2.17}\right)^{-1}[180]$. Deviations from linear $\mathrm{pH}$ gradients reduces the ease, identification, and 
separation efficiencies of proteins. Therefore, sIEF modifications via electrode surface passivation and chemical additives were systematically explored to alter the $\mathrm{pH}$ gradient into a flatter $\mathrm{pH}$ distribution over position. The $\mathrm{pH}$ fit variables under conditions of blank control, passivation only, surfactant only and passivation + surfactant are summarized in Table 5 and discussed separately below.

Table 5 The $\mathrm{pH}$ fit, Equation 5.1, variables under different sIEF device modifications

\begin{tabular}{|c|c|c|c|}
\hline \multicolumn{4}{|c|}{ Equation 5.1 $1^{*}: \mathrm{pH}=\frac{1}{\mathrm{a}+\mathrm{b} D^{\mathrm{c}}}$} \\
\hline $\begin{array}{l}\text { Device modification type, Narrow Range } \\
\text { Pharmalyte }\end{array}$ & a & b & c \\
\hline No passivation, no additive (blank control) & 0.15 & $-9.55 \mathrm{E}^{-18}$ & 6.17 \\
\hline $\mathrm{HfO}_{2}$ passivation, no additive & 0.15 & $-3.52 \mathrm{E}^{-14}$ & 4.77 \\
\hline No passivation, F-108 & 0.15 & $-1.01 \mathrm{E}^{-16}$ & 5.78 \\
\hline $\mathrm{HfO}_{2}$ passivation, $\mathrm{F}-108$ & 0.15 & $-3.87 \mathrm{E}^{-15}$ & 5.14 \\
\hline $\begin{array}{l}\text { Broad Range Pharmalyte } \\
\text { No passivation, no additive }\end{array}$ & 2.57 & $-9.58 E^{-6}$ & 2.17 \\
\hline
\end{tabular}

${ }^{*} \mathrm{D}$ : distance from anode, $\mu \mathrm{m}$ 

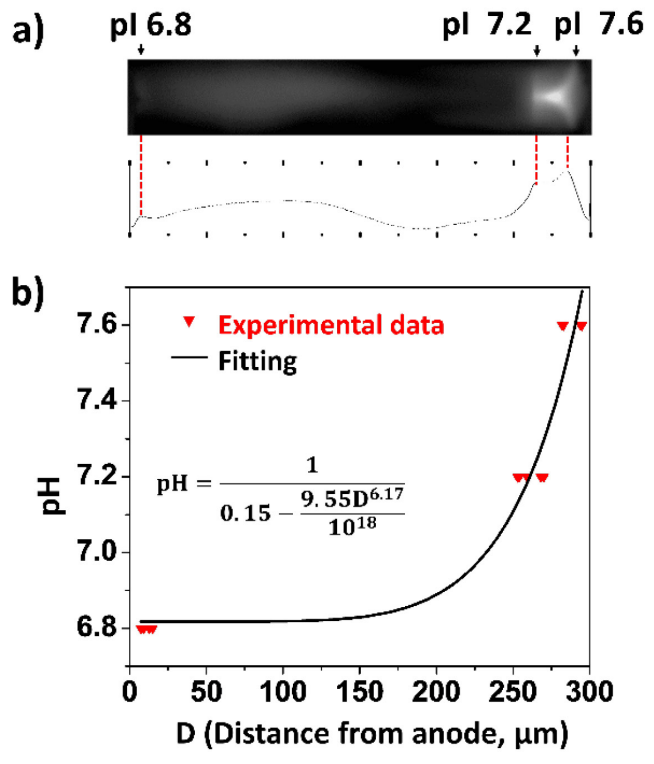

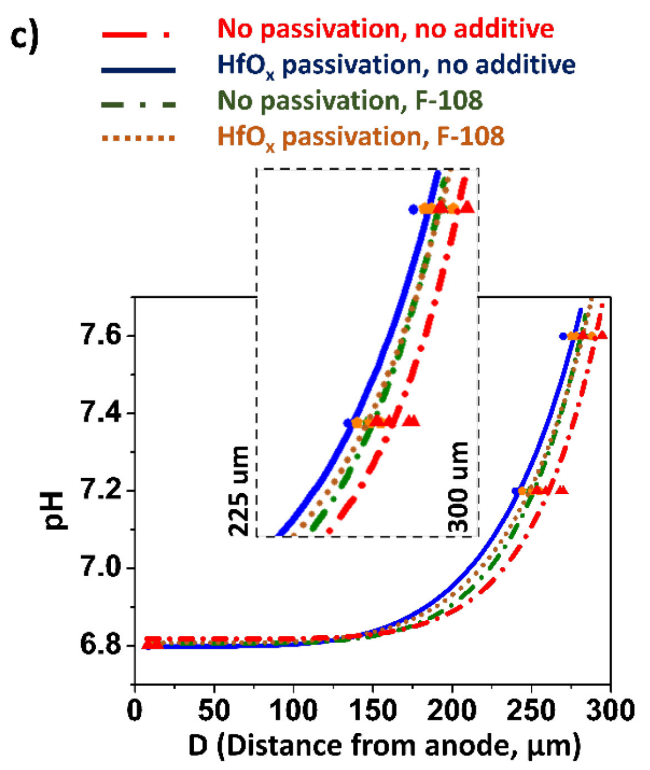

Figure 5.3 Demonstration of image processing and analysis procedures. a) The gel area spanning between the electrodes in the raw 20X microscope image was processed via brightness and contrast enhancement (b) to accentuate the focused bands. c) The image was also inverted and the negative image utilized for (d) intensity profile analysis. a) Raw microscope image, b) Enhanced image in gel area, c) Negative image for intensity analysis and d) Corresponding intensity profile. Focused proteins are shown as peaks in the profile and aligned with the enhanced image bands.

\subsubsection{SIEF Modifications to Improve the PH Gradient: Electrode}

\section{Passivation}

In microscale electrokinetics, including IEF, electrolysis of water at electrode surfaces produces $\mathrm{H}^{+}$and $\mathrm{OH}^{-}$that can cause non-ideal phenomena including $\mathrm{pH}$ compression and $\mathrm{pH}$ gradient instabilities [4, 91, 92, 155, 164]. The ionic electrolysis products move electrophoretically and thus accumulate within the IEF gels near the oppositely charged electrodes. These ion accumulations lead to increasingly acidic or basic local environments near the anode and cathode, respectively. Local acidic/basic conditions interfere with carrier ampholyte stacking 
causing gradient compression, drift or secondary electrohydrodynamic forces [91, $155,164]$.

In any lab-on-a-chip device with direct contact between electrodes and aqueous media, electrolysis at the electrode surfaces contributes protons and hydroxides into the solution [126]. Equations 5.2 and 5.3 show Faradaic electrolysis reactions at the anode and cathode, where $E_{\text {eq }}$ is the standard equilibrium electrode potential:

Anode: $\mathrm{H}_{2} \mathrm{O} \rightarrow 1 / 2 \mathrm{O}_{2}+2 \mathrm{H}^{+}+2 e^{-}$(in acid, $\left.E_{e q}=1.23 \vee[211]\right)$

Cathode: $2 \mathrm{H}_{2} \mathrm{O}+2 e^{-} \rightarrow \mathrm{H}_{2}+2 \mathrm{OH}^{-}$(in base, $E_{\text {eq }}=-0.83 \mathrm{~V}$ [211])

IEF has traditionally utilized gold and platinum electrodes, for which the overpotential is less than 2V DC. However, the applied DC voltages to drive IEF separations are $6 \mathrm{~V}$ in our sIEF case while up to $1900 \mathrm{~V}$ for cIEF [63]. This means electrolysis reactions are inevitable at IEF electrodes. This work therefore explores a method to reduce/alleviate electrolysis for sIEF.

Electrode passivation (EP) is a convenient, but less explored method to reduce electrolysis. EP materials used within lab-on-a-chip devices include polymer and dielectric coatings. The most common polymers are PDMS and photoresist [212214] whose shortcoming include durability and coating uniformity. Examples of dielectric coatings include silicon, silicon dioxide, and $\mathrm{HfO}_{2}$ [215-217]. The shortcomings of Si materials including opaqueness and low dielectric constant led to an alternative material, $\mathrm{HfO}_{2}$, being widely used in metal-oxide-semiconductor and ion sensitive field effect transistors (MOSFET and ISFET, respectively) and in electrochemical research, but has been used in a limited capacity in microfluidic devices. $\mathrm{HfO}_{2}$ features outstanding chemical stability, high dielectric constant as well as favorable optical characteristics [218-220]. To the best of our knowledge, only six published lab-on-a-chip devices have employed $\mathrm{HfO}_{2}$ for electrode modification [187, 216, 221-225]. For example, $\mathrm{HfO}_{2}$ passivation layers on aluminum electrodes of a complementary metal oxide semiconductor (CMOS) biosensor demonstrated current leakages less than $40 \mathrm{nA} / \mathrm{cm}^{2}$ with applied 
voltages up to $6 \mathrm{~V}$ [216]. Further $\mathrm{HfO}_{2}$ coating of glass was demonstrated in a square $500 \mu \mathrm{m}$ capillary subsequently used for waveguides and micro-optics [226]. Our group previously demonstrated a $150 \mathrm{~nm} \mathrm{HfO} 2$ film deposited over gold electrodes on glass slides, which prevented Faradiac reactions at the electrode surface [181]. $\mathrm{HfO}_{2}$ has proven to be relatively easy to deposit, and has demonstrated stability under a wide range of voltages; therefore, it was selected as a viable material to explore reduced ion production into sIEF gels.

To explore $\mathrm{HfO}_{2}$ electrode passivation, $\mathrm{pH}$ gradients of uncoated and coated devices were compared. A pH gradient was obtained for the $\mathrm{HfO}_{2}$ coated SIEF device (section 3 ) as shown in Figure 5.3c via the blue solid line. Equation 1 was utilized to fit power law parameters to the $\mathrm{p} /$ markers location; these parameters are summarized in Table 5. The gradient between $\mathrm{pH} 6.8$ to $\mathrm{pH} 7.6$ extended from $150 \mu \mathrm{m}$ to the cathode at $300 \mu \mathrm{m}$ when $\mathrm{HfO}_{2}$ was present. Without $\mathrm{HfO}_{2}$ passivation, the same gradient spanned $200 \mu \mathrm{m}$ to $300 \mu \mathrm{m}$. Considering the typical focused band width is ca. $5 \mu \mathrm{m}$, the $50 \%$ increase $(50 \mu \mathrm{m})$ in functional separation space in the gel means up to 7 additional protein bands can be resolved assuming a $2 \mu \mathrm{m}$ gap between each band. The mechanism behind the extended $\mathrm{pH}$ gradient via $\mathrm{HfO}_{2}$ passivation is reduction of electrolysis reactions at the electrodes whereby free electrons are unable to access the metal for redox reactions to split water into hydrogen and oxygen gases as well as $\mathrm{H}^{+}$and $\mathrm{OH}^{-}$ions (Equations 2 and 3). As a consequence, fewer free ions are released into the gel thus reducing cation/anion accumulation and gradient compression. This mechanism plays a substantial role in $\mathrm{pH}$ gradient establishment and stability [91, 155, 164]. The electric field in the gel is attenuated by $\sim 44 \%$ with the dielectric coating [227]which increases the time required to fully establish the $\mathrm{pH}$ gradient from $7 \pm 1$ min to 10 $\pm 1 \mathrm{~min}$. Thus, the beneficial $\mathrm{pH}$ gradient spatial elongation observed with minor increases in time to establish the $\mathrm{pH}$ gradient is attributed to limiting ion production from the electrodes. 


\subsubsection{SIEF Modifications: Chemical Additives}

Separation efficiency can be increased by reducing $\mathrm{pH}$ gradient compression and improving focused band shape. Prior work has demonstrated that band distortions in IEF can be reduced via chemical additives, as demonstrated in CIEF as well as in our recently demonstrated broad range sIEF [207, 228]. Irregular band shape and/or band distortion are due to factors including EOF, protein precipitation, protein-surface interactions, and protein-gel interactions. The first three factors are concerns in both gel IEF and free-flow IEF while the latter is only a concern in gel IEF. To minimize EOF, surface modifications are common in cIEF with channel coatings or chemical additive techniques translated into $\mu \operatorname{IEF}[4,133,229-232]$. In PDMS channel free-flow $\mu \mathrm{IEF}$, EOF suppressants include methylcellulose (MC), polyvinylalcohol (PVA) and polyvinylpyrrolidone (PVP) [133, 205, 206], MC is the most common because it also helps alleviate PDMS/analyte interactions. However, EOF suppressants for quartz surfaces include non-ionic surfactant $F-108$, poly(L-

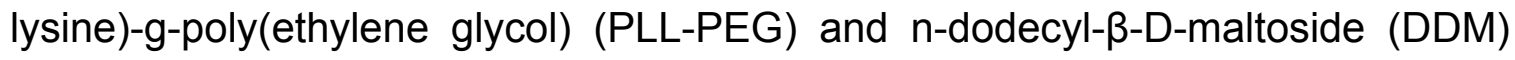
with $\mathrm{F}-108$ suppressing EOF mobility most effectively [228]. To enhance gel based IEF, surfactants have been used to simultaneously tune surface hydrophobicity/hydrophilicity and alter protein-gel interactions. Li's work demonstrated surface hydrophilicity changes by introducing cyclic olefin copolymer $(\mathrm{COC})$ to polyacrylamide gel, which reduced surface resistance and translated into higher IEF peak capacities [150]. Park et.al. demonstrated that nonionic surfactant F-108 facilitated larger acrylamide gel pores thus easing protein squeezing through pores [147]. Considering that non-ionic F-108 demonstrated benefits with quartz surfaces and polyacrylamide gels, our group thus co-printed F-108 with gels on glass for sIEF. Protein-surface interactions and precipitations were reduced and the band shape was improved[208].

Therefore, the $\mathrm{pH}$ gradient was obtained with and without F-108 as shown in Figure 5.3c; this comparison was completed both with and without $\mathrm{HfO}_{2}$ passivation. Table 5 provides parameters for Equation 14 to fit the data. Without $\mathrm{HfO}_{2}$ passivation, comparisons show that the $\mathrm{pH}$ distribution between $\mathrm{pH} 6.8$ and 
7.2 was spread over $125 \mu \mathrm{m}$ whereas the no surfactant control was $100 \mu \mathrm{m}$, which translates into a $25 \%$ increase in functional separation space. Surfactant-based $\mathrm{pH}$ gradient improvement $(25 \%)$ was not as effective as electrode passivation $(50 \%)$, and the $7 \pm 1$ min focusing time was nearly the same as the no surfactant, no passivation control.

The presence and absence of $\mathrm{F}-108$ was also conducted with $\mathrm{HfO}_{2}$ passivation under identical conditions as all prior experiments $(2 \% \mathrm{w} / \mathrm{v}$ narrow range Pharmalyte $^{\mathrm{TM}}$ and $200 \mathrm{~V} / \mathrm{cm}$ ). Unexpectedly, increases in the $\mathrm{pH}$ gradient spread was not additive. The $\mathrm{HfO}_{2}$ passivation, F-108 (brown dot line) in Figure 5.3c shows that the functional separation space was increased by only $30 \%$ with $9 \pm 1$ min focusing time. This is slightly better than F-108 only and worse than $\mathrm{HfO}_{2}$ passivation only. This could be attributed to differing F-108 surface modifications on $\mathrm{HfO}_{2}$ compared with glass, as well as EOF mobility differences between glass and $\mathrm{HfO}_{2}$.

\subsubsection{Hemoglobin Separation with Narrow Range pH sIEF}

After reproducibly demonstrating narrow range $\mathrm{pH}$ gradient formation, qualitative and quantitative protein sIEF could be evaluated. Hemoglobin variant mixtures containing $\mathrm{Hb} \mathrm{A}$ ( $\mathrm{p} / 6.9$ ), $\mathrm{Hb} F$ (7.1), and $\mathrm{Hb} \mathrm{S}$ (7.3) [112] were selected to test resolvability. $\mathrm{Hb}$ sIEF was operated under identical electric field conditions of 200 $\mathrm{V} / \mathrm{cm}$ with Pharmalyte ${ }^{\mathrm{TM}}$ concentrations of $2 \% \mathrm{w} / \mathrm{v}$. Separations were performed with and without a passivation layer and with and without F-108 additive.

Initially, $\mathrm{Hb}$ band focusing was imaged without device passivation and without chemical additives. Figure $5.4 a$ shows the raw microscope image and corresponding intensity profile of $\mathrm{Hb}$ bands after 7 minutes of focusing. For three repeats, $\mathrm{Hb}$ variants $A, F$, and $S$ were all successfully separated and focused into bands with focusing time deviating by less than one minute. The $\mathrm{Hb}$ variants were not fluorescently tagged to avoid alteration of the protein p/ point; the trade-off was reduced image contrast which was countered by closely coupling images with their corresponding intensity profiles. By applying Equation (1), the experimental p/ 
values of $\mathrm{Hb}$ variants $\mathrm{A}, \mathrm{F}$ and $\mathrm{S}$ were found to be $6.80,6.95$ and 7.04 , respectively (with \pm 0.003 error). These differ from the reported literature values by $0.10,0.15$, and 0.26 , respectively [112]. $\mathrm{Hb} A$ was the only variant whose $\mathrm{p} /$ positioned it in the plateau $\mathrm{pH}$ region. This facilitated a larger positional separation from $\mathrm{Hb} \mathrm{F}$ and $\mathrm{Hb}$ S, which were positioned at $219 \mu \mathrm{m}$ and $232 \mu \mathrm{m}$. An easily recognizable $\mathrm{Hb}$ mixture separation was obtained for the control conditions without passivation and without additives.

a) No passivation, no additive

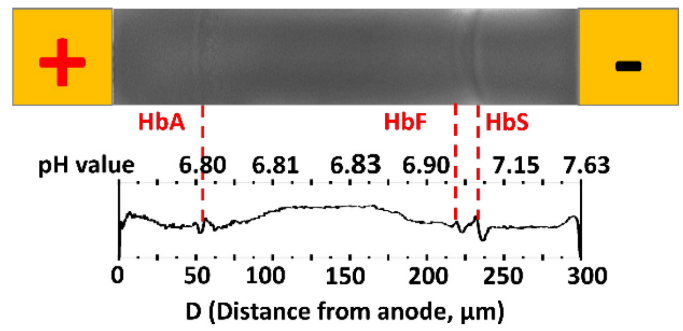

c) No passivation, F-108
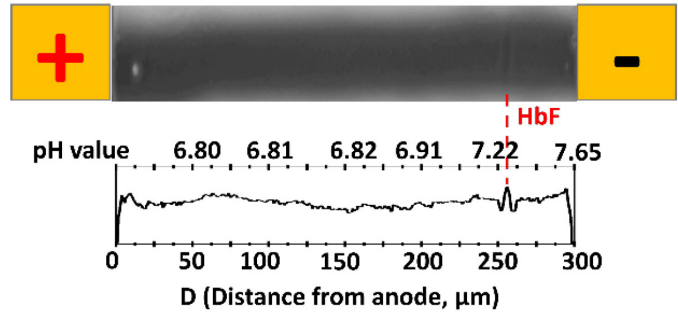

b) $\mathrm{HfO}_{\mathrm{x}}$ passivation, no additive

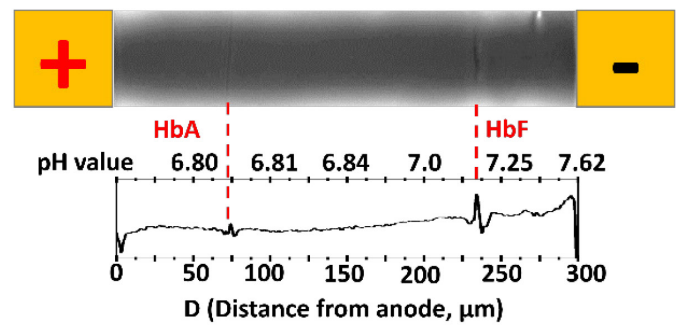

d) $\mathrm{HfO}_{x}$ passivation, F-108

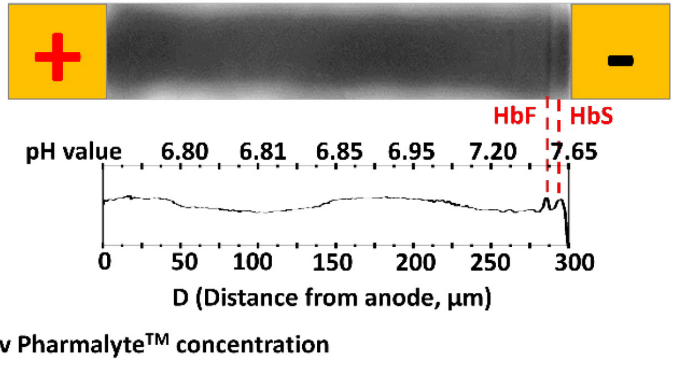

Figure 5.4 Microscope images and corresponding intensity profiles of sIEF-focused hemoglobin variants $A, S$ and $F$ with pl 6.97, 7.21 and 7.06, respectively. a) no electrode passivation, no additive, b) $50 \mathrm{~nm} \mathrm{HfO}_{2}$ passivated electrode, no additive, and c) no electrode passivation, $1 \%$ $\mathrm{w} / \mathrm{v}$ Synperonic ${ }^{\circledR} \mathrm{F}-108$. Although condition a) had defined bands, the surfactant and passivation layer improved band distortion with d) $50 \mathrm{~nm}$ $\mathrm{HfO}_{2}$ passivation, $1 \% \mathrm{w} / \mathrm{v}$ Synperonic ${ }^{\circledR} \mathrm{F}-108$. All experiments were conducted at $2 \% \mathrm{w} / \mathrm{v}$ narrow range Pharmalyte ${ }^{\mathrm{TM}}(\mathrm{pH}$ 6.7-7.7) and 200 $\mathrm{V} / \mathrm{cm}$ electric field.

Similar to $\mathrm{pH}$ gradient experiments, hemoglobin variants were investigated under different SIEF modification conditions. Figure $5.4 \mathrm{a}$ and $4 \mathrm{~b}$ compare focused images and intensity profiles without and with passivation and without additive; curved bands were obtained without $\mathrm{HfO}_{2}$ passivation while the sIEF device with 
passivation yielded straight bands. This result demonstrated a noticeable shape improvement with the presence of $\mathrm{HfO}_{2}$ coating, even though it took 2 additional minutes to focus the bands. Figure $5.4 \mathrm{c}$ illustrates band focusing with $1 \% \mathrm{w} / \mathrm{v} \mathrm{F}$ 108 without passivation; the F-108 also alleviated band distortion and the run time was similar to Figure 5.4a control ( $7 \pm 1$ min for 3 repeats). With both passivation and F-108 in Figure 5.4d, straight bands and minor increased focus time (9 91 $\mathrm{min}$ ) were observed, consistent with the separate dependency results. Similar to prior reports [147, 150, 228], F-108 improved band shape due to: 1) enlarged pores in acrylamide gel that ensured open routes for protein movement, 2) decreased protein-gel interactions, 3) reduced protein precipitation, and 4) reduced EOF at the glass/gel/liquid interface. Thus, the presence of both $\mathrm{HfO}_{2}$ and $\mathrm{F}-108$, either together or separate, improves interactions of the proteins with the gel and device surface resulting in improved band quality. 


\subsubsection{Pharmalyte ${ }^{T M}$ and Surfactant Concentration}

$\mathrm{HfO}_{2}$ passivation results demonstrated improved IEF band shape. Similar to prior work, Pharmalyte ${ }^{\mathrm{TM}}$ concentration has been shown to alter the shape of focused protein bands $[91,208]$. This effect was examined in narrow range sIEF with Pharmalyte ${ }^{\mathrm{TM}}$ concentrations of $2 \%, 3 \%$ and $4 \% \mathrm{w} / \mathrm{v}$. Results are shown in Figure 5.5 for $\mathrm{HfO}_{2}$ passivated devices. Raw microscope images illustrate that only $2 \%$ $\mathrm{w} / \mathrm{v}$ of Pharmalyte ${ }^{\mathrm{TM}}$ was capable of maintaining straight focused bands as shown in the insets. In $3 \%$ and $4 \% \mathrm{w} / \mathrm{v}$ Pharmalyte ${ }^{\mathrm{TM}}$, curved bands were again observed. Higher Pharmalyte ${ }^{\mathrm{TM}}$ concentrations introduce more amphoteric molecules in closer proximity causing an increase in ionic strength. This increases the current making $\mathrm{pH}$ gradient stabilization harder to achieve. This observation is consistent with broad range $\mathrm{pH}$ sIEF results [91].
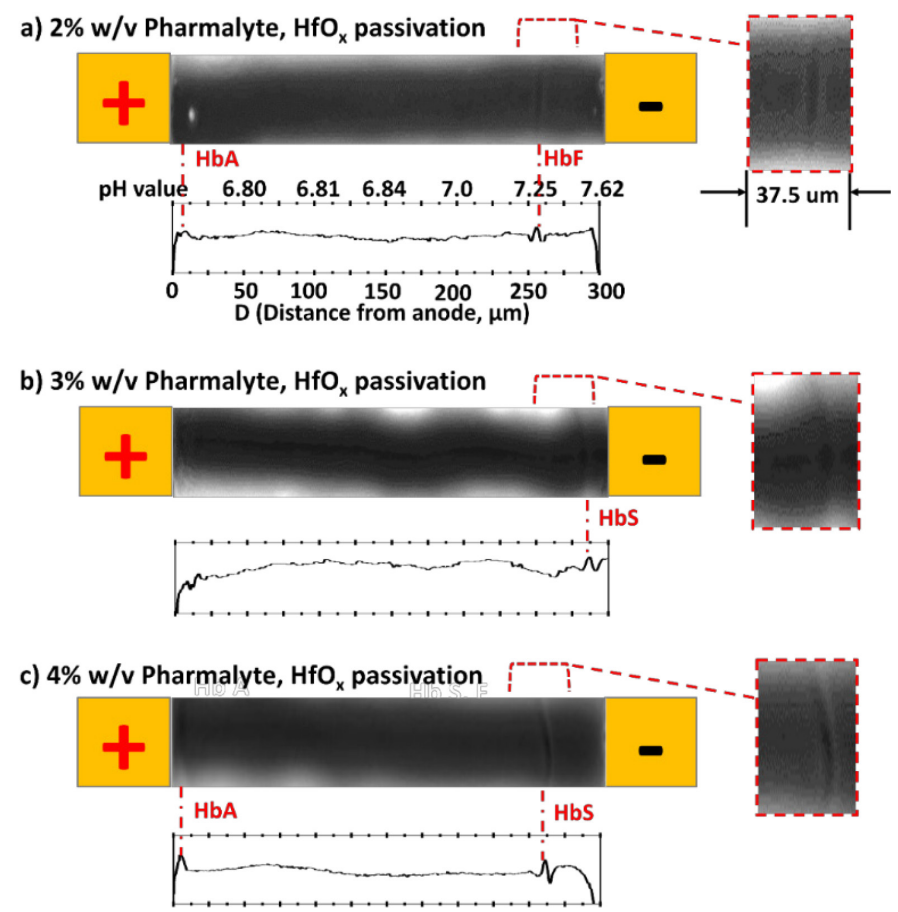

Figure 5.5 Pharmalyte ${ }^{\mathrm{TM}}$ concentration dependency on $\mathrm{HfO}_{2}$ coated device. $2 \%, 3 \%$ and $4 \% \mathrm{w} / \mathrm{v}$ Pharmalyte ${ }^{\mathrm{TM}}$ results are shown in a), b) and c), respectively. The most reproducible and straight bands were obtained with $2 \%$ Pharmalyte $^{\mathrm{TM}}$. As Pharmalyte ${ }^{\mathrm{TM}}$ concentration increased, distortion of focused band became more severe. 
Since the combination of $\mathrm{HfO}_{2}$ passivation and $\mathrm{F}-108$ achieved straight band focusing for two out of three $\mathrm{Hb}$ variants, an F-108 concentration dependency study was conducted to discern whether all $\mathrm{Hb}$ variants could be efficiently focused. Experiment conditions were kept identical except the concentration of F-108 was reduced to $0.5 \% \mathrm{w} / \mathrm{v}$. Figure 5.6 compares $\mathrm{F}-108$ concentrations whereby all three $\mathrm{Hb}$ variants resolved at $0.5 \% \mathrm{w} / \mathrm{v}$. $\mathrm{Hb} \mathrm{S}$ and $\mathrm{Hb} \mathrm{F}$ focused into sharp, straight bands while $\mathrm{Hb}$ A demonstrated a broader precipitated band. The peak capacities of $\mathrm{Hb}$ $\mathrm{F}$ and $\mathrm{S}$ were 51.7 and 77.5 , respectively. The broad $\mathrm{Hb} \mathrm{A}$ band enables identification, but peak capacity could not be reliably calculated. This improvement to identification of all three $\mathrm{Hb}$ variants is likely due to better surface-protein interactions in the presence of low concentrations of surfactants.

a) $0.5 \% \mathrm{~F}-108, \mathrm{HfO}_{\mathrm{x}}$ passivation

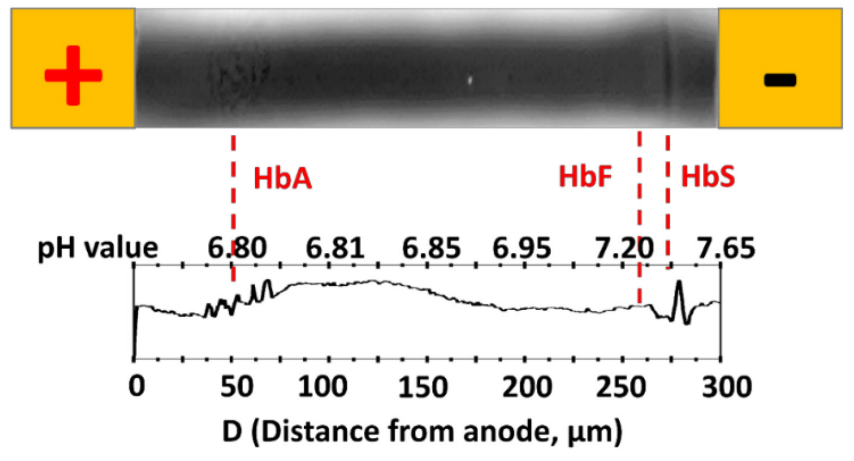

b) $1 \% \mathrm{wt} / \mathrm{v} \mathrm{F}-108, \mathrm{HfO}_{\mathrm{x}}$ passivation

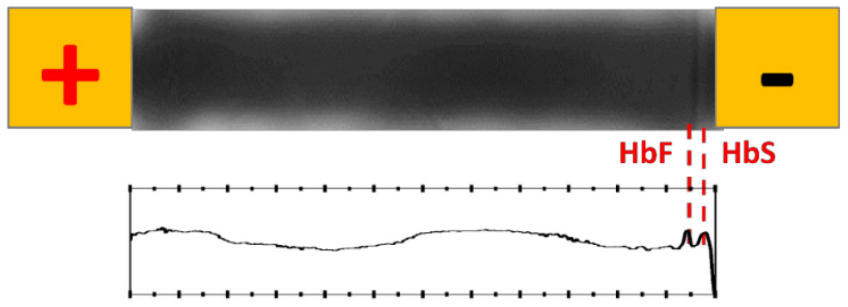

Figure 5.6 Surfactant concentration investigate on $\mathrm{HfO}_{2}$ coated device. F-108 concentration was controlled at a) $0.5 \% \mathrm{w} / \mathrm{v}$ and $\mathrm{b}) 1 \% \mathrm{w} / \mathrm{v}$. Bands were more optimally spaced with the $0.5 \% \mathrm{w} / \mathrm{v}$ surfactant concentration enabled resolving of $\mathrm{HbA}$ while $1 \% \mathrm{w} / \mathrm{v}$ did not. 


\subsubsection{Peak Capacity Comparison}

As described in Chapter 4.4.3, experimental peak capacity, $\mathrm{n}_{\mathrm{e}}$, is an important comparative criterion for IEF capability, which can be calculated from the intensity profile as shown in Equation 5.4. [106-108].

$$
\mathrm{n}_{\mathrm{e}}=1+\frac{\mathrm{L}}{\mathrm{w}}
$$

Where $w$ is the peak width taken at $4 \sigma$, and $L$ is fixed at $300 \mu \mathrm{m}$. While narrow peak widths are desirable, higher peak capacities mean more bands can be resolved. The experimental peak capacity calculation results for $\mathrm{Hb}$ variants $\mathrm{A}, \mathrm{F}$ and $S$ are 24.7, 60.5 and 27.0, respectively. Calculated theoretical peak capacities, $\mathrm{n}_{\mathrm{t}}$, as previously described [208], are $1.9,34.7$, and 44.3 , respectively. This low theoretical $\mathrm{Hb}$ A peak capacity is due to the flat $\mathrm{pH}$ gradient region from 0 to 150

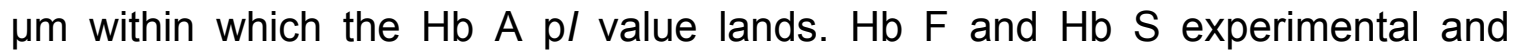
theoretical peak capacities are of similar magnitude with slightly greater experimental $\mathrm{Hb} F$ and slightly lower experimental $\mathrm{Hb}$ S peak capacities.

The experimental peak capacities for the $\mathrm{Hb}$ variants under each modification conditions are as shown in Table 6. As a result of superior band shape, the $\mathrm{HfO}_{2}$ passivated devices and F-108 added runs, yielded better peak capacity than devices without passivation or without F-108; however, the presence of $\mathrm{HfO}_{2}$ and $\mathrm{F}-108$ individually and together caused reduced discrimination of $\mathrm{Hb}$ variants. Only $\mathrm{Hb} \mathrm{A}$ and $\mathrm{Hb} \mathrm{F}$ bands were observed in passivation only experiments; only $\mathrm{Hb} F$ was focused with $\mathrm{F}-108$ only experiments. $\mathrm{Hb} \mathrm{F}$ and $\mathrm{Hb} \mathrm{S}$ focused when both $\mathrm{HfO}_{2}$ and F-108 were present, but significant compression near the cathode was observed. Thus, only the no passivation, no additive control was able to resolve all three $\mathrm{Hb}$ variants. 
Table 6 Peak capacity calculations of different sIEF passivation and additive conditions

\begin{tabular}{|c|c|c|c|}
\hline \multirow{2}{*}{ sIEF Modification } & \multicolumn{2}{|c|}{ Peak Capacity (experimental) } \\
\cline { 2 - 4 } & $\mathrm{Hb} \mathrm{A}$ & $\mathrm{Hb} \mathrm{F}$ & $\mathrm{Hb} \mathrm{S}$ \\
\hline Blank control & 24.7 & 60.5 & 27.0 \\
\hline $\mathrm{HfO}_{2}$ passivation only & 63.5 & 65.7 & - \\
\hline $1 \%$ w/v F-108 only & - & - & 69.8 \\
\hline $\mathrm{HfO}_{2}$ passivation, 1\% \\
w/v F-108
\end{tabular}

Peak capacity magnitude in SIEF was also compared with conventional IEF and proteomics analysis tools, as shown in Table 7. Chromatography-based tools such as MS and CIEF in tandem with secondary analysis still have better resolving capabilities with peak capacities ranging from $10^{2}$ to $10^{3}$. However, those tools remain higher in cost, laboratory tethered, and are more time consuming due in part to higher labor skill demands (sample preparation, pre and post IEF run setup). MS and CIEF are extravagant for most first dimension separations. Miniaturized, low-cost $\mu \mathrm{IEF}$ has 1 2 magnitudes smaller resolving power (peak capacities of $30 \sim 50$ ), but $\mu$ IEF is more adaptive for portable, fast analysis requirements and is highly suitable for most first dimension separations. SIEF has equivalent or even better resolving power than $\mu \mathrm{IEF}$, but boasts conveniences 
such as open sample access, smaller sample requirements, and simpler device fabrication. Therefore, sIEF has a potential niche utilization. Selective use of cIEF and MS as second dimension analysis tools following first dimension sIEF could, in many situations, provide the greatest resolving power with fewer resources.

Table 7 Peak capacity comparisons between mainstream techniques

\begin{tabular}{|c|c|}
\hline Technique & Peak Capacity \\
\hline MS [233] & $10^{3}$ \\
\hline $\begin{array}{c}\text { clEF followed by second dimension } \\
\text { analysis [122] }\end{array}$ & $10^{3}$ \\
\hline First dimension cIEF [234] & $10^{2}$ \\
\hline$\mu \mathrm{EEF}[91]$ & $30-50$ \\
\hline sIEF & Up to 70 \\
\hline
\end{tabular}

\subsection{Conclusions}

This work explored surface isoelectric focusing (sIEF) for narrow $\mathrm{pH}$ range Pharmalyte ${ }^{\mathrm{TM}}$ as an extension of our previous broad $\mathrm{pH}$ range sIEF work. The narrow range resolving power was later examined with a highly relevant medical diagnostic system of three hemoglobin variants, $\mathrm{Hb} \mathrm{A}, \mathrm{F}$, and $\mathrm{S}$ with hard to resolve $0.2 \mathrm{p} /$ differences. Attributes of sIEF demonstrated herein include straightforward gel printing via automatically controlled software, fast sample focusing and detection via video microscopy, low sample volume requirements due to gel size and nanoliter droplet printing, and surface accessibility for post sample treatment. By using commercial Pharmalyte ${ }^{\mathrm{TM}}$, a narrow range $\mathrm{pH}$ gradient from 6.7-7.7 was successfully established between two microfabricated electrodes spanned by a $300-\mu \mathrm{m}$-long polyacrylamide gel line. Fluorescent IEF markers with $\mathrm{p} /$ values of 6.8, 7.2 and 7.6 were utilized to correlate $\mathrm{pH}$ to gel position. These successfully generated and imaged $\mathrm{pH}$ gradients suggest that other narrowly confined $\mathrm{pH}$ 
ranges could be sustained in a sIEF gel by specifying the carrier ampholyte mixture for desired $\mathrm{pH}$ values.

Within the narrow range $\mathrm{pH}$ gradients, hemoglobin variants $\mathrm{Hb} \mathrm{A}, \mathrm{F}$ and $\mathrm{S}$ were successfully separated and identified. Discernments of the structural variations from heme groups was demonstrated with $12 \mu \mathrm{m}$ spatial separation of $\mathrm{Hb}$ bands in less than 10 minutes with sample volumes as low as $90 \mathrm{~nL}$. With the aim to improve sIEF resolving power, a dielectric $\mathrm{HfO}_{2}$ thin film was deposited to prevent direct contact between the electrode surfaces and the aqueous gel media. This reduced electrochemical reactions at the electrode surfaces to yield a more broadly distributed $\mathrm{pH}$ gradient with improved protein band shapes. This suggests passivation layers may be extendable to any IEF electrode to suppress electrolysis. Additionally, non-ionic surfactant F-108 was co-printed with the gel to improve protein/gel/surface interactions and successfully demonstrated improvements to the focused band shapes. Results from more than 3 repeats revealed that the most effective $\mathrm{Hb}$ variant separations - as judged via total focused bands and peak capacities - were achieved with a combination of $\mathrm{HfO}_{2}$ surface passivation, $0.5 \%$ $\mathrm{w} / \mathrm{v} \mathrm{F}-108$, and $2 \% \mathrm{w} / \mathrm{v}$ Pharmalyte ${ }^{\mathrm{TM}}$. Peak capacities improved from ca. 25 to ca. 70. sIEF peak capacities were comparable to prior $\mu \mathrm{IEF}$ results (peak capacities of 30 50) and one to two orders of magnitude smaller than clEF. This suggests that cIEF remains an ideal tool for the highest resolution applications where analysis time and expense is not a factor.

Furthermore, sIEF performance places it alongside $\mu$ IEF for time sensitive, space confined, portable, screening demands such as rapidly discerning structural variations of proteins for medical diagnostics and pharmaceutical synthesis. Additional attributes of sIEF beyond $\mu$ IEF include easier surface access, simpler device fabrication and device reusability up to $\sim 50$ times. In summary, miniaturized sIEF has sufficient resolving power and versatility to be adapted as a prefractionation tool for portable, fast analysis requirements that are highly suitable for most first dimension separations. Lastly, sIEF can be integrated with post- 
analysis tools for orthogonal separations and/or other more advanced biological molecule analysis. 


\section{Surface Isoelectric Focusing (sIEF) as Auxiliary Tool for Rapid Glycoprotein Pre-analysis ${ }^{4}$}

\subsection{Abstract}

As one type of the major components of mammalian cells, glycoprotein plays important role in fundamental pharmaceutical research. By performing glycobiology analysis, plenty of valuable information can be obtained including improve molecular stability, regulate physicochemical and pharmacological properties, and improve pharmacokinetics with better absorption and longer circulation times. Since molecular modifications in different glycoprotein domains can lead to different biological consequences, monitoring of those modifications are critical. Conventional proteomics tool such as HPLC and MS can offer a systematically examination and still be employed for glycoprotein modification checking. However, main drawbacks including complicated pre-sampling and preanalysis process and are always be the obstacle of rapid glycoprotein characterization. Isoelectric focusing (IEF) is an effective and widely used tool for amphoteric molecule pre-fractionation, which featuring fast sample species grouping by isoelectric points ( $p /$ values) and quick processing. The newly developed miniaturized surface isoelectric focusing (sIEF) in our group offers quick analysis as well as friendly sample accessing interface, which make it suitable for glycoprotein pre-analysis. This paper examines sIEF's capabilities to discern glycoprotein structural modification. Narrow range ampholytic $\mathrm{pH}$ gradients ranging from 6.7-7.7 were established within micro printed acrylamide gels using $200 \mathrm{~V} / \mathrm{cm}$ DC electric fields.. Modification of a monoclonal antibody mouse IgG glycoprotein was tested via sIEF before and after urea-induced denaturation and partial dithiothreitol (DTT) reduction glycoprotein major structure. The extent of molecular unfolding of mouse IgG was controlled by varying urea concentrations between 0 and $8 \mathrm{M}$. DTT concentrations were fixed at a previously optimized 25

${ }^{4}$ The material contained in this chapter is in preparation for submission to Electrophoresis 
$\mathrm{mM}$ to achieve protein reduction. Results revealed that molecular unfolding affects the subsequent IgG reduction levels, which can be successfully monitored via sIEF because unfolding and reduction of disulfide bonds alters the glycol protein's surface charge triggering a $\mathrm{p} /$ shift. With increased urea concentration, light chain $(\mathrm{LC})$ and heavy chain $(\mathrm{HC})$ fragments of IgG were fully separated into two distinct IEF bands. Samples were concurrently compared against capillary IEF (cIEF) revealing SIEF's equivalent glycoprotein separation power to cIEF. However, advantages such as time, low sample requirements, cost, and in-situ sample access within protein spot libraries makes sIEF a promising tool for future glycobiology research.

\subsection{Introduction}

Glycan information can reflect up to $2 \%$ of genome encoded enzymes and inherited disorders found in glycosidases and glycosyltransferases, which are involved in glycan synthesis activation. These are directly or indirectly related to several human diseases [235-242]. For this reason, exploring glycoprotein expression is critical to understand mammalian cellular processes and as such they are key targets for pharmaceutical interventions for genetic disorders and other diseased states. Proteins modified with glycans exhibit pathologically beneficial structural and charge variations due to covalently attached oligosaccharides. Manipulating glycosylation of pharmaceutically active proteins improves molecular stability, helps regulate physicochemical and pharmacological properties, and improves pharmacokinetics via better absorption and longer circulation times [243-245]. Further, the study of glycoprotein structural variations provides insights into cell growth, immune defense, viral replication, and cell-cell adhesion $[245,246]$. The glycobiology field has adopted a mapping approach to investigate oligosaccharide influences on protein properties. Structure recognition is best identified from glycosylation sites and subsequent glycosylated functional groups that change between healthy and diseased states [247]. Knowledge of extent of glycosylation, glycoprotein structures, and their biochemical pathways 
have advanced considerably with analytical tools including conventional HPLC, MS, MALDI-TOF and CE [248-250].

Mass spectral techniques such as MS or MALDI-TOF can provide explicit identifications of glycoprotein molecular weights to infer molecular structures and thus are often applied for sequential, library-based analysis such as protein mapping and automated sample/factor dependencies in protein arrays [251-253]. These well-established and commercial available techniques offer robust and reproducible data given sufficient time and funding. These drawbacks include labor-intensive sample preparation, long analysis times, limited equipment access, and high instrumentation/run costs which prevent their accessibility and utility for preliminary screening for most glycobiological analyses. For these reasons, electrophoresis based techniques remain the primary tools employed to evaluate and monitor molecule/domain charge changes due to glycol-molecular reactions such as deamination during glycosylation or glycoprotein cleavage. Electrophoretic techniques can discern charge and 3D size differences with less resolvability and sensitivity than mass spectral techniques, but the easy operation and rapid processing times make them excellent $1^{\text {st }}$-step analysis tools to proceed higher power secondary analyses.

Among regular glycobiolgy analysis tools, chromatography based techniques such as MS or MALDI-TOF can provide explicit identifications of glycoprotein molecule structures and thus be often applied for details analysis such as protein mapping and complicate sample array analysis. Those type of techniques are well established and commercial available therefor have capability of offer robust and reproducible data. However the long-time, complicate sample preparing and processing steps, as well as the high cost prevent them to be a commonly applied technique in the beginning stage of glycobiolgy analysis. Alternatively, electrophoresis based techniques are always employed to evaluate and monitor molecule/domain charge changes due to chemical reactions such as deamination during glycosylation or glycoprotein cleavage process. Those techniques cannot offer fancy capability to collect as much details as possible during analysis, but the 
easy operation and rapid processing time make them remaining to be an excellent $1^{\text {st }}$-step analysis tools and cannot be fully replaced by chromatography based techniques.

During a biological development, oligosaccharide structures of glycoproteins could change drastically, and those changes could be associated with pathological conditions. Tracking the structural change of glycoproteins is critical in clinical therapeutic development. Immunoglobulin $\mathrm{G}(\mathrm{IgG})$ is one of marketed recombinant monoclonal antibody $(\mathrm{mAb})$ under glycoprotein category and has been widely investigated on glycoprotein developments and have been extensively analyzed via cIEF. Early IgG myeloma sera research used a silica capillary and cathodic mobilization to resolve myeloma $\operatorname{lgG}$, a mAb protein species with $10-15$ fold peak height enhancements [254]. Monoclonal antibody isoforms from healthy vs ovary and lymphatic cancer donors had similar retention times but $\alpha$-1-Acid glycoprotein peak position and peak area were utilized to quantify differences [242]. Qualitative glycoprotein detection in CIEF can be achieved via whole column clEF imaging [232, 255, 256] or clEF-SDS [257]. Whole column clEF allows charged protein isoforms to be monitored and quantified in real time, which decreases single run analysis times to $\sim 18$ min per run. SDS binds with glycan moieties to disrupt glycoprotein covalent bonds enabling conformational changes and accentuating $\mathrm{p} /$ differences, which are discernable in cIEF. Automated 2-D array/sample matrix configurations interfaced with cIEF-SDS can discern proteins with molecular weights from 14-200 kDa: Modifications include clEF-MS [258, 259] and cIEF MALDI-TOF [260, 261]. These quantitative secondary detection systems increase resolution and protein fragment identification, but lengthen run processing times, increase sample/solvent consumption, and require access to the advanced instrumentation.

As the foundational standard for $\mathrm{mAb}$ and glycobiology analyses, clEF could benefit from improvements to run times, material consumption, and instrument simplicity/accessibility. Capillary IEF processing and sample demands cost time and money in pharmaceutical research. Run sequences require 2-3 hours to 
complete column prep (rinse, surface methylcellulose polymer coating), sample injection, equilibration, $\mathrm{pH}$ gradient establishment, sample focusing, and mobilizer injection to move bands to the detector, followed by column flushing. Each injection requires 10 20 $\mu \mathrm{L}$ which accumulates material consumption. Micro fluidic/lab-ona-chip technologies have enabled miniaturized (and more accessible) IEF tools with $0.5-1$ hour shorter run times and $\sim 2$ orders of magnitude smaller sample requirements while maintaining comparable clEF resolving power [68, 262, 263].

A further miniaturized surface IEF (sIEF) technique developed in our group has decreased run times further to $10 \mathrm{~min}$ and sample consumption to $1 \mathrm{ng}$ for different protein species detection/sorting including green fluorescent protein phycoerythrin and hemoglobin variants [208,264]Surface IEF's tunable $\mathrm{pH}$ gradient range and surface accessibility to samples provides simplicity and flexibility for higher level protein/reagent dependency studies. In this paper, the sIEF technique was used to reproduce a previously demonstrated glycoprotein denaturation/conformational change study [248, 265]. sIEF was first tested for mouse IgG focusing quality, and then adapted to track extent of mouse IgG denaturation and reduction. Protein denaturing conditions were varied from 0 to $8 \mathrm{M}$ urea, while reducing condition was fixed at $25 \mathrm{mM}$ Dithioerythritol (DTT). Also denaturing only and reducing only controls were conducted for denaturing and reducing comparison. The corresponding reducing products were observed via sIEF. Further, sIEF results were compared with cIEF under identical modification conditions. This novel, new sIEF demonstrates equivalent resolving capacity supporting sIEF's potential as a $1^{\text {st }}$-stage separation tool in glycobiology research that is easily interfaced with secondary mass spectrometry tools.

\subsection{Materials and Methods}

\subsubsection{Materials}

Narrow range carrier ampholytes (Pharmalyte ${ }^{\mathrm{TM}} \mathrm{pH}$ 6.7-7.7) were purchased from GE Healthcare (Pittsburgh, PA, USA). Tetramethylethylenediamine (TEMED), glycerin and ammonium persulfate (APS, made into $15 \% \mathrm{w} / \mathrm{v}$ solution as a 
polymerization catalyst for the acrylamide/bisacrylamide solution) were obtained from PlusOne (New York, NY, USA). Phosphoric acid, sodium hydroxide, acetic acid (made into 1\% w/v with E-pure water) and methylcellulose (MC, 4000cp viscocity), acrylamide/bis-acrylamide powder (29:1 ratio), urea, Dithiothreitol (DTT, $\geq 98 \%$ ) were ordered from Sigma-Aldrich (St. Louis, MO, USA). Mouse IgG (1 $\mathrm{mg} / \mathrm{mL}$ ) was purchased from Fisher Scientific, Inc. (Fishers, IN, USA), loaded directly onto sIEF gel while operating. Futurrex negative photoresist PR1-1000A and RD-6 photoresist developer (Franklin, NJ, USA) was utilized for generating reverse mold electrode pattern according to previous research [266]. Silver epoxy (chemical, MG8331, $0.007 \Omega \cdot \mathrm{cm}$ electrical resistivity and $0.90 \mathrm{~W} \mathrm{~m}^{-1} \cdot \mathrm{K}^{-1}$ thermal conductivity) was used as packaged.

\subsection{2 sIEF Device Fabrication}

The $100 \mu \mathrm{m}$-wide spaced, $300 \mu \mathrm{m}$ apart parallel micropatterned gold electrodes were prepared on glass microscope slides via soft photolithography followed by Ebeam electro-vapor deposition as previously described Chapter 3.2.2. Electrodes were comprised of $5 \mathrm{~nm} \mathrm{Ti/95} \mathrm{nm}$ Au. Hafnium oxide $\left(\mathrm{HfO}_{2}\right)$ passivation layers were sputter deposited under identical conditions as described in Chapter 5.3.2. Silver epoxy connected gauge18 gauge copper wire to the micropatterned electrode pads (Figure 1). Polyacrylamide gel was printed into a 60- $\mu \mathrm{m}$-wide, 300$\mu \mathrm{m}$-long unpolymerized acrylamide line across Au electrodes (Bioforce Nano eNabler ${ }^{\mathrm{TM}}$ ) and then allowed to gel in-situ. Figure 1 illustrates three replicate electrode pairs on one glass chip with $\mathrm{HfO}_{2}$ passivation over the $\mathrm{Ti} / \mathrm{Au}$ electrodes. 


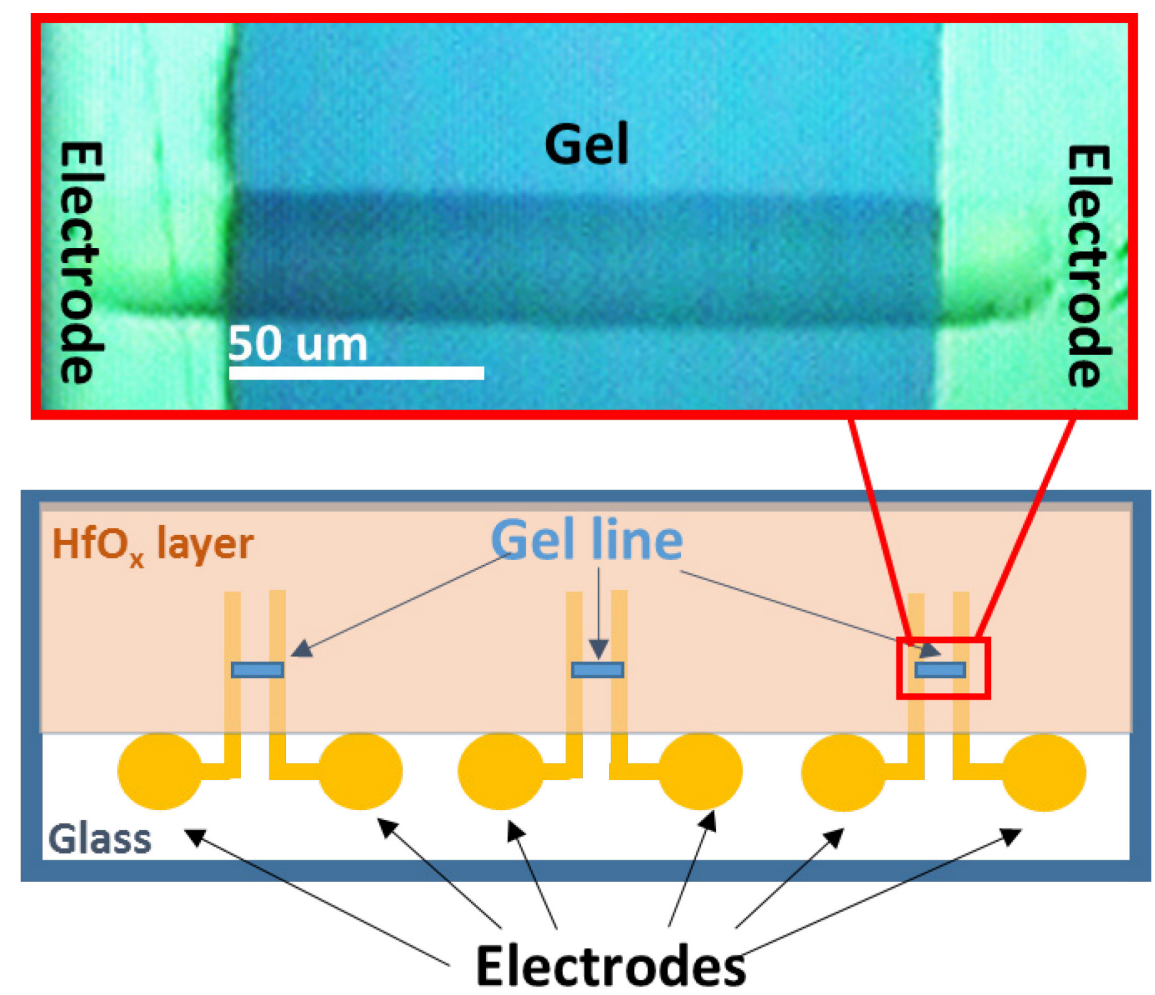

Figure 6.1 Demonstration of the electrode-Hafnium oxide passivated surface-enabled IEF (sIEF) chip. The chip was compose with three identical $\mathrm{Au} / \mathrm{Ti}$ electrode pairs, $\mathrm{HfO}_{2}$ passivation layer and printed gel lines. Each electrode pair contained functioning parts in two $100 \mu \mathrm{m}$-wide parallel lines with $300 \mu \mathrm{m}$ spare distance; $200 \mu \mathrm{m}$-radius circles were located at the end of electrode as contact pads. $\mathrm{HfO}_{2}$ passivation layer was deposited to cover functioning area of electrodes. Gel line was printed over parallel electrode area with ca. $20 \mu \mathrm{m}$ overlap on each electrode lines.

\subsubsection{Sample Preparation}

IgG, a recombinant mouse mAb, which contains two light chain (LC) and heavy chain $(\mathrm{HC})$ in its molecule, can be split into $L C$ and $\mathrm{HC}$ by partially reducing the disulfide bond that connects them [247, 267]. This was done via IgG sample denatured by urea and the reduced by DTT: IgG was firstly treated by urea at 25 ${ }^{\circ} \mathrm{C}$ overnight and then followed by DTT treatment at $65^{\circ} \mathrm{C}$ for 15 minutes. The treated IgG was directly applied on sIEF gel for experiments. Urea concentration dependency was tested under conditions of 4, 5, 6 and $8 \mathrm{M}$ urea. To investigate 
the individual effects of denaturation and reduction, two blank controls were also conducted under the conditions of only $25 \mathrm{mM}$ DTT treated lgG and only $8 \mathrm{M}$ urea treated IgG, respectively.

\subsubsection{IEF Operation}

Protein sample was directly loaded onto sIEF gel via surface patterning tool. The microscope slide was moved from the Nano eNabler ${ }^{\mathrm{TM}}$ stage to an inverted light microscope Zeiss Axiovert 200 M (Carl Zeiss Microimaging, Thornwood, NY, USA) with a 20X Neoplan objective, leads connected to Agilent 33250A function generator providing $200 \mathrm{~V} / \mathrm{cm}$ electric field through gel. Fluorescent images were recorded every 30 seconds and monitored for 10 min to observe sample focusing behaviors (fluorescent setting with excitation $485 \pm 25 \mathrm{~nm}$, emission $535 \pm 40$ ). Image contrast enhancement followed by intensity profile extraction were conducted according to the method in Chapter 5.3.3, from the middle zone (with $10 \times 300 \mu \mathrm{m}^{2}$ analysis area) of gel and output as plot via Image $\mathrm{J}$.

Control experiments were conducted to discern the $\mathrm{pH}$ gradient formation for blank control (IgG only), IgG with 5/8 M urea and $25 \mu \mathrm{M}$ DTT (control for real IgG modification process), IgG with only $25 \mu \mathrm{M}$ DTT (control for reduction environment) and IgG with only $8 \mathrm{M}$ urea (control for denature environment). which correspond to all denaturing/reducing conditions explored with the IgG. IEF markers mixture $(\mathrm{p} / 6.8,7.2$ and 7.6 mixed in $1: 1: 1 \mathrm{v} / \mathrm{v}$ ratio, with final concentrations of $0.33 \mathrm{mg} / \mathrm{mL}$ for each species) were employed to get a $\mathrm{pH}$-location fitting as illustrated in Chapter 5.4.1.

\subsubsection{Capillary IEF Operation}

All cIEF analyses were performed at room temperature $\left(25^{\circ} \mathrm{C}\right)$, the capillary was flushed with ethanol and deionized water. The protein samples together with Pharmalyte $^{\mathrm{TM}}$ and protein sample were introduced into the capillary by autosampler, focused for $20 \mathrm{~min}$ at $15 \mathrm{kV}$ and then replaced anolyte solution by acetic acid to mobilize focused species past the detector for another $10 \mathrm{~min}$. 
Capillary operations were modified to alter the direction of polarity mid-operation in order to capture proteins focusing nearest to the anode, but beyond the location of the detector. Polarity reversal enabled these proteins to be pushed back towards the cathode such that the detector could collect data. To establish a cIEF protocol, factors including separation voltage, focusing time, and protein mobilization strategies were investigated. The finalized conditions for $\mathrm{Hb}$ and $\mathrm{mAb}$ are shown in Table 8. 
Table 8 clEF run conditions for mAb system

\begin{tabular}{|l|l|}
\hline Capillary: & Neutral capillary \\
\hline Carrier ampholytes: & $2 \% \mathrm{w} / \mathrm{v}$ Pharmalyte ${ }^{\mathrm{TM}}, \mathrm{pH} 6.7-7.7$ \\
\hline Anolyte: & $0.25 \mathrm{M}$ phosphoric acid \\
\hline Catholyte: & $0.3 \mathrm{M}$ sodium hydroxide \\
\hline Mobilizer: & $0.35 \mathrm{M}$ Acetic Acid \\
\hline & $\begin{array}{l}\mathrm{p} / \text { marker } 6.8 \\
\mathrm{p} / \text { marker } 7.2 \\
\mathrm{p} / \mathrm{marker} 7.6 \\
\text { all in } 1 \mathrm{mg} / \mathrm{ml}\end{array}$ \\
\hline IEF marker: & $1 \%$ w/v methylcellulose \\
\hline EOF suppressant: & $\begin{array}{l}\text { Mouse IgG, denatured with } 0-8 \mathrm{M} \\
\text { urea, reduced by } 25 \mathrm{mM} \\
\text { dithiothreitol }\end{array}$ \\
\hline Sample: & UV-absorption at $280 \mathrm{~nm}\left(20^{\circ} \mathrm{C}\right)$ \\
\hline Detection: & 20 min, $17.5 \mathrm{kV}$ \\
\hline Focusing & $15 \mathrm{kV}$ \\
\hline Mobilization & \\
\hline & \\
\hline
\end{tabular}




\subsection{Results and Discussion}

For clinical and therapeutic developments and applications, the quality/components of glycoprotien is significantly important. In molecular level, glycoprotein quality and biological consequences can be directly linked to the structural modifications, such as domain structures and sideline positions. Therefore, it will be beneficial if those structural modifications can be characterized and monitored. IEF as a first step detection and separation tool was sensitive for structural based glycoprotein characterization due to the varied isoelectric point ( $p$ / value) during sturctual modifications. In prior work, IEF had been applied in mAb characterization for years via conventional gel IEF and capillary IEF tools [242, 268-270]. For IEF characterization of protein structural change, an artificial premodification is usually included. To deliberately modify glycoprotein in molecular level, methods including glycosylation, amino acid sequence modification, linker introduction and functional constant region removal were widely applied [271]. In the case of IgG type sample that we are studying, urea denaturing followed by dithiothreitol (DTT) partially reducing was a common method to investigate molecule domains change, it helped to individually monitor IgG domain components such as heavy chain $(\mathrm{HC})$, light chain $(\mathrm{LC})$ and crystallizable fragment (Fc) [249]. Therefore we choose the similar route for mouse IgG modification and monitored the process in sIEF system.

\subsubsection{Mouse IgG Separation via SIEF}

The sIEF operations were followed as previously reported, in short, a device with $50 \mathrm{~nm} \mathrm{HfO} 2$ passivation layer on electrodes was used in IEF separation, together with $0.5 \mathrm{w} / \mathrm{v}$ nonionic surfactant F-108 co-printing polyacrylamide gel. $\mathrm{pH}$ gradient was generated using $2 \% \mathrm{w} / \mathrm{v}$ Pharmalyte ${ }^{\mathrm{TM}}$ under $200 \mathrm{~V} / \mathrm{cm}$ electric field. Focused protein image and the corresponding image intensity profile can be seen in Figure 2. From the visual observation, mouse $\lg G$ was successfully focused into a narrow band after $200 \mathrm{~V} / \mathrm{cm}$ electric field applied and narrow pH gradient (6.7-7.7) established. Correspondingly, the focused protein was shown as a sharp peak in 
intensity profile. The $\mathrm{p} /$ value of mouse $\lg \mathrm{G}$ was estimated by comparing with a $\mathrm{pH}$-position correlation that generated using IEF markers with known $\mathrm{p} /$ values from 6.8 to 7.6, which was also detailed described in our prior work (Chapter 5.4.1). The microscope image of focused IEF markers was also shown in Figure $2 \mathrm{a}$, and the corresponding image intensity was compared with mouse $\lg G$ in Figure $2 \mathrm{~b}$. From microscope image, mouse lgG band was located at ca. $125 \mu \mathrm{m}$ from anode, this position was correlated to a equivalent $\mathrm{p} /$ value of 6.82 according to the $\mathrm{pH}$ position fitting. According to literature, the general $p /$ value of mouse IgG was in neutral $\mathrm{pH}$ range, which was around $7[268,272]$. This number was very close to the $\mathrm{p} /$ value we estimated from sIEF trail.

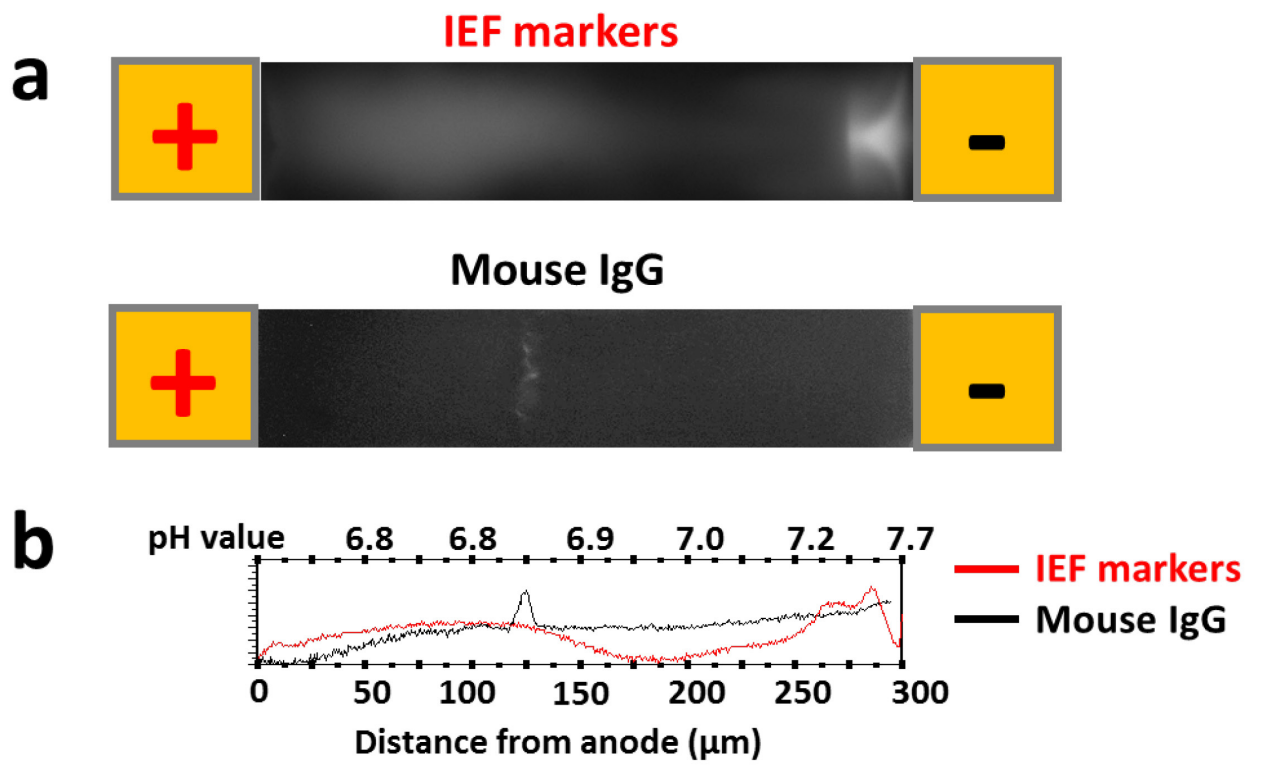

Figure 6.2 Mouse IgG sIEF separation and corresponding image intensity profile. Isoelectric focusing process was with $2 \% \mathrm{w} / \mathrm{v}$ Pharmalyte ${ }^{\mathrm{TM}}(\mathrm{pH}$ 6.7-7.7) and $200 \mathrm{~V} / \mathrm{cm}$ electric field. $1 \mathrm{mg} / \mathrm{mL}$ protein (ca. $0.9 \mathrm{ng}$ in total) was loaded directly on gel. Image was captured after 10 min focus. 


\subsubsection{Discern Protein Denaturation and Reduction Environment}

Before monitoring IgG denaturation and reduction via SIEF, it is important to understand the urea/DTT effects on $\mathrm{pH}$ gradient. For this purpose, a set of control experiments had been conducted. The corresponding $\mathrm{pH}$-location fits can be seen in Figure 3. In Figure 3a, the same denaturing and reducing environment were studied individually. Obtained $\mathrm{pH}$ gradient under $8 \mathrm{M}$ urea only condition developed in a linear way, while in the $25 \mu \mathrm{M}$ DTT only condition, the obtained pH gradient just had a slight offset toward cathode compared with blank control. This result demonstrated that urea was the main contributor to linearly developed $\mathrm{pH}$ gradient, and DTT played minor effect on $\mathrm{pH}$ gradient development. Figure $3 \mathrm{~b}$ demonstrates the $\mathrm{pH}$ gradient changes under mixed denaturing conditions of $8 \mathrm{M}$ urea and $5 \mathrm{M}$ urea with reducing condition of $25 \mu \mathrm{M}$ DTT, which is the same to the real IgG modification process. A blank control (no urea no DTT) was also incorporated in the $\mathrm{pH}$ gradient comparison. The results again showed better linearity with the presence of urea and DTT compared with the blank control. In meantime, urea concentration had impact on $\mathrm{pH}$ gradient: immensely high urea concentration in gel would compress the formed $\mathrm{pH}$ gradient toward cathode. Compared with $5 \mathrm{M}$ urea denaturing conditions, the use of $8 \mathrm{M}$ urea caused steeper $\mathrm{pH}$ gradient slope over separation space in Figure $3 \mathrm{~b}$. To make the denature reagent effect more confident, other denaturing conditions (4 M and $6 \mathrm{M}$ urea, not shown in Figure $3 \mathrm{~b}$ ) were also investigated and all the conditions and corresponding band position data were listed in Table 2. From the band positions, it is easy to see all the focused bands were consistently moved toward cathode with the increment of urea concentration. Those control experiments demonstrated the $\mathrm{pH}$ gradient would established with better linearity under IgG denature/reduce conditions, however, the over concentrated urea would lead a pH compression.

We also run IgG IEF with the same the control conditions and the microscope images and intensity profiles are shown in Figure 4 . With only reducing reagent presence, only an intact group rather than a sharp band (Figure $4 \mathrm{c}$ and f) was observed; with only denaturing reagent presence, a broad focused band was 
observed and the $\mathrm{p} /$ value of this band shifted over 7 . Those phenomena were due to the IgG denaturing-reducing sequence: the artificial IgG structural modification followed denaturing-reducing sequence, protein molecules were firstly unfolded by denaturing reagent and then became partially reduced by reducing reagent. With only $8 \mathrm{M}$ urea, IgG was not reduced but the high urea concentration made a compressed $\mathrm{pH}$ gradient, therefore a band broadening and shift could be observed; with only DTT, folded IgG molecules were only reduced in very low level and the internal bonding was not released, that explained the intact band formed after focusing. In all, reducing reagent did not play a major role within the $\mathrm{pH}$ gradient during IEF process, while the concentration of denaturing reagent affected $\mathrm{pH}$ gradient dynamically.
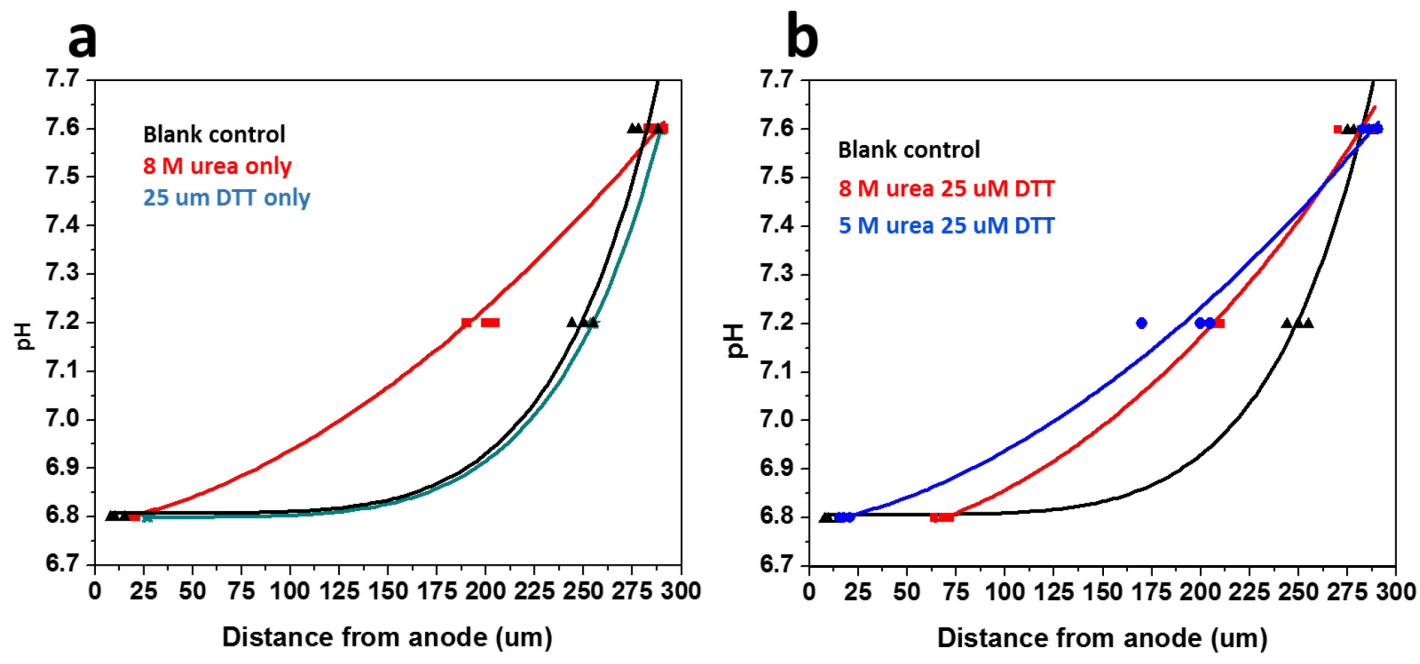

Figure $6.3 \mathrm{pH}$ gradient fits under different environment controls. a) A comparison of $\mathrm{pH}$ gradients under blank control, $8 \mathrm{M}$ urea only and $25 \mu \mathrm{M}$ DTT only. The most linear $\mathrm{pH}$ gradient was obtained with $8 \mathrm{M}$ urea control. With only $25 \mu \mathrm{M}$ DTT, $\mathrm{pH}$ gradient showed tiny drift toward cathode comparing with blank control, b) A comparison of $\mathrm{pH}$ gradients under blank control, $8 \mathrm{M}$ urea $25 \mu \mathrm{M}$ DTT and $5 \mathrm{M}$ urea $25 \mu \mathrm{M}$ DTT. Consistent with urea/DTT only control, the introducing of urea helped $\mathrm{pH}$ gradient to maintain linear tendency, while the combination of over concentrated urea concentration $(8 \mathrm{M})$ and DTT made $\mathrm{pH}$ gradient compressed again. All IEF experiments were operated with $2 \% \mathrm{w} / \mathrm{v}$ Pharmalyte $^{\mathrm{TM}}(\mathrm{pH}$ 6.7-7.7) and 200 $\mathrm{V} / \mathrm{cm}$ electric field. 
Table 9 Focused IgG/lgG fragments locations under different denaturing and reducing conditions

\begin{tabular}{|c|c|}
\hline Conditions & $\begin{array}{c}\text { Averaged band } \\
\text { positions ( } \mu \mathrm{m} \text { from } \\
\text { anode) }\end{array}$ \\
\hline No treatment & $138.4 \pm 10.1$ \\
\hline 0 M urea & $130.4 \pm 1.8$ \\
\hline 25 mM DTT & LC $155.2 \pm 21.8$ \\
\hline 4 M urea & HC $201.0 \pm 9.5$ \\
\hline 5 M urea 25 mM DTT & LC $157.2 \pm 23.8$ \\
HC $200.0 \pm 8.5$
\end{tabular}

Since all the $\mathrm{pH}$ gradient control experiments were operated under IgG denature and reduce environment, it is necessary to have a control experiment that studies reduce environment individually, so the effect on $\mathrm{pH}$ gradient from DTT could be excluded. An $8 \mathrm{M}$ urea only and a $25 \mu \mathrm{M}$ DTT only experiments were run separately as control for individual denature and reduce environment. The fitted pH-location curves are shown in Figure 5. Similar to the urea+DTT control, obtained $\mathrm{pH}$ gradient under $8 \mathrm{M}$ urea only condition developed linearly, while in the $25 \mu \mathrm{M}$ DTT only condition, the obtained $\mathrm{pH}$ gradient did not have much offset compared with no urea no DTT control. This result demonstrated the minor effect from DTT on $\mathrm{pH}$ gradient development. We also run IgG IEF on the control conditions and the microscope images and intensity profiles are shown in Figure 
6.4. As describe in previous section, the artificial IgG structural modification followed denaturing-reducing sequence, protein molecules were unfolded by denaturing reagent first and then became partially reduced by reducing reagent. With only reducing reagent presence, only the surface of protein was reduced and focused as an intact group rather than a sharp band (Figure $6.4 \mathrm{c}$ and f); with only denaturing reagent presence, also a broad focused band was observed and the $\mathrm{p} /$ value of this band shifted over 7 . It could be the unusual protein structure change under extreme denaturing condition ( $8 \mathrm{M}$ urea). As a conclusion, reducing reagent did not play a major role within the $\mathrm{pH}$ gradient during IEF process, while the concentration of denaturing reagent affected $\mathrm{pH}$ gradient dynamically.

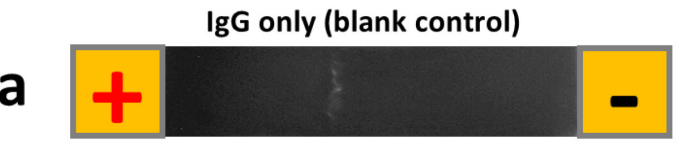

$8 \mathrm{M}$ urea (unfolding only control)

b
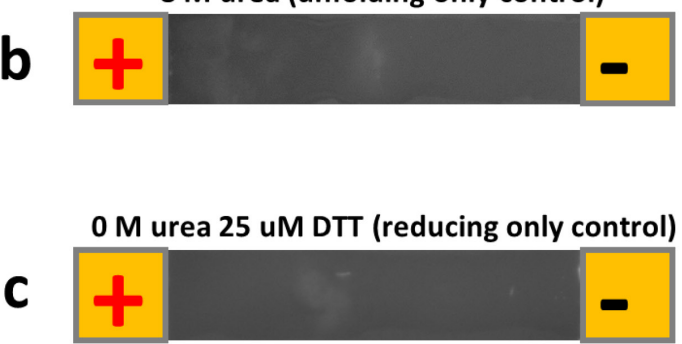
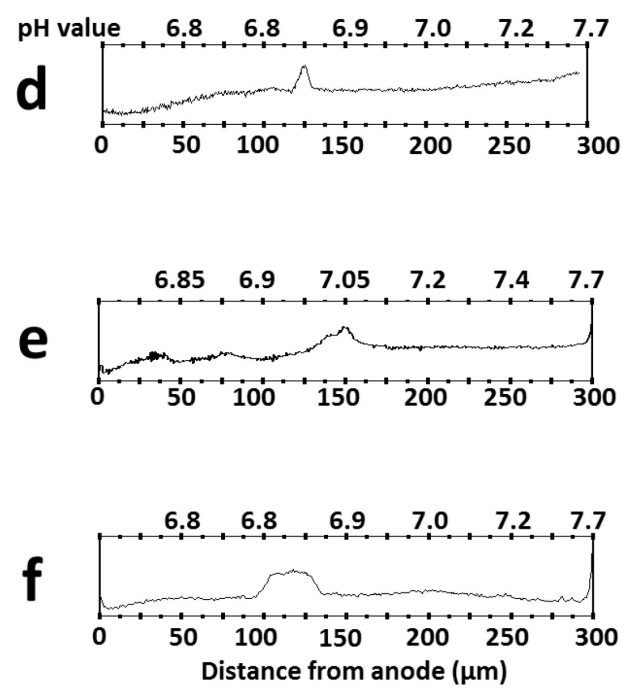

Figure 6.4 Denature and reduce environment control of mouse IgG. a-c) microscope images of blank control, unfolding only control and reducing only control, respectively. d-f) Image intensity and $\mathrm{pH}$ profiles of IEF images. Without any denaturing/reducing agent, IgG was focused into a sharp band. Without only reducing agent DTT, an intact band was observed in the experiment. With only reducing agent urea, IgG focused band was broaden, also the band shifted toward cathode. None of those control experiments showed a separation of LC and HC. This tendency was consistent with the $\mathrm{pH}$ gradient control. All IEF experiments were operated with $2 \% \mathrm{w} / \mathrm{v}$ Pharmalyte $^{\mathrm{TM}}(\mathrm{pH}$ 6.7-7.7) and $200 \mathrm{~V} / \mathrm{cm}$ electric field. 


\subsubsection{Protein Isoelectric Focusing under Mixed Denaturing and Reducing Conditions}

As mentioned previously, urea-DTT treatment was adapted to mouse IgG for sIEF sampling. Denature control were varied by urea concentration at 4 8 $\mathrm{M}$ and reduce condition was fixed using $25 \mu \mathrm{M}$ of DTT. Because IgG would appeared as intact sample under native condition ( $0 \mathrm{M}$ urea), this condition was also in urea concentration list. The microscope images after $\lg G$ focused and a corresponding intensity profiles are shown in Figure 6.5. Under native condition (0 M urea), DTT reduced protein shown as an intact piece in microscope image and a flat head peak was correspondingly found in intensity profile (Figure $6.5 \mathrm{a}$ and f). As urea concentration increased, the intact piece became unleashed and the fragments became redistributed along the SIEF gel (Figure $6.5 \mathrm{~b}$ and $\mathrm{g}$ ). Once urea concentration reached to $5 \mathrm{M}$, two separated bands was observed in gel line (Figure $6.5 \mathrm{c}$ and $\mathrm{h}$ ), due to the separation of LC and HC fragments. As urea concentration increased to $6 \mathrm{M}$, the focusing became more integrated (Figure 6.5 $\mathrm{d}$ and $\mathrm{i}$ ). The further increasing of urea concentration made LC and $\mathrm{HC}$ band focused being more integrated. However, the urea concentration could not be infinitely increased. There is a solubility of urea, also the high denature concentration can interrupt the $\mathrm{pH}$ gradient establishment according to previous studies [164]. In our experiment, maximum urea concentration was $8 \mathrm{M}$, focused images and intensity profiles can be seen in Figure $6.5 \mathrm{e}$ and j. A slightly bands movement toward cathode was observed under $8 \mathrm{M}$ urea concentration, which implicated a $\mathrm{pH}$ gradient shift. This observation was consistent with both the $\mathrm{pH}$ gradient change in previous section and the description from literatures. 

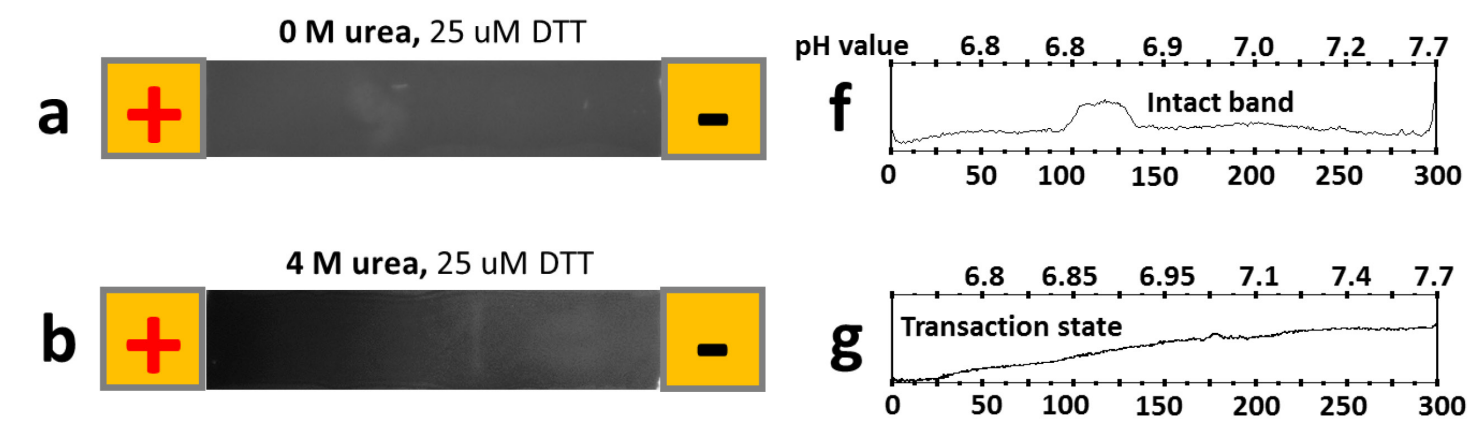

C
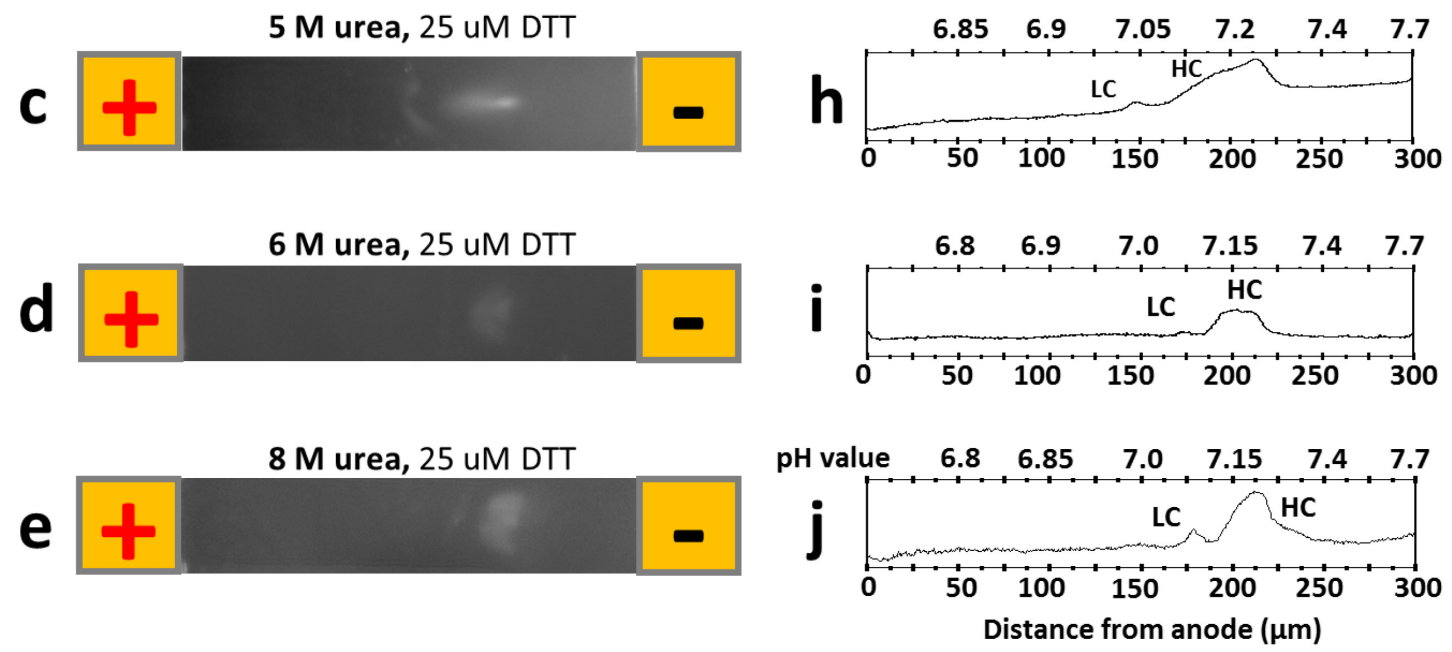

Figure 6.5 Denature condition control of mouse IgG reduction. a-e) microscope images of protein reduction products at the denature condition of $0 \mathrm{M}, 4 \mathrm{M}, 5 \mathrm{M}, 6 \mathrm{M}$ and $8 \mathrm{M}$ urea environment, respectively. $\mathrm{f}-\mathrm{j}$ ) Image intensity and $\mathrm{pH}$ profiles of IEF images. With $0 \mathrm{M}$ urea, the partially reduced IgG was focused as an intact band, as urea concentration increased, the intact protein became unfolded. A separation of $L C$ and $\mathrm{HC}$ started at $5 \mathrm{M}$ urea concentration and the separation level became better with increased urea concentration. Also the focused bands revealed shift toward cathode with $6 \mathrm{M}$ and $8 \mathrm{M}$ due to the $\mathrm{pH}$ gradient compression. IEF was operated with $2 \% \mathrm{w} / \mathrm{v}$ Pharmalyte ${ }^{\mathrm{TM}}(\mathrm{pH}$ 6.7-7.7) and $200 \mathrm{~V} / \mathrm{cm}$ electric field. Reduction condition was fixed at $25 \mu \mathrm{M}$ DTT. Corresponding $\mathrm{pH}$-location differences at each conditions were also labeled in intensity profiles.

\subsection{4 sIEF Focusing Verification with cIEF}

A comparison between sIEF and cIEF results was conducted for the same sample preparation and treatment. For sIEF, microscope images of focused mAb species were captured and a corresponding intensity profile was obtained to identify gel locations of the focused species. A separate $\mathrm{pH}$-band calibration curve was also 
fitted using fluorescent IEF markers 6.8, 7.2, and 7.6. For CIEF, the same IEF markers were used as internal standards and mixed directly with the IgG samples; this enabled the estimation of the relative $p /$ values of IgG reducing species. As first step, untreated IgG focusing under sIEF and CIEF were compared. The combined sIEF-clEF comparisons are shown below in Figure 6.6a. In both of the two IEF techniques, $\mathrm{pH}$ gradient was successfully formed and IgG species were successfully focused. By pulling out the $\mathrm{pH}$ profile, the three point of $\mathrm{pH}$ gradient (6.8 7.2 and 7.6) are relatively consistent. The focused IgG shown as a standalone peak at the location of $125 \mathrm{um}$ from anode, which corresponding to $\mathrm{pH}$ $6.81 \pm 0.003$ in sIEF, and the IgG peak is located in the middle of $\mathrm{pH} 6.8$ and 7.2 in CIEF. However, the protein was shown as a scattered pattern rather than fully focused in ClEF.

Secondly, Figure $6.6 \mathrm{~b}$ shows sIEF and cIEF results of IgG treated under $8 \mathrm{M}$ urea and $25 \mu \mathrm{m}$ DTT. Successful $\mathrm{pH}$ gradient establishment and IgG focusing were observed in both techniques. The ends of the $\mathrm{pH}$ gradient (6.8 and 7.6) were consistent. However, in clEF's pH 7.2-7.6 region, the $\mathrm{pH}$ gradient is narrower than sIEF implicating that sIEF experienced less $\mathrm{pH}$ compression than clEF. Compression translates into loss of resolution for protein focusing. Sample focusing locations in SIEF are in the 7.0-7.3 region; while the locations in CIEF are in 6.9-7.2 region. These mappings suggest a $\sim 0.1 \mathrm{pH}$ inconsistency between sIEF and CIEF. The reduced products from IgG treatment, light chain (LC) and heavy chain (HC) are located at 157 um and 200 um from anode in sIEF, which correlated 
to $\mathrm{pH} 7.03 \pm 0.083$ and $\mathrm{pH} 7.19 \pm 0.036$, respectively. While in clEF all the reduced products are located in the middle of $\mathrm{pH} 6.8$ and 7.2 , which roughly at 7.0.
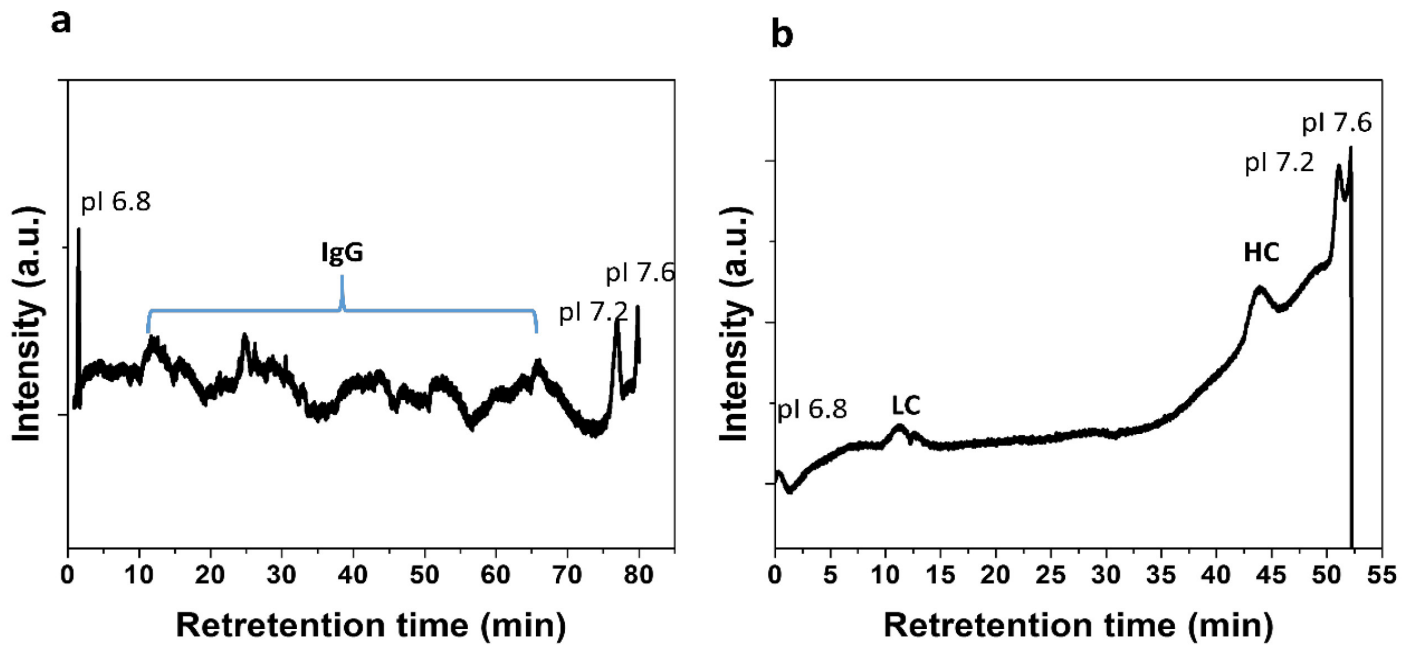

Figure $6.6 \mathrm{clEF}$ run results of IgG in different conditions. a) Untreated IgG mixed with pl markers 6.8, 7.2 and 7.6. IgG molecule was shown as unfocused group. b) IgG treated with $8 \mathrm{M}$ urea and $25 \mu \mathrm{M}$ DTT and mixed with pl markers $6.8,7.2$ and 7.6 . $\mathrm{LC}$ and $\mathrm{HC}$ segments were successfully separated at the location where $\mathrm{pH}$ was approximately 7 .

\subsection{Conclusions}

This work explored miniaturized surface isoelectric focusing (sIEF) for artificially glycoprotein molecular modification monitoring. A narrow range $\mathrm{pH}$ gradient was established within electrode passivated SIEF chip to resolve a selected glycoprotein spices-mouse IgG. As a visualized IEF technique, sIEF borrowed conventional whole column imaging cIEF concept, proteins focusing in transparent gel media was directly monitored under microscope. Also the entire focusing process only required less than 10 minutes. The resolving capability was examined with untreated IgG sample and later the denaturing-partially reducing treated IgG was tested. The focused IgG protein band was recorded using microscope, and the corresponding isoelectric point was estimated to be 6.82 , according to a position-pH correlation that developed from fluorescent IEF markers. The denaturing-partially reducing operation was controlled using urea and dithiothreitol 
(DTT). IgG was denatured with urea and then reduced by DTT. The denaturing level was controlled by varying urea concentration from native $(0 M)$ to saturated (8 M). DTT concentration was fixed at $25 \mu \mathrm{M}$ to reduce IgG partially. Results illustrated that $\lg G$ reducing products were shown as intact band at native condition due to the unfolded molecule structure. As denaturing reached to higher level, the IgG became better unfolded and two of the main reduce product light chain (LC) and heavy chain $(\mathrm{HC})$ were cleanly separated. However the focused band experienced a drift toward cathode under saturated urea condition. To rule out the contribution of DTT for protein band cathodic drift, a blank control that only included varied urea concentration was conducted and a focus position-pH correlation was produced using IEF markers. More severe $\mathrm{pH}$ gradient compression was observed under higher urea concentration. In the meantime, the $\mathrm{pH}$ profile from the condition with only $25 \mu \mathrm{M}$ DTT revealed negligible change compared with no DTT no urea control. This suggested urea was the only active factor to interrupt $\mathrm{pH}$ gradient during isoelectric focusing in sIEF system.

Additionally, the denaturing-partial reducing results were also verified with commercial clEF. The observations demonstrated a great consistency between sIEF and CIEF, which suggested sIEF was able to successfully monitor/detect the IgG molecular changes. Although clEF remains to be an ideal tool for the highest resolution requirement, sIEF is more adaptive as $1^{\text {st }}$ step screening tool for time sensitive, space confined, portable, screening demands. It has sufficient resolving power and versatility to be integrated with advanced post-analyses tools such as MS and MALDI-TOF. 


\section{Future Work}

In this dissertation, the new sIEF approach to small volume protein separations has been systematically explored from concept design to real world applications. sIEF offers the potential for simpler, cheaper, quicker, and time sensitive analysis by operating 100 times smaller than previous IEF techniques. The main objective of this dissertation was to establish a new concept and investigate its feasibility. Due to $\mathrm{PhD}$ study cycle time constrains, there could be some details that have not been fully covered and are listed in the following paragraphs of this chapter.

\subsection{Quantifying Electroosmotic Flow (EOF) on Hafnium Oxide Surfaces for Surface IsoElectric Focusing (sIEF)}

Electroosmotic flow (EOF) is a common phenomenon in microfluidic system. As a dynamic force in fluidics, EOF possibly exists in sIEF due to the solution that is introduced from sampling. As a mass transport force, EOF will play as a counterfocusing factor in a sIEF system, and causes disturbances during focusing. This phenomenon was experienced in our preliminary results. According to previously reported cIEF work, the most common method to reduce EOF is by coating the channel surfaces with polymers $[4,206,207,228,230]$. To better understand these forces, a systematic investigation of EOF-suppression should be conducted. To stimulate the conventional coatings method, a thin layer of hafnium oxide, which was applied in this dissertation, will be used for sIEF device surface coating on the glass slide. The EOF mobility behavior in that hafnium oxide coated device will be measured using the current monitoring method [273]. Proposed device setting is shown in Figure 7.1. A PDMS layer with a micro fluidic channel will be sealed together with halfnium oxide on the top and bottom. As a comparison, EOF will also be tested in a similar device having been sealed together with glass on the top and bottom. 


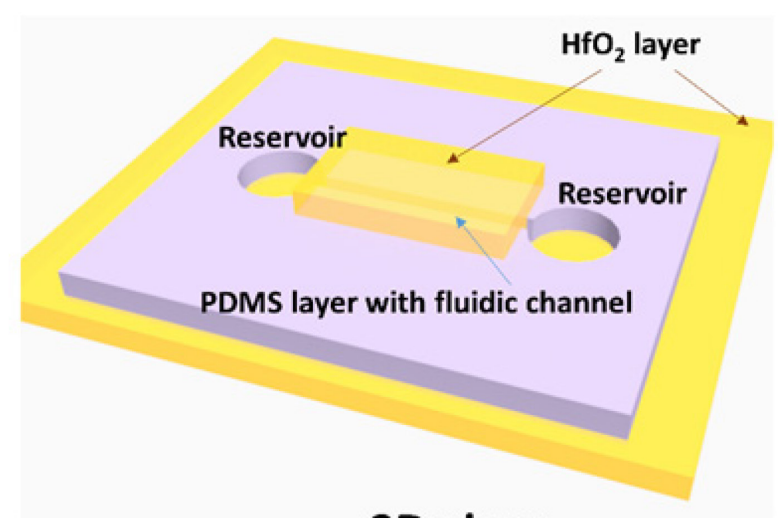

3D view

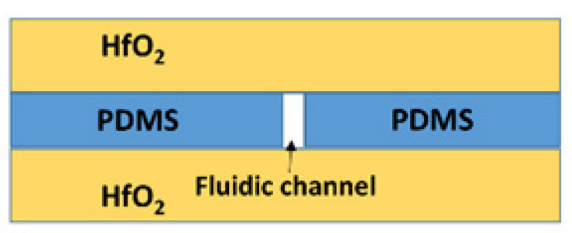

Side view

Figure 7.1 EOF test device design with $\mathrm{HfO}_{2}$ coating

\subsection{Further Exploration of the Device Design.}

In this dissertation, sIEF device design and separation space were fixed based on the preliminary optimization; however, there is still room to utilize creativity on device designs. As the first priority, varied IEF separation space should be considered. The idea to address this is a tapered electrode design, which is shown in Figure 7.1. Due to the feasibility of Nano eNabler, a gel line can be created in any position between two electrodes and therefore the separation distance can be varied along the tapered electrodes. Secondly, incorporation of a sensing electrode array under the IEF gel, which can assist resolving focused protein bands. Confined by optical limitations, some proteins are hard to be imaged in sIEF with good resolution, therefore, a spatially resolvable detection via an array of micro-patterned sensing electrodes underneath the sIEF gel could be a solution for this is shown of discerning proteins according to their focused locations. A proposed design for this is shown in Figure 7.2.

The composition/structure of the gel can be considered as an independent variable in the future sIEF work. As illustrated in Chapter 2, the pore size of polyacrylamide gel is controlled by acrylamide/bis ratio. To achieve the best separation efficiency, gel pore size should be tuned according to the target protein size. Information 
regarding the dependency of gel pore-sample size should be included in future sIEF work.
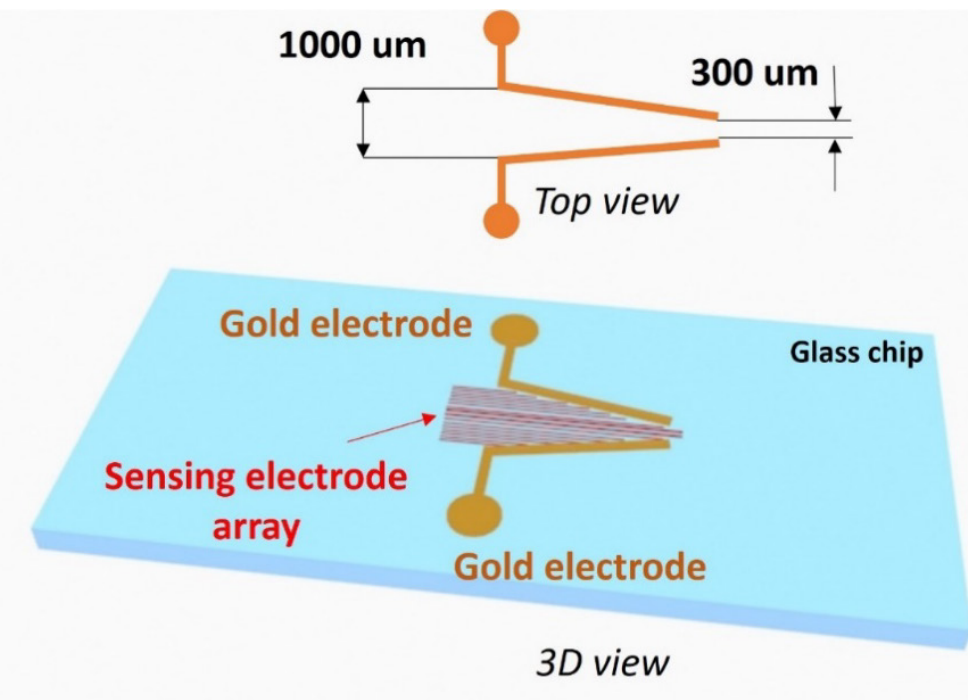

Figure 7.2 3-D view of tapered electrode design and a series of parallel sensing electrode lines ca.10 microns across and spaced ca. 10-20 microns apart located between the two sIEF working electrodes. Local capacitance of the gels can be determined by sequentially applying DC or AC potentials to pairs of sensing electrodes.

\subsection{Image Detection for Band Identification}

Automatic data processing is desirable in any IEF work. In sIEF, the image resolution of focused protein band is due to the protein type. The colorless or undyed proteins usually bring difficulties for image detection; therefore, automatic image processing will be crucial for post focusing analysis. As a possible solution, a widely used scale-invariant feature transform (SIFT) method could be adapted to sIEF. This technique has functions including detection of scale-space extrema, accurate keypoint localization, orientation assignment, and specifically in sIEF local image descriptors [274]. SIFT can be used to detect the focused band from microscope images. To process a sIEF image, a Matlab code can be created by 
the following the sequence: 1) select a reference feature from detection area, 2) define a similarity based on reference feature, 3) define a similarity threshold, 4) start a rule-out calculation, 5) scan the entire image, and output the test image analysis result. A demonstration can be seen in Figure 7.3. A reference image is given at the beginning, and a set of criteria for the region of interest will be preinput into the computer. Once a testing image has been loaded, the computer will automatically scan the image and locate the region with the best similarity to the reference image. By setting up this similarity threshold, it is possible to pull out the focused band directly from loaded images. With the help from the SIFT code, the sIEF post image analysis will be more robust and have a better confidence interval.
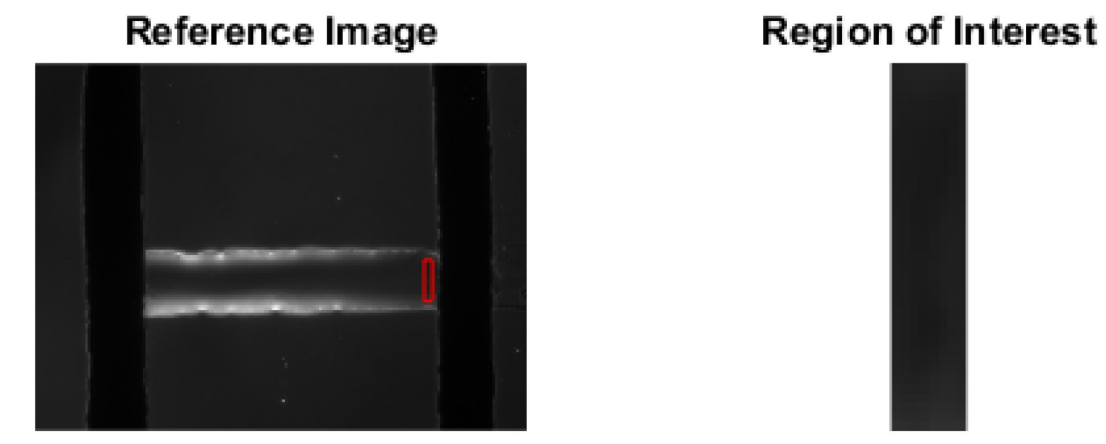

Testing Image

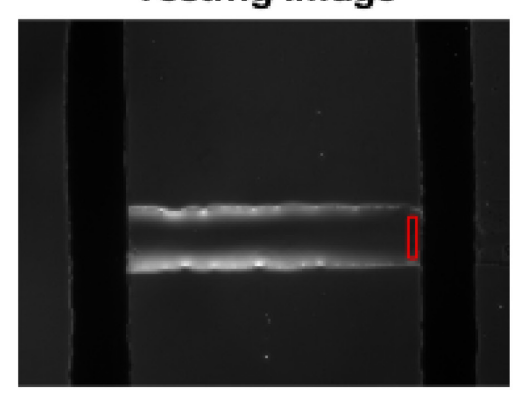

Band detection

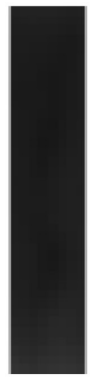

Figure 7.3 Interested area image detection demo. A reference image was pre-loaded into Matlab with the interested area circled out by a red color, as shown in the top row. The testing image was then loaded into Matlab, and the area with the best similarity was detected using the rule of interested area feature. The results were then outputted as shown in the bottom row. 


\section{Conclusions}

\subsection{Introduction}

Isoelectric focusing techniques have been developed in a miniaturized direction, to achieve low sample requirements, portable, economic friendly and easy access. The separation space have been reduced from the centimeter to the millimeter scale or even smaller. In the meantime the development of advanced micromanipulation techniques such as AFM and FEMTO play an important role in moving toward IEF miniaturization. Further, exploration of IEF miniaturization will be a challenging, but also a remarkable experience, to test the lowest space limit of IEF design and fabrication based on conventional and new born technologies. The surface enabled isoelectric focusing (SIEF) work presented in this dissertation is a creation designed combining old and new microfabrication techniques. The conventional metal deposition method is a convenient way to make a planar metal layer; therefore, it is being applied in sIEF for electrode fabrication. The nanometer scale electrode thickness offers plenty of flexibility for IEF separation space distribution design. A novel FEMTO based surface printing technique brings an opportunity for nano-scale IEF media patterning, working with practical planar electrode design, a sIEF device can be miniaturized in magnitude compared with the currently existing IEF system. During device fabrication, different parameters including printing humidity, surface hydrophilicity, IEF media loading method and sIEF gel polymerization have been considered and optimized. This novel method for sIEF device fabrication brings with it the opportunity to explore the miniaturization limit of IEF techniques.

Carrier ampholytes (CAs) have been proven to be an efficient way of establishing continuous $\mathrm{pH}$ gradient. Also the high commercialization level makes $\mathrm{CA}$ type $\mathrm{pH}$ gradient robust and highly reproducible. Therefore this method has been grafted to the sIEF device in this work. The established $\mathrm{pH}$ gradient has been successfully characterized by $\mathrm{pH}$ sensitive dyes, with the $\mathrm{pH}$ gradient range tunable by selecting different commercial CA products. To verify the resolving power of sIEF in protein separation and detection, different protein systems including GFP-PE 
mixture, hemoglobin variants and mouse lgG partially reduced products have been tested as samples. Results demonstrated that sIEF has adequate resolving power as well as highly adaptive characteristics for time sensitive, space confined, and portable, screening demands; therefore, sIEF is a good complementary technique to currently existing IEF.

\subsection{SIEF Device Design and Fabrication}

The two main components of a miniaturized SIEF device, electrodes and separation gel media, were fabricated via metal deposition and a nano-printer, respectively. For metal deposition, a uniform layer with controllable deposition rate could be achieved by using commercial sputter or an electron-beam depositor; therefore, sIEF device optimization was focused on nano-printing operations, which was first time adapted for IEF purposes. Printing parameters including methods of sample loading, surface hydrophobic/hydrophilic property, printing humidity and acrylamide gelation were explored, were investigated to ensure a nice line shape with good reproducibility. The final recipe was determined to be SPT front loading, 10-minute UV-ozone recovery of surface hydrophilicity, 10\% 20\% relative printing humidity, and the use of $15 \% \mathrm{w} / \mathrm{v}$ APS with 3 hours of polymerization. With these optimized printing parameters, a straight acrylamide/bis gel line sized $300 \mu \mathrm{m}(\mathrm{L}) \times 60 \mu \mathrm{m}(\mathrm{W}) \times 35 \mu \mathrm{m}(\mathrm{H})$ could be successfully fabricated, which then prepares the ready-to-go platform for the sIEF operation.

\subsection{SIEF with Broad Range pH Gradient and Protein Resolving Test}

Using the produced sIEF device with separation space at a scale of $100 \mu \mathrm{m}$, a CA type $\mathrm{pH}$ gradient was ready to be established. The commercially available CA product Pharmalyte ${ }^{\mathrm{TM}}$, with broad $\mathrm{pH}$ ranging 3 to 10 , was applied to generate a continuous $\mathrm{pH}$ gradient with the electric field turned on. This $\mathrm{pH}$ gradient was verified via a gradually decreasing intensity ratio of $\mathrm{pH}$ sensitive and $\mathrm{pH}$ insensitive fluorescent dyes along the separation gel space. Furthermore, a mixture of GFP and R-PE protein samples were tested with an extremely small loading amount of $0.9 \mathrm{ng}$. The focusing results visually showed GFP and R-PE were separated and focused to $\mathrm{pH} \sim 4$ and $\mathrm{pH} \sim 6$, respectively, and was consistent with their $\mathrm{p} /$ values. 
Electric field strengths and carrier ampholyte concentrations were investigated to determine the optimized experimental conditions of $2 \% \mathrm{w} / \mathrm{v}$ Pharmalyte $^{\mathrm{TM}}$ and 200 $\mathrm{V} / \mathrm{cm}$.

A $\mathrm{pH}$ gradient drift/compression after $\mathrm{pH}$ gradient establishment, which is a common issue that involved in conventional IEF, was also observed in sIEF. To alleviate this problem, a nonionic surfactant was co-printed within a gel with the aim of reducing the physical-chemical interactions in the sample-gel-chip interfaces, and control the EOF on the chip surface. Experimental results revealed improvements of 3-7 fold in protein separation efficiencies and in the sIEF resolving capability, which will eventually improve the performance of sIEF.

In summary, sIEF experimental results demonstrated that dimension reduction in gel length not only enabled a 10 -fold reduction in power requirements but also offered a 100 -fold less sample requirement. Additionally, the sIEF chip could be easily cleaned for reusability in more than 50 runs. This novel, surface enabled IEF method, will enable rapid construction of customizable sIEF gels. The surface geometry allow for easily accessible spot picking for secondary protein analysis; therefore, it can be integrated into protein array libraries for specialized and/or orthogonal separations.

\subsection{SIEF with Narrow Range pH Gradient and Protein Resolving Test}

Similar to traditional CA type $\mathrm{pH}$ gradient IEF work, sIEF also has a tunable $\mathrm{pH}$ gradient by using CA in different ranges. This capability was verified in this dissertation. In Chapter 5, sIEF for narrow $\mathrm{pH}$ range Pharmalyte ${ }^{\mathrm{TM}}$ was explored as an extension of previously broad $\mathrm{pH}$ range sIEF work. A narrow range $\mathrm{pH}$ gradient from 6.7-7.7 was successfully established between two microfabricated sIEF electrodes with the use of commercial 6.7-7.7 Pharmalyte ${ }^{T M}$. Fluorescent IEF markers with $\mathrm{p} /$ values of $6.8,7.2$ and 7.6 were utilized to find a $\mathrm{pH}$-gel position correlation. These successfully generated and imaged $\mathrm{pH}$ gradients suggest that other narrowly confined $\mathrm{pH}$ ranges could be sustained in an SIEF gel by specifying the carrier ampholyte mixture for desired $\mathrm{pH}$ values. 
Resloving power was later examined with a highly relevant medical diagnostic system of three hemoglobin variants, $\mathrm{HbA}, \mathrm{F}$, and $\mathrm{S}$, with hard to resolve $0.2 \mathrm{p} /$ differences. The hemoglobin variants were successfully separated and identified within the narrow range $\mathrm{pH}$ gradients, exhibiting $12-15 \mu \mathrm{m}$ spatial separation of $\mathrm{Hb}$ bands in less than 10 minutes and sample volumes as low as $2 \mathrm{~nL}$.

With the aim to improve sIEF resolving power, a thin dielectric $\mathrm{HfO}_{2}$ film was deposited between the electrode surfaces and the aqueous gel media. This prevented direct contact, and reduced the electrochemical reactions at the electrode surfaces to yield a more broadly distributed $\mathrm{pH}$ gradient and improved protein band shapes. The contribution insulation layer suggested it could be extended to any IEF electrode to suppress electrolysis. Additionally, the non-ionic surfactant F-108 was co-printed with the gel to improve protein/gel/surface interactions, and it successfully demonstrated improvements to the focused band shapes. Overall, the most effective $\mathrm{Hb}$ variant separations were achieved with a combination of $\mathrm{HfO}_{2}$ surface passivation, $0.5 \% \mathrm{w} / \mathrm{v} \mathrm{F-108}$, and $2 \% \mathrm{w} / \mathrm{v}$ Pharmalyte ${ }^{\mathrm{TM}}$. These parameters could be futher utilized in the future narrow range sIEF work.

In summary, sIEF performance places it alongside micro isoelectric focsing ( $\mu \mid E F)$ for time sensitive, space confined and portablescreening demands including rapidly discerning structural variations of proteins for medical diagnostics and pharmaceutical synthesis. Additional attributes of sIEF beyond $\mu \mathrm{IEF}$ include easier surface access, simpler device fabrication and device reusability up to $\sim 50$ times. SIEF has sufficient resolving power and versatility to be adapted as a prefractionation tool for portable and fast analysis requirements that are highly suitable for most first dimension separations. SIEF also can be further integrated with post-analysis tools for orthogonal separations and/or other more advanced biological analysis of molecules. 


\subsection{Glycoprotein Modification Monitoring by sIEF with Narrow Range pH}

Following real protein tests under different $\mathrm{pH}$ gradients, the flexibility of sIEF being used for real world applications needed to be examined. Glycoprotein is an important protein subcategory for pharmacological applications because the structural variations of glycoproteins provides insight into cell growth, immune defense, viral replication, and cell-cell adhesion. Technologies that adapt to time sensitive, low sample stock and portable analyses scenarios are still inadequate in the modern world. In chapter 6 of this dissertation, sIEF was explored for artificial glycoprotein molecular modification monitoring. Narrow range $\mathrm{pH}$ gradient once again was established within electrode passivated sIEF chip to resolve a selected glycoprotein-mouse $\mathrm{IgG}$, and a position- $\mathrm{pH}$ correlation was developed from fluorescent IEF markers. To monitor IgG molecular modifications, an artifical denaturing-partially reducing operation was conducted using urea and dithiothreitol (DTT). This resulted in the development of IgG reducing products were successfully observed under microscope using sIEF devices. The higher the denaturation level, the better separation of the main products light chain (LC) and heavy chain $(\mathrm{HC})$. To determine the effect of DTT and urea on pH gradient, a blank control that included only variations of urea concentrations was conducted, and a focus position-pH correlation was also produced using IEF markers. More severe $\mathrm{pH}$ gradient compression was observed under higher urea concentrations. In the meantime, the $\mathrm{pH}$ profile having only $25 \mu \mathrm{M}$ DTT revealed negligible change compared with no DTT no urea control. This suggested urea was the only active factor that interrupted $\mathrm{pH}$ gradient during IEF in sIEF system.

Additionally, the denaturing-partial reducing results were also verified with commercial clEF. The observations demonstrated a great consistency between sIEF and CIEF, which suggested SIEF was able to successfully monitor/detect the IgG molecular changes. As mentioned in the beginning, sIEF is more adaptive as the 1st step screening tool for time sensitive, space confined, and portable screening demands. The high versatility of sIEF also makes it capable of being integrated with advanced post-analyses tools. Therefore, sIEF is a strong 
candidate in becoming the next generation of 1st step screening tool in protein analyses. 


\section{Appendix A: Microfabrication Demonstration and Standard Operating Procedure}

Microfabrication applies well established technologies for substrate processing and manufacturing. In this dissertation, the main purpose of microfabrication was to electroplate metals and metal oxides in a pre-designed pattern on a substrate, in order to get micro-sized electrodes for a lab-on-a-chip device. The general process flow includes spin coating (distribute a thin layer of photoresist uniformly on a substrate), lithography (crosslink/bond photoresist under UV energy), development (further crosslink/bond photoresist and remove the residuals to create designed features on photoresist layer), metal deposition (create metal layer with replicated photoresist pattern), and lift-off (remove metal and photoresist residuals and obtain the final device). This process flow is demonstrated in Figure AP1.

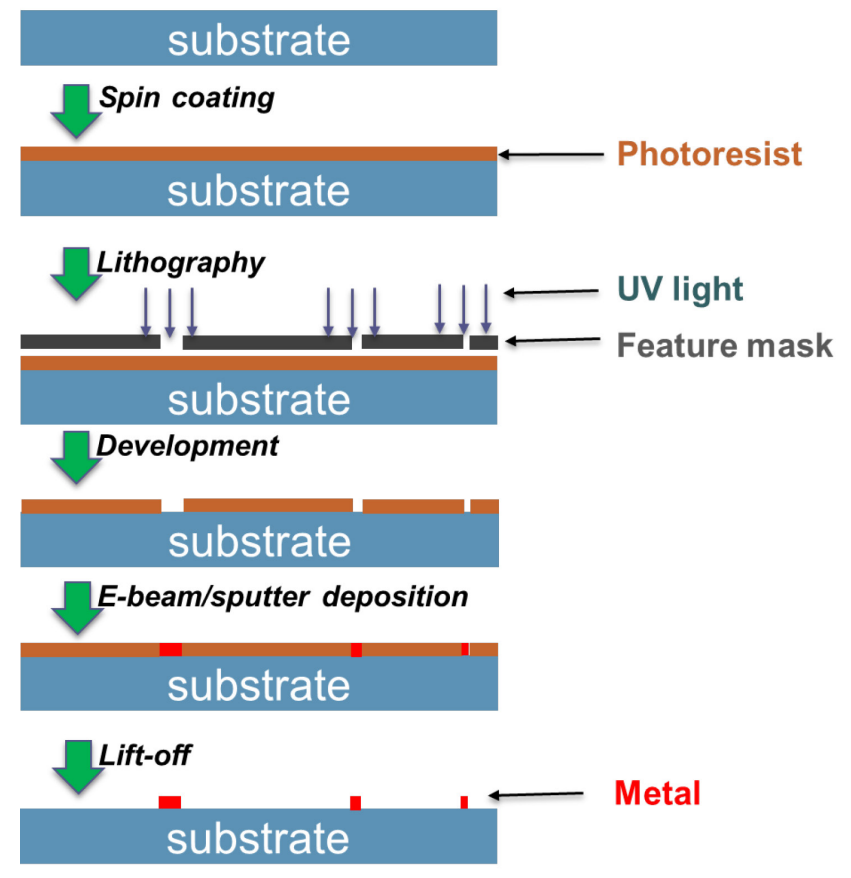

Figure AP 1 Demonstration of microfabrication processing flow 
It is important to choose the proper photoresist at the very beginning of the entire microfabrication operation. Photoresist is a UV light-sensitive polymer used in lithography to coat, then pattern features on a surface. Photoresists are engineered to be positive or negative. For positive photoresists, the polymer monomers exposed to light become soluble in the corresponding photoresist developer while the rest of portion remains insoluble; for negative type photoresists, the UV exposed portion becomes insoluble to photoresist developer while the rest of portion remains soluble and can be washed away. By choosing the right photoresist type, a feature that replicates or inversely replicates the photo mask can be obtained. The common positive and negative photoresists are S1800 series (Dow Electronic Materials) and NR9 series (Futurrex), respectively. The common developers for positive and negative photoresist are MIF series (Dow Electronic Materials) and RD series (Futurrex), respectively.

To obtain desirable feature resolutions using different photoresists, spin rate, light exposure energy, and hardbake time optimizations need to be carefully investigated. The spin rate data can be easily obtained from photoresist manufacture's website or manual calibration (shown in Figure AP 2). The exposure energy and hardbake times need to be optimized manually, although literature provides starting guidance. Table AP 1 demonstrates an example of one photoresist optimization matrix utilized in this study. As the example, photoresist NR9-1500P can achieve the best feature resolution at the conditions of $210 \mathrm{~J} / \mathrm{cm}^{2}$ UV intensity, $2000 \mathrm{rpm}$ spin rate and $1 \mathrm{~min}$ hardbake time. Figure AP 3 demonstrates the obtained pattern features before and after optimization. 


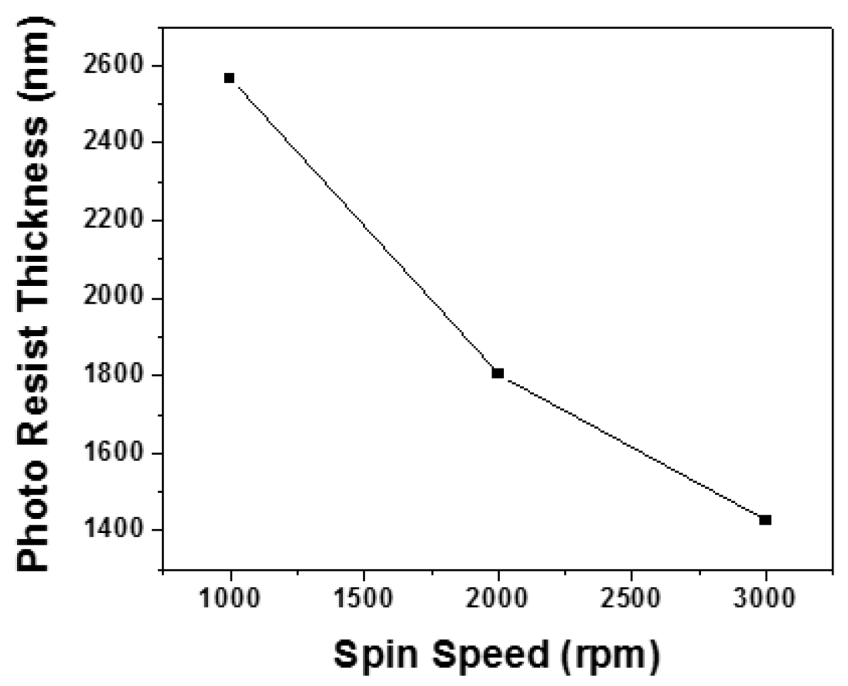

Figure AP 2 Spin speed-photoresist thickness curve measured via profilometer after hardbaking. Negative photoresist NR9$1500 p$ was utilized for this manual calibration.

Table AP 1 Example of NR9-1500p photoresist optimization matrix

\begin{tabular}{|c|c|c|c|}
\hline $\begin{array}{c}\text { Exposure } \\
\text { time }\end{array}$ & $\begin{array}{c}20 \mathrm{~s} \\
\left(211 \mathrm{~mJ} / \mathrm{cm}^{2}\right)\end{array}$ & $\begin{array}{c}22.5 \mathrm{~s} \\
\left(238 \mathrm{~mJ} / \mathrm{cm}^{2}\right)\end{array}$ & $\begin{array}{c}25 \mathrm{~s} \\
(265 \\
\left.\mathrm{mJ} / \mathrm{cm}^{2}\right)\end{array}$ \\
\hline $\begin{array}{c}\text { Spin } \\
\text { coating } \\
\text { speed }\end{array}$ & $\begin{array}{c}1000 \mathrm{rpm} \\
(2565-2835 \mathrm{~nm})\end{array}$ & $\begin{array}{c}2000 \mathrm{rpm} \\
(1805-1995 \mathrm{~nm})\end{array}$ & $\begin{array}{c}3000 \mathrm{rpm} \\
(1425-1575 \\
\mathrm{nm})\end{array}$ \\
\hline $\begin{array}{c}\text { Hardbake } \\
\text { time }\end{array}$ & $1 \mathrm{~min}$ & $2 \mathrm{~min}$ & $3 \mathrm{~min}$ \\
\hline
\end{tabular}



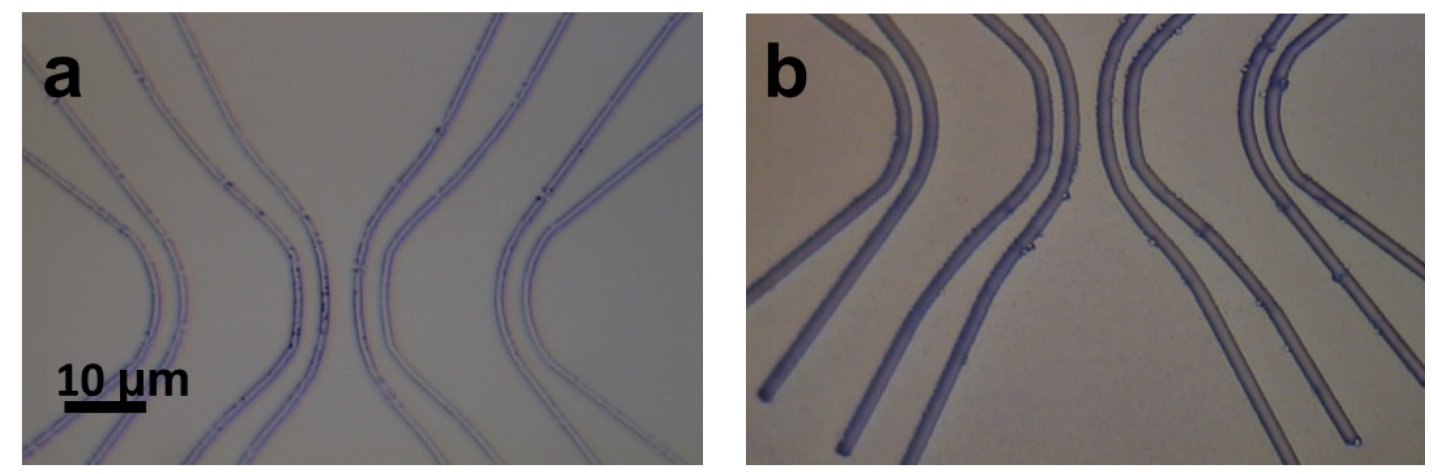

Figure AP 3 Demonstration of NR9-1500P photoresist optimization of lithography process. a) microscope images of patterns created using microfabrication lab default parameters: $200 \mathrm{~J} / \mathrm{cm}^{2} \mathrm{UV}$ intensity, $1000 \mathrm{rpm}$ spin rate, and 1 min hardbake. b) microscope images of patterns created at optimized conditions: $210 \mathrm{~J} / \mathrm{cm}^{2}$ UV intensity, $2000 \mathrm{rpm}$ spin rate, and 1 min hardbake.

All the microfabrication steps are completed in Class 1000 softwall cleanroom in the Microfabrication Core Facility (MFF) located on the $4^{\text {th }}$ floor of the M\&M Building, Michigan Technological University. The instruments employed to manufacture sIEF chips for this dissertation included clean room, lithography mask aligner (EVG 620), plasma cleaner (Trion Technology Phantom II), electron beam deposition (E-beam, Denton DV-502A), sputter deposition (Perkin Elmer 2400 Sputter Tool - 6 inch and 8 inch), profilometer (Perthometer S2, Mahr), and ellipsometer (J.A. Woollam VASE). Each instrument requires training and check-out with the MFF lab director before individual operation. To check the instrument details, schedule, training details and chemical supplies, please check the Microfabrication Core Facility website: https://www.mtu.edu/microfabrication.

Suggested Standard Operating Procedures (SOP) for the main microfabrication instruments are listed as follows (additional optimization will be required if using different process):

\section{A. Photolithography Masking and Feature Development}

Electrode pair features on microscope glass slide are completed by photolithography under homemade film mask: 
a. Clean slide surface. Sonication 5 min with Acetone (CMOS grade)/IPA (CMOS grade)/DI water, air dry it and further dry on a hot plate $\left(120^{\circ} \mathrm{C}\right)$ for 2 min.

b. Spin photoresist Futurrex PR1-1000A following the steps below to get a $0.7 \mu \mathrm{m}-2.1 \mu \mathrm{m}$ layer.

- Spin at 800rpm for $15 \mathrm{sec}$ with acceleration=100

- Spin at 800rpm for $30 \mathrm{sec}$ with acceleration $=300$

c. Prebake on hotplate $120^{\circ} \mathrm{C}, 30 \mathrm{~s}$

d. Soft contact. Expose $210 \mathrm{~J} / \mathrm{cm}^{2}$. Antireflective coating required on the backside - use blue dicing saw tape for this purpose. Operations are as followed:

- Setup EVG machine

- Check EVG machine for green light

- Turn on EVG machine at key switch and computer CPU

- Open EVG software

- Install appropriate lithography mask (follow along with computer instructions)

Important parameters:

- Mask thickness: no change

- Mask size: 5 inch

- Substrate size: 4 inch

- Substrate thickness: $1 \mathrm{~mm}$

- Process time: exposure time is equal to the incident energy (210 $\mathrm{J} / \mathrm{cm}^{2}$ ) divided by incident light intensity (in $W / \mathrm{cm}^{2}$ ), which can be measured at the beginning of lithography process.

e. Post-bake on hotplate $120^{\circ} \mathrm{C}$ for $30 \mathrm{~s}$

f. Develop with undiluted photoresist Futurrex RD6, $15 \mathrm{~s}$ (minimal agitation)

g. Immerse in DI water to stop reaction, and further rinse with plenty of DI water

To obtain the best feature resolution, operation conditions should be optimized based on variables including exposure energy, spin-coating speed (photoresist thickness) and photoresist baking time. An example optimization matrix is listed in the following table. For a new photoresist employed, the spin speed-thickness calibration curve can be found in manufactures website as reference, however, the optimization matrix should be still reapplied due to the different experiment conditions. 


\section{B. RIE/ICP Etch System for Slide Pre-Deposit Cleaning}

\section{a. Sample loading steps are as following}

- Select cancel from touchscreen (remember to always be wearing gloves when touching screen)

- Select "vent chamber"

- Wait until chamber has vented and the lid opens

- Load sample with O-rings surrounding sample

- Select "chamber close" from touchscreen menu

\section{b. Cleaning steps are as following}

- Select PR-1 from the files menu

- Turn on oxygen at tank

Input suitable process parameters from "manual process control" menu

- RIE: 500

- ICP: 50

- O2 flow: 100

- Time: $15 \mathrm{sec}$

- Pressure: 150

c. Once system has pumped down completely run recipe by going to "automatic process control". After process has ended put system in standby mode for 5 minutes and then elect "vent chamber", remove sample and select "chamber close" and allow system to pump down completely

\section{Electrode Metal Layer Deposition and Liftoff}

Electrodes metal layer can be obtained by E-beam (a) or sputter deposition (b). E-beam deposition rate can be simultaneously read from the display window; sputter deposition rate can be read from the pre-calibrated data sheet in Microfabrication Core Facilities, however, the calibration is suggested to be redone every year to ensure the accuracy. The recalibration curve can be achieved by depositing metal in standard silicon wafer at different deposition length and recording the corresponding deposition thickness using ellipsometer. 


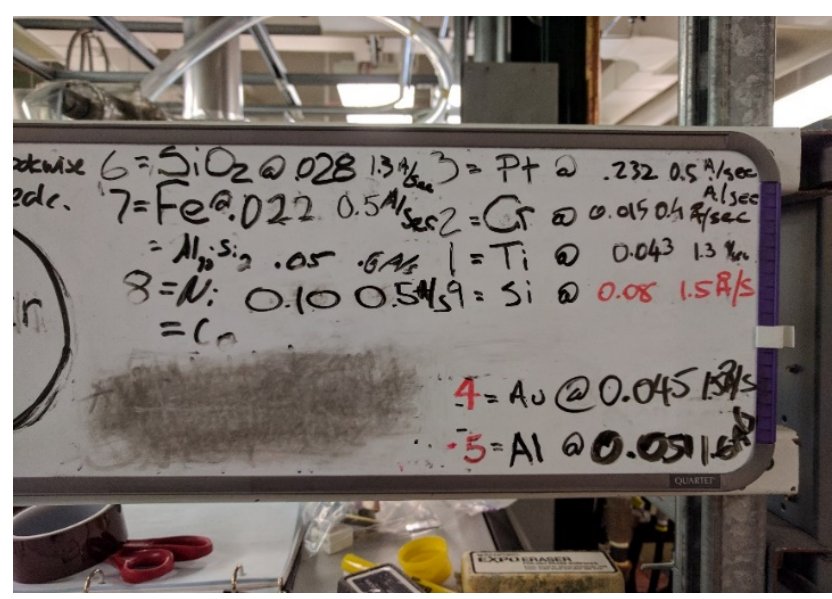

Figure AP 4 E-beam information board in Microfabrication Core Facilities. Metal or oxides crucibles ( $\mathrm{Ti}, \mathrm{Cr}, \mathrm{Pt}, \mathrm{Au}, \mathrm{Al}$, $\mathrm{SiO}_{2}, \mathrm{Fe}, \mathrm{Ni}$ ) are numbered from 1 to 8 . The real time deposition rate are wrote down near each metal names.
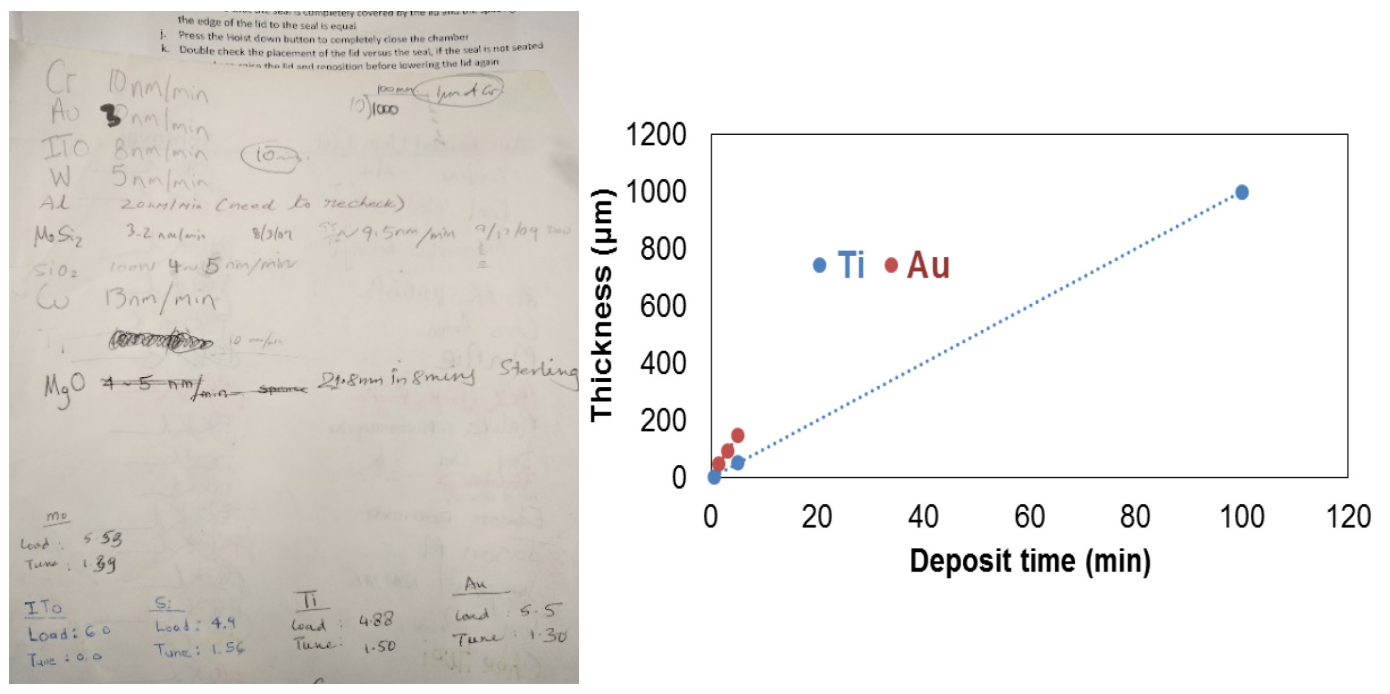

Figure AP 5 Suggested sputter deposition rate calibration data sheet offered by Microfabrication Core Facilities (left) and a demonstrated manual calibration curve of $\mathrm{Au}$ and $\mathrm{Ti}$ (right). If a process requires accurate metal deposition thickness information, a manual calibration is suggested before starting the process. 


\section{a. E-beam Ti-Au Deposition}

Pump up from high vacuum to atmospheric pressure

- Turn off high vacuum gauge off

- Turn knob to Manual Pump

- Turn on Mechanical pump

- Let Mechanical pump warm up

- Turn vent valve on

- Pull lever to open chamber door, letting vent continue until door opens easily

- Check the temperature near bottom of Ebeam, should read $15 \mathrm{~K}$ (if not inform lab manager)

- Once door easily opens, unscrew circle plate and place the substrates on it

- Change glass slides on chamber door

- Change the crucible position to correct metal

- Check the O-ring (door seal)

- Close door but do not lock it

- Turn vent valve off

- Lock chamber door

Pump down to high vacuum

- Turn knob to Auto Pump

- Wait until red light for high vacuum to come on (this will occur when pressure reads $\sim 120$ mTorr, transition point)

- Turn on high vacuum gauge

- Turn off mechanical pump

- Vent mechanical pump line

- Pump Down for 3+ hrs

$1^{\text {st }}$ layer deposition - Ti

- Turn on all power on (supply/monitor/water/controller/rotation)

- Wait for 5 min

- Change the program setting to Ti (its number input, check whiteboard to get the number for $\mathrm{Ti}$ )

- Turn ON current power and slowly turn UP the current

- Turn up current power half way to center beam (halfway of starting point listed on whiteboard)

- Wait till Ti melt (about $5 \mathrm{~min}$ ), beam should be positioned at the center of crucible (stable deposition rate $1 \mathrm{k} \AA / \mathrm{min}$ recommended)

- Open shutter and deposit Ti $\sim 0.500 \mathrm{kA}$ (500nm) 
- After reaching target thickness, close shutter

- Turn down all current power all the way down (knob should be turned all the way to the left) and turn off current power

- Let system cool for 5 min $2^{\text {nd }}$ layer deposition $(\mathrm{Au})$

- Change the crucible to Au position (using whiteboard)

- Change the program to Au (number input)

- Turn ON current power and turn UP current halfway

- Center beam on Au crucible. Once Au molten (about $1 \mathrm{~min}$ ) do not wait longer than a minute (saves on Au material)

- Do not expose Au to high currents (<2 A. Large value can evaporate all of gold and burn a hole through crucible)

- Open shutter and deposit Au $0.500 \mathrm{kA}$ (500nm)

- Close shutter

- Turn down current

- Turn off current

- Turn off controller

- Turn off power/water/monitor/rotation

- Wait 5 min before turning off main power switch (bottom flip switch) Pump up from high vacuum to atmospheric pressure

- Turn off high vacuum gauge off

- Turn knob to Manual Pump

- Turn on Mechanical pump

- Let Mechanical pump warm up

- Turn vent valve on

- Pull lever to open the door, letting vent continue until door opens easily

- Check the temperature near bottom of E-beam, $15 \mathrm{~K}$ (if not inform lab manager)

- Once door easily opens, unscrew circle plate and remove substrates

- Check the O-ring (door seal)

- Close door but do not lock it

- Turn Vent valve off

- Lock the door

- Pump down to high vacuum

- Turn knob to Auto Pump

- Wait until red light for high vacuum is on, pressure will be around $\sim 120$ mTorr which is a transition point 
- Turn on the high vacuum gauge

- Turn off mechanical pump

- Vent mechanical pump line

b. Sputter Deposition: chamber is pre-vacuumed at $5 \times 10^{-7}$ Torr. Gas flow for plasma generation are 7 7.5 sccm Ar flow and $0.5 \mathrm{sccm} \mathrm{O}_{2}$ flow, respectively. Operation pressure is $7.5 \times 10^{-3}$ Torr. Plasma power is fixed at $100 \mathrm{~W}$. Ti deposition rate is $10 \mathrm{~nm} / \mathrm{s}$, Au deposition rate is $30 \mathrm{~nm} / \mathrm{s}$. The final $\mathrm{Ti}$ and $\mathrm{Au}$ layer thickness are $5 \mathrm{~nm}$ and $95 \mathrm{~nm}$, respectively. The detailed SOP are as following:

Pump up to atmosphere from high vacuum

- Turn ion gauge off

- Close the high vac valve

- Open vent valve

- Wait for the chamber to reach atmospheric pressure

- Open the chamber

- Pump down from atmosphere to $5 \times 10^{-2}$ Torr

- Put substrate in the chamber as close to the center of the target as possible

- Close the chamber almost all the way

- Close the vent valve

- Close the chamber completely, making sure it lines up to avoid leaks

- Close the foreline valve

- Open the roughing valve (the foreline and roughing valves should never be open at the same time even though the system does not have a failsafe to prevent this)

- Wait for the pressure to reach at least $5 \times 10^{-2}$ Torr

- Pump from $50 \mathrm{mTorr}$ to high vacuum

- Close the roughing valve

- Open the foreline valve

- Wait for the foreline to evacuate (the pressure reading will stabilize)

- Open the hi-vac valve

- Turn on the ion gauge when the chamber is at $10^{-3}$

- Wait for the chamber to reach the desired pressure of $2 \sim 3 \times 10^{-6}$ (This can take 2.5-3 hours so you can leave when the pressure is steadily dropping- usually less than $10^{-3}$ )

- $\quad$ Sputtering 
- Turn off the ion gauge

- Turn on the water for head

- Turn on the power supply

- Close throttle valve

- Turn on the gas (at the tank and the valve)

- Set gas flow to $10 \mathrm{sccm}$

- Turn on tuning control (this light should normally come on but may not because of a blown fuse)

- Change process to sputter deposit

Titanium Sputtering

- Need to pre-clean the titanium target by moving the Table to Target 1 (Farthest from Target 3)

- Set the tuning cap positions

- Turn up the power until the plasma lights (reflected power to a little above 10 and press ignition switch).

- Reduce the reflected power $(=0)$ and increase forward power $(=10)$

- Adjust the tuning cap positions as needed to achieve the power settings

- Let run for 5 minutes

- Move the table back to Target 3

- Set the tuning cap positions

- Turn up the power until the plasma lights (reflected power to a little above 10 and press ignition switch).

- Reduce the reflected power $(=0)$ and increase forward power $(=10)$

- Adjust the tuning cap positions as needed to achieve the power settings

- Let run for 5 minutes

\section{Gold Sputtering}

- No pre-cleaning required

- Move the table to Target 2

- $\quad$ Set the Target to Target 2

- Set the tuning cap positions

- Turn up the power until the plasma lights (reflected power to a little above 10 and press ignition switch).

- Reduce the reflected power $(=0)$ and increase forward power $(=10)$ 
- Adjust the tuning cap positions as needed to achieve the power settings

- Let run for desired length of time (deposits at a rate of $30 \mathrm{~nm} / \mathrm{min}$ ) if longer than 5 minutes do 5 minutes on and 2 minute cool down in between each 5 minute period

Unload sample

- Set the table position to "etch"

- Turn down the argon flow and switch the valve off (on equipment and at tank)

- Turn off power

- Venting the chamber

- Make sure the gas is turned off and then open the throttle valve (put in down position)

- Close the hi-vac valve

- Open the vent valve

- Wait for the chamber to reach atmospheric pressure

- Shut vent valve

- Open the chamber and remove samples

- Pump down to hi-vac when finished

Liftoff photoresist with undiluted Futurrex RR4 or Acetone until excess metal is removed (sonication $5 \mathrm{~min}$ or stay still $30 \mathrm{~min}$ )

\section{Passivation Layer: $50 \mathrm{~nm}$ of Sputter Deposited $\mathrm{HfO}_{2}$ Film with 8" Hafnium Target.}

a. $\mathrm{HfO}_{2}$ passivation layer is obtained via sputter deposition: at $3 \times 10^{-7}$ Torr:

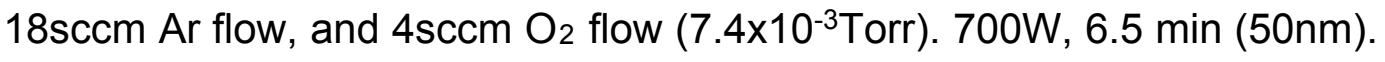
Detailed operations are the same to Ti/Au deposition. Contact pads area is protected by thermal tape.

b. $\mathrm{HfO}_{2}$ anneal on hot plate: $250^{\circ} \mathrm{C}, 30 \mathrm{~min}$ (ramp temperature up from room temperature, and let cool down slowly, do not move it off the hot plate if temperature is higher than $100 \mathrm{C}$ ). Also place glass wafer on top of a $>4$ " $\mathrm{Si}$ wafer to increase heating uniformity.

\section{Conclusions:}

Achieving highly reproducible microdevices in the most efficient time-frame possible can be accomplished by a) verifying the actual parameters (i.e. film thickness, quality, device yield rate, etc.) as you go in the process and b) tabulating those results every time they are measured in a comparison table. Microfabrication is partially an art, but optimizing the skill requires documentation of conditions 
utilized making it more controllable with good scientific documentation skills. In summary, the items to track regularly include:

- Film thickness of photoresist/dielectric films, measurable via elipsometry (thickness $>1 \mu \mathrm{m}$, opaque substrates) or profilometry (thickness $>1 \mu \mathrm{m}$, transparent substrates).

- $\quad$ Real time UV light beam intensity (perform intensity test in EVG machine) and exposure energy.

- Hardbake time and develop time (may vary according to temperature and humidity. Keep record of real time temperature and humidity is suggested)

- $\quad$ Lift-off chemical and process time.

- $\quad$ Lithography pattern quality check using microscope, record at least one microscope images.

- If plasma clean or etch is performed, record power, process time, gas flow rate, and plasma color.

- For metal/dielectric layer deposition, record process time, gas flow rate, chamber vacuum level, processing vacuum level, and final deposition thickness (need to be measured by elipsometry or profilometry). Check crucial status before use. 
A suggested check-out list can be seen in the table below, it should be modified and updated based on real process operations.

Table AP 2 Microfabrication process parameters check out sheet

\begin{tabular}{|c|c|c|c|c|c|}
\hline Process & User & \multicolumn{4}{|c|}{ Key parameters } \\
\hline $\begin{array}{l}\text { Spin } \\
\text { coating }\end{array}$ & & $\begin{array}{c}\text { Photoresist } \\
\text { used }\end{array}$ & $\begin{array}{c}\text { Spin } \\
\text { speed/recipe } \\
\text { and real film } \\
\text { thickness }\end{array}$ & $\begin{array}{l}\text { Prebake } \\
\text { and } \\
\text { hardbake } \\
\text { time }\end{array}$ & $\begin{array}{l}\text { Temperature } \\
\text { and humidity }\end{array}$ \\
\hline Lithography & & $\begin{array}{l}\text { Lamp } \\
\text { intensity } \\
\text { and } \\
\text { process } \\
\text { energy }\end{array}$ & $\begin{array}{c}\text { Substrate } \\
\text { type and } \\
\text { transparency }\end{array}$ & $\begin{array}{l}\text { Photoresist } \\
\text { developer } \\
\text { used }\end{array}$ & $\begin{array}{l}\text { Develop } \\
\text { time }\end{array}$ \\
\hline $\begin{array}{c}\text { Plasma } \\
\text { clean/etch }\end{array}$ & & $\begin{array}{l}\text { Process } \\
\text { energy }\end{array}$ & $\begin{array}{l}\text { Gas flow } \\
\text { rate }\end{array}$ & $\begin{array}{c}\text { Plasma } \\
\text { color }\end{array}$ & $\begin{array}{c}\text { Process } \\
\text { time }\end{array}$ \\
\hline $\begin{array}{l}\text { Thin layer } \\
\text { deposition }\end{array}$ & & $\begin{array}{l}\text { Process } \\
\text { energy }\end{array}$ & $\begin{array}{l}\text { Deposition } \\
\text { time (record } \\
\text { room } \\
\text { temperature } \\
\text { and } \\
\text { humidity) }\end{array}$ & $\begin{array}{l}\text { Vacuum } \\
\text { level } \\
\text { (during } \\
\text { pumping } \\
\text { down and } \\
\text { processing) }\end{array}$ & $\begin{array}{l}\text { Real film } \\
\text { thickness }\end{array}$ \\
\hline
\end{tabular}




\section{Appendix B: Permission Letters of Reprinted Figures}

\section{Permission of Figure 1.3}

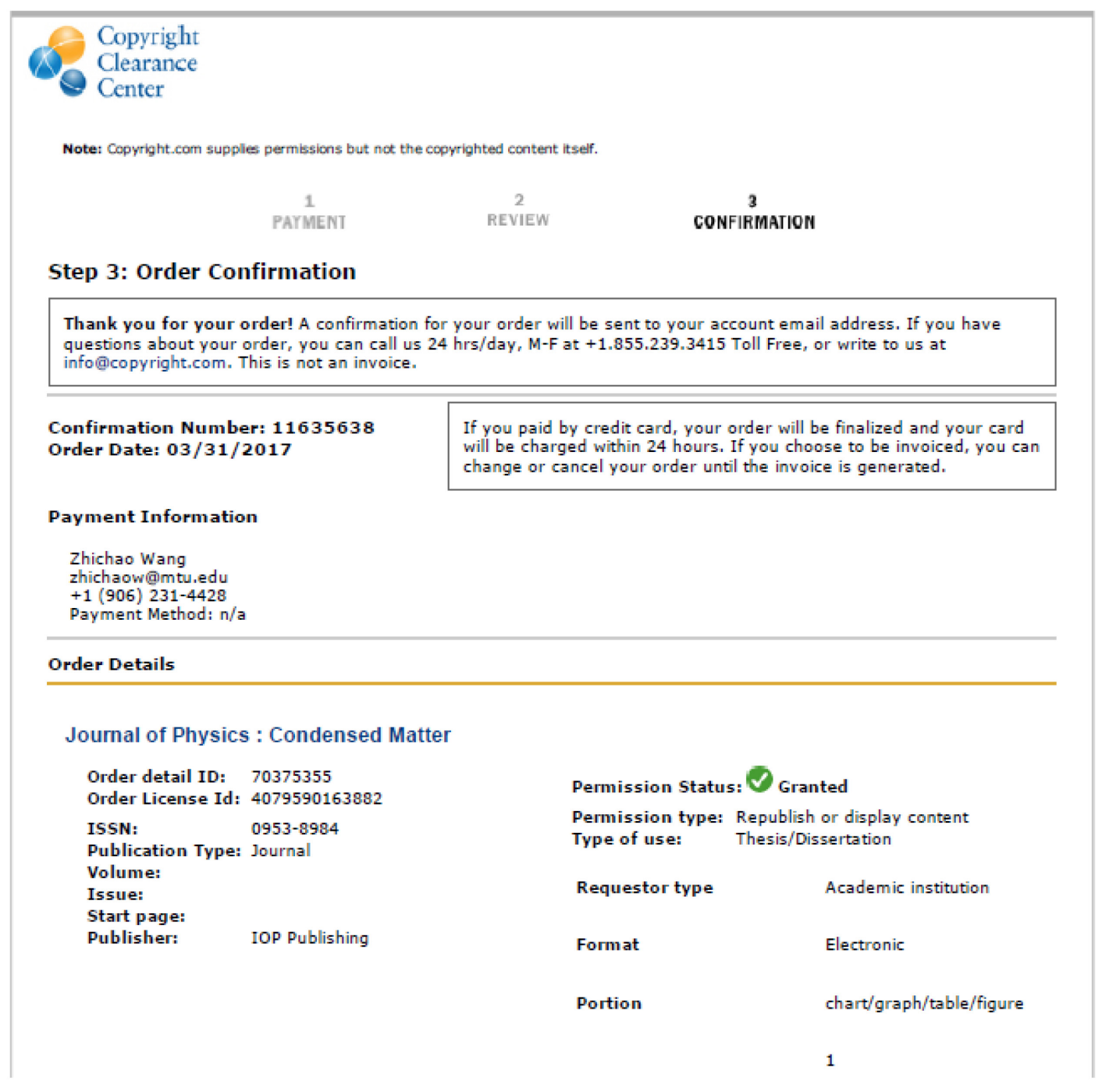


Number of

charts/graphs/tables/f

igures

Title or numeric

reference of the

Figure 25

portion( $s$ )

Title of the article or

chapter the portion is

from

7.1. Lab-on-a-chip

Editor of portion(s)

N/A

Author of portion(s)

N/A

Volume of serial or

monograph

N/A

Page range of portion

R735

Publication date of

portion

2005

Rights for

Main product

Duration of use

Life of current edition

Creation of copies for

the disabled

no

With minor editing

privileges

no

For distribution to

United States

In the following

language(s)

Original language of publication

With incidental

promotional use

no 
Lifetime unit quantity

of new product

Made available in the following markets

The requesting

person/organization

Order reference number

Author/Editor

The standard identifier of New Work

The proposed price

Title of New Work

Publisher of New Work

Expected publication

date

Estimated size (pages)
Up to 499

education

Zhichao Wang

Zhichao Wang

Dissertation/thesis

SURFACE ENABLED LABON-A-CHIP (LOC) DEVICE

FOR PROTEIN DETECTON AND SEPARATION

Michigan Technological

University

May 2017

160 


\section{Permission of Figure 2.2}

$330 / 2017$

RightsLink Printable Lucense

AIP PUBLISHING LLC LICENSE TERMS AND CONDITIONS

Mar 30,2017

This Agreement between Zhichao Wang ("You") and AIP Publishing LIC ("AIP Publishing LLC') consists of your license details and the terms and conditions provided by AIP Publishing LLC and Copyright Clearance Center.

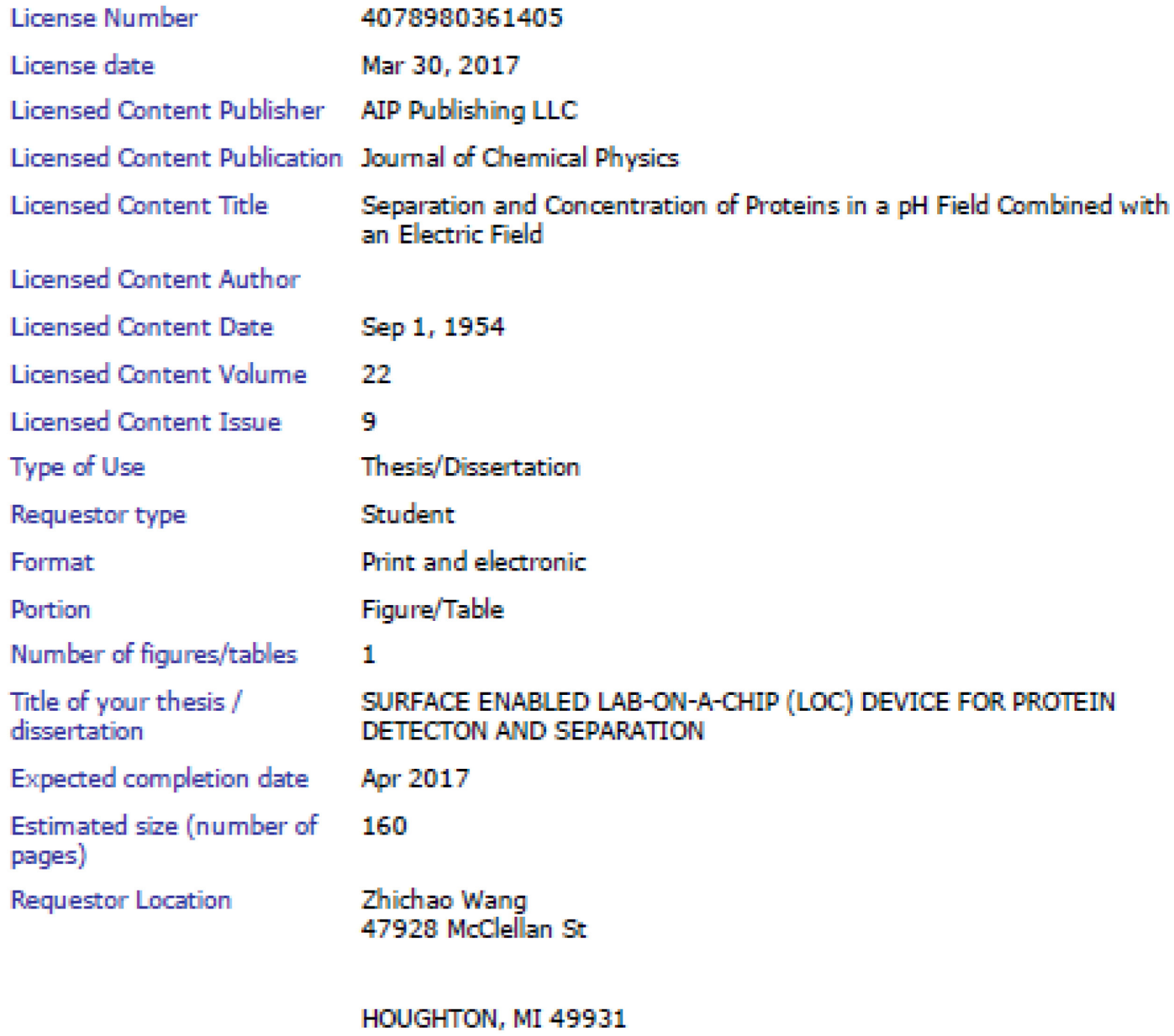

HOUGHTON, MI 49931 


\section{Permission of Figure 2.3}

ELSEVIER LICENSE

TERMS AND CONDITIONS

Mar 31, 2017

This Agreement between Zhichao Wang ("You") and Elsevier ("Elsevier") consists of your license details and the terms and conditions provided by Elsevier and Copyright Clearance Center.

\begin{tabular}{|c|c|}
\hline License Number & 4079580719924 \\
\hline License date & Mar 31,2017 \\
\hline Licensed Content Publisher & Elsevier \\
\hline Licensed Content Publication & Biochemical and Biophysical Research Communications \\
\hline Licensed Content Title & $\begin{array}{l}\text { Polyacrylamide-isoelectric-focusing a new technique for the } \\
\text { electrophoresis of proteins }\end{array}$ \\
\hline Licensed Content Author & D.H. Leaback, A.C. Rutter \\
\hline Licensed Content Date & 13 August 1968 \\
\hline Licensed Content Volume & 32 \\
\hline Licensed Content Issue & 3 \\
\hline Licensed Content Pages & 7 \\
\hline Start Page & 447 \\
\hline End Page & 453 \\
\hline Type of Use & reuse in a thesis/dissertation \\
\hline $\begin{array}{l}\text { Intended publisher of new } \\
\text { work }\end{array}$ & other \\
\hline Portion & figures/tables/-lustrations \\
\hline $\begin{array}{l}\text { Number of } \\
\text { figures/tables/illustrations }\end{array}$ & 1 \\
\hline Format & both print and electronic \\
\hline $\begin{array}{l}\text { Are you the author of this } \\
\text { Elsevier article? }\end{array}$ & No \\
\hline Will you be translating? & No \\
\hline \multicolumn{2}{|l|}{ Order reference number } \\
\hline Original figure numbers & figure 1 \\
\hline
\end{tabular}


Title of your

thesis/dissertation

Expected completion date

Estimated size (number of pages)

Elsevier VAT number

Requestor Location

$\begin{array}{ll} & \begin{array}{l}\text { HOUGHTON, MI } 49931 \\ \text { United States } \\ \text { Attn: Zhichao Wang }\end{array} \\ \text { Publisher Tax ID } & 98-0397604 \\ \text { Total } & 0.00 \text { USD }\end{array}$

Terms and Conditions

Apr 2017

160

GB 494627212

Zhichao Wang

47928 McClellan St

HOUGHTON, MI 49931

United States

tn: Zhichao Wang

0.00 USD
SURFACE ENABLED LAB-ON-A-CHIP (LOC) DEVICE FOR PROTEIN

DETECTON AND SEPARATION

\section{INTRODUCTION}

1. The publisher for this copyrighted material is Elsevier. By clicking "accept" in connection with completing this licensing transaction, you agree that the following terms and conditions apply to this transaction (along with the Billing and Payment terms and conditions established by Copyright Clearance Center, Inc. ("CCC"), at the time that you opened your Rightslink account and that are available at any time at hitb://mvaccount.copvrightcom).

\section{GENERAL TERMS}

2. Elsevier hereby grants you permission to reproduce the aforementioned material subject to the terms and conditions indicated.

3. Acknowledgement: If any part of the material to be used (for example, figures) has appeared in our publication with credit or acknowledgement to another source, permission must also be sought from that source. If such permission is not obtained then that material may not be included in your publication/copies. Suitable acknowledgement to the source must be made, either as a footnote or in a reference list at the end of your publication, as follows:

"Reprinted from Publication title, Vol /edition number, Author(s), Title of article / title of chapter, Pages No., Copyright (Year), with permission from Elsevier [OR APPLICABLE SOCIETY COPYRIGHT OWNER]." Also Lancet special credit - "Reprinted from The Lancet, Vol. number, Author(s), Title of article, Pages No., Copyright (Year), with permission from Elsevier."

4. Reproduction of this material is confined to the purpose and/or media for which permission is hereby given. 


\section{Permission of Figure 2.4}

\section{ROYAL SOCIETY OF CHEMISTRY LICENSE TERMS AND CONDITIONS}

This Agreement between Zhichao Wang ("You") and Royal Society of Chemistry ("Royal Society of Chemistry") consists of your license details and the terms and conditions provided by Royal Society of Chemistry and Copyright Clearance Center.

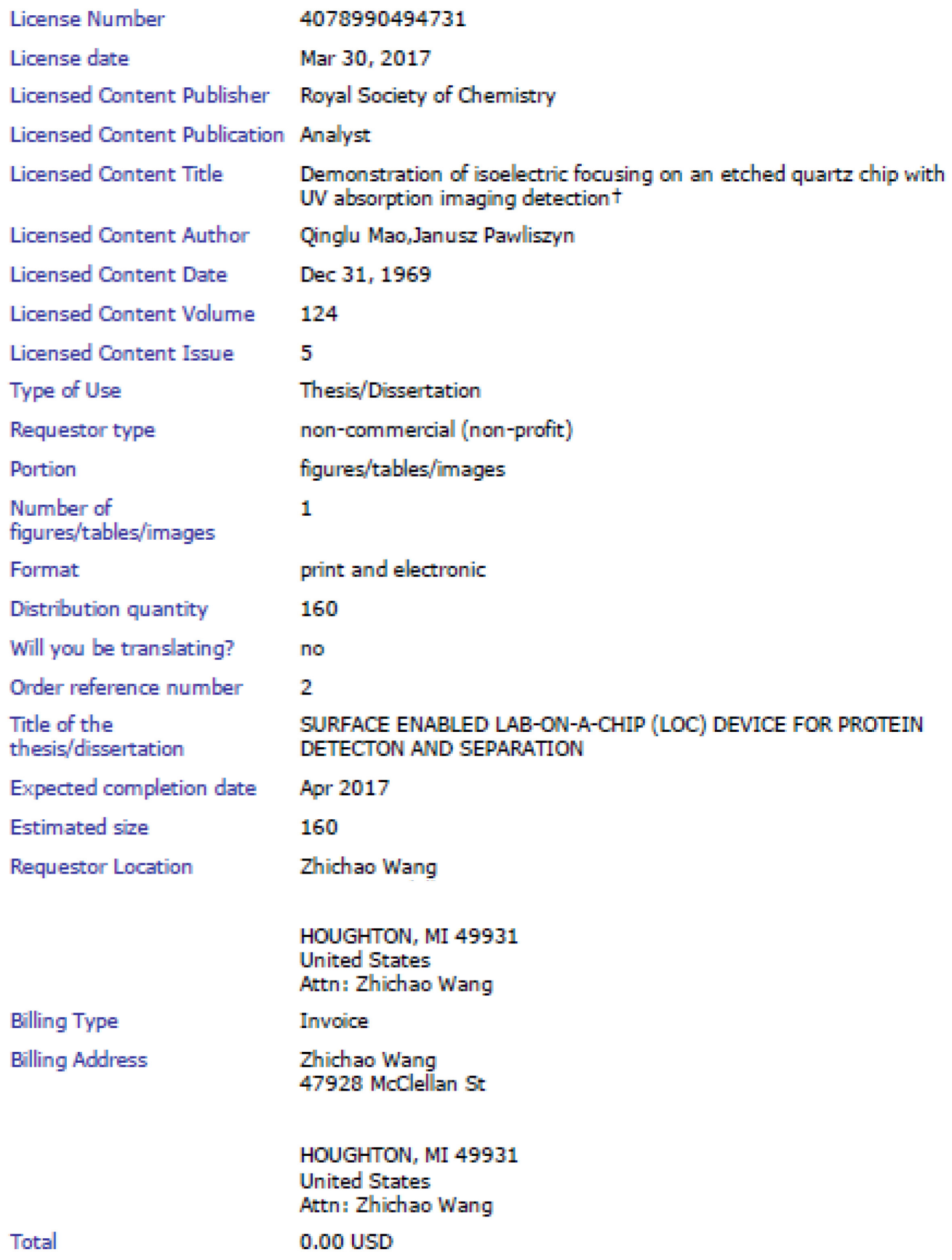




\section{Permission of Figure 2.5}

23 Copyright

Clearan

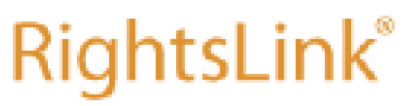

ACSPublications: Title:

Mast Truitud. Mest Cted. Mast Boud.
Isoelectric Focusing in a

Poly(dimethylsilocane)

Microfluidic Chip

Author:

Huanchun Cui, Keisuke Horiuchi,

Prashanta Dutta, et al

Publication: Analytical Chemistry

Publisher: American Chemical Society

Date: $\quad$ Mar 1, 2005

Copyright 12005 , American Chemical Society

Logged in as:

Zhichas Wang

Acoount:

3001132995

LogauT

\section{PERMISSION/LICENSE IS GRANTED FOR YOUR ORDER AT NO CHARGE}

This type of permission/license, instead of the standard Terms \& Conditions, is sent to you because no fee is being charged for your order. Please note the following:

- Permission is granted for your request in both print and electronic formats, and translations.

- If figures and/or tables were requested, they may be adapted or used in part.

- Please print this page for your records and send a copy of it to your publisher/graduate school.

- Appropriate credit for the requested material should be given as follows: "Reprinted (adapted) with permission from (COMPLETE REFERENCE CITATION). Copyright (YEAR) American Chemical Society." Insert appropriate information in place of the capitalized words.

- One-time permission is granted only for the use specified in your request. No additional uses are granted (such as derivative works or other editions). For any other uses, please submit a new request.

If credit is given to another source for the material you requested, permission must be obtained from that source. 


\section{References:}

1. Mugele, F. and J.-C. Baret, Electrowetting: from basics to applications. Journal of Physics: Condensed Matter, 2005. 17(28): p. R705.

2. Kolin, A., Separation and Concentration of Proteins in a $\mathrm{pH}$ Field Combined with an Electric Field. The Journal of Chemical Physics, 1954. 22(9): p. 1628-1629.

3. Mao, Q. and J. Pawliszyn, Demonstration of isoelectric focusing on an etched quartz chip with UV absorption imaging detection[dagger]. Analyst, 1999. 124(5): p. 637-641.

4. Cui, H., et al., Isoelectric Focusing in a Poly(dimethylsiloxane) Microfluidic Chip. Analytical Chemistry, 2005. 77(5): p. 1303-1309.

5. Leaback, D., Polyacrylamide-isoelectric-focusing a new technique for the electrophoresis of proteins. Biochemical and biophysical research communications, 1968. 32(3): p. 447-453.

6. Jesus-Perez, N.M. and B.H. Lapizco-Encinas, Dielectrophoretic monitoring of microorganisms in environmental applications. Electrophoresis, 2011. 32(17): p. 2331-2357.

7. McDonald, J.C., et al., Fabrication of microfluidic systems in poly(dimethylsiloxane). Electrophoresis, 2000. 21(1): p. 27-40.

8. Duffy, D.C., et al., Rapid prototyping of microfluidic systems in poly(dimethylsiloxane). Analytical Chemistry, 1998. 70(23): p. 4974-4984.

9. Anwar, K., T. Han, and S.M. Kim, Reversible sealing techniques for microdevice applications. Sensors and Actuators B-Chemical, 2011. 153(2): p. 301-311.

10. Wilding, P., M.A. Shoffner, and L.J. Kricka, PCR IN A SILICON MICROSTRUCTURE. Clinical Chemistry, 1994. 40(9): p. 1815-1818.

11. Vindeløv, L.L. and I.J. Christensen, A review of techniques and results obtained in one laboratory by an integrated system of methods designed for routine clinical flow cytometric DNA analysis. Cytometry, 1990. 11(7): p. 753-770.

12. Horsman, K.M., et al., Forensic DNA Analysis on Microfluidic Devices: A Review. Journal of Forensic Sciences, 2007. 52(4): p. 784-799.

13. Rosenberg, I.M., Protein analysis and purification: benchtop techniques. 2013: Springer Science \& Business Media.

14. Branden, C.I., Introduction to protein structure. 1999: Garland Science.

15. Lodish, H.F., Molecular Cell Biology. 2000: W. H. Freeman.

16. Berg JM, Tymoczko JL, and S. L., DNA Illustrates the Relation between Form and Function, in Biochemistry. 2002, W. H. Freeman: New York.

17. Domon, B. and R. Aebersold, Mass Spectrometry and Protein Analysis. Science, 2006. 312(5771): p. 212-217.

18. Hames, B.D., Gel Electrophoresis of Proteins: A Practical Approach. 1998: OUP Oxford.

19. Bury, A.F., Analysis of protein and peptide mixtures. Journal of Chromatography A, 1981. 213(3): p. 491-500.

20. O'Farrell, P.H., High resolution two-dimensional electrophoresis of proteins. Journal of Biological Chemistry, 1975. 250(10): p. 4007-4021.

21. Righetti, P.G., et al., Carrier ampholytes for IEF, on their fortieth anniversary (1967-2007), brought to trial in court: The verdict. ELECTROPHORESIS, 2007. 28(21): p. 3799-3810. 
22. Görg, A., W. Weiss, and M.J. Dunn, Current two-dimensional electrophoresis technology for proteomics. PROTEOMICS, 2004. 4(12): p. 3665-3685.

23. Mahmood, T. and P.-C. Yang, Western Blot: Technique, Theory, and Trouble Shooting. North American Journal of Medical Sciences, 2012. 4(9): p. 429-434.

24. Karas, M. and F. Hillenkamp, Laser desorption ionization of proteins with molecular masses exceeding 10,000 daltons. Analytical Chemistry, 1988. 60(20): p. 22992301.

25. Medzihradszky, K.F., et al., The Characteristics of Peptide Collision-Induced Dissociation Using a High-Performance MALDI-TOF/TOF Tandem Mass Spectrometer. Analytical Chemistry, 2000. 72(3): p. 552-558.

26. Zhang, J., C. Das, and Z.H. Fan, Dynamic coating for protein separation in cyclic olefin copolymer microfluidic devices. Microfluidics and Nanofluidics, 2007. 5(3): p. 327-335.

27. Lengyel, T. and A. Guttman, Effect of linear polymer additives on the electroosmotic characteristics of agarose gels in ultrathin-layer electrophoresis. Journal of Chromatography A, 1999. 853(1-2): p. 511-518.

28. Minerick, A.R., The rapidly growing field of micro and nanotechnology to measure living cells. Aiche Journal, 2008. 54(9): p. 2230-2237.

29. Terry, S.C., J.H. Jerman, and J.B. Angell, GAS-CHROMATOGRAPHIC AIR ANALYZER FABRICATED ON A SILICON-WAFER. leee Transactions on Electron Devices, 1979. 26(12): p. 1880-1886.

30. Pohl, H.A. and J.S. Crane, Dielectrophoresis of Cells. Biophysical Journal, 1971. 11(9): p. 711-727.

31. Gascoyne, P.R.C., et al., Dielectrophoretic Separation of Mammalian-Cells Studied by Computerized Image-Analysis. Measurement Science \& Technology, 1992. 3(5): p. 439-445.

32. Walters, M.C. and H.T. Abelson, Interpretation of the complete blood count. Pediatric clinics of North America, 1996. 43(3): p. 599-622.

33. Santini, J.T., M.J. Cima, and R. Langer, A controlled-release microchip. Nature, 1999. 397(6717): p. 335-338.

34. Zhang, B., F. Foret, and B.L. Karger, A microdevice with integrated liquid junction for facile peptide and protein analysis by capillary electrophoresis/electrospray mass spectrometry. Analytical chemistry, 2000. 72(5): p. 1015-22.

35. Bakewell, D.J. and H. Morgan, Dielectrophoresis of DNA: Time- and frequencydependent collections on microelectrodes. leee Transactions on Nanobioscience, 2006. 5(1): p. 1-8.

36. Righetti, P.G. and A. Bossi, Isoelectric focusing of proteins and peptides in gel slabs and in capillaries. Analytica Chimica Acta, 1998. 372(1-2): p. 1-19.

37. Caglio, S. and P.G. Righetti, On the pH dependence of polymerization efficiency, as investigated by capillary zone electrophoresis. ELECTROPHORESIS, 1993. 14(1): p. 554-558.

38. Righetti, P.G., Chapter 5 Applications of IEF, in Laboratory Techniques in Biochemistry and Molecular Biology, T.S. Work and E. Work, Editors. 1976, Elsevier. p. 527-572.

39. Glenner, G.G. and C.W. Wong, Alzheimer's disease: Initial report of the purification and characterization of a novel cerebrovascular amyloid protein. Biochemical and Biophysical Research Communications, 1984. 120(3): p. 885-890.

40. Selkoe, D.J., Cell biology of protein misfolding: The examples of Alzheimer's and Parkinson's diseases. Nat Cell Biol, 2004. 6(11): p. 1054-1061. 
41. Westermeier, R. and R. Marouga, Protein detection methods in proteomics research. Bioscience Reports, 2005. 25(1-2): p. 19-32.

42. Karlsson, R., A. Michaelsson, and L. Mattsson, Kinetic analysis of monoclonal antibody-antigen interactions with a new biosensor based analytical system. Journal of Immunological Methods, 1991. 145(1-2): p. 229-240.

43. Gale, B.K., K.D. Caldwell, and A.B. Frazier, A micromachined electrical field-flow fractionation (/spl mu/-EFFF) system. Biomedical Engineering, IEEE Transactions on, 1998. 45(12): p. 1459-1469.

44. Verzola, B., C. Gelfi, and P.G. Righetti, Quantitative studies on the adsorption of proteins to the bare silica wall in capillary electrophoresis: II. Effects of adsorbed, neutral polymers on quenching the interaction. Journal of Chromatography A, 2000. 874(2): p. 293-303.

45. Lee, H.Y., et al., Electrochemical hematocrit determination in a direct current microfluidic device. Electrophoresis, 2015. 36(7-8): p. 978-985.

46. Lee, H.Y., C. Barber, and A.R. Minerick, Improving electrokinetic microdevice stability by controlling electrolysis bubbles. Electrophoresis, 2014. 35(12-13): p. 1782-1789.

47. Amro, N.A., S. Xu, and G.-y. Liu, Patterning Surfaces Using Tip-Directed Displacement and Self-Assembly. Langmuir, 2000. 16(7): p. 3006-3009.

48. He, Q., et al., Graphene-based electronic sensors. Chemical Science, 2012. 3(6): p. 1764-1772.

49. Jaschke, M. and H.-J. Butt, Deposition of Organic Material by the Tip of a Scanning Force Microscope. Langmuir, 1995. 11(4): p. 1061-1064.

50. Martinez-Duarte, R., Microfabrication technologies in dielectrophoresis applications - a review. Electrophoresis, 2012: p. n/a-n/a.

51. $\mathrm{Xu}, \mathrm{S}$. and G.-y. Liu, Nanometer-Scale Fabrication by Simultaneous Nanoshaving and Molecular Self-Assembly. Langmuir, 1997. 13(2): p. 127-129.

52. Piner, R.D., et al., "Dip-Pen" Nanolithography. Science, 1999. 283(5402): p. 661663.

53. Berdichevsky, Y., et al., UV/ozone modification of poly(dimethylsiloxane) microfluidic channels. Sensors and Actuators B: Chemical, 2004. 97(2-3): p. 402408.

54. Oláh, A., H. Hillborg, and G.J. Vancso, Hydrophobic recovery of UV/ozone treated poly(dimethylsiloxane): adhesion studies by contact mechanics and mechanism of surface modification. Applied Surface Science, 2005. 239(3-4): p. 410-423.

55. Vig, J.R., UV/ozone cleaning of surfaces. Journal of Vacuum Science \& Technology A, 1985. 3(3): p. 1027-1034.

56. Bio-Rad. A Guide to Polyacrylamide Gel Electrophoresis and Detection. Available from: http://www.bio-rad.com/webroot/web/pdf/lsr/literature/Bulletin 6040.pdf.

57. Candiano, G., et al., Blue silver: A very sensitive colloidal Coomassie G-250 staining for proteome analysis. ELECTROPHORESIS, 2004. 25(9): p. 1327-1333.

58. Pirmoradian, M., et al., Membrane-Assisted Isoelectric Focusing Device As a Micropreparative Fractionator for Two-Dimensional Shotgun Proteomics. Analytical Chemistry, 2014. 86(12): p. 5728-5732.

59. Nelson, W.C. and C.-J.C. Kim, Droplet Actuation by Electrowetting-on-Dielectric (EWOD): A Review. Journal of Adhesion Science and Technology, 2012. 26(1217): p. 1747-1771.

60. Srinivasan, V., V.K. Pamula, and R.B. Fair, An integrated digital microfluidic labon-a-chip for clinical diagnostics on human physiological fluids. Lab on a Chip, 2004. 4(4): p. 310-315. 
61. Lou, Y., L.M. Lunardi, and J.F. Muth, Fabrication of Nanoshell Arrays Using Directed Assembly of Nanospheres. leee Sensors Journal, 2010. 10(3): p. 617620.

62. Vengasandra, S.G., et al., Microfluidic ultramicroscale deposition and patterning of quantum dots. Nanotechnology, 2005. 16(10): p. 2052-2055.

63. Horka, M., et al., Capillary isoelectric focusing with UV-induced fluorescence detection. Journal of Chromatography A, 2001. 916(1-2): p. 65-71.

64. Righetti, P.G., Determination of the isoelectric point of proteins by capillary isoelectric focusing. Journal of Chromatography A, 2004. 1037(1-2): p. 491-499.

65. Dolník, V., Capillary electrophoresis of proteins 2003-2005. ELECTROPHORESIS, 2006. 27(1): p. 126-141.

66. Chingin, K., et al., Separation of Polypeptides by Isoelectric Point Focusing in Electrospray-Friendly Solution Using a Multiple-Junction Capillary Fractionator. Analytical Chemistry, 2012. 84(15): p. 6856-6862.

67. Yang, K.S., et al., Free-flow isoelectric focusing microfluidic device with glass coating by sol-gel methods. Current Applied Physics, 2009. 9(2): p. E66-E70.

68. Shimura, K., Recent advances in IEF in capillary tubes and microchips. Electrophoresis, 2009. 30(1): p. 11-28.

69. Ishibashi, R., T. Kitamori, and K. Shimura, Two-step perpendicular free-solution isoelectric focusing in a microchamber array chip. Lab on a Chip, 2010. 10(19): p. 2628-2631.

70. Nano eNabler ${ }^{T M}$ system. Available from: http://www.bioforcenano.com/index.php?id=295.

71. $\mathrm{Xu}, \mathrm{J} . \mathrm{T}$., et al., Microfabricated quill-type surface patterning tools for the creation of biological micro/nano arrays. Biomedical Microdevices, 2004. 6(2): p. 117-123.

72. Xu, J.T., et al., Microfabricated "Biomolecular Ink Cartridges" - Surface patterning tools (SPTS) for the printing of multiplexed biomolecular arrays. Sensors and Actuators B-Chemical, 2006. 113(2): p. 1034-1041.

73. Onoue, M. and H. Ushijima, Fabrication for metal photomask pattern by using fountain-pen nanolithography. Microelectronic Engineering, 2010. 87(5-8): p. 910913.

74. Baba, A., et al., Micro/nanopatterning of single-walled carbon nanotube-organic semiconductor composites. Nanotechnology, 2009. 20(8).

75. Cady, N.C., et al. Biologically self-assembled memristive circuit elements. in Circuits and Systems (ISCAS), Proceedings of 2010 IEEE International Symposium on. 2010.

76. Fahrenkopf, N.M., et al., Direct attachment of DNA to semiconducting surfaces for biosensor applications. J Biotechnol, 2010. 150(3): p. 312-4.

77. Fereol, S., et al., Micropatterned ECM substrates reveal complementary contribution of low and high affinity ligands to neurite outgrowth. Cytoskeleton (Hoboken), 2011. 68(7): p. 373-88.

78. Huang, N.F., et al., A matrix micropatterning platform for cell localization and stem cell fate determination. Acta Biomater, 2010. 6(12): p. 4614-21.

79. Islam, M.S., et al., High sensitive detection of C-reactive protein by total internal reflection fluorescence microscopy on rapidly making nanoarray protein chip. Talanta, 2010. 81(4-5): p. 1402-1408.

80. Korostynska, O., et al., Polymer Based Micro Sensors Arrays for Ph and Glucose Monitoring. Key Engineering Materials, 2010. 437: p. 354-358.

81. Lynch, M., et al., Functional protein nanoarrays for biomarker profiling. Proteomics, 2004. 4(6): p. 1695-1702. 
82. Mei, Y., et al., Cell-Compatible, Multicomponent Protein Arrays with Subcellular Feature Resolution. Small, 2008. 4(10): p. 1600-1604.

83. Neto, A.I., et al., High-throughput evaluation of interactions between biomaterials, proteins and cells using patterned superhydrophobic substrates. Soft Matter, 2011. 7(9): p. 4147.

84. Onoue, M., M. Fujita, and H. Ushijima, Wide Area Patterning of Organic Silane Molecules by Fountain-Pen Nanolithography. Molecular Crystals and Liquid Crystals, 2009. 505: p. 356-361.

85. Panda, S. and S. Pyarajan, Lab-on-Chip Devices for Protein Analysis, in Encyclopedia of Microfluidics and Nanofluidics, D. Li, Editor. 2008, Springer US. p. 940-947.

86. Phizicky, E., et al., Protein analysis on a proteomic scale. Nature, 2003. 422(6928): p. 208-215.

87. Kohlheyer, D., et al., Miniaturizing free-flow electrophoresis - a critical review. Electrophoresis, 2008. 29(5): p. 977-993.

88. Sommer, G.J. and A.V. Hatch, IEF in microfluidic devices. Electrophoresis, 2009. 30(5): p. 742-757.

89. Dorfman, K.D., DNA electrophoresis in microfabricated devices. Reviews of Modern Physics, 2010. 82(4): p. 2903-2947.

90. Slais, K., RESOLUTION OF AMPHOLYTES FOCUSED IN A NATURAL PH GRADIENT MOVING IN A TAPERED CAPILLARY. Journal of Microcolumn Separations, 1995. 7(2): p. 127-135.

91. $\mathrm{Xu}, \mathrm{Z}$., et al., Investigation of the $\mathrm{pH}$ gradient formation and cathodic drift in microchip isoelectric focusing with imaged UV detection. ELECTROPHORESIS, 2010. 31(21): p. 3558-3565.

92. Jezierski, S., D. Belder, and S. Nagl, Microfluidic free-flow electrophoresis chips with an integrated fluorescent sensor layer for real time $\mathrm{pH}$ imaging in isoelectric focusing. Chemical Communications, 2013. 49(9): p. 904-906.

93. Startsev, M.A., et al., Nanochannel $\mathrm{pH}$ Gradient Electrofocusing of Proteins. Analytical Chemistry, 2013. 85(15): p. 7133-7138.

94. Kataoka, M., et al., Reagent-release capillary array-isoelectric focusing device as a rapid screening device for IEF condition optimization. Lab on a Chip, 2010. 10(24): p. 3341-3347.

95. Ou, J.J., et al., Integration of dialysis membranes into a poly(dimethylsiloxane) microfluidic chip for isoelectric focusing of proteins using whole-channel imaging detection. Analytical Chemistry, 2008. 80(19): p. 7401-7407.

96. Poitevin, M., G. Peltre, and S. Descroix, Use of quasi-isoelectric buffers as anolyte and catholyte to improve capillary isoelectric focusing performances. Electrophoresis, 2008. 29(8): p. 1687-1693.

97. Hagedorn, R., et al., Electrophoresis in gel channels. Electrophoresis, 2005. 26(13): p. 2495-2502.

98. Cheng, J.H., et al., High-performance protein separation by ion exchange membrane partitioned free-flow isoelectric focusing system. Chemical Engineering Science, 2008. 63(8): p. 2241-2251.

99. Shen, Y.F. and R.D. Smith, Proteomics based on high-efficiency capillary separations. Electrophoresis, 2002. 23(18): p. 3106-3124.

100. Dolnik, V., Capillary electrophoresis of proteins 2003-2005. Electrophoresis, 2006. 27(1): p. 126-141. 
101. Canelle, L., et al., Automating proteome analysis: improvements in throughput, quality and accuracy of protein identification by peptide mass fingerprinting. Rapid Communications in Mass Spectrometry, 2004. 18(23): p. 2785-2794.

102. Svensson, H., Isoelectric fractionation, analysis, and characterisation of ampholytes in natural $\mathrm{pH}$ gra-dients. I. The differential equation of solute concentrations at a steady state and its solution for simple cases. Acta chem. scand, 1961. 15(2).

103. Cramer, R. and H. Svensson, Density gradient electrophoresis as a new tool in virology. Experientia, 1961. 17: p. 49-57.

104. McNaught, A.D. and A. Wilkinson, IUPAC. Compendium of Chemical Terminology, 2nd ed. (the "Gold Book"). 2 ed. 1997: Blackwell Scientific Publications.

105. Harris, D.C., Quantitative Chemical Analysis. 2010: W. H. Freeman.

106. Wren, S.A.C., Peak capacity in gradient ultra performance liquid chromatography (UPLC). Journal of Pharmaceutical and Biomedical Analysis, 2005. 38(2): p. 337343.

107. Rehman, F.N., et al., Immobilization of acrylamide-modified oligonucleotides by co-polymerization. Nucleic Acids Research, 1999. 27(2): p. 649-655.

108. Menter, P. Acrylamide Polymerization - A Practical Approach. 2000; Available from: http://www.bio-rad.com/webroot/web/pdf/lsr/literature/Bulletin 1156.pdf.

109. Kolin, A., ISOELECTRIC SPECTRA AND MOBILITY SPECTRA: A NEW APPROACH TO ELECTROPHORETIC SEPARATION. Proceedings of the National Academy of Sciences of the United States of America, 1955. 41(3): p. 101-10.

110. Nguyen, N.Y. and A. Chrambach, Nonisoelectric focusing in buffers. Analytical Biochemistry, 1976. 74(1): p. 145-153.

111. Basset, P., et al., Isoelectric focusing of human hemoglobin: its application to screening, to the characterization of 70 variants, and to the study of modified fractions of normal hemoglobins. Blood, 1978. 51(5): p. 971-982.

112. Drysdale, J.W., P. Righetti, and H.F. Bunn, The separation of human and animal hemoglobins by isoelectric focusing in polyacrylamide gel. Biochimica et Biophysica Acta (BBA) - Protein Structure, 1971. 229(1): p. 42-50.

113. Rowley, P.T., et al., High resolution analysis of hemoglobins: Polyacrylamide isoelectric focusing. Biochemical Medicine, 1972. 6(6): p. 553-560.

114. Lewis, U.J., E.V. Cheever, and B.K. Seavey, Purification of bovine growth hormone and prolactin by preparative electrophoresis. Analytical Biochemistry, 1968. 24(1): p. 162-175.

115. Mosher, R.A., W. Thormann, and M. Bier, Experimental and Theoretical Dynamics of Isoelectric-Focusing .2. Elucidation of the Impact of the Electrode Assembly. Journal of Chromatography, 1988. 436(2): p. 191-204.

116. Neuhoff, V., et al., Improved Staining of Proteins in Polyacrylamide Gels Including Isoelectric-Focusing Gels with Clear Background at Nanogram Sensitivity Using Coomassie Brilliant Blue G-250 and R-250. Electrophoresis, 1988. 9(6): p. 255262.

117. Righetti, P.G., E. Wenisch, and M. Faupel, PREPARATIVE PROTEINPURIFICATION IN A MULTI-COMPARTMENT ELECTROLYZER WITH IMMOBILINE MEMBRANES. Journal of Chromatography, 1989. 475: p. 293-309.

118. Deml, M., J. Pospichal, and J. Chmelik, Continuous Micropreparative Trapping in Carrier Ampholyte-Free Isoelectric-Focusing. Journal of Chromatography A, 1995. 709(1): p. 39-49. 
119. Klose, J. and U. Kobalz, 2-Dimensional Electrophoresis of Proteins - an Updated Protocol and Implications for a Functional-Analysis of the Genome. Electrophoresis, 1995. 16(6): p. 1034-1059.

120. Baker, J.E., Application of capillary thin layer isoelectric focusing in polyacrylamide gel to the study of alkaline proteinases in stored-product insects. Comparative Biochemistry and Physiology Part B: Comparative Biochemistry, 1982. 71(3): p. 501-506.

121. Hjerten, S. and M.D. Zhu, ADAPTATION OF THE EQUIPMENT FOR HIGHPERFORMANCE ELECTROPHORESIS TO ISOELECTRIC-FOCUSING. Journal of Chromatography, 1985. 346(OCT): p. 265-270.

122. Shen, Y., et al., High-Resolution Capillary Isoelectric Focusing of Complex Protein Mixtures from Lysates of Microorganisms. Analytical Chemistry, 1999. 71(23): p. 5348-5353.

123. Wu, J. and J. Pawliszyn, Universal detection for capillary isoelectric focusing without mobilization using concentration gradient imaging system. Analytical Chemistry, 1992. 64(2): p. 224-227.

124. Wu, J. and J. Pawliszyn, Dual Detection for Capillary Isoelectric Focusing with Refractive Index Gradient and Absorption Imaging Detectors. Analytical Chemistry, 1994. 66(6): p. 867-873.

125. Wu, J., et al., Capillary isoelectric focusing with whole column detection and a membrane sample preparation system. Analytica Chimica Acta, 1999. 383(1-2): p. 67-78.

126. Macounová, K., et al., Generation of Natural pH Gradients in Microfluidic Channels for Use in Isoelectric Focusing. Analytical Chemistry, 2000. 72(16): p. 3745-3751.

127. Griebel, A., et al., Integrated polymer chip for two-dimensional capillary gel electrophoresis. Lab on a Chip, 2004. 4(1): p. 18-23.

128. Albrecht, J.W. and K.F. Jensen, Micro free-flow IEF enhanced by active cooling and functionalized gels. Electrophoresis, 2006. 27(24): p. 4960-4969.

129. Dusa, F. and K. Slais, New solution IEF device for micropreparative separation of peptides and proteins. Electrophoresis, 2013. 34(11): p. 1519-25.

130. Wen, J., J.W. Albrecht, and K.F. Jensen, Microfluidic preparative free-flow isoelectric focusing in a triangular channel: System development and characterization. ELECTROPHORESIS, 2010. 31(10): p. 1606-1614.

131. Shim, J., P. Dutta, and C.F. Ivory, Dispersion of protein bands in a horseshoe microchannel during IEF. ELECTROPHORESIS, 2009. 30(5): p. 723-731.

132. Herr, A.E., et al., On-Chip Coupling of Isoelectric Focusing and Free Solution Electrophoresis for Multidimensional Separations. Analytical Chemistry, 2003. 75(5): p. 1180-1187.

133. Cui, H., et al., Multistage Isoelectric Focusing in a Polymeric Microfluidic Chip. Analytical Chemistry, 2005. 77(24): p. 7878-7886.

134. Zilberstein, G.V., E.M. Baskin, and S. Bukshpan, Parallel processing in the isoelectric focusing chip. ELECTROPHORESIS, 2003. 24(21): p. 3735-3744.

135. Kim, K.H., et al., Two dimensional (pl \&amp; $d s)$ separation of phosphorylated proteins by isoelectric focusing/asymmetrical flow field-flow fractionation: Application to prostatic cancer cell line. Journal of Proteomics, 2012. 75(8): p. 2297-2305.

136. Weiss, N.G., et al., Isoelectric Focusing in a Drop. Langmuir, 2011. 27(1): p. 494498.

137. Kohlheyer, D., et al., Microfluidic high-resolution free-flow isoelectric focusing. Analytical Chemistry, 2007. 79(21): p. 8190-8198. 
138. Walowski, B., W. Huttner, and H. Wackerbarth, Generation of a miniaturized freeflow electrophoresis chip based on a multi-lamination technique-isoelectric focusing of proteins and a single-stranded DNA fragment. Analytical and Bioanalytical Chemistry, 2011. 401(8): p. 2465-2471.

139. Tentori, A.M. and A.E. Herr, Performance implications of chemical mobilization after microchannel IEF. Electrophoresis, 2014. 35(10): p. 1453-1460.

140. Sommer, G.J., A.K. Singh, and A.V. Hatch, On-chip isoelectric focusing using photopolymerized immobilized pH gradients. Analytical Chemistry, 2008. 80(9): p. 3327-3333.

141. Kolin, A., Rapid electrophoresis in density gradients combined with $\mathrm{pH}$ and/or conductivity gradients. Methods of biochemical analysis, 1958. 6: p. 259-88.

142. Bjellqvist, B., et al., Isoelectric-Focusing in Immobilized Ph Gradients - Principle, Methodology and Some Applications. Journal of Biochemical and Biophysical Methods, 1982. 6(4): p. 317-339.

143. Zhu, M., R. Rodriguez, and T. Wehr, Optimizing separation parameters in capillary isoelectric focusing. Journal of Chromatography A, 1991. 559(1): p. 479-488.

144. Colyer, C.L., Unusual peaks and baseline shifts in capillary electrophoresis. J Capillary Electrophor, 1996. 3(3): p. 131-7.

145. Naydenov, C.L., A STUDY ON THE ROLE OF ELECTRODE SOLUTIONS IN THE FORMATION OF PH GRADIENTS IN POLYACRYLAMIDE GELS DURING ISOELECTRIC-FOCUSING. Dokladi Na Bolgarskata Akademiya Na Naukite, 1992. 45(5): p. 63-66.

146. Serva. Isoelectric Focusing, Regents and Equipments. 2015; Available from: http://www.serva.de/www root/documents/All\%20you\%20need\%20for\%20I EF\%20PAGE Intl 15-08-15.pdf.

147. Park, T.G., S. Cohen, and R. Langer, Poly(L-lactic acid)/Pluronic blends: characterization of phase separation behavior, degradation, and morphology and use as protein-releasing matrixes. Macromolecules, 1992. 25(1): p. 116-122.

148. Tsai, S.W., et al., Application of plasma-polymerized films for isoelectric focusing of proteins in a capillary electrophoresis chip. Analyst, 2003. 128(3): p. 237-244.

149. Gao, L. and S.R. Liu, Cross-linked polyacrylarnide coating for capillary isoelectric focusing. Analytical Chemistry, 2004. 76(24): p. 7179-7186.

150. Li, C., et al., Isoelectric focusing in cyclic olefin copolymer microfluidic channels coated by polyacrylamide using a UV photografting method. ELECTROPHORESIS, 2005. 26(9): p. 1800-1806.

151. Hellmich, W., et al., Improved native UV laser induced fluorescence detection for single cell analysis in poly(dimethylsiloxane) microfluidic devices. Journal of Chromatography A, 2006. 1130(2): p. 195-200.

152. Yao, B., et al., High-speed, whole-column fluorescence imaging detection for isoelectric focusing on a microchip using an organic light emitting diode as light source. Analytical Chemistry, 2006. 78(16): p. 5845-5850.

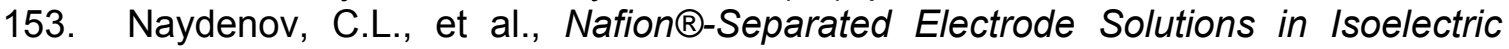
Focusing. Chromatographia, 2012. 75(1): p. 33-40.

154. Allen, R.C. and H.R. Maurer, Electropheresis and Isoelectric Focusing in Polyacrylamide Gel. 1974, Berlin: Walter de Gruyter.

155. Nguyen, N.Y., A.G. McCormick, and A. Chrambach, An anodic drift of $p H$ gradients in isoelectric focusing on polyacrylamide gel. Analytical Biochemistry, 1978. 88(1): p. 186-195.

156. Sasago, M., WHERE IS THE RESOLUTION LIMIT OF OPTICAL LITHOGRAPHY. 電気化学, 1987. 55(5): p. 350-354. 
157. Grenville, A., et al., MARKLE-DYSON OPTICS FOR 0.25-MU-M LITHOGRAPHY $A N D$ BEYOND. Journal of vacuum science \& technology. B, Microelectronics and nanometer structures processing, measurement and phenomena, 1991. 9(6): $p$. 3108-3112.

158. Okazaki, S., Resolution limits of optical lithography. Journal of Vacuum Science \& Technology B, 1991. 9(6): p. 2829-2833.

159. Stoyanov, A.V., et al., Conductivity properties of carrier ampholyte $\mathrm{pH}$ gradients in isoelectric focusing. ELECTROPHORESIS, 2005. 26(2): p. 473-479.

160. Rodriguez-Diaz, R., T. Wehr, and M.D. Zhu, Capillary isoelectric focusing. Electrophoresis, 1997. 18(12-13): p. 2134-2144.

161. Thormann, W., R.A. Mosher, and M. Bier, EXPERIMENTAL AND THEORETICAL DYNAMICS OF ISOELECTRIC-FOCUSING - ELUCIDATION OF A GENERAL SEPARATION MECHANISM. Journal of chromatography A, 1986. 351(1): p. 1729.

162. Mosher, R.A., W. Thormann, and M. Bier, EXPERIMENTAL AND THEORETICAL DYNAMICS OF ISOELECTRIC-FOCUSING .2. ELUCIDATION OF THE IMPACT OF THE ELECTRODE ASSEMBLY. Journal of chromatography A, 1988. 436(2): p. 191-204.

163. Mosher, R.A., et al., EXPERIMENTAL AND THEORETICAL DYNAMICS OF ISOELECTRIC-FOCUSING .3. TRANSIENT MULTI-PEAK APPROACH TO EQUILIBRIUM OF PROTEINS IN SIMPLE BUFFERS. Journal of chromatography A, 1989. 478(1): p. 39-49.

164. Mosher, R.A. and W. Thormann, Experimental and theoretical dynamics of isoelectric focusing: IV. Cathodic, anodic and symmetrical drifts of the $\mathrm{pH}$ gradient. ELECTROPHORESIS, 1990. 11(9): p. 717-723.

165. Finlayson, G.R. and A. Chrambach, Isoelectric focusing in polyacrylamide gel and its preparative application. Analytical Biochemistry, 1971. 40(2): p. 292-311.

166. Pirmoradian, M., J. Astorga-Wells, and R.A. Zubarev, Multijunction Capillary Isoelectric Focusing Device Combined with Online Membrane-Assisted Buffer Exchanger Enables Isoelectric Point Fractionation of Intact Human Plasma Proteins for Biomarker Discovery. Analytical Chemistry, 2015. 87(23): p. 1184011846.

167. Hempe, J.M. and R.D. Craver, Quantification of Hemoglobin-Variants by Capillary Isoelectric-Focusing. Clinical Chemistry, 1994. 40(12): p. 2288-2295.

168. Hofmann, O., et al., Adaptation of capillary isoelectric focusing to microchannels on a glass chip. Analytical Chemistry, 1999. 71(3): p. 678-686.

169. Tsai, S.-W., et al., Application of plasma-polymerized films for isoelectric focusing of proteins in a capillary electrophoresis chip. Analyst, 2003. 128(3): p. 237-244.

170. Macounova, K., C.R. Cabrera, and P. Yager, Concentration and separation of proteins in microfluidic channels on the basis of transverse IEF. Analytical Chemistry, 2001. 73(7): p. 1627-1633.

171. Lu, H., et al., A Microfabricated Device for Subcellular Organelle Sorting. Analytical Chemistry, 2004. 76(19): p. 5705-5712.

172. Garfin, D. and S. Ahuja, Handbook Of Isoelectric Focusing And Proteomics. Vol. 7. 2005, San Diego: Elsevier.

173. Gevaert, K. and J. Vandekerckhove, Protein identification methods in proteomics. ELECTROPHORESIS, 2000. 21(6): p. 1145-1154.

174. Jezierski, S., et al., Multistep liquid-phase lithography for fast prototyping of microfluidic free-flow-electrophoresis chips. Analytical and Bioanalytical Chemistry, 2011. 401(8): p. 2651-2656. 
175. Lin, F., et al., A novel microfluidic chip electrophoresis strategy for simultaneous, label-free, multi-protein detection based on a graphene energy transfer biosensor. Analyst, 2014. 139(11): p. 2890-2895.

176. Štěpánová, S. and V. Kašička, Analysis of proteins and peptides by electromigration methods in microchips. Journal of Separation Science, 2017. 40(1): p. 228-250.

177. Shameli, S.M. and C.L. Ren, Microfluidic Two-Dimensional Separation of Proteins Combining Temperature Gradient Focusing and Sodium Dodecyl SulfatePolyacrylamide Gel Electrophoresis. Analytical Chemistry, 2015. 87(7): p. 35933597.

178. Lin, F., et al., In situ photo-immobilised $\mathrm{pH}$ gradient isoelectric focusing and zone electrophoresis integrated two-dimensional microfluidic chip electrophoresis for protein separation. Microchimica Acta, 2015. 182(13): p. 2321-2328.

179. Xia, L., et al., On-chip protein isoelectric focusing using a photoimmobilized pH gradientt. Journal of Separation Science, 2014. 37(21): p. 3174-3180.

180. Gencoglu, A., et al., Quantification of $\mathrm{pH}$ gradients and implications in insulatorbased dielectrophoresis of biomolecules. Electrophoresis, 2011. 32(18): p. 24362447.

181. An, R., et al., Solution $\mathrm{pH}$ change in non-uniform alternating current electric fields at frequencies above the electrode charging frequency. Biomicrofluidics, 2014. 8(6): p. 13.

182. Nano eNabler ${ }^{T M}$ System - Benchtop Molecular Printer, B. Nanosciences, Editor.

183. Chapter 1 Theory and fundamental aspects of isoelectric focusing, in Laboratory Techniques in Biochemistry and Molecular Biology, R. Pier Giorgio, Editor. 1983, Elsevier. p. 1-86.

184. Das, C. and Z.H. Fan, Effects of separation length and voltage on isoelectric focusing in a plastic microfluidic device. ELECTROPHORESIS, 2006. 27(18): p. 3619-3626.

185. Rabilloud, T., Two-dimensional electrophoresis of basic proteins with equilibrium isoelectric focusing in carrier ampholyte-pH gradients. ELECTROPHORESIS, 1994. 15(1): p. 278-282.

186. Mao, Q. and J. Pawliszyn, Effect of salt concentration on separation patterns in static capillary isoelectric focusing with imaging detection. Journal of Chromatography B: Biomedical Sciences and Applications, 1999. 729(1-2): p. 355-359.

187. Poitevin, M., et al., Evaluation of microchip material and surface treatment options for IEF of allergenic milk proteins on microchips. ELECTROPHORESIS, 2009. 30(24): p. 4256-4263.

188. Hsu, W.-L., et al., Isoelectric Focusing in a Silica Nanofluidic Channel: Effects of Electromigration and Electroosmosis. Analytical Chemistry, 2014. 86(17): p. 87118718.

189. Xu, Y.-J., et al., Moving reaction boundary and isoelectric focusing: IV. Systemic study on Hjerten's pH gradient mobilization. Journal of Separation Science, 2009. 32(4): p. 585-596.

190. Shim, J., P. Dutta, and C.F. Ivory, Effects of ampholyte concentration on protein behavior in on-chip isoelectric focusing. ELECTROPHORESIS, 2008. 29(5): p. 1026-1035.

191. Tang, Q. and C.S. Lee, Effects of electroosmotic flow on zone mobilization in capillary isoelectric focusing. Journal of Chromatography A, 1997. 781(1-2): p. 113-118. 
192. Righetti, P.G., M. Chiari, and C. Gelfi, IMMOBILIZED PH GRADIENTS - EFFECT OF SALTS, ADDED CARRIER AMPHOLYTES AND VOLTAGE GRADIENTS ON PROTEIN-PATTERNS. Electrophoresis, 1988. 9(2): p. 65-73.

193. Strahler, J.R., et al., EFFECT OF SALT ON THE PERFORMANCE OF IMMOBILIZED PH GRADIENT ISOELECTRIC-FOCUSING GELS. Electrophoresis, 1988. 9(2): p. 74-80.

194. Skovgaard, I.M., K. Jensen, and I. Sondergaard, FROM IMAGE-PROCESSING TO CLASSIFICATION .3. MATCHING PATTERNS BY SHIFTING AND STRETCHING. Electrophoresis, 1995. 16(8): p. 1385-1389.

195. Poitevin, M., et al., Comparison of different capillary isoelectric focusing methodsuse of "narrow $\mathrm{pH}$ cuts" of carrier ampholytes as original tools to improve resolution. Journal of Chromatography A, 2007. 1155(2): p. 230-236.

196. Kremer, M., et al., Pore-Size Distributions of Cationic Polyacrylamide Hydrogels Varying in Initial Monomer Concentration and Cross-Linker/Monomer Ratio. Macromolecules, 1994. 27(11): p. 2965-2973.

197. Sayil, C. and O. Okay, Macroporous poly(N-isopropyl)acrylamide networks: formation conditions. Polymer, 2001. 42(18): p. 7639-7652.

198. Nam, K.H., et al., Continuous-flow fractionation of animal cells in microfluidic device using aqueous two-phase extraction. Biomedical Microdevices, 2005. 7(3): p. 189-195.

199. Nakano, A., et al., Immunoglobulin $G$ and bovine serum albumin streaming dielectrophoresis in a microfluidic device. Electrophoresis, 2011. 32(17): p. 23142322.

200. Stoyanov, A., IEF-based multidimensional applications in proteomics: Toward higher resolution. ELECTROPHORESIS, 2012. 33(22): p. 3281-3290.

201. Mack, S., et al., A systematic study in CIEF: defining and optimizing experimental parameters critical to method reproducibility and robustness. Electrophoresis, 2009. 30(23): p. 4049-4058.

202. Varenne, A. and S. Descroix, Recent strategies to improve resolution in capillary electrophoresis-A review. Analytica Chimica Acta, 2008. 628(1): p. 9-23.

203. Tan, W., et al., Miniaturized capillary isoelectric focusing in plastic microfluidic devices. Electrophoresis, 2002. 23(20): p. 3638-3645.

204. Páger, C., et al., Effect of electrolyte $\mathrm{pH}$ on CIEF with narrow $\mathrm{pH}$ range ampholytes. Electrophoresis, 2012. 33(22): p. 3269-3275.

205. Wu, D., et al., Multilayer poly(vinyl alcohol)-adsorbed coating on poly(dimethylsiloxane) microfluidic chips for biopolymer separation. ELECTROPHORESIS, 2005. 26(1): p. 211-218.

206. Yao, B., et al., High-Speed, Whole-Column Fluorescence Imaging Detection for Isoelectric Focusing on a Microchip Using an Organic Light Emitting Diode as Light Source. Analytical Chemistry, 2006. 78(16): p. 5845-5850.

207. Damaso, N., et al., F-108 polymer and capillary electrophoresis easily resolves complex environmental DNA mixtures and SNPs. ELECTROPHORESIS, 2014. 35(21-22): p. 3208-3211.

208. Wang, Z., C.F. Ivory, and A.R. Minerick, Surface Isoelectric Focusing (sIEF) with Carrier Ampholyte pH Gradient. Electrophoresis, 2016. Under revision.

209. Allard, E. and C. Larpent, Core-shell type dually fluorescent polymer nanoparticles for ratiometric $\mathrm{pH}$-sensing. Journal of Polymer Science Part A: Polymer Chemistry, 2008. 46(18): p. 6206-6213.

210. Meert, C., et al., Evaluation of $\mathrm{pl}$ marker sources for cIEF characterization of a therapeutic antibody. Chromatographia, 2007. 66(11-12): p. 963-968. 
211. Wang, M., et al., The intensification technologies to water electrolysis for hydrogen production - A review. Renewable and Sustainable Energy Reviews, 2014. 29: p. 573-588.

212. Xu, X., et al., Solution-Processed Zinc Oxide Thin-Film Transistors With a LowTemperature Polymer Passivation Layer. IEEE Electron Device Letters, 2012. 33(10): p. 1420-1422.

213. Ji, C.-H. and Y.-K. Kim, Electromagnetic Micromirror Array With Single-Crystal Silicon Mirror Plate and Aluminum Spring. Journal of Lightwave Technology, 2003. 21(3): p. 584.

214. Cain, J.L., Selective dry-etching of bi-layer passivation films. 1995, Google Patents.

215. Tang, K.C., et al., Evaluation of bonding between oxygen plasma treated polydimethyl siloxane and passivated silicon. Journal of Physics: Conference Series, 2006. 34(1): p. 155.

216. Schindler, M., et al., Novel post-process for the passivation of a CMOS biosensor. physica status solidi (RRL) - Rapid Research Letters, 2008. 2(1): p. 4-6.

217. Zou, Z., S. Lee, and C.H. Ahn, A polymer microfluidic chip with interdigitated electrodes arrays for simultaneous dielectrophoretic manipulation and impedimetric detection of microparticles. leee Sensors Journal, 2008. 8(5-6): p. 527-535.

218. Gusev, E.P., et al., Ultrathin high-K metal oxides on silicon: processing, characterization and integration issues. Microelectronic Engineering, 2001. 59(14): p. 341-349.

219. Aygun, G. and I. Yildiz, Interfacial and structural properties of sputtered HfO2 layers. Journal of Applied Physics, 2009. 106(1).

220. Franta, D., et al., Optical characterization of HfO2 thin films. Thin Solid Films, 2011. 519(18): p. 6085-6091.

221. Shim, J., J.A. Rivera, and R. Bashir, Electron beam induced local crystallization of HfO2 nanopores for biosensing applications. Nanoscale, 2013. 5(22): p. 1088710893.

222. Larkin, J., et al., Slow DNA Transport through Nanopores in Hafnium Oxide Membranes. ACS Nano, 2013. 7(11): p. 10121-10128.

223. Sim, S.P.C., et al., The shape of a step structure as a design aspect to control droplet generation in microfluidics. Journal of Micromechanics and Microengineering, 2010. 20(3): p. 035010.

224. Shen, S.-H., et al., An enhancement of high-k/oxide stacked dielectric structure for silicon-based multi-nanowire biosensor in cardiac troponin I detection. Sensors and Actuators B: Chemical, 2015. 218: p. 303-309.

225. Ghafar-Zadeh, E., M. Sawan, and D. Therriault, Novel direct-write CMOS-based laboratory-on-chip: Design, assembly and experimental results. Sensors and Actuators A: Physical, 2007. 134(1): p. 27-36.

226. Gabriel, N. and J. Talghader, Optical coatings in microscale channels by atomic layer deposition. Applied optics, 2010. 49(8): p. 1242-1248.

227. Moncada Hernandez, H., et al., Electrical and chemical characterizations of hafnium (IV) oxide films for biological Lab-on- a-Chip devices. Biosensors and Bioelectronics, 2017. Submitted.

228. Viefhues, M., et al., Physisorbed surface coatings for poly(dimethylsiloxane) and quartz microfluidic devices. Analytical and Bioanalytical Chemistry, 2011. 401(7): p. 2113-2122.

229. Peschle, C., et al., HEMOGLOBIN-SYNTHESIS IN INDIVIDUAL BURSTS FROM NORMAL ADULT-BLOOD - ALL BURSTS AND SUB-COLONIES SYNTHESIZE 
G-GAMMA-GLOBIN AND A-GAMMA-GLOBIN CHAINS. Blood, 1980. 56(2): p. 218-226.

230. Jenkins, M.A. and S. Ratnaike, Capillary isoelectric focusing of haemoglobin variants in the clinical laboratory. Clinica Chimica Acta, 1999. 289(1-2): p. 121-132.

231. Tang, S., et al., A method for routine analysis of recombinant immunoglobulins (rlgGs) by capillary isoelectric focusing (cIEF). Journal of Pharmaceutical and Biomedical Analysis, 1999. 19(3-4): p. 569-583.

232. He, X., A. Que, and J. Mo, Analysis of charge heterogeneities in mAbs using imaged CE. Electrophoresis, 2009. 30(5): p. 714-722.

233. Ruotolo, B.T., et al., Peak capacity of ion mobility mass spectrometry: the utility of varying drift gas polarizability for the separation of tryptic peptides. Journal of Mass Spectrometry, 2004. 39(4): p. 361-367.

234. Hofmann, O., et al., Adaptation of Capillary Isoelectric Focusing to Microchannels on a Glass Chip. Analytical Chemistry, 1999. 71(3): p. 678-686.

235. Nagai, Y., H.D. Klenk, and R. Rott, PROTEOLYTIC CLEAVAGE OF VIRAL GLYCOPROTEINS AND ITS SIGNIFICANCE FOR VIRULENCE OF NEWCASTLE-DISEASE VIRUS. Virology, 1976. 72(2): p. 494-508.

236. Kishi, K., T. Yasuda, and Y. Ikehara, Alpha-2-HS-Glycoprotein Polymorphism Detected in Human Urine by Isoelectric Focusing and Immunoblotting. Human Heredity, 1988. 38(4): p. 207-210.

237. Kremer, J.M.H., J. Wilting, and L.H.M. Janssen, DRUG-BINDING TO HUMAN ALPHA-1-ACID GLYCOPROTEIN IN HEALTH AND DISEASE. Pharmacological Reviews, 1988. 40(1): p. 1-47.

238. May, P.C., et al., Dynamics of gene expression for a hippocampal glycoprotein elevated in Alzheimer's disease and in response to experimental lesions in rat. Neuron, 1990. 5(6): p. 831-839.

239. Smit, J.J.M., et al., HOMOZYGOUS DISRUPTION OF THE MURINE MDR2 PGLYCOPROTEIN GENE LEADS TO A COMPLETE ABSENCE OF PHOSPHOLIPID FROM BILE AND TO LIVER-DISEASE. Cell, 1993. 75(3): p. 451462.

240. Lefkovits, J., E.F. Plow, and E.J. Topol, MECHANISMS OF DISEASE - PLATELET GLYCOPROTEIN IIB/IIIA RECEPTORS IN CARDIOVASCULAR MEDICINE. NeW England Journal of Medicine, 1995. 332(23): p. 1553-1559.

241. Rose, M.C. and J.A. Voynow, Respiratory tract mucin genes and mucin glycoproteins in health and disease. Physiological Reviews, 2006. 86(1): p. 245278.

242. Lacunza, I., et al., Comparison of $\alpha$-1-acid glycoprotein isoforms from healthy and cancer patients by capillary IEF. ELECTROPHORESIS, 2007. 28(23): p. 44474451.

243. Goochee, C.F., et al., The Oligosaccharides of Glycoproteins: Bioprocess Factors Affecting Oligosaccharide Structure and their Effect on Glycoprotein Properties. Nat Biotech, 1991. 9(12): p. 1347-1355.

244. Shopes, B., A genetically engineered human IgG mutant with enhanced cytolytic activity. The Journal of Immunology, 1992. 148(9): p. 2918-22.

245. Solá, R.J. and K. Griebenow, Glycosylation of Therapeutic Proteins. BioDrugs, 2010. 24(1): p. 9-21.

246. Dennis, J.W., M. Granovsky, and C.E. Warren, Protein glycosylation in development and disease. BioEssays, 1999. 21(5): p. 412-421.

247. Dwek, R.A., Glycobiology: Toward Understanding the Function of Sugars. Chemical Reviews, 1996. 96(2): p. 683-720. 
248. An, Y., et al., A new tool for monoclonal antibody analysis: Application of IdeS proteolysis in IgG domain-specific characterization. mAbs, 2014. 6(4): p. 879-893.

249. Leblanc, Y., et al., Glycation of polyclonal IgGs: Effect of sugar excipients during stability studies. European Journal of Pharmaceutics and Biopharmaceutics, 2016. 102: p. 185-190.

250. Tran, B.Q., et al., Glycosylation characterization of therapeutic mAbs by top-and middle-down mass spectrometry. Data in Brief, 2016. 6: p. 68-76.

251. Irungu, J., et al., Comparison of HPLC/ESI-FTICR MS versus MALDI-TOF/TOF MS for glycopeptide analysis of a highly glycosylated HIV envelope glycoprotein. Journal of the American Society for Mass Spectrometry, 2008. 19(8): p. 1209-1220.

252. Kim, Y.J., A. Freas, and C. Fenselau, Analysis of viral glycoproteins by MALDITOF mass spectrometry. Analytical Chemistry, 2001. 73(7): p. 1544-1548.

253. Karas, M., et al., MATRIX DEPENDENCE OF METASTABLE FRAGMENTATION OF GLYCOPROTEINS IN MALDI TOF MASS-SPECTROMETRY. Analytical Chemistry, 1995. 67(3): p. 675-679.

254. Manabe, T., A. Iwasaki, and H. Miyamoto, Separation of human plasma/serum proteins by capillary isoelectric focusing in the absence of denaturing agents. ELECTROPHORESIS, 1997. 18(7): p. 1159-1165.

255. Mao, Q. and J. Pawliszyn, Capillary isoelectric focusing with whole column imaging detection for analysis of proteins and peptides. Journal of Biochemical and Biophysical Methods, 1999. 39(1-2): p. 93-110.

256. Zarabadi, A.S. and J. Pawliszyn, Accurate Determination of the Diffusion Coefficient of Proteins by Fourier Analysis with Whole Column Imaging Detection. Analytical Chemistry, 2015. 87(4): p. 2100-2106.

257. Chen, G., S. Ha, and R. Rustandi, Characterization of Glycoprotein Biopharmaceutical Products by Caliper LC90 CE-SDS Gel Technology, in Glycosylation Engineering of Biopharmaceuticals, A. Beck, Editor. 2013, Humana Press. p. 199-209.

258. Hille, J.M., A.L. Freed, and H. Wätzig, Possibilities to improve automation, speed and precision of proteome analysis: A comparison of two-dimensional electrophoresis and alternatives. ELECTROPHORESIS, 2001. 22(19): p. 40354052.

259. Storms, H.F., et al., Capillary isoelectric focusing-mass spectrometry for shotgun approach in proteomics. ELECTROPHORESIS, 2004. 25(20): p. 3461-3467.

260. Silvertand, L.H.H., et al., Development and characterization of cIEF-MALDI-TOF MS for protein analysis. ELECTROPHORESIS, 2009. 30(10): p. 1828-1835.

261. Foret, F., et al., Analysis of protein fractions by micropreparative capillary isoelectric focusing and matrix-assisted laser desorption time-of-flight mass spectrometry. Journal of Chromatography A, 1995. 716(1): p. 157-166.

262. Yang, S., et al., Glycomic Analysis by Glycoprotein Immobilization for Glycan Extraction and Liquid Chromatography on Microfluidic Chip. Analytical Chemistry, 2013. 85(21): p. 10117-10125.

263. Chen, Y., L. Zhang, and G. Chen, Fabrication, modification, and application of poly(methyl methacrylate) microfluidic chips. ELECTROPHORESIS, 2008. 29(9): p. $1801-1814$.

264. Wang, Z. and A.R. Minerick, Surface Isoelectric Focusing (sIEF) for Hemoglobin Variants Separation with Narrow Range pH gradient Electrophoresis, 2017. ready to submit. 
265. Kinoshita, M., et al., Quality assurance of monoclonal antibody pharmaceuticals based on their charge variants using microchip isoelectric focusing method. Journal of Chromatography A, 2013. 1309: p. 76-83.

266. Leonard, K.M. and A.R. Minerick, Explorations of ABO-Rh antigen expressions on erythrocyte dielectrophoresis: Changes in cross-over frequency. Electrophoresis, 2011. 32(18): p. 2512-2522.

267. Liu, H., et al., Ranking the Susceptibility of Disulfide Bonds in Human IgG1 Antibodies by Reduction, Differential Alkylation, and LC-MS Analysis. Analytical Chemistry, 2010. 82(12): p. 5219-5226.

268. Gangopadhyay, A., A.T. Petrick, and P. Thomas, Modification of antibody isoelectric point affects biodistribution of 111-indium-labeled antibody. Nuclear Medicine and Biology, 1996. 23(3): p. 257-261.

269. Zhou, Q., et al., $N$-linked oligosaccharide analysis of glycoprotein bands from isoelectric focusing gels. Analytical Biochemistry, 2004. 335(1): p. 10-16.

270. Sosic, Z., et al., Application of imaging capillary IEF for characterization and quantitative analysis of recombinant protein charge heterogeneity. ELECTROPHORESIS, 2008. 29(21): p. 4368-4376.

271. Weiner, G.J., Building better monoclonal antibody-based therapeutics. Nat Rev Cancer, 2015. 15(6): p. 361-370.

272. Winstead, C.R., et al., Antigen-Induced Somatic Diversification of Rabbit IgH Genes: Gene Conversion and Point Mutation. The Journal of Immunology, 1999. 162(11): p. 6602-6612.

273. Wang, W., et al., Measurement of electroosmotic flow in capillary and microchip electrophoresis. Journal of Chromatography A, 2007. 1170(1-2): p. 1-8.

274. Lowe, D.G., Distinctive Image Features from Scale-Invariant Keypoints. International Journal of Computer Vision, 2004. 60(2): p. 91-110. 